\title{
Perpendicular magnetic anisotropy at transition metal/oxide interfaces and applications
}

\author{
B. Dieny ${ }^{*}$ and M. Chshiev ${ }^{\dagger}$ \\ Univ. Grenoble Alpes, INAC-SPINTEC, F-38000 Grenoble, France, \\ CEA, INAC-SPINTEC, F-38000 Grenoble, France, \\ and CNRS, SPINTEC, F-38000 Grenoble, France
}

(published 28 June 2017)

\begin{abstract}
Spin electronics is a rapidly expanding field stimulated by a strong synergy between breakthrough basic research discoveries and industrial applications in the fields of magnetic recording, magnetic field sensors, nonvolatile memories [magnetic random access memories (MRAM) and especially spin-transfer-torque MRAM (STT-MRAM)]. In addition to the discovery of several physical phenomena (giant magnetoresistance, tunnel magnetoresistance, spin-transfer torque, spin-orbit torque, spin Hall effect, spin Seebeck effect, etc.), outstanding progress has been made on the growth and nanopatterning of magnetic multilayered films and nanostructures in which these phenomena are observed. Magnetic anisotropy is usually observed in materials that have large spin-orbit interactions. However, in 2002 perpendicular magnetic anisotropy (PMA) was discovered to exist at magnetic metal/oxide interfaces [for instance $\mathrm{Co}(\mathrm{Fe})$ /alumina]. Surprisingly, this PMA is observed in systems where spin-orbit interactions are quite weak, but its amplitude is remarkably large-comparable to that measured at $\mathrm{Co} / \mathrm{Pt}$ interfaces, a reference for large interfacial anisotropy (anisotropy $\sim 1.4 \mathrm{erg} / \mathrm{cm}^{2}=1.4 \mathrm{~mJ} / \mathrm{m}^{2}$ ). Actually, this PMA was found to be very common at magnetic metal/oxide interfaces since it has been observed with a large variety of amorphous or crystalline oxides, including $\mathrm{AlOx}, \mathrm{MgO}, \mathrm{TaOx}, \mathrm{HfOx}$, etc. This PMA is thought to be the result of electronic hybridization between the oxygen and the magnetic transition metal orbit across the interface, a hypothesis supported by ab initio calculations. Interest in this phenomenon was sparked in 2010 when it was demonstrated that the PMA at magnetic transition metal/oxide interfaces could be used to build out-of-plane magnetized magnetic tunnel junctions for STT-MRAM cells. In these systems, the PMA at the $\mathrm{CoFeB} / \mathrm{MgO}$ interface can be used to simultaneously obtain good memory retention, thanks to the large PMA amplitude, and a low write current, thanks to a relatively weak Gilbert damping. These two requirements for memories tend to be difficult to reconcile since they rely on the same spin-orbit coupling. PMA-based approaches have now become ubiquitous in the designs for perpendicular STT-MRAM, and major microelectronics companies are actively working on their development with the first goal of addressing embedded FLASH and static random access memory-type of applications. Scalability of STT-MRAM devices based on this interfacial PMA is expected to soon exceed the 20 -nm nodes. Several very active new fields of research also rely on interfacial PMA at magnetic metal/oxide interfaces, including spin-orbit torques associated with Rashba or spin Hall effects, record high speed domain wall propagation in buffer/magnetic metal/oxide-based magnetic wires, and voltage-based control of anisotropy. This review deals with PMA at magnetic metal/oxide interfaces from its discovery, by examining the diversity of systems in which it has been observed and the physicochemical methods through which the key roles played by the electronic hybridization at the metal/oxide interface were elucidated. The physical origins of the phenomenon are also covered and how these are supported by $a b$ initio calculations is dealt with. Finally, some examples of applications of this interfacial PMA in STT-MRAM are listed along with the various emerging research topics taking advantage of this PMA.
\end{abstract}

DOI: 10.1103/RevModPhys.89.025008

\section{CONTENTS}

I. Introduction

II. PMA at Magnetic Transition Metal/Oxide Interfaces:

A Historical Perspective

A. First observation with AlOx formed by rf oxidation and natural oxidation

\footnotetext{
*bernard.dieny@cea.fr

†mair.chshiev@cea.fr
}

B. A general phenomenon at magnetic metal/oxide interface:

C. Correlation between PMA and electron-specular reflection in specular spin valves

5 D. Physicochemical characterization (XAS, XPS) of the metal/oxide interface and correlation with anisotropy

E. Influence of annealing

F. Correlation between PMA and tunnel magnetoresistance in $\mathrm{MgO}$-based MTJ

G. First observations of PMA at $\mathrm{Ta} / \mathrm{CoFeB} / \mathrm{MgO}$ interfaces 
H. Interfacial PMA in epitaxial Fe/MgO-based systems 14

I. Gilbert damping measurements in

$\mathrm{CoFeB} / \mathrm{MgO}$-based MTJ

III. Physical Origin of the Interfacial PMA

A. Qualitative description of anisotropy based on electronic hybridization

B. Ab initio calculations, comparison with experimental data

IV. STT-MRAM Based on Interfacial $\mathrm{CoFeB} / \mathrm{MgO}$ PMA

A. STT-MRAM: A subclass of MRAM

B. In-plane magnetized STT-MRAM with reduced demagnetizing field

C. Perpendicular STT-MRAM

D. Benefit of exploiting the interfacial PMA at $\mathrm{CoFeB} / \mathrm{MgO}$ interfaces when tackling the large anisotropy or low critical current dilemma

E. Influence of various parameters on the PMA measured at $\mathrm{CoFeB} / \mathrm{MgO}$ interfaces

1. Influence of buffer and capping layers

2. Influence of the presence of $\mathrm{Mg}$ between $\mathrm{MgO}$ and $\mathrm{CoFeB}$

3. Influence of $\mathrm{CoFeB}$ composition on PMA amplitude and thermal variation

4. Influence of annealing

a. Controlling the diffusion of atomic species

b. Influence of irradiation with light ions

F. Status of STT-MRAM development

V. Voltage Control of the Anisotropy and Applications

in Low-power Magnetic Memories

VI. Other Emerging Phenomena in Structures Based on Magnetic

Metal/Oxide Interfacial PMA

VII. Conclusion

VIII. Acronyms

Acknowledgments

References

\section{INTRODUCTION}

The properties of magnetic thin films and multilayers first triggered interest more than 60 years ago (Néel, 1954). Since then, a wealth of new phenomena have been discovered in these artificially made material systems. These phenomena are of interest not only from a fundamental point of view but also because they could have applications in various areas including, but not limited to, magnetic storage [hard disk drives (HDD) (Thompson and Best, 2000; Chappert, Fert, and Van Dau, 2007)], solid-state magnetic memories [magnetic random access memories (MRAM) (Huai, 2008; Khvalkovskiy et al., 2013)], hybrid complementary metal oxide semiconductor (CMOS) or magnetic logic (Dieny et al., 2010; Kawahara, 2011), rf components (Rippard et al., 2005; Dussaux et al., 2010; Choi et al., 2014; Miwa et al., 2014), magnetic field sensors (Reig, Cardoso de Freitas, and Mukhopadhyay, 2014), and biotechnology (Tartaj et al., 2005). All this research and development (R\&D) was made possible thanks to the tremendous technological progress achieved in techniques for deposition of magnetic thin films, in tools for their nanopatterning, in structural and chemical characterization techniques, and in the means to measure their physical properties. Among the remarkable phenomena and devices discovered and developed over the years, the following are of particular interest: the phenomenon of exchange bias at ferromagnetic/antiferromagnetic interfaces (Meiklejohn, 1962; Nogues and Schuller, 1999); the observation of perpendicular magnetic anisotropy (PMA) in thin films and enhanced magneto-optical Kerr rotation (Carcia, Meinhaldt, and Suna, 1985; Den Broeder et al., 1987; Den Broeder, Hoving, and Bloemen, 1991); giant magnetoresistance (GMR) (Baibich et al., 1988; Binasch et al., 1989), for which A. Fert and P. Grünberg were awarded the 2007 Physics Nobel Prize; low-field GMR spin valves (Dieny et al., 1991), which were made commercially available in HDD by IBM in 1998; the oscillatory exchangelike coupling between ferromagnetic (FM) layers separated by a nonmagnetic layer (Parkin, More, and Roche, 1990); tunnel magnetoresistance (TMR) at room temperature (Miyazaki and Tezuka, 1995; Moodera et al., 1995); the fact that it should be possible to manipulate the magnetization of magnetic nanostructures by spin-transfer torque (Berger, 1996; Slonczewski, 1996), and its first applications in excitation of spin waves (Tsoi et al., 1998) or magnetization switching (Wegrowe et al., 1999; Katine et al., 2000); the prediction and observation of giant tunnel magnetoresistance in $\mathrm{MgO}$-based crystalline magnetic tunnel junctions (MTJ) (Butler et al., 2001; Mathon and Umerski, 2001; Parkin et al., 2004; Yuasa et al., 2004); the strong interplay between charge-spinheat currents was harnessed and gave rise to the field of spin caloritronics (Saitoh et al., 2006; Bauer, Saitoh, and Van Wees, 2012); the prediction that spin angular momentum can be transferred from the lattice to the local magnetization by a current flow resulting in spin-orbit torques (SOT) (Manchon and Zhang, 2009); and the first observations of SOT-induced magnetization switching due to Rashba or spin Hall effects (SHE) (Miron et al., 2010; Miron, Garello et al., 2011; Liu, Lee et al., 2012; Liu, Pai et al., 2012).

Since their introduction in 1954, the development of magnetic HDD has been a strong driving force in micromagnetism, nanomagnetism, and spin electronics. The HDD industry has followed a road map very similar to the well-known Moore's law in the semiconductor industry, where the number of components per unit area doubles every two years (i.e., $41 \%$ increase per year). Thus in HDDs, the areal density of information stored has increased at an average rate of $60 \%$ per year, representing more than 8 orders of magnitude over 50 years.

Information can be stored very efficiently by controlling the orientation of small magnetic domains (bits). For binary storage, the magnetic media onto which information is written is designed to have two stable states at remanence. This is achieved with uniaxial magnetic anisotropy, which provides a preferred orientation for the magnetization. The magnetization therefore has two possible directions along the anisotropy axis. This uniaxial anisotropy is generally characterized by an anisotropy energy per unit volume $K_{u}$, so that the anisotropy energy varies depending on the orientation of the magnetization $\vec{M}$ with respect to the easy axis of magnetization ( $\hat{n}$ unit vector along this axis) as

$$
E=-K_{u}(\vec{M} \cdot \hat{n})^{2}
$$

To allow the increase in areal density in granular HDD media consisting of CoCrPt alloy grains, the size had to be steadily decreased as the storage density increased. 
At today's densities, around $700 \mathrm{Gbit} / \mathrm{in}^{2}$, the grain size is approximately $7 \mathrm{~nm}$. The number of grains per bit has also decreased over time, from more than 100 initially to about 10 nowadays. Writing the information requires the magnetization of the grains to switch from one stable state to the other by overcoming an energy barrier $\Delta E$, which separates the two stable states. The energy required is given by

$$
\Delta E=K_{u} V,
$$

where $K_{u}$ is the anisotropy per unit volume of the storage layer and $V$ its volume, i.e., the grain size. $\Delta E$ determines the stability of the written information. If the barrier is not high enough, the magnetization of a grain may accidently switch to the opposite direction due to thermal fluctuations $\left(k_{B} T\right)$. This will result in degradation of the information stored. The typical time for switching by overcoming the energy barrier is given by an Arrhenius law

$$
\tau=\tau_{0} \exp \left(\frac{\Delta E}{k_{B} T}\right)
$$

where $\tau_{0}$ is a characteristic attempt time of the order of $1 \mathrm{~ns}$. For the information to remain stable throughout a specified retention time $t_{\text {retention }}$ (typically 10 years in HDD), the energy barrier must comply with

$$
\Delta E>k_{B} T \log \left(\frac{t_{\text {retention }}}{\tau_{0}}\right),
$$

i.e.,

$$
\Delta E=K_{u} V>45 k_{B} T
$$

for a 10-year retention. Over the years, as the storage density increased, the volume of grains had to be decreased. Therefore, to fulfill the condition $K_{u} V>45 k_{B} T$, the anisotropy had to be increased correspondingly. This was achieved by improving the texture of the hcp-CoCr-based alloys, reinforcing the spin-orbit coupling by Pt doping and, most importantly, by switching from in-plane to out-of-plane anisotropy to reduce the negative impact that demagnetizing energy has on the effective anisotropy as the bit size decreased (Piramanayagama, 2007). Today, the areal density in HDD is close to $1 \mathrm{Tbit} / \mathrm{in}^{2}$, but conventional recording technology, based on perpendicular media and classical write heads, is approaching the limit associated with the writability of the media. Indeed, the anisotropy of the media has been increased so much to fulfill the magnetic stability criterion that the field produced by the write head, which is determined by the magnetization of the head pole piece, is no longer sufficient to overcome the $K_{u} V$ barrier to switch magnetization. To circumvent this issue, new approaches are being developed using graded media (Suess et al., 2005) or using recordings assisted either by microwaves [microwave-assisted magnetic recording (MAMR) (Zhu, Zhu, and Tang, 2008)] or by temporarily heating the media using a plasmonic antenna [heat assisted magnetic recording (HAMR) (Stipe et al., 2010)].
Magnetism can also be used to store data in solid-state memories, known as MRAM. These memories, unlike HDD, have no moving parts. In 1995 TMR was observed in MTJ at room temperature (RT) (Miyazaki and Tezuka, 1995; Moodera et al., 1995), and subsequently it was proposed that MTJs could serve as memory elements. An MTJ consists of two magnetic layers separated by an insulating tunnel barrier. The resistance of the barrier depends on the relative orientation of the magnetization in the two magnetic layers. In a parallel magnetic configuration, electrons can easily tunnel through the barrier, therefore the MTJ is in a low resistance state. With antiparallel alignment, electron tunneling is hampered, producing a high resistance state in the MTJ. MTJs are usually designed so that one of the two magnetic layers, the reference layer, has a fixed magnetization. The magnetization of the second layer, the storage layer, can be modified either by pulses of magnetic field or by using the spin-transfer-torque (STT) phenomenon. For further details, see Dieny et al. (2010) and Sec. IV. In MRAM, memory retention is once again directly related to the barrier height $K_{u} V$. As in magnetic recording, if the memory cell size is reduced to increase the storage capacity, the anisotropy also has to be increased to meet the criterion for thermal stability. This stability is measured for MRAM based on the failure rate in standby. For an $N$-bit memory, the failure rate can be calculated as a function of time by writing the probability that at least one failure will occur during a period $t$ as 1 minus the probability that no such failure will occur in any of the $N$ bits during the same period. This yields the following expression:

$F(t)=1-\exp (-N t / \tau)=1-\exp \left[\frac{-N t}{\tau_{0}} \exp \left(-\frac{\Delta E}{k_{B} T}\right)\right]$

For a given retention time $t=t_{\text {retention }}$ and a given chip capacity, this relationship sets a minimum value for the thermal stability ratio $\Delta E / k_{B} T$ and therefore for $K_{u} V$.

These considerations show that the anisotropy of a magnetic material plays a key role in important applications of magnetism, particularly those related to memory and storage.

Magnetic anisotropy in thin films and multilayers has been studied for more than 60 years (Néel, 1954). It is now known that magnetic anisotropy can have bulk and interfacial contributions stemming from a common origin: the spin-orbit interaction (SOI). This interaction links the orbital motion of the electrons, which is determined by the lattice arrangement, to their spin, which constitutes the main contribution to magnetization in magnetic transition metals. Therefore the spin-orbit interaction induces pairing between the magnetization and the crystallographic lattice (Bethe, 1929; Bloch and Gentile, 1931; Ballhausen, 1962; Mattheiss, 1972). This interaction is associated with a number of important phenomena in magnetism such as magnetic anisotropy, the relaxation of magnetic excited states toward the lattice, as characterized by Gilbert damping $\alpha$, or magnetostriction phenomena. Large anisotropies are commonly observed in materials which have large spin-orbit interactions, such as heavy elements (Pt, $\mathrm{Au}$, $\mathrm{Ta}, \mathrm{W}, \mathrm{Bi}$, rare earths with nonzero orbital momentum, etc.). Indeed, conventionally the spin-orbit-coupling constant in the simplest model of atom description with Bohr radius scales as $Z^{4}$. More precisely, the spin-orbit-coupling energy is 
proportional to $\alpha^{2} Z^{4}$, where $\alpha$ is the fine structure constant. However, for $d$ elements this is oversimplified and overestimated since it neglects screening effects due to the outer electrons. More detailed theoretical investigations indicate that the spin-orbit-coupling energy may scale as $\alpha^{2} Z^{2}$, in particular, in transition metals (Khomski, 2014).

Characteristic examples of multilayers considered to have large anisotropy associated with a large spin orbit are $\mathrm{Co} / \mathrm{Pd}$ and Co/Pt multilayers (Johnson et al., 1995). In bulk form, examples of systems with large anisotropy include hexagonal $\mathrm{CoPtCr}$ alloys which are used in media for HDD and FePt $L_{10^{-}}$ ordered alloys with tetragonal distortion (Gehanno et al., 1997). In these two examples, the reduced crystal symmetry combined with the large spin orbit of Pt results in strong magnetocrystalline anisotropy (MCA). In multilayers, the anisotropy can be of various origins. As early as 1954, Néel (1954) proposed a pair model according to which the broken symmetry at interfaces can generate anisotropy. Another contribution can arise from the crystallographic mismatch between neighboring elements generating strains in the layers which produces anisotropy due to magnetostriction effects (Johnson et al., 1995). Electron hybridization across the interface may also increase the levels of degeneracy between orbitals, generating interfacial anisotropy (Daalderop, Kelly, and Schuurmans, 1994a, 1994b).

In addition to these contributions, which are related to the material itself, for magnetostatic reasons the shape of the magnetic element also contributes to the anisotropy measured. In infinite thin films, in the absence of other forms of anisotropy, magnetization will spontaneously lie in the plane of the film (easy-plane anisotropy). Energy

$$
E_{\text {demag }}=-\frac{\mu_{0}}{2} M_{s}^{2},
$$

where $M_{s}$ is the spontaneous magnetization in SI units, must be supplied to bring it out of plane. If the film is patterned in small elements, the precise demagnetizing coefficients corresponding to the sample geometry must be taken into account (Beleggia et al., 2005). For a thin magnetic film placed between two interfaces, the anisotropy is usually expressed as an effective anisotropy per unit volume, thus,

$$
K_{\mathrm{eff}}=\left(K_{v}-\frac{\mu_{0}}{2} M_{s}^{2}\right)+\frac{K_{s}}{t},
$$

where $K_{v}$ accounts for all the material bulk anisotropy contributions, $-\left(\mu_{0} / 2\right) M_{s}^{2}$ represents the demagnetizing energy, and $K_{s}$ combines all the interfacial contributions from the two interfaces. Experimentally, the product $K_{\text {eff }} t$ can be plotted against $t$ to determine the various contributions. The slope of this curve gives the net bulk anisotropy, whereas the intercept with vertical axis yields the net interfacial anisotropy.

This review deals with a particular form of interfacial perpendicular anisotropy (iPMA) which was discovered to exist at the interface between magnetic transition metals and oxides (Monso et al., 2002). iPMA was subsequently observed with a large variety of amorphous and crystalline oxides as well as with various transition metals such as Co, $\mathrm{Fe}$, and $\mathrm{CoFe}$ or $\mathrm{CoFeB}$ alloys (Rodmacq et al., 2003, 2009; Manchon et al., 2008; Nistor et al., 2009). This is a remarkable phenomenon considering that the amplitude of the anisotropy measured is on the scale of that found at $\mathrm{Co} / \mathrm{Pt}$ interfaces $\left(\sim 1.4 \mathrm{erg} / \mathrm{cm}^{2}=1.4 \mathrm{~mJ} / \mathrm{m}^{2}\right)$ even though all the elements involved ( $\mathrm{Co}, \mathrm{Fe}, \mathrm{Al}, \mathrm{Mg}, \mathrm{O}$, etc.) are rather light and therefore have weak spin-orbit interactions. Detailed physicochemical characterizations [x-ray absorption (XAS) and x-ray photoemission (XPS)] revealed that this interfacial anisotropy was produced by the formation of chemical bonds between the oxygen ions of the oxide and the ions of the adjacent transition metal (Manchon et al., 2008; Rodmacq et al., 2009). Ab initio calculations later confirmed that hybridization between the $s p$-oxygen orbitals and the $d z^{2}$ orbitals in the transition metal ( $\mathrm{Co}$ or $\mathrm{Fe}$ ) and the ensuing decrease in degeneracy in the transition metal $d$ orbitals cause this anisotropy (Yang et al., 2011). Ikeda et al. (2010) reported that this anisotropy could be used to prepare MgO-based out-of-plane magnetized tunnel junctions for spin-transfer-torque magnetic random access memories (STT-MRAM) exhibiting large TMR thanks to good crystallinity combined with low critical current for switching by STT thanks to a low Gilbert damping. These observations triggered considerable interest. Indeed, in the context of STT-MRAM development, out-of-plane magnetized MTJs have been shown to offer much better scale-down capacity than their in-plane counterparts. Indeed, the PMA can be much larger than in-plane anisotropy (IMA) in sputtered films. In-plane anisotropy in sputtered films of practical interest for MRAM is due to shape anisotropy provided by giving the MRAM cell an elongated shape but this yields a typical anisotropy field in the 100-200Oe. In contrast, PMA can yield an effective anisotropy field of several kOe. This capacity explains why today most R\&D efforts devoted to MRAM focus on these out-of-plane magnetized MTJs combining high anisotropy and low damping. Major microelectronics companies are now actively working on the development of STT-MRAM which is expected to be scalable to, and beyond, the 16-nm node.

This phenomenon of iPMA at magnetic transition metal/ oxide interfaces is therefore of great interest not only from a basic research point of view, but also for applications, in particular, magnetic memories.

Several other research areas have recently emerged that take advantage of this iPMA, and they have become active research topics. One example is voltage control of magnetism in metallic systems (Weisheit et al., 2007; Shiota et al., 2012), which could pave the way toward very low-power nonvolatile memories and logic devices. Along these lines, the iPMA at magnetic metal/ oxide interfaces was found to be tunable by applying a voltage across the oxide layer (Schellekens et al., 2012; Shiota et al., 2009). Even though the magnetic layer is metallic, the electrical field can penetrate the magnetic layer to a depth corresponding to the Thomas-Fermi screening length, to induce effects on the electronic density of states (DOS) around the interface. Since iPMA is interfacial in origin, this effect is large enough to yield a significant variation in iPMA as a function of the bias voltage. This creates a unique way to manipulate the magnetic properties in thin films by applying voltage rather than current, and, in particular, a new means to switch magnetization through the use of voltage pulses in memory devices (Shiota et al., 2012). 
In contrast to out-of-plane magnetized $\mathrm{Co} / \mathrm{Pt}$-based multilayers, films based on $\mathrm{Co}(\mathrm{Fe})$ /oxide interfaces were observed to exhibit much lower magnetization pinning. Thus, despite their very large out-of-plane anisotropy, their coercivity, a few $\mathrm{Oe}$, is much weaker than that of $(\mathrm{Co} / \mathrm{Pt})$ multilayers of several $\mathrm{kOe}$. This low coercivity makes them interesting for studies of domain wall (DW) propagation in out-of-plane magnetized systems. Indeed, record domain wall speeds were obtained in this type of system (Miron et al., 2011; Schellekens et al., 2012), and they could therefore be good candidates for racetrack memories (Parkin, Hayashi, and Thomas, 2008).

In addition, the intrinsic electrical field which exists at the magnetic metal/oxide interface (Ibrahim et al., 2016b) due to charge transfer between the metal and the oxygen atoms in the oxide produces an interfacial Rashba effect. This effect can be harnessed to switch the magnetization of the magnetic metallic layer or to generate steady state magnetic excitations with an in-plane current in three-terminal magnetic tunnel junctions (Miron, Garello et al., 2011). Both Rashba and SHE (Liu, Pai et al., 2012) yield SOT which can be used to design three-terminal devices with the potential to become ultrafast and low-power memories and logic devices with infinite endurance.

The remainder of this review is organized as follows: Sec. II deals with the discovery of iPMA at magnetic metal/oxide interfaces and early studies of the phenomenon. Ab initio theoretical studies of the origin of this iPMA are covered in Sec. III. Section IV discusses the use of this iPMA in perpendicular STT-MRAM and the resulting scalability of these memories. Emerging research directions that take advantage of this phenomenon are dealt with in Sec. V, which covers the voltage control of the iPMA, and Sec. VI, where some other recent R\&D topics taking advantage of this phenomenon are considered.

\section{PMA AT MAGNETIC TRANSITION METAL/OXIDE INTERFACES: A HISTORICAL PERSPECTIVE}

\section{A. First observation with AlOx formed by rf oxidation and natural oxidation}

iPMA at magnetic transition metal/oxide interfaces was first observed in 2002 in a study of the oxidation of an $\mathrm{Al}$ layer to form an alumina-based magnetic tunnel junction (Monso et al., 2002). Natural oxidation and plasma oxidation were both used and results were very similar. The samples investigated consisted of a Pt underlayer surmounted with Co $0.6 \mathrm{~nm} / \mathrm{Al} t_{\mathrm{Al}}$. Following oxidation, the out-of-plane magnetic hysteresis loop was measured based on the extraordinary Hall effect (EHE) (also called the anomalous Hall effect) with magnetic field applied perpendicular to the plane of the sample. The EHE is sensitive to the out-of-plane component of magnetization. Figure 1 illustrates the effects observed for a 1.5-nm-thick Al layer exposed to an oxygen plasma $\left(5 \times 10^{-2}\right.$ mbar with a rf power of $0.4 \mathrm{~W} / \mathrm{cm}^{2}$ ). During oxidation, the oxygen penetrates inside the $\mathrm{Al}$ layer by diffusing along the grain boundaries, gradually gaining access to the inner part of the grains (Bae et al., 2002). Thus the $\mathrm{Al}$ is progressively transformed into amorphous alumina. After an adequate duration of exposure to the oxygen plasma, the Al layer becomes fully oxidized. If oxidation

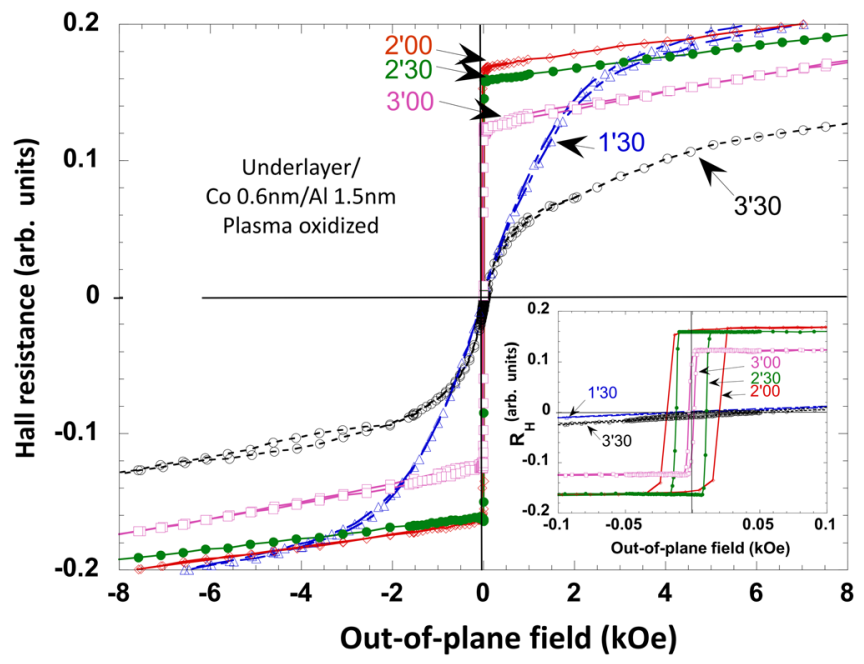

FIG. 1. Out-of-plane hysteresis loops measured for Pt $3 \mathrm{~nm} / \mathrm{Co}$ $0.6 \mathrm{~nm} / \mathrm{Al} 1.5 \mathrm{~nm}$ based on EHE after various durations of exposure to an oxygen plasma. From Rodmacq et al., 2003.

continues beyond this point, the oxygen starts to penetrate the underlying magnetic electrode where it diffuses inside the Co layer through the grain boundaries, leading to overoxidation of the barrier. Figure 1 shows the magnetic response of the underlying magnetic electrode with various durations of exposure to the oxygen plasma, as measured by EHE. For short durations (less than $2 \mathrm{~min}$, for example, $1 \mathrm{~min} 30 \mathrm{~s}$ ), a slanted hysteresis loop is observed with zero remanence and a relatively large saturation field of about $5 \mathrm{kOe}$. In these conditions, the Co anisotropy is in plane despite the use of a Pt underlayer, indicating that the interfacial perpendicular anisotropy provided by the Pt layer is too weak in these experiments to overcome the easy-plane demagnetizing energy of the Co layer. With oxygen exposure lasting $2 \mathrm{~min}$, a square hysteresis loop is observed, with $100 \%$ remanence and a low coercive field of about 20 Oe (see the inset in Fig. 1). These parameters indicate that the anisotropy of the Co layer has clearly changed and is now out of plane even though the amplitude of the EHE is the same as in the underoxidized sample, indicating that the Co layer is not yet oxidized. Since the oxygen penetrates from the upper surface, Monso et al. (2002) hypothesized that an interfacial anisotropy appeared at the $\mathrm{Co} /$ alumina interface as a result of the oxidation process. This hypothesis was later confirmed by XAS and XPS experiments. When the film is further oxidized, the square shape of the hysteresis loop, and thus the interfacial perpendicular anisotropy, is maintained up to $3 \mathrm{~min}$ of oxidation time, although a drop in the EHE amplitude is observed indicating that the Co starts to become oxidized, probably due to diffusion of the oxygen along the grain boundaries (Bae, Shin, and Lee, 2002). Finally, for oxidation durations exceeding $3 \mathrm{~min}$, the hysteresis loop becomes slanted with a high saturation field indicating that perpendicular anisotropy is lost. The loss of perpendicular anisotropy is therefore associated with the disappearance of the $\mathrm{Co} /$ alumina interface and formation of a $\mathrm{Co} / \mathrm{CoO} /$ alumina bottom electrode.

These effects were subsequently reproduced for various thicknesses of the Al capping layer (Rodmacq et al., 2003). The dramatic variation in out-of-plane susceptibility of the Co layer induced by the oxidation of $\mathrm{Al}$ into alumina and the 
B. Dieny and M. Chshiev: Perpendicular magnetic anisotropy at ...

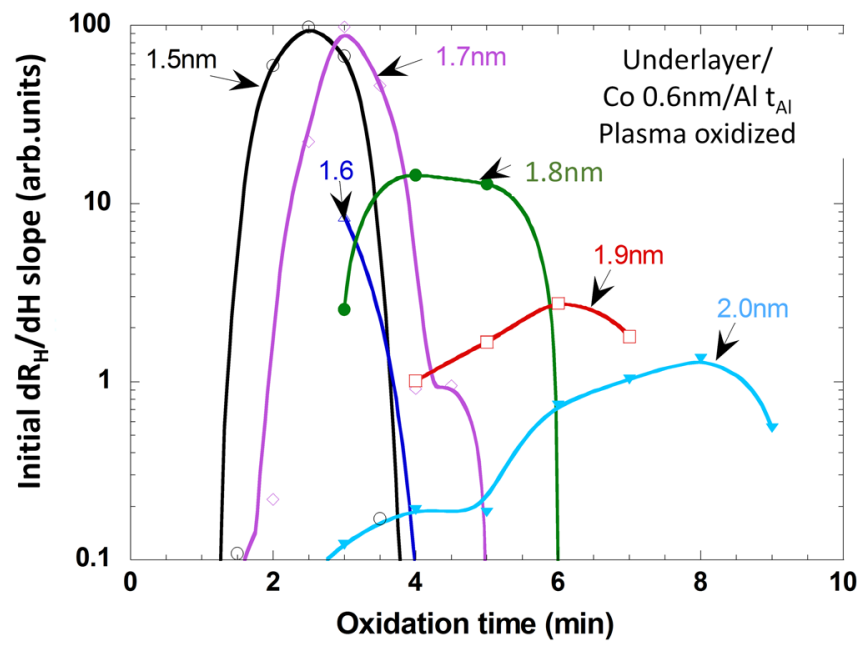

FIG. 2. Out-of-plane susceptibility of Pt $3 \mathrm{~nm} / \mathrm{Co} 0.6 \mathrm{~nm} / \mathrm{Al}$ $t_{\mathrm{Al}}$ measured based on EHE as a function of the duration of exposure to the oxygen plasma for various $\mathrm{Al}$ layer thicknesses. From Rodmacq et al., 2003.

associated increase in $\mathrm{Co} / \mathrm{AlOx}$ interfacial anisotropy can be clearly observed when the low-field slope of the EHE hysteresis loops is plotted against time (Fig. 2). This representation reveals changes to the out-of-plane susceptibility of 3 orders of magnitude. The maximum out-of-plane susceptibility is associated with the optimal barrier oxidation conditions, corresponding to the situation where the oxygen atoms reach the $\mathrm{Co} / \mathrm{Al}$ interface transforming it into a Co/alumina interface. Taking advantage of this correlation, it was proposed that the measurement of the out-of-plane susceptibility could be used to control and optimize the oxidation of tunnel barriers in MTJs (Monso et al., 2002), a technique which could be implemented in situ during growth using the magneto-optic Kerr effect (MOKE). If the thickness of the Al capping layer is varied, the optimum plasma oxidation time shifts toward longer times (Fig. 2). This shift could be readily explained by considering that the thicker the barrier, the longer it will take for the oxygen to reach the underlying Co interface. In parallel to this increased penetration time, the maximum in EHE slope becomes broader indicating that the oxidation process is less homogeneous as the $\mathrm{Al}$ thickness increases.

Similar trends were observed using natural oxidation of the $\mathrm{Al}$ layer and a CoFe bottom electrode instead of a Co one (Monso et al., 2002).

\section{B. A general phenomenon at magnetic metal/oxide interface:}

Soon after, SPINTEC demonstrated that this phenomenon is quite common and can be observed with a large variety of amorphous or crystalline oxides which may be formed from the metallic element by natural oxidation or by plasma oxidation, or even when the oxide layer is directly rf sputtered from an oxide target. The phenomenon is therefore relatively robust. This iPMA is already present in as-deposited samples, but can be maximized by annealing the structure (see Sec. II.E). As an example, Fig. 3 shows a similar crossover of anisotropy to that illustrated in Fig. 1, but for a $\mathrm{CrOx}$

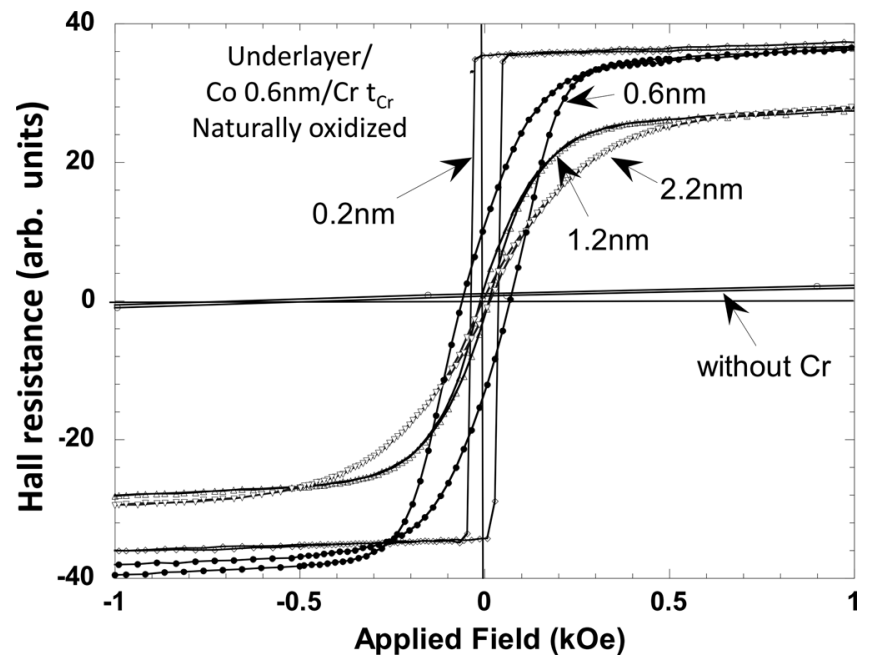

FIG. 3. EHE hysteresis loops for a series of Cr-capped samples naturally oxidized by exposure to air, measured with a field applied out of plane. Sample composition: $\mathrm{Pt} 3 \mathrm{~nm} / \mathrm{Co}$ $0.6 \mathrm{~nm} / \mathrm{Cr} t_{\mathrm{Cr}}$.

barrier. For this figure, the samples consisted of a Pt underlayer surmounted by $\mathrm{Co} 0.6 \mathrm{~nm} / \mathrm{Cr} t_{\mathrm{Cr}}$, and the EHE loops were measured $12 \mathrm{~h}$ after deposition and exposure to air. For very thin $\mathrm{Cr}$ layers, full oxidization is achieved naturally by exposure to air, and the anisotropy is perpendicular. However, for thicker $\mathrm{Cr}$ layers, natural oxidation cannot fully oxidize the $\mathrm{Cr}$ down to the Co interface and therefore the perpendicular anisotropy disappears.

Figure 4 shows observations of the same maximum of out-of-plane susceptibility as illustrated in Fig. 2 for four different oxide materials formed by natural oxidation of the

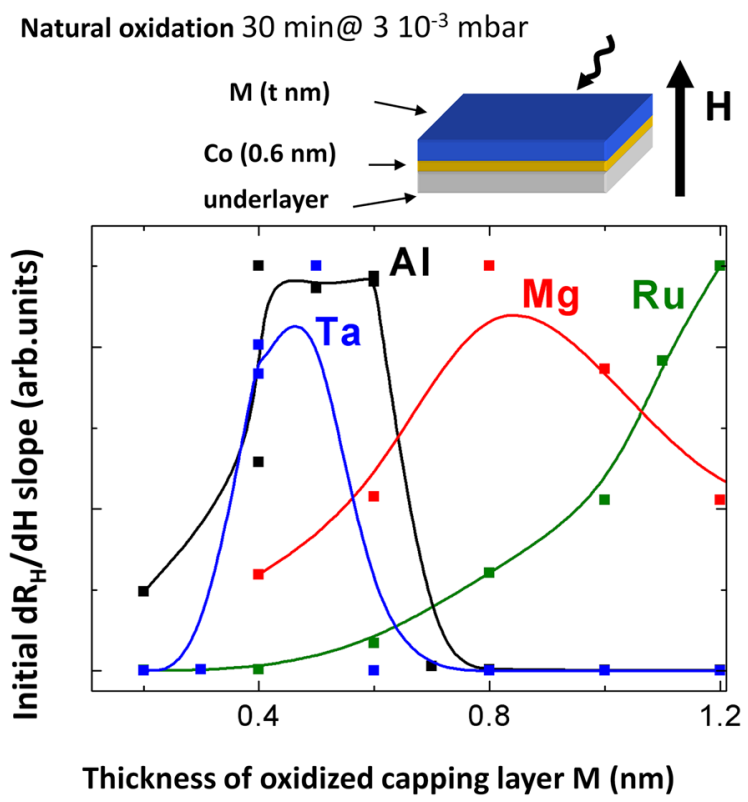

FIG. 4. Variation of the out-of-plane susceptibility for naturally oxidized samples composed of Pt $3 \mathrm{~nm} / \mathrm{Co} 0.6 \mathrm{~nm} / \mathrm{MOx} t_{M}$. The samples were naturally oxidized by exposure to oxygen at a partial pressure of 160 mbars for $30 \mathrm{~min}$. Samples were then capped by applying a protective Pt layer to avoid further oxidation due to air exposure. From Manchon et al., 2008. 
corresponding metallic element. Interestingly, some of these oxides are amorphous ( $\mathrm{AlOx}, \mathrm{TaOx})$, whereas others are polycrystalline $(\mathrm{MgO}, \mathrm{RuO})$. The optimum thickness of the metallic layer, allowing the maximum out-of-plane susceptibility to be reached, depends on the nature of the element. This effect can be explained in terms of the different kinetics of oxidation for the different oxides and the self-limiting oxidation which occurs with some oxides such as $\mathrm{TaOx}$ and $\mathrm{AlOx}$.

\section{Correlation between PMA and electron-specular reflection in specular spin valves}

The key role played by the degree of oxidation along the magnetic metal/oxide interface was further demonstrated by experiments performed in 2003 which found a correlation between the amplitude of the iPMA at the Co/AlOx interface and the degree of specular reflection at the same interface when the trilayer was inserted into current-in-plane (CIP) specular spin valves (Rodmacq et al., 2003). This correlation was established by carrying out two experiments in parallel. The first experiment measured perpendicular anisotropy at the Co/AlOx interface in Pt $3 \mathrm{~nm} / \mathrm{Co} 0.6 \mathrm{~nm} / \mathrm{Al} 1.5 \mathrm{~nm}$ sandwiches as a function of the duration of exposure to an oxygen plasma, as described before. The second experiment consisted of growing spin valves composed of Pt $3 \mathrm{~nm} / \mathrm{Co} 3 \mathrm{~nm} / \mathrm{Ru}$ $0.5 \mathrm{~nm} / \mathrm{Co} 2 \mathrm{~nm} / \mathrm{Cu} 3 \mathrm{~nm} / \mathrm{Co} 1.5 \mathrm{~nm} / \mathrm{Al} 1.5 \mathrm{~nm}$ (Dieny et al., 1991) and similarly measuring their CIP properties (resistance and GMR) as a function of the duration of exposure of the capping layer to the oxygen plasma. The same partial pressure of oxygen and rf power were used in the two experiments so that the two $\mathrm{Al}$ capping layers could be considered to be oxidized in identical conditions. In the second series of experiments, the gradual oxidation of $\mathrm{Al}$ to form $\mathrm{AlOx}$ changed the electron scattering pattern at the $\mathrm{Co} / \mathrm{AlOx}$ interface from diffuse scattering when the interface is poorly or not oxidized to specular reflection when the metal/ oxide interface forms a perfect wall of energy for electron conduction. Oxygen vacancies present at the interface can be qualitatively viewed as holes in this wall, and they will generate electron diffusion. When no more vacancies remain, electrons will be perfectly reflected by this interface, allowing them to conserve their momentum parallel to the interface. In these conditions, a minimum in CIP resistance and a corresponding maximum in GMR amplitude are measured due to the decreased scattering at the Co/AlOx interface (Egelhoff et al., 1997; Veloso et al., 2000; Da Costa et al., 2002). If the Al layer is oxidized beyond the optimal conditions, CIP resistance increases due to the additional resistance introduced by the oxidation along the Co grain boundaries. This increased resistance is associated with a decrease in GMR amplitude due to additional scattering and the reduced specular reflection caused by the inhomogeneous oxygen penetration into the Co layer (Rodmacq et al., 2003).

The data obtained for these two sets of experiments are reported in Fig. 5 as a function of the duration of exposure to the oxygen plasma. Clearly, the maximum perpendicular anisotropy is measured in the same oxidation conditions where the maximum specular reflection is obtained at the $\mathrm{Co} / \mathrm{AlOx}$ interface in specular spin valves. This maximum
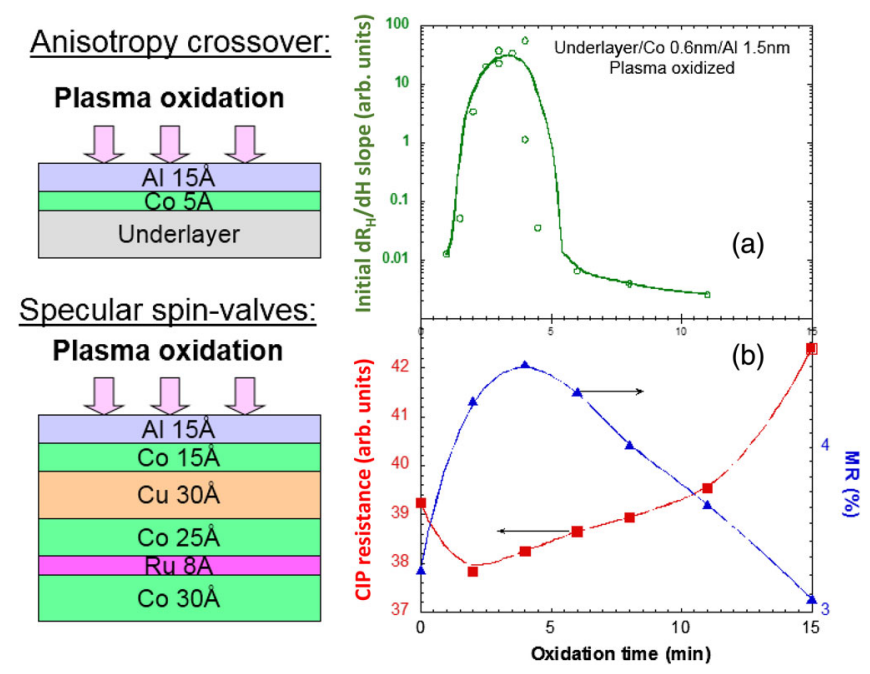

FIG. 5. Experimental observation of the correlation between the maximum iPMA at the $\mathrm{Co} / \mathrm{AlOx}$ interface in $\mathrm{Pt} / \mathrm{Co} / \mathrm{AlOx}$ and the maximum specular reflection in specular spin valves with a free $\mathrm{Co} / \mathrm{AlOx}$ layer, as a function of the degree of oxidation of the capping layer $(\mathrm{AlOx})$. These data confirm that the iPMA is maximized when the oxygen has reached the $\mathrm{Co} / \mathrm{AlOx}$ interface producing a very sharp energy wall for the conduction electrons at the metal/oxide interface which is then characterized by a very small number of oxygen vacancies. From Rodmacq et al., 2003.

specular reflection corresponds to the minimum CIP resistance and correlatively to the maximum CIP GMR. These correlations confirmed that the maximum iPMA is obtained when the oxygen ions have reached the $\mathrm{Co} / \mathrm{AlOx}$ interface, when no more metallic Al remains along the interface. This corresponds to the point when the density of oxygen vacancies along the interface has been drastically reduced.

\section{Physicochemical characterization (XAS, XPS) of the metal/ oxide interface and correlation with anisotropy}

To get a deeper insight into the physicochemical properties of the magnetic metal/oxide interface, XAS and photoemission experiments were performed on a series of $\mathrm{Pt} / \mathrm{Co} / M \mathrm{Ox}$ samples with $M=\mathrm{Al}, \mathrm{Mg}$, Ta, and $\mathrm{Ru}$. The results of these experiments are described in Figs. 6-8 for a Co/AlOx interface (Manchon et al., 2008). The samples studied were composed of an underlayer (Pt $3 \mathrm{~nm}$ ) surmounted by Co $0.6 \mathrm{~nm} / \mathrm{Al} 1.6 \mathrm{~nm}$. Samples were not annealed, they were oxidized using an oxygen rf plasma at a partial pressure of $3 \times 10^{-3} \mathrm{mbar}$ and an rf power of $10 \mathrm{~W}$. Figure 6 shows the EHE response of these samples when an out-of-plane field was applied after various durations of exposure to the oxygen plasma. The observations are consistent with those shown in Fig. 1.

Thus, for samples oxidized for durations shorter than $25 \mathrm{~s}$, the magnetization loops show no hysteresis and nearly zero remanence, indicating that the magnetic anisotropy is in plane [Fig. 6(b)]. With intermediate oxidation times, between 30 and $40 \mathrm{~s}$, square hysteresis loops were observed with sharp magnetization reversal and nonzero coercivity associated with out-of-plane anisotropy. When the duration of oxidation exceeded $45 \mathrm{~s}$, the hysteresis loops were once again slanted with zero remanence and large saturation fields. To quantify the 


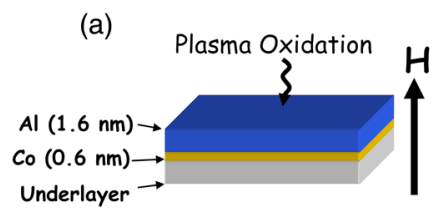

(c)

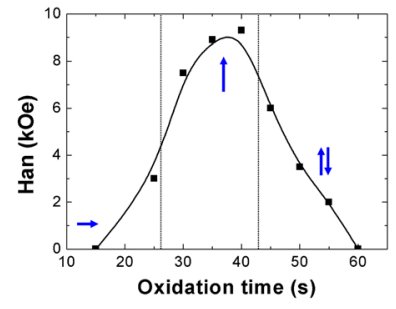

(b)

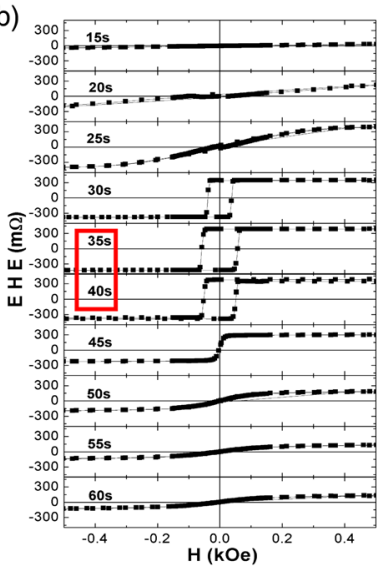

FIG. 6. (a) Sample geometry, (b) EHE vs out-of-plane field for various durations of exposure to the oxygen plasma, and (c) anisotropy field vs oxidation time determined from EHE measured while applying an in-plane field. From Manchon et al., 2008.

perpendicular anisotropy, the EHE was also measured with an in-plane field and the anisotropy field was derived from these measurements (Manchon et al., 2008); see Fig. 6(c). The maximum iPMA was observed with oxidation durations between 35 and $40 \mathrm{~s}$, when the hysteresis loops in Fig. 6(c) are the most square.

Interestingly, these measurements with in-plane field also revealed that the disappearance of the squareness of the

hysteresis loops after more than $45 \mathrm{~s}$ of oxidation is not immediately due to a loss of perpendicular anisotropy but to the fact that magnetization is split into up domains and down domains due to oxygen diffusing within the Co layer at the grain boundaries. This local Co oxidation disrupts the in-plane exchange stiffness and facilitates the formation of domain walls across the grain boundaries. In fact, the Co grains become almost uncoupled so that the magnetization can easily split into up domains and down domains to minimize the magnetostatic energy. This causes the hysteresis loop to become slanted.

Figures 7 and 8 show the XAS and XPS spectra obtained for the same series of samples. A direct correlation can therefore be established between the magnetic and physicochemical properties. XAS measurements were performed around the Co $L_{2,3}$ absorption edges (760-800 eV). Figure 7 shows the XAS spectra for $\mathrm{Pt} / \mathrm{Co} / \mathrm{AlOx}$ trilayers exposed to the oxygen plasma for different durations (hereafter called oxidation time for simplicity). XAS spectra for pure $\mathrm{Co}$ and $\mathrm{CoO}$ are shown as references in Figs. 7(a) and 7(c), respectively. For $t<25 \mathrm{~s}$ [Fig. 7(a)], the spectra for the trilayer resemble those for pure Co. With $30<t<40 \mathrm{~s}$ [Fig. 7(b)], shoulders appear in the absorption spectra indicating the presence of small contributions due to the formation of $\mathrm{CoO}$. At $45<t<60 \mathrm{~s}$ [Fig. 7(c)] these shoulders become very clear and the absorption spectra strongly resemble that for pure $\mathrm{CoO}$.

Figures 7(d)-7(f) show the derivatives of the XAS spectra. These derivatives more clearly reveal the presence of $\mathrm{CoO}$ in the Co layer, and the vertical lines indicate the energy of the

\section{XAS spectra at $C_{0} L_{2,3}$ edges}

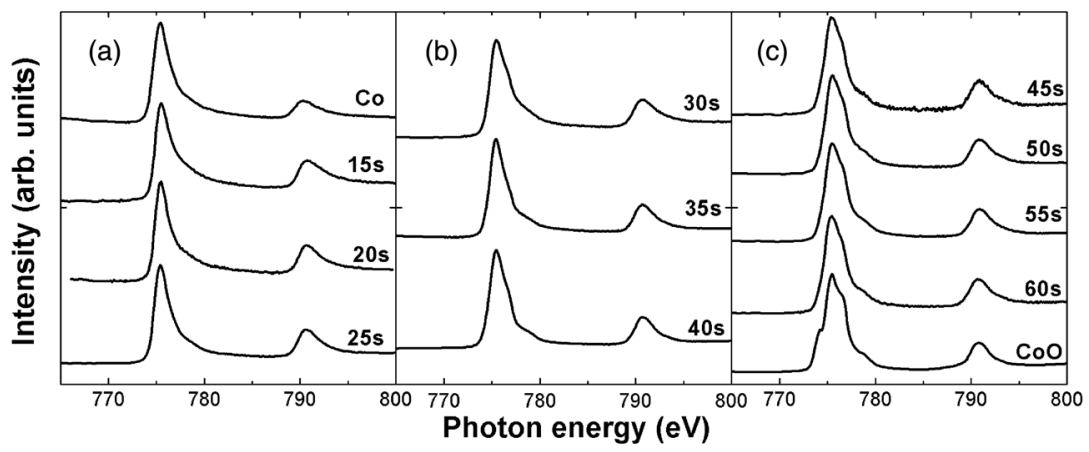

Derivatives of XAS spectra at Co L2,3 edges

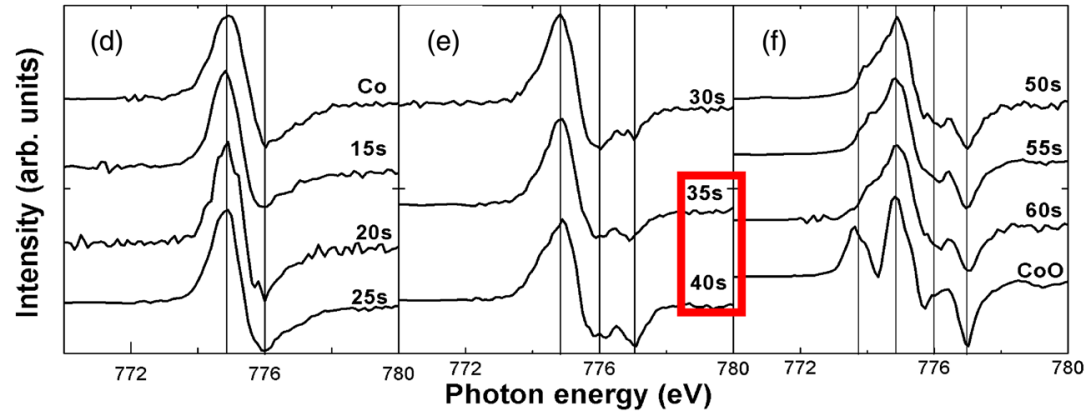

FIG. 7. XAS spectra measured for $\mathrm{Co} L_{2,3}$ within $\mathrm{Pt} / \mathrm{Co} / \mathrm{AlOx}$ trilayers for (a) underoxidized, (b) optimally oxidized, and (c) overoxidized samples, and their corresponding derivatives (d), (e), and (f) at the Co $L_{3}$ edge. Reference spectra for pure Co are shown in (a) and (d) and for $\mathrm{CoO}$ in (c) and (f). The solid and dotted vertical lines indicate the energy positions of the shoulders in the XAS spectra due to the presence of Co and CoO. From Manchon et al., 2008. 


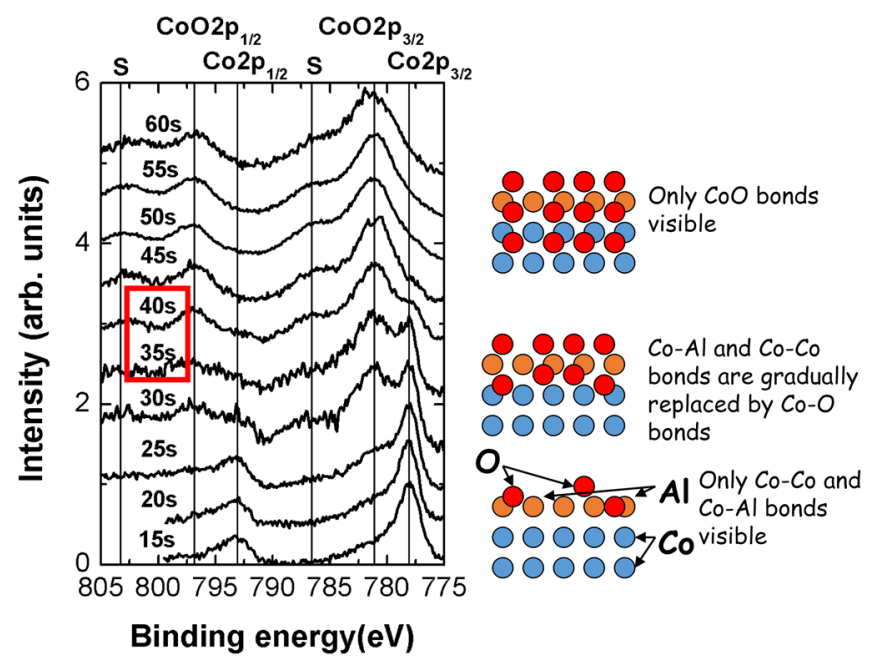

FIG. 8. Co $2 p$ XPS spectra for $\mathrm{Pt} 3 \mathrm{~nm} / \mathrm{Co}, 0.6 \mathrm{~nm} / \mathrm{Al}$, $1.6 \mathrm{~nm}+$ rf-plasma oxidation for various oxidation times. Right: illustration of how the oxygen atoms penetrate the structure as the oxidation time increases. From Manchon et al., 2008.

peaks on the derivative corresponding to Co (solid line) and $\mathrm{CoO}$ (dashed line). For short oxidation times [Fig. 7(d)] only peaks corresponding to pure $\mathrm{Co}$ are present. At intermediate oxidation times [Fig. 7(e)] a small peak appears at $777 \mathrm{eV}$, corresponding to a small proportion of $\mathrm{CoO}$. Finally, for long oxidation times [Fig. 7(f)], two $\mathrm{CoO}$ peaks are present, although they remain smaller than the peaks detected with pure Co. These observations clearly indicate that no oxygen atoms are present in the Co layer after short oxidation times. Some oxidation, probably near the interface, appears at intermediate oxidation times. And finally, after considerable exposure, oxygen penetrates deeply into the Co layer.

To obtain further information on the chemical composition of the Co layer, XPS measurements were performed setting the beam line energy to $h \nu=1130 \mathrm{eV}$ to probe Co $2 p$ levels (Manchon et al., 2008); see Fig. 8. It should be remembered that XPS is surface sensitive, so that the spectra provide information on the chemical composition of the $\mathrm{Co}$ monolayer (ML) which is directly in contact with the AlOx layer.

Figure 8 shows the Co $2 p$ spectra obtained for the same series of samples as in Figs. 6 and 7. The spectrum for the $t=60 \mathrm{~s}$ sample is very similar to that obtained for pure $\mathrm{CoO}$ (Grimbolt, Bonnelle, and Beaufils, 1976; Carson, Nassir, and Langell, 1996). The two main peaks (lying at 796.3 and $781.1 \mathrm{eV}$ ) correspond to $\mathrm{CoO} 2 p_{1 / 2}$ and $\mathrm{CoO} 2 p_{3 / 2}$ core levels, respectively, while the two satellite peaks (denoted $S$, with positions at 803.3 and $786.7 \mathrm{eV}$ ) are produced by the charge transfer between $\mathrm{O} 2 p$ and Co $3 d$, respectively (Grimbolt, Bonnelle, and Beaufils, 1976; Carson, Nassir, and Langell, 1996). The spectrum for the $t=15 \mathrm{~s}$ sample is similar to that of pure cobalt (Sicot et al., 2005) with Co $2 p_{1 / 2}$ and Co $2 p_{3 / 2}$ detected at binding energies of 792.5 and $778.1 \mathrm{eV}$, respectively. This spectrum clearly shows that Co is completely unoxidized at $t=15 \mathrm{~s}$. At $t=20$ and $25 \mathrm{~s}$, a small contribution of Co-O bonds appears. Between $t=30$ and $40 \mathrm{~s}$, both $\mathrm{Co}-\mathrm{O}$ and $\mathrm{Co}-\mathrm{Co}$ bonds coexist, while for oxidation times exceeding $45 \mathrm{~s}$, the Co contribution has completely disappeared.

These XPS spectra therefore clearly show that the composition at the $\mathrm{Co} / \mathrm{AlOx}$ interface changes from quasipure Co to $\mathrm{CoO}$ during oxidation and that the maximum iPMA is obtained in conditions where $\mathrm{Co}-\mathrm{O}$ bonds have formed across the $\mathrm{Co} / \mathrm{AlOx}$ interface, but where the topmost interfacial $\mathrm{Co}$ layer is not yet oxidized. In these conditions, in the $\mathrm{Co}$ monolayer in contact with the AlOx oxide layer, Co-Co bonds still prevail, whereas $\mathrm{Co}-\mathrm{O}$ bonds prevail across the interface.

These observations, together with the fact that the maximum iPMA correlates with the maximum specular reflection, indicate that IPMA arises from the hybridization effect that occurs across the Co-oxide interface between the Co orbitals (particularly out-of-plane orbitals $d_{x z}, d_{y z}$, and $d_{z^{2}}$ ) and the $s p$ $\mathrm{O}$ orbitals. This hybridization eliminates the degeneracy between the magnetic transition metal $3 d$ orbitals pointing out of plane and those with planar symmetry $\left(d_{x y}\right.$ and $\left.d_{x^{2}-y^{2}}\right)$ giving rise in a first approximation to a crystalline field

$$
\Delta_{c}=E\left(y z, z x, z^{2}\right)-E\left(x y, x^{2}-y^{2}\right) .
$$

This crystalline field can yield a relatively large surface or interfacial anisotropy even in materials where spin-orbit coupling is relatively weak (Bruno, 1989). The amplitude of the perpendicular anisotropy correlates with the anisotropy in the magnetic transition metal orbital momentum; the PMA energy can be expressed as

$$
\Delta E_{\mathrm{PMA}}=\frac{G}{H} \frac{\xi}{4 \mu_{B}}\left(m_{\mathrm{orb}}^{\perp}-m_{\mathrm{orb}}^{/ /}\right),
$$

where $m_{\text {orb }}^{\perp(/ /)}$ is the out-of-plane (respectively, in-plane) orbital moment, $\xi$ is the spin-orbit-coupling parameter, and $G / H$ are band-structure-dependent coefficients (Bruno, 1989; Manchon et al., 2008). Note that Bruno's model is approximate and valid only with large exchange splitting and when off-site and/or spin-mixing spin-orbit-coupling are negligible (van der Laan, 1998; Andersson et al., 2007; Li et al., 2013). However, it can still be used to qualitatively determine the magnetic anisotropy arising from the coupling between the anisotropy of the $3 d$ orbital moment $\vec{l}$ and the magnetic moment $\vec{s}$ of the magnetic atoms through the spin-orbit coupling $\xi \cdot \vec{l} \cdot \vec{s}$. This interpretation of the origin of the iPMA at interfaces between transition magnetic metals (Co, $\mathrm{Fe}, \mathrm{CoFeB}$ ) and oxides (AlOx, MgO, HfOx, CrOx, TaOx) was later confirmed by $a b$ initio calculations (Nakamura et al., 2010; Shimabukuro et al., 2010; Yang et al., 2011; Liang et al., 2014; see Sec. III).

X-ray magnetic circular dichroism (XMCD) experiments performed on $\mathrm{MgO} / \mathrm{CoFeB}$ interfaces (Tsai et al., 2012) and on $\mathrm{Fe} / \mathrm{MgO}$ interfaces (Okabayashi et al., 2014) also confirmed this interpretation. As with the $\mathrm{Co} / \mathrm{AlOx}$ samples described, Okabayashi et al. observed the existence of extra prepeaks in the XAS spectra for the $\mathrm{O} K$ edge. These supplementary peaks clearly indicate that $\mathrm{Fe}-\mathrm{O}$ bonding occurs at the $\mathrm{MgO} / \mathrm{CoFeB}(\mathrm{Fe} / \mathrm{MgO})$ interfaces, leading to the hybridization of $\mathrm{Fe}$ and $\mathrm{O}$ orbitals. Furthermore, the strength of the PMA in annealed samples correlated with 
the orbital hybridization of $\mathrm{Fe}-3 d$ and $\mathrm{O}-2 p$ orbitals, as assessed using the $\mathrm{Fe}$ orbital moment determined from XMCD measurements.

\section{E. Influence of annealing}

The influence of annealing on iPMA at magnetic transition metal/oxide interfaces was thoroughly studied for the first time in 2009 (Rodmacq et al., 2009). In their work, Rodmacq et al. studied a series of samples composed of Pt $3 \mathrm{~nm} / \mathrm{Co}$, $0.6 \mathrm{~nm} / \mathrm{Al} 1.6 \mathrm{~nm}$ after rf-plasma oxidization of the Al layer for various oxidation times covering the whole range from underoxidized to overoxidized. The samples were subsequently annealed at various temperatures up to $450{ }^{\circ} \mathrm{C}$. To conveniently represent the data obtained, the magnetization reversal nucleation field was determined from the Hall hysteresis loops. This nucleation field $\left(H_{\text {nuc }}\right)$ is the characteristic field at which the magnetization is no longer saturated and starts to switch directions. When a decrease from positive saturation occurs, the nucleation field will be positive for samples with in-plane magnetization ( $0 \%$ remanence) and negative for samples with out-of-plane magnetization $(\sim 100 \%$ remanent magnetization). To conveniently describe the variations of $H_{\text {nuc }}$ over several orders of magnitude, the following notation was introduced: $H^{*}=\log \left(\left|H_{\text {nuc }}\right|\right)$, where $H^{*}$ will have the same sign as $H_{\text {nuc }}$, i.e., negative for out-of-plane magnetized samples and positive for in-plane magnetized samples. Figure 9(a) shows a color-coded illustration of $H^{*}$ plotted as a function of annealing temperature $\left(100{ }^{\circ} \mathrm{C}\right.$ to $450{ }^{\circ} \mathrm{C}$ ) and oxidation time (oxidation by exposure of the 1.6-nm Al capping layer to an rf oxygen plasma with exposure times between 15 and $60 \mathrm{~s}$ ). At low annealing temperatures, similar results to those described for annealed samples are obtained. An optimum oxidation time of around 35 to $40 \mathrm{~s}$ is observed, where the nucleation field is maximized along with the iPMA, as previously explained. In as-deposited or weakly annealed samples, the range of oxidation times over which iPMA is observed is relatively narrow [dark (blue) zone in Fig. 9(a)]. However, it is interesting to note that upon annealing this region broadens, suggesting that the oxygen migrates to the $\mathrm{Co} / \mathrm{AlOx}$ interface to allow its perfect oxidization and the formation of $\mathrm{Co}-\mathrm{O}-\mathrm{Al}$ bonds all over the interface.

This hypothesis was upheld by three other observations: (i) Fig. 9(b) (Manchon et al., 2008) shows the ratio of $\mathrm{Al}$ ions bonded to oxygen normalized for the total number of $\mathrm{Al}$ ions in the AlOx layer, as measured by XAS. This ratio is plotted against the initial oxidation time. Since XAS is sensitive to the bulk of the layer, the data clearly indicate that some of the oxygen has disappeared from the bulk of the layer following annealing at $350{ }^{\circ} \mathrm{C}$. (ii) In parallel, XPS data (Manchon et al., 2008) indicate that annealing increases the number of interfacial $\mathrm{Co}$ atoms bound to $\mathrm{O}$. (iii) The Hall angle reaches a maximum value in the region corresponding to high annealing temperatures and low to moderate oxidation times [not shown, Fig. 4 in

(a)

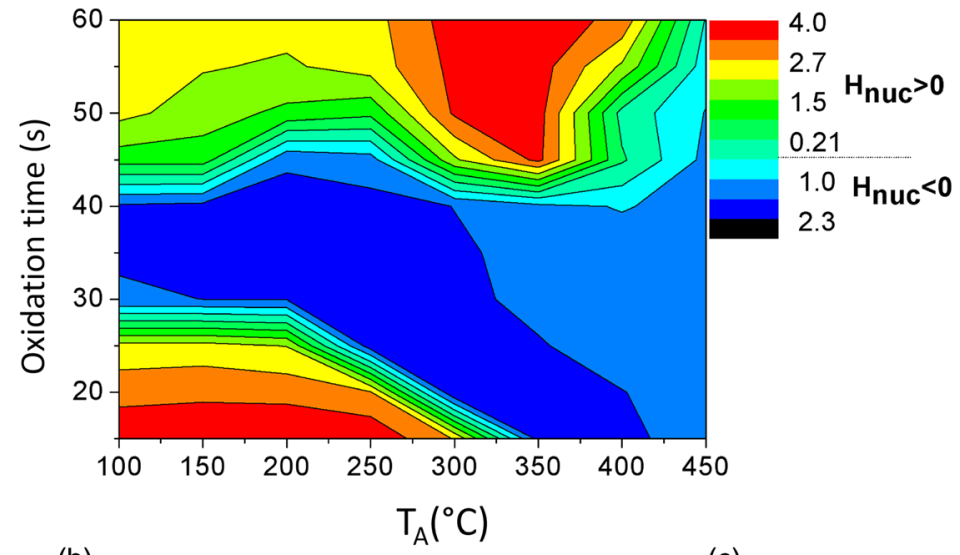

(b)

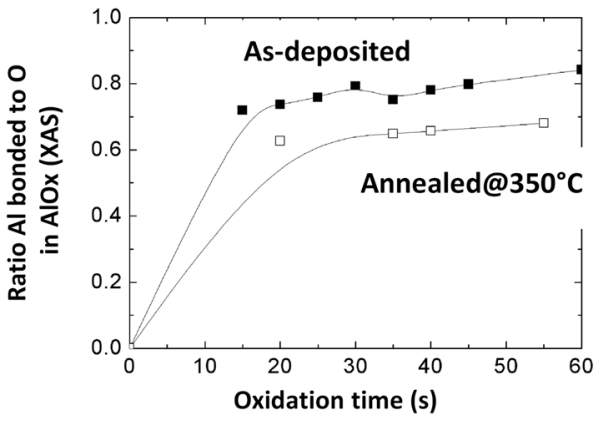

(c)

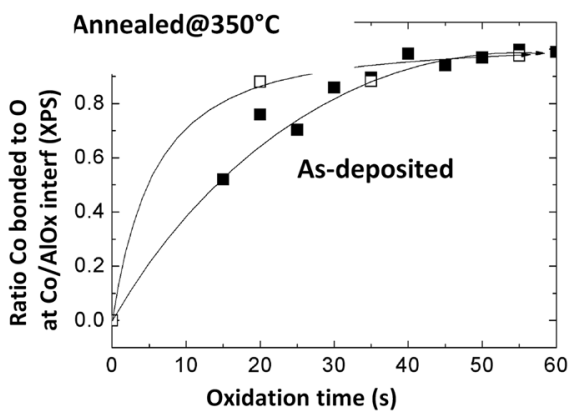

FIG. 9. (a) $H^{*}$ (see text) (color code) plotted as a function of annealing temperature $\left(T_{A}\right)$ and initial oxidation time for samples composed of Pt $3 \mathrm{~nm} / \mathrm{Co}, 0.6 \mathrm{~nm} / \mathrm{Al}, 1.6 \mathrm{~nm}$ oxidized by rf oxygen plasma. (b) Ratio of Al ions bonded to oxygen in the plasmaoxidized AlOx layer, as measured by XAS, vs oxidation time in as-deposited and annealed samples $\left(350{ }^{\circ} \mathrm{C}\right)$. From Manchon $e t$ al., 2008. (c) Ratio of Co ions bonded to oxygen at the Co/AlOx interface, as measured by XPS, vs initial oxidation time in as-deposited and annealed samples. From Manchon et al., 2008. 
Rodmacq et al. (2009)]. These observations can be explained if oxygen from the inner part of the AlOx layer or from the Co grain boundaries (for overoxidized samples) migrates toward the $\mathrm{Co} / \mathrm{AlOx}$ interface upon annealing, suggesting that this interface is the most stable location for oxygen ions. Note that similar results were obtained when $\mathrm{MgO}$ layers were substituted for AlOx layers (Manchon et al., 2008; Rodmacq et al., 2009).

The effective anisotropy field in samples with out-of-plane magnetization as well as the iPMA energy were extracted by measuring the EHE loops while applying an in-plane field to a series of plasma-oxidized samples composed of $\mathrm{Pt}$ $3 \mathrm{~nm} / \mathrm{Co}, t_{\mathrm{Co}} / \mathrm{Al} 1.6 \mathrm{~nm}$ after oxidation times ranging from 15 to $60 \mathrm{~s}$ and annealed at various temperatures between $100{ }^{\circ} \mathrm{C}$ and $450{ }^{\circ} \mathrm{C}$. The thickness of the Co layer varied between 0.5 and $3.8 \mathrm{~nm}$. Figure 10(a) shows the anisotropy field $\left(H_{\text {an }}\right)$ plotted as a function of annealing temperature and oxidation time for the series of samples with $t_{\mathrm{Co}}=0.6 \mathrm{~nm}$. The color scale is in kOe. Maximal PMA is clearly obtained at around $35 \mathrm{~s}$ oxidation time after $300^{\circ} \mathrm{C}$ annealing. In that region of the graph, superconducting quantum interference device (SQUID) measurements gave a value of $10^{3} \mathrm{emu} / \mathrm{cm}^{3}$ for the saturation magnetization of the Co layer. This value corresponds to an effective anisotropy constant
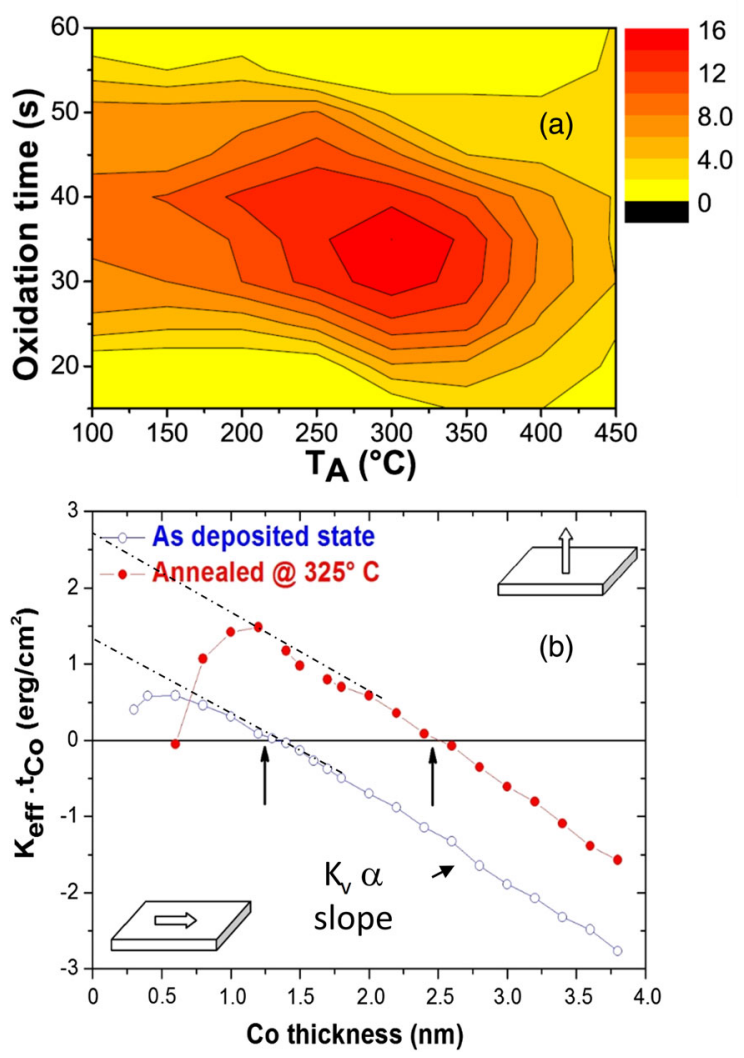

FIG. 10. (a) Anisotropy field map (in kOe) for Pt $3 \mathrm{~nm} / \mathrm{Co}$, $0.6 \mathrm{~nm} / \mathrm{Al} 1.6 \mathrm{~nm}$ samples, plasma oxidized for between 15 and $60 \mathrm{~s}$ and annealed at temperatures ranging from $100^{\circ} \mathrm{C}$ to $450{ }^{\circ} \mathrm{C}$. (b) Interfacial anisotropy in naturally oxidized samples composed of (thickness in $\mathrm{nm}$ ) $\mathrm{SiO}, 2 / \mathrm{Ta}, 3 / \mathrm{Pt}, 20 / \mathrm{Co}, t_{\mathrm{Co}} / \mathrm{Al}$, 0.5//Nat.Ox./Cu, 2/Pt 2, $\left(1 \mathrm{erg} / \mathrm{cm}^{2}=1 \mathrm{~mJ} / \mathrm{m}^{2}\right)$. From Rodmacq et al., 2009.
$K_{\text {eff }}=M_{s} H_{\text {an }} / 2$ of $0.8 \mathrm{MJ} / \mathrm{m}^{3}\left(8 \times 10^{6} \mathrm{erg} / \mathrm{cm}^{3}\right)$, which is larger than in most Pt- or Pd-based magnetic multilayers (Ikeda et al., 2005, 2008). In these oxidation and annealing conditions, the $\mathrm{Co} / \mathrm{AlOx}$ interface is optimally oxidized. As previously discussed, annealing promotes homogeneous oxidation along the interface, decreases roughness at the interface, modifies the $\mathrm{Pt} / \mathrm{Co}$ interface, and thus allows the sum of the two interfacial anisotropies at $\mathrm{Pt} / \mathrm{Co}$ and $\mathrm{Co} / \mathrm{AlOx}$ to largely overcome the demagnetizing energy. The underoxidation of the samples treated for oxidation times shorter than $25 \mathrm{~s}$ does not allow maximal PMA to be attained as the number of oxygen atoms at the $\mathrm{Co} / \mathrm{AlOx}$ interface is insufficient.

To extract the sum of the interfacial anisotropies at the $\mathrm{Pt} / \mathrm{Co}$ and $\mathrm{Co} / \mathrm{AlOx}$ interfaces, $K_{\text {eff }} t_{\mathrm{Co}}$ was plotted against $t_{\mathrm{Co}}$ in Fig. 10(b) for as-deposited and annealed samples. The slope of the linear part of these curves gives the effective bulk anisotropy which, here, is essentially the demagnetizing energy of the Co layer $\left(-\mu_{0} M_{s}{ }^{2} / 2\right)$. The intercept with the $y$ axis gives the sum of the interfacial anisotropy at the $\mathrm{Pt} / \mathrm{Co}$ and $\mathrm{Co} / \mathrm{AlOx}$ interfaces. Thus, after annealing at $325^{\circ} \mathrm{C}$ for $30 \mathrm{~min}$, an interfacial anisotropy of $K_{s}^{\mathrm{Pt} / \mathrm{Co}}+K_{s}^{\mathrm{Co} / \mathrm{AlOx}}=$ $2.8 \mathrm{~mJ} / \mathrm{m}^{2}\left(1 \mathrm{~mJ} / \mathrm{m}^{2}=1 \mathrm{erg} / \mathrm{cm}^{2}\right)$ is measured. Since the interfacial anisotropy at the $\mathrm{Pt} / \mathrm{Co}$ interface was previously reported to be in the range $0.6-1.4 \mathrm{~mJ} / \mathrm{m}^{2}$ (Guoa et al., 2006; Yakushiji et al., 2010), this result implies that the iPMA at the $\mathrm{Co} / \mathrm{AlOx}$ interface is in the range of $1.4-2.2 \mathrm{~mJ} / \mathrm{m}^{2}$, i.e., it is as large or even larger than that at the $\mathrm{Pt} / \mathrm{Co}$ interface.

A similar order of magnitude was obtained at $\mathrm{CoFe} / \mathrm{MgO}$ interfaces (Nistor et al., 2010). This order of magnitude was subsequently confirmed by various groups (Ma et al., 2012; Koo et al., 2013; Lambert et al., 2013) and by ab initio calculations (Niranjan et al., 2010; Yang et al., 2011). This result is remarkable considering how weak the spin orbit is in $\mathrm{Co}$ and AlOx. Indeed, a $\mathrm{Pt} / \mathrm{Co}$ interface is commonly considered to be the archetype of high interfacial anisotropy, and this property is assumed to rely on large spin-orbit coupling. Other interfaces such as $\mathrm{Co} / \mathrm{Ni}$ (Johnson et al., 1996; Gottwald et al., 2012) or Cu/Ni (Johnson et al., 1996) do exhibit perpendicular interfacial anisotropy with weak spin orbits, but their interfacial anisotropy is twofold to threefold weaker than that measured at $\mathrm{CoFe} /$ oxide or $(\mathrm{Pt} / \mathrm{Co})$ interfaces.

\section{F. Correlation between PMA and tunnel magnetoresistance in MgO-based MTJ}

A study carried out early in 2010 established a correlation between the amplitude of the interfacial PMA at $\mathrm{CoFe} / \mathrm{MgO}$ interfaces and the corresponding TMR in MTJ, where the tunnel barrier was prepared by applying natural oxidation conditions (Nistor et al., 2010). The structures used to investigate how anisotropy varied with $\mathrm{Mg}$ thickness were $\mathrm{SiO}_{2} / \mathrm{Ta} 3 / \mathrm{Pt} 30 / \mathrm{Co}_{70} \mathrm{Fe}_{30} 1 / \mathrm{Mg} t_{\mathrm{Mg}} / \mathrm{Ru} 5 \mathrm{~nm}$. The $\mathrm{Mg}$ layer was naturally oxidized to form $\mathrm{MgO}$ by exposing the metallic $\mathrm{Mg}$ layer to oxygen at a pressure of 1 mbar for $450 \mathrm{~s}$. The structures used to investigate the TMR amplitude were inplane magnetized MTJ stacks of composition: Ta 3/CuN 60/Ta 5/PtMn 20/ $\mathrm{Co}_{70} \mathrm{Fe}_{30} \quad 2 / \mathrm{Ru} \quad 0.74 / \mathrm{CoFeB} \quad 2 / \mathrm{Mg}$ 
$t_{\mathrm{Mg}} / \mathrm{CoFeB} 3 / \mathrm{Ta} 10$. The $\mathrm{Mg}$ layer was oxidized under the same conditions as those used for the first series of samples. Both series of samples were annealed at $\sim 330^{\circ} \mathrm{C}$ in $10^{-6}$ mbar vacuum for $1 \mathrm{~h}$ prior to characterization. Figure 11(a) shows the anisotropy fields for the out-of-plane magnetized first series of samples $(\mathrm{Pt} / \mathrm{CoFe} / / \mathrm{Mg} /$ natural oxidation based) alongside the TMR for the second series of samples, as measured by the CIP-TMR technique (Worledge and Trouilloud, 2003). The results of the two measurements clearly correlate, and the same $\mathrm{Mg}$ thickness produces maximal perpendicular anisotropy for the first series of samples and the maximum TMR in the second series. Since the samples were naturally oxidized, this means that these two maxima occur for the same degree of oxidation of the $\mathrm{CoFe} / \mathrm{MgO}$ interface. This observation implies that the same mechanism, i.e., the formation of $\mathrm{CoFe}-\mathrm{O}$ bonds at the
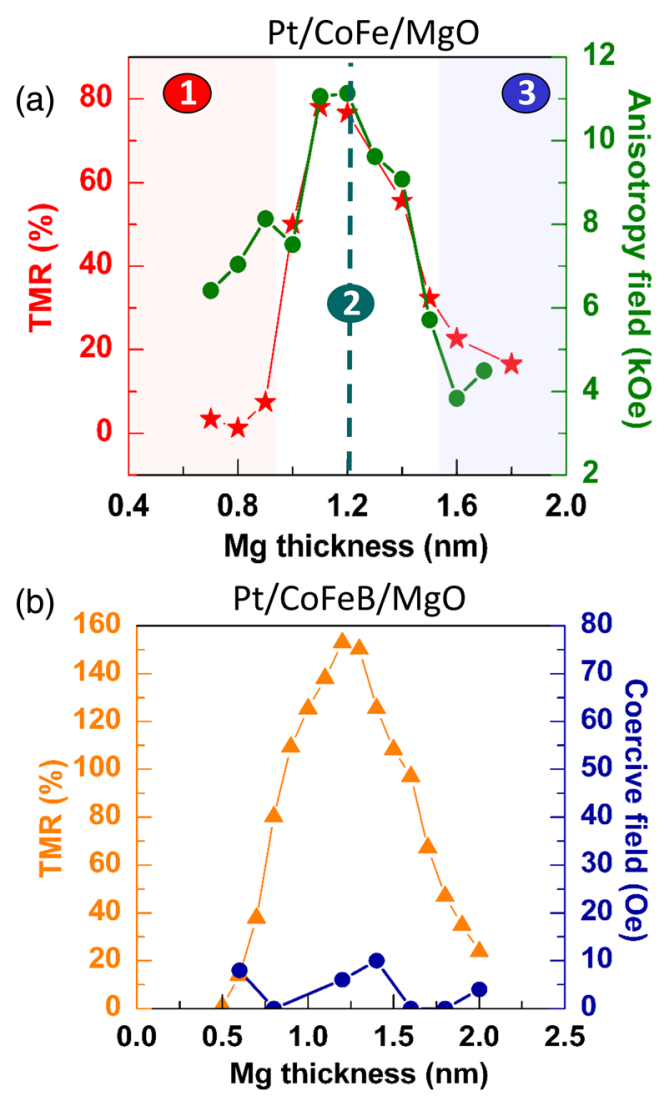

FIG. 11. (a) Variation in the TMR ratio in planar CoFeB-based MTJ and in the perpendicular anisotropy of a $\mathrm{Pt} / \mathrm{CoFe} / \mathrm{MgO}$ bottom electrode as a function of the thickness of the naturally oxidized $\mathrm{Mg}$ barrier. The MgO layers were prepared by natural oxidation in the same conditions for the two sample series. (b) Comparison of TMR in in-plane-magnetized CoFeB-based MTJ and of the anisotropy in $\mathrm{Pt} / \mathrm{CoFeB} 1.1 \mathrm{~nm} / \mathrm{MgO}$ bottom electrode for various $\mathrm{Mg}$ thicknesses. The bottom electrode contains B which the Pt buffer layer prevents from moving away from the $\mathrm{MgO}$ interface upon annealing. The presence of $\mathrm{B}$ along the $\mathrm{CoFeB} / \mathrm{MgO}$ interface inhibits the interfacial perpendicular anisotropy at $\mathrm{CoFeB} / \mathrm{MgO}$ so that the magnetization of the $\mathrm{CoFeB}$ layer remains in plane. The coercive field is plotted alongside the TMR amplitude; no correlation exists in this case. From Nistor et al., 2010. interface during oxidation, influences both the TMR amplitude and the interfacial anisotropy. This is consistent with the origin of the iPMA at magnetic transition metal/oxide interfaces in terms of hybridization between $\mathrm{O} p$ and $\mathrm{Co}(\mathrm{Fe}) d_{z^{2}}$ orbitals, as discussed, and also with the easier penetration of $\Delta_{1}$ electrons through the $\mathrm{MgO}$ barrier when it is $\mathrm{O}$ terminated (Butler et al., 2001)

A similar comparison was performed using $\mathrm{CoFeB}$ in place of CoFe [Fig. 11(b)] (Nistor et al., 2010). The main result of this comparison is that in $\mathrm{Pt} / \mathrm{CoFeB} 1.1 \mathrm{~nm} / \mathrm{MgO}$ magnetization lies in the plane of the film, whereas in $\mathrm{Pt} / \mathrm{CoFe}$ $1 \mathrm{~nm} / \mathrm{MgO}$ a strong out-of-plane anisotropy is observed. These experiments therefore demonstrated that the presence of $\mathrm{B}$ at the $\mathrm{CoFe}(\mathrm{B}) / \mathrm{MgO}$ interface considerably reduces the iPMA. The presence of boron prevents hybridization between the $\mathrm{Co}(\mathrm{Fe})$ orbitals and the oxygen $s p$ orbitals. The B is not absorbed away from the $\mathrm{MgO}$ interface upon annealing because in this experiment, the buffer layer $(\mathrm{Pt})$ is a noble metal (You et al., 2008; Mukherjee et al., 2009). Therefore the $\mathrm{B}$ remains in the $\mathrm{CoFeB}$ layer right next to the $\mathrm{CoFeB} / \mathrm{MgO}$ interface where it hampers PMA. Comparing the results presented in Figs. 11(a) and 11(b) shows how important it is to attract the $\mathrm{B}$ away from the $\mathrm{MgO}$ interface in $\mathrm{CoFeB} / \mathrm{MgO}$-based MTJ upon annealing so as to benefit from the interfacial PMA at the $\mathrm{CoFe} / \mathrm{MgO}$ interface while also maximizing the TMR amplitude (Nistor et al., 2010). Oh et al. (2014) subsequently confirmed this conclusion by comparing levels of iPMA in $\mathrm{CoFeB} / \mathrm{MgO}$ for structures associated with various underlayers including $\mathrm{Ta}$ and $\mathrm{Hf}$ (which attract $\mathrm{B}$ and therefore produce large PMA) and $\mathrm{Pt}$ and Pd (which are not good B getters and therefore do not promote interfacial PMA).

\section{G. First observations of PMA at Ta/CoFeB/MgO interfaces}

The next important step toward the use of this interfacial PMA in out-of-plane MTJ was achieved by Ikeda et al. (2010) at Tohoku University when they investigated out-of-plane magnetized $\mathrm{Ta} / \mathrm{CoFeB} / \mathrm{MgO}$-based MTJs. From a basic research point of view, this is a specific case of the various systems in which PMA was observed at transition metal/oxide interfaces since 2002. However, from a technical point of view, the results obtained by Ikeda et al. are quite important since they demonstrated that a large TMR and a large PMA with a relatively low STT switching current could be obtained in the same MTJ stacks. The idea for this study was as follows. The experiments by Nistor et al. (2010) pointed out the importance of attracting the $\mathrm{B}$ away from the $\mathrm{CoFeB} / \mathrm{MgO}$ interface to induce a strong PMA at this interface. Ikeda et al. did this by substituting a Ta underlayer for the Pt underlayer used by Nistor et al. Ta is known to be a good B getter, i.e., it is capable of drawing the B out from the initially amorphous $\mathrm{CoFeB}$ layer during postdeposition annealing of the MTJ (Yuasa and Djayaprawira, 2007; Kozina et al., 2010). Figure 12 shows the stacks used as well as some of the main results obtained. The MTJs investigated were composed of underlayer/Ta $5 / \mathrm{Co}_{20} \mathrm{Fe}_{60} \mathrm{~B}_{20} \quad\left(t_{\mathrm{CoFeB}}\right.$ from 1.0 to $1.3 \mathrm{~nm}) / \mathrm{MgO} \quad\left(t_{\mathrm{MgO}} 0.85\right.$ or $\left.0.9 \mathrm{~nm}\right) / \mathrm{Co}_{20} \mathrm{Fe}_{60} \mathrm{~B}_{20}$ (from 1.0 to $1.7 \mathrm{~nm}$ ) $/ \mathrm{Ta} 5 / \mathrm{Ru} 5$ (all thicknesses in nanometers) [Fig. 12(a)]. Electron-beam lithography and Ar-ion milling were used to produce circular sample devices 

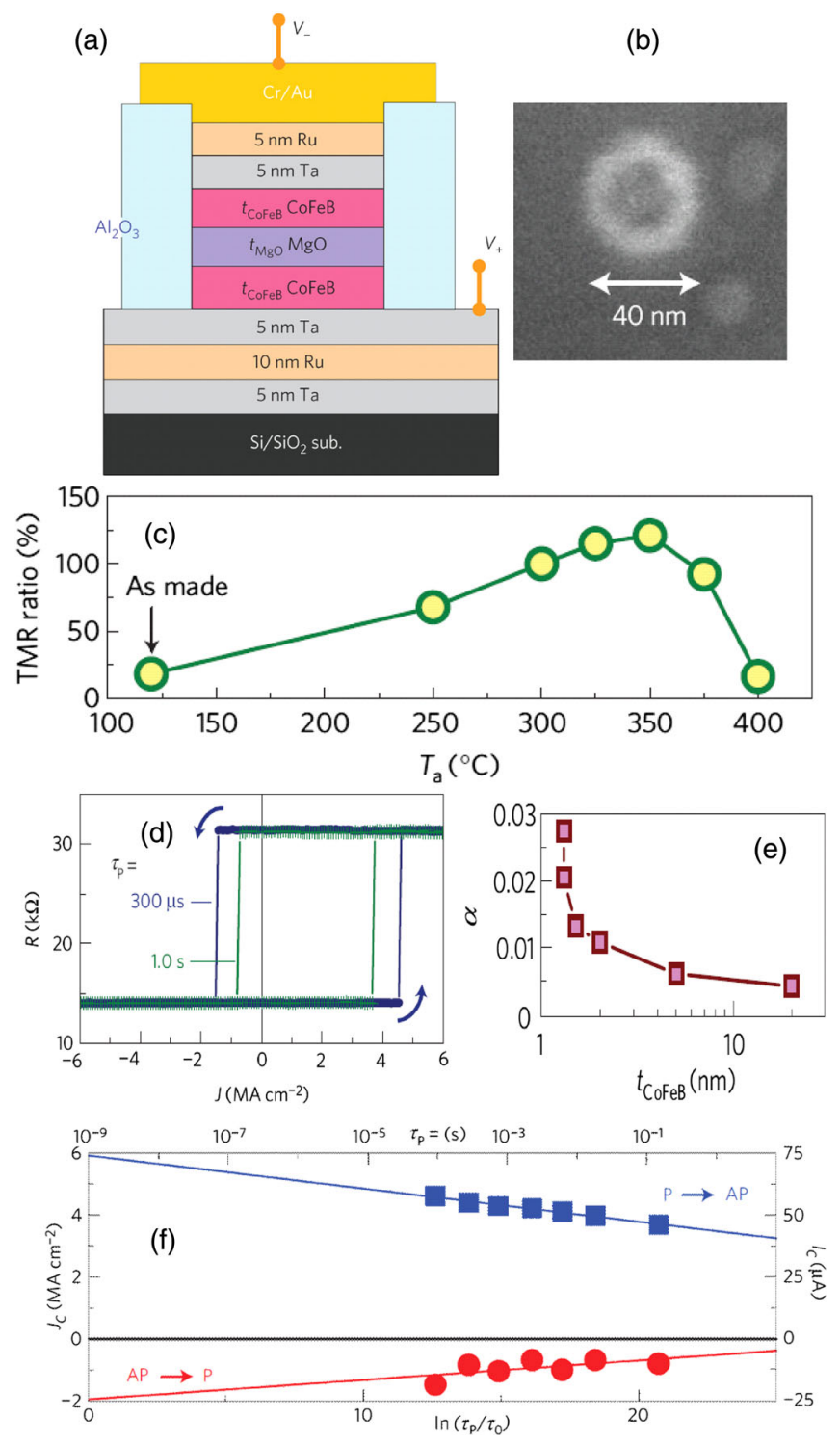

FIG. 12. Ta/CoFeB/MgO-based out-of-plane magnetized MTJ. (a) Schematic representation of an MTJ device used to study TMR and spin-transfer-torque switching. (b) Top view of an MTJ pillar by scanning electron microscopy. (c) TMR amplitude plotted against annealing temperature. (d) Example of spin-transfer-torque switching experiments performed with current pulse widths of $1.0 \mathrm{~s}$ and $300 \mu \mathrm{s}$. (e) Variation of the Gilbert damping coefficient as a function of $\mathrm{CoFeB}$ thickness, determined from ferromagnetic resonance (FMR) experiments. (f) Critical current density for STT switching as a function of $\ln \left(\tau_{P} / \tau_{0}\right), \tau_{P}=$ pulse width, $\tau_{0}=$ attempt time $\sim 1 \mathrm{~ns}$, from which $E=k_{B} T$ can be determined. From Ikeda et al., 2010.

measuring 40 or $150 \mathrm{~nm}$ in diameter [Fig. 12(b)]. Figure 12(c) shows that after annealing at $350{ }^{\circ} \mathrm{C}$ a TMR of up to $120 \%$ was obtained. This increase in TMR amplitude between the asdeposited state and after optimal annealing (here $350{ }^{\circ} \mathrm{C}$ ) is due to the improved crystallinity of the bcc $\mathrm{MgO}$ barrier and to the recrystallization of the $\mathrm{CoFeB}$ electrodes into bcc $\mathrm{CoFe}$ as the $\mathrm{B}$ is absorbed into the Ta buffer and capping layers. This improvement in the crystallinity of the magnetic electrodes and of the tunnel barrier yields the spin-filtering mechanism through which the giant tunnel magnetoresistance of crystalline MgO-based MTJs is generated (Butler et al., 2001; Parkin et al., 2004; Yuasa et al., 2004; Yuasa and Djayaprawira, 2007). The degradation of the TMR observed above $350{ }^{\circ} \mathrm{C}$ is ascribed to diffusion of the Ta through the $\mathrm{CoFeB}$ layers toward the $\mathrm{MgO}$ barrier (Ikeda et al., 2008).

In these samples, thanks to the strong interfacial anisotropy at the $\mathrm{CoFe}(\mathrm{B}) / \mathrm{MgO}$ interface, the magnetization of the $\mathrm{CoFeB}$ is pulled out of plane only when the $\mathrm{CoFeB}$ layers are thin enough to allow the iPMA to overcome the easy-plane demagnetizing energy. In this study, samples with $\mathrm{CoFeB}$ thicknesses of less than $1.3 \mathrm{~nm}$ could be magnetized out of plane after annealing at $300^{\circ} \mathrm{C}$.

Figure 12(d) shows typical hysteresis loops obtained in STT switching experiments. Switching occurs at relatively low current densities of around (2-4) $\times 10^{6} \mathrm{~A} / \mathrm{cm}^{2}$, indicating that the Gilbert damping in these systems is relatively weak compared to that measured for $\mathrm{Co} / \mathrm{Pt}$-based multilayers. Indeed, in STT experiments, the critical current for switching is proportional to the Gilbert damping (Slonczewski, 1996; Sun, 2000; see Sec. IV for further details). In out-of-plane magnetized samples, the critical current for switching in the macrospin model was determined (Sun, 2000) and is given by

$$
I_{c}^{\text {perp }}=\left(\frac{4 e}{\hbar}\right) \frac{\alpha k_{B} T}{g p} \Delta,
$$

where $\alpha$ is the Gilbert damping constant, $e$ is the electron charge, $\hbar$ is the Planck constant, $k_{B}$ is the Boltzmann constant, $T$ is the temperature, $p$ is the spin polarization, $\Delta$ is the thermal stability factor $=\Delta E / k_{B} T=K_{\text {eff }} V / k_{B} T$, and $g$ is a function of the spin polarization of the tunnel current and of the angle between the magnetizations of the free and reference layers (Berger, 1996, 1999; Slonczewski, 1996, 1999).

The Gilbert damping constant $\alpha$ was measured by ferromagnetic resonance (FMR) experiments [Fig. 12(e)] and found to significantly increase at low $\mathrm{CoFeB}$ thicknesses, in particular, within the range where perpendicular anisotropy is observed (below $1.3 \mathrm{~nm}$ ). This phenomenon is associated with diffusion of $\mathrm{Ta}$ into the $\mathrm{CoFeB}$ layer, reduction in the ordering temperature of the CoFeB layer as it thins, and with a spin-pumping effect (Kato et al., 2012; Shaw, Nembach, and Silva, 2012). In terms of STT-MRAM applications (see Sec. IV), this increase in damping at low thicknesses means the critical current for STT switching will increase. This increase is a drawback, but it can be limited or even avoided through material optimization, as seen in Sec. IV.

The thermal stability factor $\Delta$ was determined from the variations in the critical current for switching versus write current pulse duration [Fig. 12(f)] in the thermally activated regime (for current pulses longer than $\sim 10 \mathrm{~ns}$ ) using

$$
I_{c}=I_{c 0}\left\{1-\frac{k_{B} T}{\Delta E} \ln \left(\frac{\tau}{\tau_{0}}\right)\right\}
$$

(Koch, Katine, and Sun, 2004; Li and Zhang, 2004). A thermal stability factor of 43 was obtained for a $40 \mathrm{~nm}$ diameter MTJ, which corresponds to a retention time of $\sim 140 \mathrm{yr}$ for a single MTJ cell. A thermal stability factor above 
70 is required for a 1-Gbit chip and a probability of one failure over time of $10^{-4}$.

At about the same period as Ikeda et al. made their observations, Worledge (2010) and Worledge et al. (2011, 2014) from IBM also observed a strong perpendicular anisotropy of interfacial origin in $\mathrm{Ta} / \mathrm{CoFeB} / \mathrm{MgO}$-based MTJs. Worledge et al. investigated the influence of various buffer layers (Cr, Ta, Ru, V, Ti, $\mathrm{Al}, \mathrm{Mg}, \mathrm{Ru}, \mathrm{W}$ ) on the perpendicular anisotropy of buffer $/ \mathrm{Co}_{60} \mathrm{Fe}_{20} \mathrm{~B}_{20} \quad t / \mathrm{MgO}$ $0.9 \mathrm{~nm} / \mathrm{Fe} 0.3 \mathrm{~nm} / 5 \mathrm{TaN}$ in samples with $t$ ranging from 0.3 to $1.6 \mathrm{~nm}$. The samples were annealed for $1 \mathrm{~h}$ at $240^{\circ} \mathrm{C}$. Results indicated that the perpendicular anisotropy of the $\mathrm{CoFeB}$ layer was particularly strong with a Ta buffer layer. Thus, with $\mathrm{Ta} / \mathrm{CoFeB} / \mathrm{MgO}$, an overall interfacial anisotropy of $1.8 \mathrm{~mJ} / \mathrm{m}^{2}\left(1.8 \mathrm{erg} / \mathrm{cm}^{2}\right)$ was observed, whereas with $\mathrm{Ru} / \mathrm{CoFeB} / \mathrm{MgO}$, anisotropy was only $0.5 \mathrm{~mJ} / \mathrm{m}^{2}$. This difference is mainly due to better absorption of the $\mathrm{B}$ away from the $\mathrm{MgO}$ interface upon annealing when a Ta underlayer is present. Significant intermixing between $\mathrm{Ta}$ and $\mathrm{CoFeB}$ was also observed in these experiments, which is much less pronounced between $\mathrm{Ru}$ and $\mathrm{CoFeB}$.

All these results on $\mathrm{Ta} / \mathrm{CoFeB} / \mathrm{MgO}$-based $\mathrm{MTJ}$ were extremely encouraging for the development of out-of-plane magnetized STT-MRAM and numerous studies subsequently emerged describing developments of STT-MRAM cells attempting to achieve long enough retention and sufficiently low switching current.

\section{H. Interfacial PMA in epitaxial Fe/MgO-based systems}

iPMA at epitaxial $\mathrm{Fe} / \mathrm{MgO}$ interfaces has been investigated by several groups (Lee et al., 2011; Koo et al., 2013; Lambert et al., 2013; Koziol-Rachwal et al., 2014). Examining the sign and amplitude of the effective anisotropy by magnetometry and FMR experiments, Lambert et al. (2013) showed that Fe$\mathrm{MgO}$ interfaces possess a strong iPMA of $1.0 \pm 0.1 \mathrm{~mJ} / \mathrm{m}^{2}$ in fully epitaxial heterostructures composed of $\mathrm{MgO}$ (100) substrate/V, $10 \mathrm{~nm} / \mathrm{Fe} / \mathrm{MgO}, 1.2 \mathrm{~nm}$ (001) or $\mathrm{MgO}$ (100) substrate/Cr, $10 \mathrm{~nm} / \mathrm{Fe} / \mathrm{MgO}, 1.2 \mathrm{~nm}$ (001). As in sputtered samples, reorientation of the anisotropy from in plane to out of plane was observed below a certain critical thickness of the $\mathrm{Fe}$ MLs (6 nm for $\mathrm{V} / \mathrm{Fe} / \mathrm{MgO}$ and $4 \mathrm{~nm}$ for $\mathrm{Cr} / \mathrm{Fe} / \mathrm{MgO}$ ). Magnetometry experiments revealed the presence of 2-nm magnetic dead layers in Fe MLs on V that do not exist on $\mathrm{Cr}$. For a given $\mathrm{Fe}$ thickness, the demagnetizing energy is thus smaller in $\mathrm{V} / \mathrm{Fe} / \mathrm{MgO}$ than in $\mathrm{Cr} / \mathrm{Fe} / \mathrm{MgO}$. When this difference is taken into account, the interfacial anisotropy at the $\mathrm{Fe} / \mathrm{MgO}$ interface is found to be the same in the two series of samples $\left(\sim 1 \mathrm{~mJ} / \mathrm{m}^{2}\right)$. Similar magnetic dead layers were reported by various groups at sputtered $\mathrm{Ta} / \mathrm{CoFeB}$ interfaces (Ikeda et al., 2010; Worledge et al., 2011; Cuchet et al., 2014). This measured interfacial PMA was weaker than that measured by Koo et al. (2013) in $\mathrm{Cr} / \mathrm{Fe} / \mathrm{MgO}$ after annealing at $400{ }^{\circ} \mathrm{C}: K_{s} \sim 2 \mathrm{~mJ} / \mathrm{m}^{2}$. Koo et al. (2013) observed a strong increase in the interfacial PMA occurring between the $350{ }^{\circ} \mathrm{C}$ anneal and the $400{ }^{\circ} \mathrm{C}$ anneal that they ascribed to a variation of the interface composition due to oxygen atoms floating up from the $\mathrm{Cr}$ buffer layer and reaching the $\mathrm{MgO}$ interface. This hypothesis is consistent with earlier experiments from SPINTEC (Monso et al., 2002; Rodmacq et al., 2003, 2009; Manchon et al., 2008) which demonstrated that the PMA reaches a maximum in conditions where the $\mathrm{Co}(\mathrm{Fe}) / \mathrm{MO}$ interface is optimally oxidized $(\mathrm{MO}=$ oxide layer, i.e., $\mathrm{MgO}$ ) so as to allow the formation of $\mathrm{Co}(\mathrm{Fe})-\mathrm{O}-\mathrm{M}$ interfacial bonds.

Koziol-Rachwal et al. (2014) also demonstrated that PMA could be obtained in $(\mathrm{Fe} / \mathrm{MgO})$ multilayers with large numbers of repeats on the condition that the Fe layers are thin enough to allow the interfacial PMA at $\mathrm{Fe} / \mathrm{MgO}$ interfaces to overcome the demagnetizing energy. In Koziol-Rachwal et al. (2014) samples were epitaxially grown on an $\mathrm{MgO}$ (001) substrate and characterized by conversion electron Mössbauer spectroscopy (CEMS) and MOKE. In specific multilayers, $(\mathrm{Fe} 4 \mathrm{ML} / \mathrm{MgO} 3 \mathrm{ML})_{N}$, PMA was observed with an increased amplitude when the number of repeats $N$ was varied from 10 to 20 . The CEMS measurements with $57 \mathrm{Fe}$ ML probes located at different sublayers revealed that the PMA was produced in the inner part of the multilayered stack, whereas in the outer parts (top and bottom of the stacks), the magnetization was mostly in plane. Correlations between MOKE and CEMS experiments suggested a complex vortexlike magnetization distribution in the cross section of the stack. This was confirmed by micromagnetic simulations.

Ding and Poon (2012) also observed PMA in epitaxially grown sandwiches composed of substrate $\mathrm{MgO}(001) /$ $\mathrm{Co}_{20} \mathrm{Fe}_{50} \mathrm{Ge}_{30} / \mathrm{MgO} 5 \mathrm{~nm}$. The samples were grown by sputtering and annealed at $250{ }^{\circ} \mathrm{C}$ for $2 \mathrm{~h}$. Ding and Poon (2012) introduced $\mathrm{Ge}$ into the $\mathrm{CoFe}$ so as to obtain a Heusler B2 phase characterized by high spin polarization and significantly lower damping than in $\mathrm{CoFeB}$, as low as 0.0025 in bulk form (H. Lee et al., 2009; Wang et al., 2009); see Fig. 12(e). In these experiments, the magnetization of the CoFeGe layer was observed to point out of plane for CoFeGe thickness $t_{\mathrm{CoFeGe}}$ below $1.3 \mathrm{~nm}$. The PMA was mainly interfacial in origin, and an iPMA of $\sim 0.9 \mathrm{~mJ} / \mathrm{m}^{2}$ was determined by extrapolation of the linear variation of $K_{\text {eff }} t_{\mathrm{CoFeGe}} \mathrm{vs} t_{\mathrm{CoFeGe}}$ to zero thickness. Similar findings have been reported for Heulser alloy based $\mathrm{Ta} / \mathrm{Co}_{2} \mathrm{FeAl} / \mathrm{MgO}$ multilayers (Gabor et al., 2013).

\section{Gilbert damping measurements in $\mathrm{CoFeB} / \mathrm{MgO}$-based MTJ}

The Gilbert damping in $\mathrm{Ta} / \mathrm{CoFeB} / \mathrm{MgO}$-based MTJ is a determinant parameter in spin-transfer-torque magnetization switching and has been studied by various groups (Ikeda et al., 2010; Liu et al., 2011; Devolder, Ducrot et al., 2013; Iihama et al., 2014).

Devolder et al. measured the anisotropy, the Landé $g$ factor, and the Gilbert damping of series of $\mathrm{Ta} / \mathrm{Co}_{x} \mathrm{Fe}_{80-x} \mathrm{~B}_{20}$ $1 \mathrm{~nm} / \mathrm{MgO}$ ultrathin films displaying PMA. They varied the $\mathrm{CoFeB}$ composition and annealing conditions. Measurements were performed by applying a vector network analyzer ferromagnetic resonance technique (Devolder, Barisic et al., 2013). Their results showed that, in Fe-rich FeCoB alloys, the PMA increases quite significantly upon crystallization, whereas the damping $\alpha$ only increases slightly [e.g., in amorphous- $\mathrm{Co}_{20} \mathrm{Fe}_{60} \mathrm{~B}_{20} 1 \mathrm{~nm}, \alpha=0.014 \pm 0.002$, $\mu_{0}\left(H_{K}-M_{s}\right)=45 \pm 10 \mathrm{mT}$; in crystalline- $\mathrm{Co}_{20} \mathrm{Fe}_{60} \mathrm{~B}_{20}$ $\left.1 \mathrm{~nm}, \quad \alpha=0.015 \pm 0.002, \mu_{0}\left(H_{K}-M_{s}\right)=430 \pm 10 \mathrm{mT}\right]$. When cobalt content was high, the iPMA also increased 
upon crystallization of the $\mathrm{CoFeB}$, but less than in Fe-rich alloys. However, the effective anisotropy was observed to change sign from negative (in plane) to positive (out of plane) as the damping decreased [e.g., in amorphous- $\mathrm{Co}_{60} \mathrm{Fe}_{20} \mathrm{~B}_{20}$ $1 \mathrm{~nm}, \quad \alpha=0.016 \pm 0.002, \quad \mu_{0}\left(H_{K}-M_{s}\right)=-80 \pm 10 \mathrm{mT}$, whereas in crystalline- $\mathrm{Co}_{60} \mathrm{Fe}_{20} \mathrm{~B}_{20} 1 \mathrm{~nm}, \alpha=0.016 \pm 0.002$, $\left.\mu_{0}\left(H_{K}-M_{s}\right)=82 \pm 10 \mathrm{mT}\right]$. Furthermore, by analyzing their own results alongside other published data, they found a correlation between the Gilbert damping and $(g-2)^{2}$, as expected in transition metals (Kambersky, 1976).

Iihama et al. (2014) investigated the magnetization dynamics of both $\mathrm{Ta} / \mathrm{CoFeB} / \mathrm{MgO}$ and $\mathrm{Ta} / \mathrm{CoFeB} / \mathrm{Ta}$ films using an all-optical pump-probe method. The dependence of the precession frequency and the relaxation time on the field strength and direction applied could readily be explained by the Landau-Lifshitz-Gilbert equation when the magnetic anisotropy distribution in the film was taken into account. The Gilbert damping values were found to increase linearly with increasing inverse $\mathrm{CoFeB}$ thickness [see Fig. 13(a)] following an $\alpha=\alpha_{\text {bulk }}+\alpha_{s} / t_{\mathrm{CoFeB}}$ law, consistent with the earlier observations by Ikeda et al. (2010) [see Fig. 12(e)] and similar to the variations observed in $\mathrm{Co} / \mathrm{Pd}$ multilayers (Barati et al., 2013). This variation can be explained by a spinpumping effect (Tserkovnyak, Brataas, and Bauer, 2002). The slope of the $\alpha$ vs $1 / t_{\mathrm{CoFeB}}$ for $\mathrm{Ta} / \mathrm{CoFeB} / \mathrm{MgO}$ films is smaller than that for $\mathrm{Ta} / \mathrm{CoFeB} / \mathrm{Ta}$ films, suggesting that the enhancement of $\alpha$ is caused by the Ta/CoFeB interface. Comparison of the dependence of $\alpha$ and the PMA constant $K_{u}$ on the annealing temperature revealed no correlation between $\alpha$ and $K_{u}$ [see Fig. 13(b)]. Indeed, $K_{u}$ shows a pronounced maximum after annealing at about $300{ }^{\circ} \mathrm{C}$ [consistent with the data reported in Fig. 10(a)], while the damping is relatively unchanged up to $300^{\circ} \mathrm{C}$. Above this annealing temperature, a significant diffusion of $\mathrm{Ta}$ into the $\mathrm{CoFeB}$ is expected which could explain the increase of $\alpha$ above $300{ }^{\circ} \mathrm{C}$.

As explained previously, this tendency of damping to increase at low CoFeB thicknesses is a drawback in the context of STT magnetization switching, in particular, for STT-MRAM. However, we see in Sec. IV that its extent can be
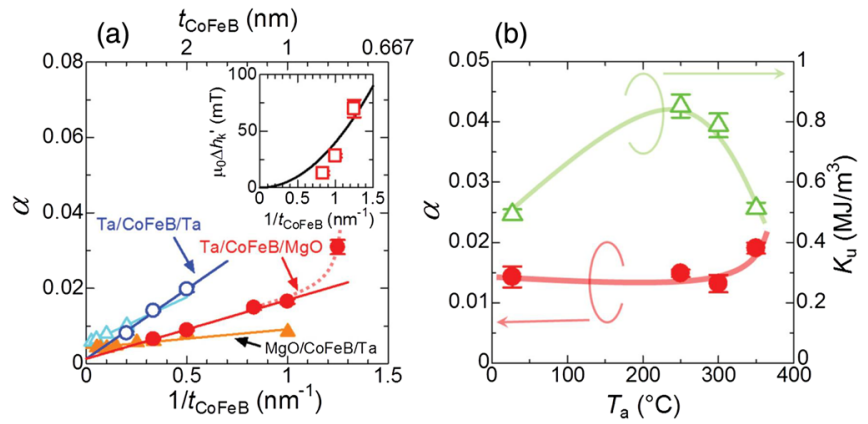

FIG. 13. (a) Gilbert damping constant $\alpha$ vs $1 / t_{\mathrm{CoFeB}}$ for films composed of $\mathrm{Ta} / \mathrm{CoFeB} / \mathrm{MgO}$ (solid circles) and $\mathrm{Ta} / \mathrm{CoFeB} / \mathrm{Ta}$ (open circles). The solid lines indicate linear relationships; the broken line is a visual guide. The inset represents the rms of the PMA field obtained by fitting the damping rate of the oscillatory magnetization dynamics measured by MOKE plotted against $1 / t_{\mathrm{CoFeB}}$. (b) Damping $\alpha$ and anisotropy $K_{u}$ as a function of the annealing temperature $T_{a}$ in $\mathrm{Ta} / \mathrm{CoFeB} 1.2 \mathrm{~nm} / \mathrm{MgO}$. The solid lines are visual guides. From Iihama et al., 2014. significantly reduced by optimizing the stack composition, in particular, by sandwiching the storage $\mathrm{CoFeB}$ layer between two $\mathrm{MgO}$ layers.

\section{PHYSICAL ORIGIN OF THE INTERFACIAL PMA}

This section provides a theoretical overview of the physical mechanisms generating iPMA at transition metal/oxide interfaces. We mainly focused on $(\mathrm{Co}) \mathrm{Fe} / \mathrm{MgO}$ interfaces and MTJs since these structures are central to spintronics applications, as we have seen in the two previous sections. To begin with, let us consider a FM thin film placed between two interfacial regions which could be nonmagnetic metal (NM), vacuum, or insulator (Fig. 14). The case of FM/NM metallic interfaces (Chappert and Bruno, 1988; Daalderop, Kelly, and Schuurmans, 1990; Daalderop, Kelly, and den Broeder, 1992; Kyuno, Yamamoto, and Asano, 1992; Wang, Wu, and Freeman, 1993a; Johnson et al., 1995, 1996) will be omitted as it is out of scope for this review. We therefore focus on FM/vacuum and FM/oxide interfaces and MTJs based on these structures.

The effective anisotropy for this type of FM thin film can be written as

$$
K_{\text {eff }}=K_{V}+K_{d}+\frac{K_{s 1}+K_{s 2}}{t},
$$

where the first two terms represent magnetocrystalline $\left(K_{V}\right)$ and demagnetizing $\left(K_{d}<0\right)$ volume contributions and the last term accounts for anisotropic energies per unit area from the two interfaces $\left(K_{s 1}\right.$ and $\left.K_{s 2}\right) ; t$ is the FM film thickness [cf. Eq. (8)]. The demagnetization term $K_{d}$ is due to dipole-dipole interactions and in the case of $3 d$ transition metals can be written as

$K_{d}=\frac{\mu_{0}}{4 \pi V} \sum_{i \neq j} \frac{1}{\mathbf{r}_{i j}^{3}}\left(\mathbf{m}_{i} \cdot \mathbf{m}_{j}-3 \frac{\left(\mathbf{r}_{i j} \cdot \mathbf{m}_{i}\right)\left(\mathbf{r}_{i j} \cdot \mathbf{m}_{j}\right)}{\mathbf{r}_{i j}^{2}}\right)$

(Jansen, 1988; Bruno, 1993), where $\mathbf{m}_{i}$ represents the dipolar magnetic moment of atom $i, \mathbf{r}_{i j}$ is a vector from atom $i$ to $j, \mu_{0}$ is the vacuum permeability, and $V$ is the thin film volume. For $3 d$ infinite FM materials the sum of the dipole-dipole energies cancels out in symmetric crystals including $\mathrm{Fe}, \mathrm{Ni}$ cubic, and Co hcp with low $c / a$ ratio deviation (Bloch and Gentile, 1931;

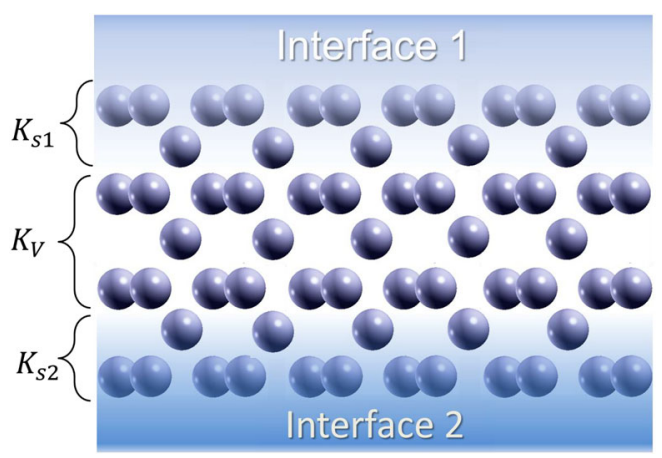

FIG. 14. Schematic representation of the contributions from interface and volume anisotropy in thin films. Interfacial regions 1 and 2 may be vacuum or oxide. 
Daalderop, Kelly, and Schuurmans, 1990; Bruno, 1993). However, in finite size samples, this dipolar interaction energy calculated using Eq. (14) does not fully vanish and results in the shape anisotropy (which favors in-plane magnetization with thin films) [cf. Eq. (7)]. The other terms in Eq. (13), $K_{V}$ and $K_{s 1(2)}$, are essentially derived from the spin-orbit coupling (Bruno, 1993). Since the volume magnetocrystalline anisotropy contribution $K_{V}$ has already been intensively studied (Daalderop, Kelly, and Schuurmans, 1990, 1994a; Bruno, 1993), we focused here on the interfacial contribution $K_{s 1(2)}$ arising at the interface between the insulating oxide and the FM metal. We first briefly introduce essential grounds for spin-orbit interaction which cause electronic orbital hybridization. We then overview the evolution of the understanding of interfacial anisotropy mechanisms in an historical perspective starting from the case of ultrathin films and monolayers toward the case of FM/oxide interfaces focusing, in particular, on the model case of $\mathrm{Fe} / \mathrm{MgO}$ structures and impact of interfacial stoichiometry on the interfacial anisotropy.

\section{A. Qualitative description of anisotropy based on electronic hybridization}

The spin-orbit interaction links the electron spin to the magnetic field created by its orbital motion around the nucleus. When the symmetric potential is spherical, $V(r)$ is described by the following Hamiltonian term (Jones and March, 1973; Bruno, 1993):

$H_{\mathrm{so}}=\frac{e \hbar}{4 m^{2} c^{2} r} \frac{d V}{d r} \boldsymbol{\sigma} \cdot(\mathbf{r} \times \mathbf{p})=\frac{e \hbar}{2 m^{2} c^{2} r} \frac{d V}{d r} \mathbf{L} \cdot \mathbf{S}=\xi(r) \mathbf{L} \cdot \mathbf{S}$,

where the product of the orbital angular momentum and spin operators can be written in turn using the longitudinal (i.e., along the quantization axis $z$ ) and ladder operators as

$$
\mathbf{L} \cdot \mathbf{S}=\frac{1}{2}\left(L_{+} S_{-}+L_{-} S_{+}\right)+L_{z} S_{z} .
$$

Using the properties of the spin operator, the Hamiltonian (15) can be rewritten in matrix form, thus

$$
H_{\mathrm{so}}=\xi(r)\left(\begin{array}{cc}
H_{\mathrm{So}}^{\uparrow \uparrow} & H_{\mathrm{So}}^{\uparrow \downarrow} \\
H_{\mathrm{SO}}^{\downarrow \uparrow} & H_{\mathrm{So}}^{\downarrow \downarrow}
\end{array}\right)=\frac{1}{2} \xi(r)\left(\begin{array}{cc}
L_{z} & L_{-} \\
L_{+} & -L_{z}
\end{array}\right),
$$

where orbital momentum operator components in Eq. (16) are square matrices of size $(2 l+1)$. To visualize the effect of spinorbit coupling on orbital angular and spin moments, we first consider the eigenvalues and eigenstates of the spin-orbit Hamiltonian (15) in the case of a free magnetic atom with $l=2 d$ orbitals. In the absence of the spin-orbit interaction, the system is characterized by ten eigenstates, i.e., five eigenstates for the $L_{z}$ operator for each spin. These eigenstates represent complex spherical harmonics $Y_{2}^{m}(\theta, \varphi)$, which we denote as $|m ; \uparrow\rangle$ and $|m ; \downarrow\rangle$ using the integer magnetic quantum number $m \in[-2 ; 2]$. The Hamiltonian matrix in these conditions will be diagonal in the spin space. When the spin-orbit interaction is switched on, six and four eigenstates appear corresponding to a total angular momentum of 5/2 and
$3 / 2$, respectively. Using Clebsch-Gordan coefficients, these eigenstates can be written as

$$
\begin{aligned}
\left|\frac{5}{2} ; \frac{5}{2}\right\rangle & =|2 ; \uparrow\rangle, \\
\left|\frac{5}{2} ; \frac{3}{2}\right\rangle & =\sqrt{\frac{4}{5}}|1 ; \uparrow\rangle+\sqrt{\frac{1}{5}}|2 ; \downarrow\rangle, \\
\left|\frac{5}{2} ; \frac{1}{2}\right\rangle & =\sqrt{\frac{3}{5}}|0 ; \uparrow\rangle+\sqrt{\frac{2}{5}}|1 ; \downarrow\rangle, \\
\left|\frac{5}{2} ;-\frac{1}{2}\right\rangle & =\sqrt{\frac{2}{5}}|-1 ; \uparrow\rangle+\sqrt{\frac{3}{5}}|0 ; \downarrow\rangle, \\
\left|\frac{5}{2} ;-\frac{3}{2}\right\rangle & =\sqrt{\frac{1}{5}}|-2 ; \uparrow\rangle+\sqrt{\frac{4}{5}}|-1 ; \downarrow\rangle, \\
\left|\frac{5}{2} ;-\frac{5}{2}\right\rangle & =|-2 ; \downarrow\rangle, \\
\left|\frac{3}{2} ; \frac{3}{2}\right\rangle & =-\sqrt{\frac{1}{5}}|1 ; \uparrow\rangle+\sqrt{\frac{4}{5}}|2 ; \downarrow\rangle, \\
\left|\frac{3}{2} ; \frac{1}{2}\right\rangle & =-\sqrt{\frac{2}{5}}|0 ; \uparrow\rangle+\sqrt{\frac{3}{5}}|1 ; \downarrow\rangle, \\
\left|\frac{3}{2} ;-\frac{1}{2}\right\rangle & =-\sqrt{\frac{3}{5}}|-1 ; \uparrow\rangle+\sqrt{\frac{2}{5}}|0 ; \downarrow\rangle, \\
\left|\frac{3}{2} ;-\frac{3}{2}\right\rangle & =-\sqrt{\frac{4}{5}}|-2 ; \uparrow\rangle+\sqrt{\frac{1}{5}}|-1 ; \downarrow\rangle .
\end{aligned}
$$

This notation reveals how the spin-orbit interaction couples within the same states spin and angular momenta. However, it is more convenient to pass from complex spherical harmonics to their related real ones as follows:

$$
\begin{aligned}
& \left|d_{x y} ; \sigma\right\rangle=\frac{i}{\sqrt{2}}(|-2 ; \sigma\rangle-|2 ; \sigma\rangle), \\
& \left|d_{y z} ; \sigma\right\rangle=\frac{i}{\sqrt{2}}(|-1 ; \sigma\rangle+|1 ; \sigma\rangle), \\
& \left|d_{z^{2}} ; \sigma\right\rangle=|0 ; \sigma\rangle, \\
& \left|d_{x z} ; \sigma\right\rangle=\frac{1}{\sqrt{2}}(|-1 ; \sigma\rangle-|1 ; \sigma\rangle), \\
& \left|d_{x^{2}} ; \sigma\right\rangle=\frac{1}{\sqrt{2}}(|-2 ; \sigma\rangle+|2 ; \sigma\rangle),
\end{aligned}
$$

where, for simplicity, $z^{2}$ and $x^{2}$ are used to indicate the orbital characters $3 z^{2}-r^{2}$ and $x^{2}-y^{2}$. For example, from Eqs. (18) and (19), it becomes obvious that the spin-up $d_{z}{ }^{2}$ state is mixed with the spin-down $d_{x z(y z)}$ states due to the spin-orbit interactions in states with $m_{j}= \pm 1 / 2$. This case will be of particular interest to us when we consider the origins of PMA at $\mathrm{Fe} / \mathrm{MgO}$ interfaces (see later). In fact, the real spherical harmonics given by Eq. (19) represent an appropriate choice to describe $3 d$ magnetic metals where the spherical symmetry is broken due to the presence of a crystal field which is strong enough to quench the orbital moment (Bloch and Gentile, 1931; Brooks, 1940).

Using these atomic real spherical harmonics as a basic set of Bloch states for bcc Fe in the framework of a tight-binding approach, Abate and Adsente (1965) investigated the impact of spin-orbit coupling on the band structure, assuming that the matrix elements of the spin-orbit Hamiltonian (15) between the Bloch functions of the crystal reduce to those based on 
atomic functions expressed in terms of real spherical harmonics given by Eq. (19). With a natural choice of quantization axis along the magnetization direction characterized by the Euler angles $\theta$ and $\varphi$, the matrix elements of $\mathbf{L} \cdot \mathbf{S}$ between $d$ orbitals are given in Table I.

Abate and Adsente (1965) indicated that for bcc Fe, the spin-orbit coupling weakly connects the electronic spins with the crystal field giving rise to magnetic anisotropy. They also noticed that this spin-orbit interaction eliminates some degeneracy in the central part of the Brillouin zone, including along the $\Gamma-H$ line where the Bloch states are represented with $\Delta$ symmetry. These regions are particularly important for transport and anisotropy at $\mathrm{Fe} / \mathrm{MgO}$ interfaces and MTJs, as we will see.

Using a similar approach to Abate et al., Takayama, Bohnen, and Fulde (1976) calculated the magnetic surface anisotropy for transition metals, paying particular attention to the (001) surface of $\mathrm{Ni}$, including the case of the isolated monolayer, and found values up to $-0.2 \mathrm{erg} / \mathrm{cm}^{2}$. For $\mathrm{Co}, \mathrm{Fe}$, and Ni monolayers, Bruno and Renard (1989) predicted a surface anisotropy of around $-1 \mathrm{erg} / \mathrm{cm}^{2}$. This surface anisotropy strongly depends on the crystal field parameters for the material and on the filling of the $3 d$ bands. Importantly, Bruno (1989), based on a perturbative theory, outlined the close connection between magnetocrystalline and orbital moment anisotropies (of the order of $0.1 \mu_{B}$ per atom) in itinerant ferromagnetic monolayers. The importance of the crystal field energy and of the filling of the valence band was also emphasized. van der Laan (1998) extended Bruno's model by including the majority spin band orbital moment and spin flip excitations. For anisotropy energy, the second order perturbation theory gives

$$
\Delta E_{\text {so }}=K_{0}+K_{1} \sin ^{2} \theta
$$

The virtue of perturbation theory is that it can be used to find the anisotropy constants without explicitly calculating the system's total energy as a function of magnetization direction. However, it has the disadvantage that it cannot handle degenerate levels and Fermi surface deformations correctly (Bruno, 1993; Lessard, Moos, and Hübner, 1997; Yang, Savrasov, and Kotliar, 2001). In all these works, the magnetic monolayers were found to display in-plane anisotropy. However, Gay and Richter (1986) reported PMA for Fe and V monolayers and IMA for Ni. For instance, the anisotropy of the Fe monolayer was found to be $\sim 0.4 \mathrm{meV} /$ atom, which is 2 or 3 orders of magnitude the anisotropy of bulk Fe because of the reduced symmetry at the surface which allows the anisotropy to appear in second order (Gay and Richter, 1986). Daalderop, Kelly, and Schuurmans (1994a) evaluated magnetic anisotropy from first principles for a freestanding Co monolayer and found it to oscillate between IMA and PMA as a function of band filling. They went into great detail on the mechanisms through which orbital hybridization affects anisotropy due to spin-orbit

TABLE I. Matrix elements of the $\mathbf{L} \cdot \mathbf{S}$ part of the spin-orbit Hamiltonian between $d$ orbitals.

\begin{tabular}{|c|c|c|c|c|c|c|c|c|c|c|}
\hline & $\left|d_{x y} ; \uparrow\right\rangle$ & $\left|d_{y z} ; \uparrow\right\rangle$ & $\left|d_{x z} ; \uparrow\right\rangle$ & $\left|d_{z^{2}} ; \uparrow\right\rangle$ & $\left|d_{x^{2}} ; \uparrow\right\rangle$ & $\left|d_{x y} ; \downarrow\right\rangle$ & $\left|d_{y z} ; \downarrow\right\rangle$ & $\left|d_{x z} ; \downarrow\right\rangle$ & $\left|d_{z^{2}} ; \downarrow\right\rangle$ & $\left|d_{x^{2}} ; \downarrow\right\rangle$ \\
\hline$\left\langle d_{x y} ; \uparrow\right|$ & 0 & $\begin{array}{l}\frac{i}{2} \sin \theta \\
\sin \varphi\end{array}$ & $\begin{array}{r}-\frac{i}{2} \sin \theta \\
\cos \varphi\end{array}$ & 0 & $i \cos \theta$ & 0 & $\begin{array}{l}\frac{1}{2}(\cos \varphi \\
\quad+i \cos \theta \\
\quad \sin \varphi)\end{array}$ & $\begin{array}{c}\frac{1}{2}(\sin \varphi \\
-i \cos \theta \\
\cos \varphi)\end{array}$ & 0 & $-i \sin \theta$ \\
\hline$\left\langle d_{y z} ; \uparrow\right|$ & $\begin{array}{r}-\frac{i}{2} \sin \theta \\
\quad \sin \varphi\end{array}$ & 0 & $\frac{i}{2} \cos \theta$ & $\begin{array}{r}-\frac{i \sqrt{3}}{2} \sin \theta \\
\cos \varphi\end{array}$ & $\begin{array}{r}-\frac{i}{2} \sin \theta \\
\cos \varphi\end{array}$ & $\begin{array}{c}-\frac{1}{2}(\cos \varphi \\
+i \cos \theta \\
\sin \varphi)\end{array}$ & 0 & $-\frac{i}{2} \sin \theta$ & $\begin{array}{l}\frac{i \sqrt{3}}{2}(\sin \varphi \\
-i \cos \theta \\
\quad \cos \varphi)\end{array}$ & $\begin{array}{c}\frac{1}{2}(\sin \varphi \\
-i \cos \theta \\
\quad \cos \varphi)\end{array}$ \\
\hline$\left\langle d_{x z} ; \uparrow\right|$ & $\begin{array}{l}\frac{i}{2} \sin \theta \\
\cos \varphi\end{array}$ & $-\frac{i}{2} \cos \theta$ & 0 & $\begin{array}{r}\frac{i \sqrt{3}}{2} \sin \theta \\
\sin \varphi\end{array}$ & $\begin{array}{r}-\frac{i}{2} \sin \theta \\
\sin \varphi\end{array}$ & $\begin{array}{c}-\frac{1}{2}(\sin \varphi \\
-i \cos \theta \\
\cos \varphi)\end{array}$ & $\frac{i}{2} \sin \theta$ & 0 & $\begin{array}{l}\frac{\sqrt{3}}{2}(\cos \varphi \\
\quad+i \cos \theta \\
\quad \sin \varphi)\end{array}$ & $\begin{array}{c}-\frac{1}{2}(\cos \varphi \\
+i \cos \theta \\
\quad \sin \varphi)\end{array}$ \\
\hline$\left\langle d_{z^{2}} ; \uparrow\right|$ & 0 & $\begin{array}{r}\frac{i \sqrt{3}}{2} \sin \theta \\
\cos \varphi\end{array}$ & $\begin{array}{r}-\frac{i \sqrt{3}}{2} \sin \theta \\
\sin \varphi\end{array}$ & 0 & 0 & 0 & $\begin{array}{c}-\frac{\sqrt{3}}{2}(\sin \varphi \\
-i \cos \theta \\
\cos \varphi)\end{array}$ & $\begin{array}{c}-\frac{\sqrt{3}}{2}(\cos \varphi \\
+i \cos \theta \\
\sin \varphi)\end{array}$ & 0 & 0 \\
\hline$\left\langle d_{x^{2}} ; \uparrow\right|$ & $-i \cos \theta$ & $\begin{array}{l}\frac{i}{2} \sin \theta \\
\cos \varphi\end{array}$ & $\begin{array}{l}\frac{i}{2} \sin \theta \\
\sin \varphi\end{array}$ & 0 & 0 & $i \sin \theta$ & $\begin{array}{c}-\frac{1}{2}(\sin \varphi \\
-i \cos \theta \\
\cos \varphi)\end{array}$ & $\begin{array}{l}\frac{1}{2}(\cos \varphi \\
\quad+i \cos \theta \\
\quad \sin \varphi)\end{array}$ & 0 & 0 \\
\hline$\left\langle d_{x y} ; \downarrow\right|$ & 0 & $\begin{array}{c}-\frac{1}{2}(\cos \varphi \\
-i \cos \theta \\
\sin \varphi)\end{array}$ & $\begin{array}{c}-\frac{1}{2}(\sin \varphi \\
-i \cos \theta \\
\cos \varphi)\end{array}$ & 0 & $-i \sin \theta$ & 0 & $\begin{array}{r}-\frac{i}{2} \sin \theta \\
\sin \varphi\end{array}$ & $\begin{array}{l}\frac{i}{2} \sin \theta \\
\quad \cos \varphi\end{array}$ & 0 & $-i \cos \theta$ \\
\hline$\left\langle d_{y z} ; \downarrow\right|$ & $\begin{array}{c}\frac{1}{2}(\cos \varphi \\
-i \cos \theta \\
\sin \varphi)\end{array}$ & 0 & $-\frac{i}{2} \sin \theta$ & $\begin{array}{c}-\frac{\sqrt{3}}{2}(\sin \varphi \\
+i \cos \theta \\
\quad \cos \varphi)\end{array}$ & $\begin{array}{c}-\frac{1}{2}(\sin \varphi \\
+i \cos \theta \\
\quad \cos \varphi)\end{array}$ & $\begin{array}{l}\frac{i}{2} \sin \theta \\
\quad \sin \varphi\end{array}$ & 0 & $-\frac{i}{2} \cos \theta$ & $\begin{array}{r}\frac{i \sqrt{3}}{2} \sin \theta \\
\cos \varphi\end{array}$ & $\begin{array}{l}\frac{i}{2} \sin \theta \\
\cos \varphi\end{array}$ \\
\hline$\left\langle d_{x z} ; \downarrow\right|$ & $\begin{array}{l}\frac{1}{2}(\sin \varphi \\
\quad+i \cos \theta \\
\quad \cos \varphi)\end{array}$ & $\frac{i}{2} \sin \theta$ & 0 & $\begin{array}{c}\frac{\sqrt{3}}{2}(-\cos \varphi \\
\quad+i \cos \theta \\
\quad \sin \varphi)\end{array}$ & $\begin{array}{c}\frac{1}{2}(\cos \varphi \\
-i \cos \theta \\
\sin \varphi)\end{array}$ & $\begin{array}{r}-\frac{i}{2} \sin \theta \\
\cos \varphi\end{array}$ & $\frac{i}{2} \cos \theta$ & 0 & $\begin{array}{r}-\frac{i \sqrt{3}}{2} \sin \theta \\
\sin \varphi\end{array}$ & $\begin{array}{l}\frac{i}{2} \sin \theta \\
\quad \sin \varphi\end{array}$ \\
\hline$\left\langle d_{z^{2}} ; \downarrow\right|$ & 0 & $\begin{array}{l}\frac{\sqrt{3}}{2}(\sin \varphi \\
\quad+i \cos \theta \\
\quad \cos \varphi)\end{array}$ & $\begin{array}{c}\frac{\sqrt{3}}{2}(\cos \varphi \\
-i \cos \theta \\
\sin \varphi)\end{array}$ & 0 & 0 & 0 & $\begin{array}{r}-\frac{i \sqrt{3}}{2} \sin \theta \\
\cos \varphi\end{array}$ & $\begin{array}{r}\frac{i \sqrt{3}}{2} \sin \theta \\
\quad \sin \varphi\end{array}$ & 0 & 0 \\
\hline$\left\langle d_{x^{2}} ; \downarrow\right|$ & $i \sin \theta$ & $\begin{array}{l}\frac{1}{2}(\sin \varphi \\
+i \cos \theta \\
\quad \cos \varphi)\end{array}$ & $\begin{array}{c}-\frac{1}{2}(\cos \varphi \\
-i \cos \theta \\
\sin \varphi)\end{array}$ & 0 & 0 & $i \cos \theta$ & $\begin{array}{r}-\frac{i}{2} \sin \theta \\
\cos \varphi\end{array}$ & $\begin{array}{r}-\frac{i}{2} \sin \theta \\
\quad \sin \varphi\end{array}$ & 0 & 0 \\
\hline
\end{tabular}


coupling around high symmetry points. Higher order anisotropy contributions $K_{2}$ in the total anisotropy energy

$$
\Delta E_{\mathrm{so}}=K_{0}+K_{1} \sin ^{2} \theta+K_{2} \sin ^{4} \theta
$$

have also been estimated to be a few percent of $K_{1}$ at certain band fillings (Farle, 1998). Újfalussy et al. (1996) also reported IMA for a Co monolayer using the screened KKR (Korringa-Kohn-Rostoker) method. For further details on $a b$ initio methods applied for anisotropy studies in monolayers, see Wu and Freeman (1999).

It is interesting to note that the behavior of magnetic surface anisotropy can be explained in the context of Néel's theory (Néel, 1954). As demonstrated by Skomski (1998) in the framework of the tight-binding moments theorem, Néel's model goes far beyond the original assumption of quasidipolar pair interactions between localized atoms. In other words, it already contains the metallic band structure and the influence of interface atoms at a rudimentary level. Starting from the tight-binding Hamiltonian which includes the spin-orbit matrix elements given in Table I, Skomski showed that magnetic anisotropy arises in the third moment. By assuming that the $d_{x y}$ and $d_{x^{2}}$ subbands are wider than the $d_{z^{2}}, d_{y z}$, and $d_{x z}$ ones, he reported the $K_{1}$ dependence as a function of the number of electrons for a (001) square lattice monolayer such as that schematically represented in Fig. 15. This dependence reproduces the aforementioned oscillatory behavior of magnetic surface anisotropy as a function of band filling in agreement with the diatomic pair model (Wang, Wu, and Freeman, 1993b). The precise magnitude of the anisotropy depends on the number of nearest neighbors, their coordination, and interatomic distances. Indeed, the inclusion of higher order moments, corresponding to more distant neighbors (Skomski et al., 2010), has been shown to indicate that the Néel coupling constants become complicated functions of band filling and atomic neighborhood. Notably, in his pioneering work, Néel considered only nearest neighbors. In this case he predicted surface anisotropy values between 0.13 and $1.3 \mathrm{erg} / \mathrm{cm}^{2}$ for all surfaces of fcc, bcc, and simple cubic lattices except bcc (001), bcc (111), and simple cubic (111) cases. He pointed out, however, that considering secondnearest neighbors, which are especially important for bcc

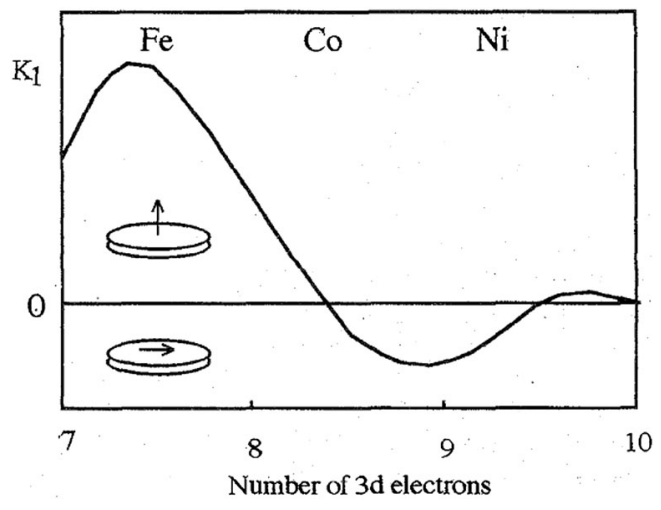

FIG. 15. Schematic band-filling dependence of the anisotropy. As a guide for the eye, approximate atomic meanings of the band filling are shown at the top of the figure. From Skomski, 1998. lattices, would also lead to the appearance of the surface anisotropy in those cases (Néel, 1954).

\section{B. $A b$ initio calculations, comparison with experimental data}

We now proceed to examine $(\mathrm{Co}) \mathrm{Fe} / \mathrm{MgO}$ interfaces using first-principles calculations where magnetocrystalline anisotropy energy (MAE) is calculated based on the difference in self-consistent total energies; alternatively it can be determined by the difference in the sum of the one-electron energies according to the force theorem (Weinert, Watson, and Davenport, 1985; Daalderop, Kelly, and Schuurmans, 1990). The latter method has been validated for surfaces and interfaces where the leading second-order part of MAE dominates in the spin orbit (Wang et al., 1996) and it is often used for transition metal interfaces (Blonski and Hafner, 2009).

A standard procedure to evaluate MAE from first principles comprises two steps. First, scalar-relativistic self-consistent spin-polarized calculations are performed in order to determine the magnetic ground state. After that the spin-orbit coupling is included and the total energy of the system is determined as a function of the magnetic moments direction. The MAE is then found as a difference of the total energy values for in-plane and out-of-plane orientation of the magnetization. As an optional step, structural relaxation in shape and/or volume can and should be performed for determining the most stable interfacial geometries. This procedure is usually carried out using density functional theory based approaches which differ by the methods used to resolve Kohn-Sham equations as a function of choice of approximations for Hamiltonian (e.g., choice of external and exchange correlation potentials) and basis sets for wave functions (e.g., plane waves, local orbitals). Among methods employed in recent reports for MCA calculations in transition metal/oxide interfaces (Nakamura et al., 2010; Niranjan et al., 2010; Yang et al., 2011; Khoo et al., 2013; Suzuki et al., 2013; Kanai et al., 2014; Zhang et al., 2014; Bose et al., 2016; Li, Barreteau, and Smogunov, 2016) are full potential linearized augmented plane wave, full potential linear combination of atomic orbitals, screened Kohn-KorringaRostocker (sKKR), projector-augmented wave (PAW), or ultrasoft (US) pseudopotentials and others based on localized orbitals or plane waves basis set. For instance, the latter represents a natural choice for systems with periodic boundary conditions and is used in PAW and/or US pseudopotentials based on the Vienna $a b$ initio simulation package (VASP) [see Blonski and Hafner (2009) and references therein] and the QUANTUM ESPRESSO (Giannozzi et al., 2009) package which are often used for efficient MCA calculations at transition metal/oxide interfaces. It is also interesting to point out that almost all these approaches qualitatively give identical results for MCA in these interfaces with slight quantitative variations of about $10 \%$ as analyzed, for instance, by Bose et al. (2016) for $\mathrm{Fe} / \mathrm{MgO}$ interfaces. Moreover, as discussed further the anisotropy values are in relatively good agreement with experiments as well. In particular, this can be explained by the fact that unlike the bulk MCA which is of the order of $\mu \mathrm{eV}$ and therefore requires tremendous convergence efforts, the surface and interfacial anisotropies are several orders of magnitude larger yielding much better reliability of first-principles calculations. 


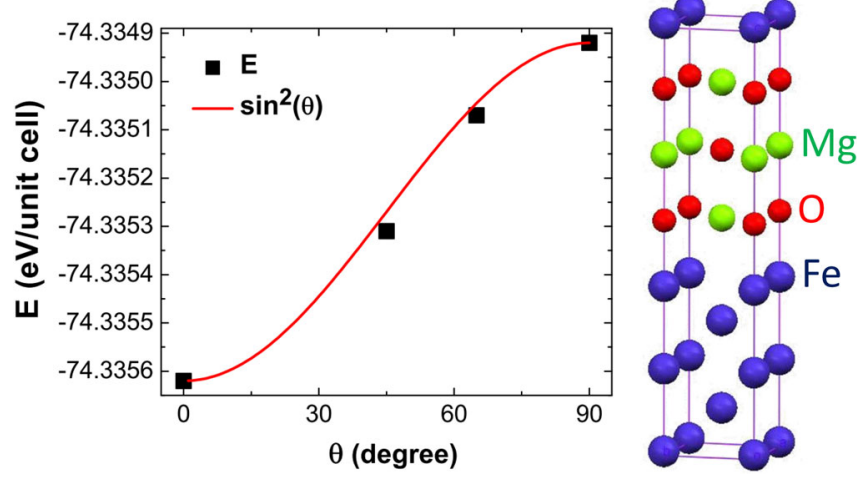

FIG. 16. Angular dependence of the magnetic energy, where $\theta$ is the angle between the magnetization direction and the normal to the interface plane. Unit cell used in the calculation. From Yang et al., 2011.

Figure 16 presents the energy calculated per unit cell as a function of the angle $\theta$ between magnetization orientation and the plane of the pure unrelaxed $\mathrm{Fe} / \mathrm{MgO}$ interface. The vASP package was used with the generalized gradient approximation for exchange correlation and projector-augmented wave potentials; see Yang et al. (2011) for details. In these calculations, the convergence was checked by using larger $K$-point meshes.

The relationship can be well fitted by the conventional uniaxial anisotropy expression given by Eq. (20), with $K_{1}=$ $0.7 \mathrm{meV} /$ atom (1.36 erg $/ \mathrm{cm}^{2}$, i.e., $1.36 \mathrm{~mJ} / \mathrm{m}^{2}$ ) per two interfaces (Yang et al., 2011). It is interesting to note that the higher order anisotropy contribution $K_{2}$ represented in Eq. (21) may be present even though it is much smaller than $K_{1}$ in agreement with recent experimental reports (Zhu et al., 2012; Koziol-Rachwal et al., 2013; Barsukov et al., 2014; Yu et al., 2015; Shaw et al., 2015; Timopheev et al., 2016). With a relaxed structure, anisotropy values grow $\left(\sim 3 \mathrm{erg} / \mathrm{cm}^{2}\right)$ for the two interfaces in agreement with similar PMA values published in earlier theoretical reports using different $a b$ initio approaches (Nakamura et al., 2010; Niranjan et al., 2010). They reported $2.9 \mathrm{erg} / \mathrm{cm}^{2}$ for $\mathrm{MgO} / \mathrm{Fe} / \mathrm{Cu}$ and $1.2 \mathrm{meV} /$ atom $\left(\sim 2.34 \mathrm{erg} / \mathrm{cm}^{2}\right)$ for $\mathrm{Fe} / \mathrm{MgO}$ structures, respectively. It was also found that the PMA intensity for FM/MgO decreases when Fe is replaced by Co (Yang et al., 2011), a result which is consistent with a general trend noted for $\mathrm{Co}_{x} \mathrm{Fe}_{1-x} / \mathrm{MgO}$ interfaces whereby PMA values decrease as the Co concentration increases. This trend has been reported in both experimental and theoretical studies (Yakata et al., 2009; Zhang et al., 2014). This behavior has been attributed to the fact that the orbital magnetic moment of Fe is more anisotropic than that of Co with respect to the magnetization direction as demonstrated in the study by Kanai et al. (2014) combining experimental and theoretical approaches. They reported PMA of $1 \mathrm{~mJ} / \mathrm{m}^{2}$ for $\mathrm{Ta} / \mathrm{CoFeB} / \mathrm{MgO}$ interfaces. It has already been pointed out that the presence of boron at the interface in $\mathrm{CoFeB} / \mathrm{MgO}$ multilayers is detrimental for PMA, suggesting that in the device described by Kanai et al. the B migrates toward the Ta layer. However, Khoo et al. (2013) used first-principles calculations to demonstrate that the interface properties are only mildly affected by the presence of boron during the formation of kotoite $\left(\mathrm{Mg}_{3} \mathrm{~B}_{2} \mathrm{O}_{6}\right)$ compared to $\mathrm{MgO}$.
They also showed that even if kotoite forms at the $\mathrm{CoFeB} / \mathrm{MgO}$ interface, the latter would still yield a similar PMA to that measured at the $\mathrm{CoFe} / \mathrm{MgO}$ interface. Thus they found $1.31 \mathrm{erg} / \mathrm{cm}^{2}$ for $\mathrm{CoFe} / \mathrm{MgO}$ and $0.68 \mathrm{erg} / \mathrm{cm}^{2}$ for $\mathrm{CoFeB} / \mathrm{MgO}$ structures (Khoo et al., 2013). These and other studies suggest that PMA in all these interfaces is produced through similar mechanisms, and that $\mathrm{Fe} / \mathrm{MgO}$ can be used as a model system for $\mathrm{FM} / \mathrm{MOx}$ interfaces involving bcc electrodes, including $\mathrm{Co}_{x} \mathrm{Fe}_{1-x}$ alloys.

To explain the physical mechanisms producing PMA in $\mathrm{Fe} / \mathrm{MgO}$ and similar structures, it is useful to start with the band structure in the presence of spin-orbit coupling when magnetization of Fe is oriented out of plane and in plane (see Fig. 17). From this structure, it emerges that the most important impact of spin-orbit coupling occurs in the vicinity of the Fermi level along the $\bar{\Gamma}$ point of the two-dimensional Brillouin zone. In fact, this corresponds to the direction of the Bloch states with $\Delta$ symmetry which play a decisive role in the Bloch state symmetry-based spin-filtering phenomenon which gives rise to high TMR (Butler et al., 2001, 2005; Butler, 2008). The details on Bloch state symmetries are explained in detail by Butler (2008). The mechanisms behind PMA at $\mathrm{Fe} / \mathrm{MgO}$ interfaces then become comprehensible through the analysis of orbitals for the majority and minority $\Delta$ bands of Fe (Fig. 17) (Yang et al., 2011; Yang, 2012).

Figure 18 shows the bands around the Fermi level $E_{F}$ at the $\bar{\Gamma}$ point with orbital and interfacial atoms projected wave

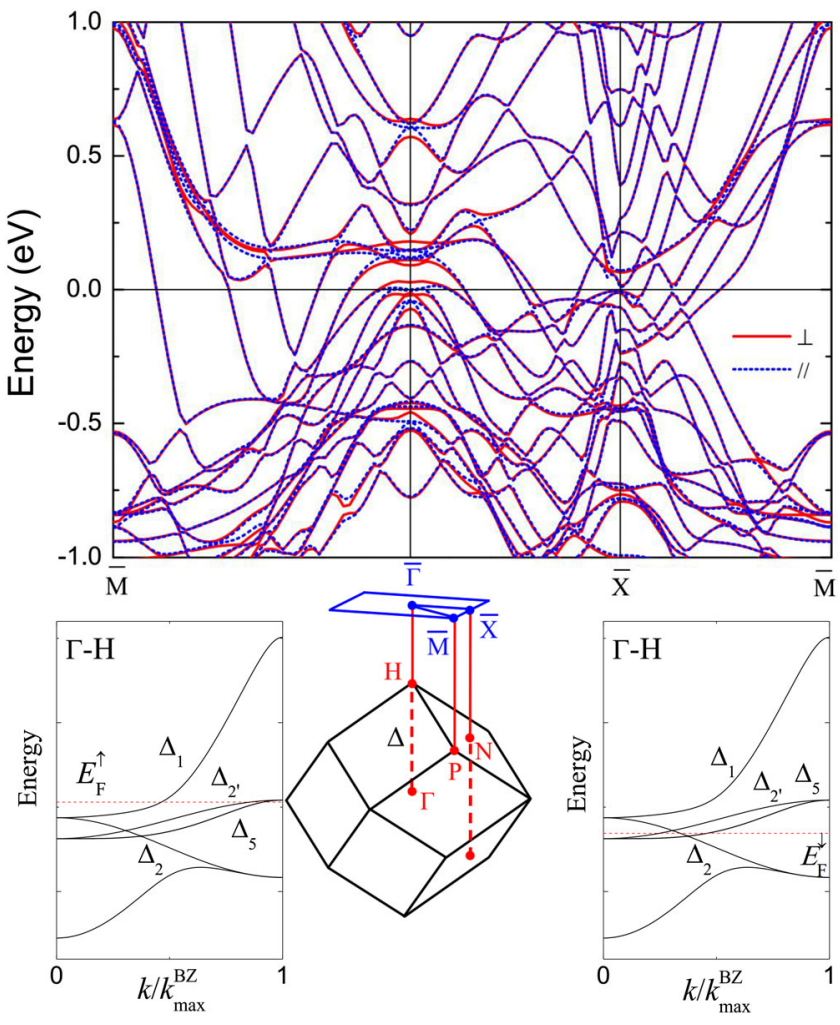

FIG. 17. Band structure for the $\mathrm{Fe} / \mathrm{MgO}$ supercell shown in Fig. 16 in the presence of spin-orbit interaction for out-of-plane and in-plane magnetization orientations. Lower panel: The (100) surface Brillouin zone surrounded by bulk band-structure diagrams for the majority (left) and minority (right) spins in bcc Fe in the absence of spin-orbit interaction. From Yang, 2012. 


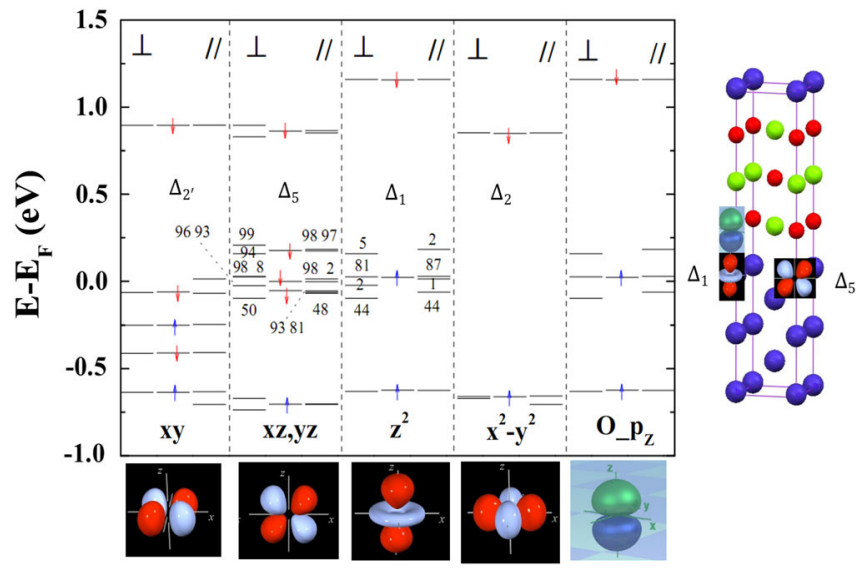

FIG. 18. Effects of spin-orbit coupling on wave function characteristics at the $\bar{\Gamma}$ point for interfacial Fe $d$ and neighboring oxygen $p_{z}$ orbitals for the pure $\mathrm{Fe} / \mathrm{MgO}$ interface illustrated in Fig. 15. Each column shows the band levels for out-of-plane (left) and in-plane (right) orientations of magnetization, as well as band levels for the case where no spin-orbit interaction is included (middle). Numbers on the graph indicate the percentage of orbital character components within the Wigner-Seitz spheres around interfacial atoms. The right panel shows the supercell used with a schematic representation of the Bloch states orbital characteristics. From Yang et al., 2011.

function character for out-of-plane (left subcolumns) and inplane (right subcolumns) orientation of the magnetization as well as in the absence of spin-orbit coupling (middle subcolumns) (Yang et al., 2011).

We concentrate on the band levels in the immediate vicinity of the Fermi level. In the absence of SOI (middle subcolumns), several double-degenerated band levels are apparent with $d_{x z}$ and $d_{y z}$ characteristics representing the minority Bloch states with $\Delta_{5}$ symmetry. At the same time, a band level is present which results from hybridization between the Fe- $d_{z^{2}}$ and $\mathrm{O}-p_{z}$ orbitals. This band level is a signature of the majority Bloch state, with $\Delta_{1}$ symmetry for Fe and $\mathrm{MgO}$ (see Fig. 17) which is at the heart of the spin-filtering phenomenon causing enhanced TMR values in MgO-based MTJs (Butler et al., 2001, 2005; Butler, 2008). When SOI is switched on, the situation changes. First, the degeneracy is lifted for energy levels with $d_{x z}$ and $d_{y z}$ orbital characteristics as indicated above in relation to the impact of the spin-orbit Hamiltonian on Fe bcc bands (Abate and Adsente, 1965). Second, the SOI links the up- and down-spin states, leading to hybridization between Fe- $d_{z^{2}}$ and $d_{x z(y z)}$ which triggers the appearance of a common state containing these and $\mathrm{O}-p_{z}$ orbital characteristics (see Fig. 17). As a result, for the out-of-plane and inplane magnetization orientations (left and right subcolumns), additional common levels originating from the mixing of states with $\mathrm{Fe}-d_{z^{2}}$ and $d_{x z(y z)}$ orbital characteristics due to SOI appear in these subcolumns. These common levels include $\mathrm{O}-p_{z}$ which is part of the up-spin Bloch $\Delta_{1}$ state along with Fe- $d_{z^{2}}$. Thus this mechanism can be seen as a spin-orbitinduced mixing between majority $\Delta_{1}$ and minority $\Delta_{5}$ Bloch states. This mechanism was found to be useful in understanding the mechanisms of tunneling through interfacial resonance states (Lu et al., 2012) as well as in probing band edges by means of low-frequency noise measurements in single-crystal MTJ (Aliev et al., 2014). These hybridized band levels are visibly lower in energy with a larger splitting for the out-of-plane magnetization orientation compared to the inplane orientation as shown in the left and right subcolumns in Fig. 17, respectively. Thus, the increased degeneracy of $d_{x z(y z)}$ orbitals combined with hybridizations between Fe- $d_{z^{2}}, d_{x z(y z)}$, and $\mathrm{O}-p_{z}$ orbitals is at the origin of perpendicular magnetic anisotropy at $\mathrm{Fe} / \mathrm{MgO}$ interfaces. The importance of this hybridization has been underlined experimentally in a number of cases (Okabayashi et al., 2014), including when a Mg layer is inserted between $\mathrm{MgO}$ and $\mathrm{CoFeB}$ electrodes (Ma et al., 2012), in the case of $\mathrm{Co} / \mathrm{MgO}$ interfaces (Chen et al., 2014), and through studying its PMA dependence as a function of Fe-O distance by first principles (Lee et al., 2013).

PMA has been shown to be affected by underoxidation (Yang et al., 2011) and overoxidation (Nakamura et al., 2010; Niranjan et al., 2010; Yang et al., 2011; Tsai et al., 2012) of the interfaces. Oxidation conditions are also known to influence the Bloch states responsible for the high TMR in MTJ based on these types of interface (Faure-Vincent et al., 2003; Zhang, Butler, and Bandyopadhyay, 2003; Butler et al., 2005), which could explain why the maximum PMA and TMR values have been experimentally observed to be attained in a correlated way (Nistor et al., 2010). Supporting the importance of optimal interfacial conditions yielding strong hybridization between Fe-3d with and O- $2 p$ orbitals for both phenomena. As discussed in Sec. II, finding these optimal conditions has indeed been important for progress in optimizing $p$-MTJs for STT-MRAM (Ikeda et al., 2010; Jan et al., 2012). The existence of both PMA and efficient Bloch state spin filtering across $\mathrm{FM} / \mathrm{MgO}$ interface has also been shown to be important for large and robust electrical spin injection into GaAs at zero magnetic field using an ultrathin $\mathrm{CoFeB} / \mathrm{MgO}$ injector (Liang et al., 2014).

A comparative analysis of the impact of different interfacial conditions on PMA allows its physical mechanisms to be further clarified. Using the "anatomy" technique, which allows layer- and orbital-resolved contributions to PMA to be extracted, Hallal et al. (2013) demonstrated that the origin of the large PMA values is far beyond a simple hybridization between the $\mathrm{Fe}-3 d$ and $\mathrm{O}-2 p$ orbitals at the interface between the metal and the insulator. Considering five structures with $\mathrm{Fe}$ interfaced with vacuum or $\mathrm{MgO}$ (Fig. 19, left), they concluded from on-site projected analysis that anisotropy energy is not localized at the interface, but rather that it is spread into the bulk. As a function of the distance from the $\mathrm{MgO} / \mathrm{Fe}$ interface, an attenuating oscillatory behavior is observed which depends on the orbital character of contributing states and interfacial conditions (Fig. 19, bottom right). This analysis clarified the important role of orbital hybridization between both the first and second Fe monolayers after the interface when $\mathrm{MgO}$ is deposited on top of Fe (Hallal et al., 2013). For instance, it becomes clear that while the anisotropy is mainly produced by the surface Fe layer in the case of Fe/ vacuum (due to symmetry breaking) the situation in the presence of $\mathrm{MgO}$ is different in most cases. In these cases, the contribution from the second monolayer is enhanced (Fig. 19). It can also be seen that, compared to the ideal case, the PMA is slightly reduced for underoxidized interfaces 


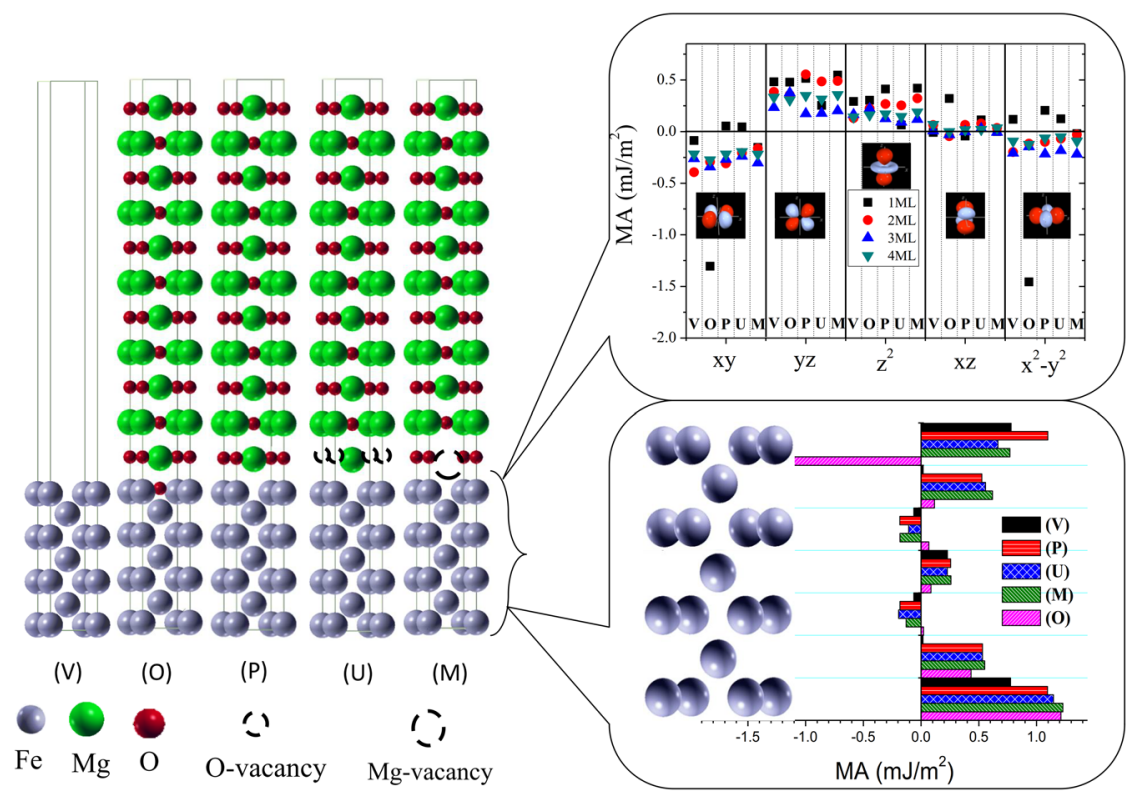

FIG. 19. Layer- and orbital-resolved anisotropy contributions for crystalline structures for $(V)$ Fe/vacuum, $(O)$ overoxidized Fe7/MgO, $(P)$ pure Fe7/MgO11, $(U)$ underoxidized Fe7/MgO, and $(M)$ Mg vacancy in Fe7/MgO11. From Hallal et al., 2013.

and that it is strongly reduced, or even disappears altogether, for overoxidized interfaces. The loss of PMA in overoxidized multilayers is caused by strong IMA in the first Fe layer, due to strong in-plane hybridization between the $\mathrm{Fe}-3 d$ and $\mathrm{O}-2 p$ orbitals in the $\mathrm{FeO}$ layer. Indeed, by examining the orbitalresolved contribution to anisotropy shown in Fig. 19, it becomes obvious that in the case of overoxidation $(O)$ the contribution of in-plane orbitals becomes strongly negative in the first layer (black squares) compared to all other cases [see Hallal et al. (2013) for details]. A similar analysis of orbitalresolved contributions to the anisotropy showed that, in most situations, states hybridized with Fe- $d_{x z(y z)}$ and $d_{z^{2}}$ characteristics tend to maintain their PMA, while those with in-plane characteristics, i.e., $d_{x y}$ and $d_{x^{2}-y^{2}}$, tend to favor in-plane anisotropy (IMA) (Fig. 19, top right). It is also evident that, although in the bulk these contributions mostly compensate each other, at the surface the out-of-plane hybridized orbitals tend to prevail, causing the anisotropy to be out of plane in most cases except in the overoxidized case where the in-plane orbitals strongly contribute to the IMA (Hallal et al., 2013).

Interestingly, the explanation of the origin of PMA correlates well with the orbital moment anisotropy on the grounds of Bruno's theory (Kanai et al., 2014; Liang et al., 2014). As shown in Fig. 20(a), the orbital moment anisotropy $(\Delta \mu)$ completely vanishes in the bulk represented by atom Fe3. This can be explained by the fact that the orbital moment acquired in the plane of the layer exactly compensates the moment acquired along the out-of-plane direction, since in bcc Fe the charge distribution in the $3 d$ shell is almost isotropic. In contrast to bulk $\mathrm{Fe}$, the $\mathrm{Fe} / \mathrm{MgO}$ interface exhibits strong anisotropic behavior due to a significant charge transfer from $\mathrm{Fe}$ to $\mathrm{O}$ orbitals (inset of Fig. 20, left) resulting from hybridization between the out-of-plane Fe $3 d_{z 2}$ and $\mathrm{O} 2 p_{z}$ orbitals. This hybridization leads to a lack of electrons within the Fe out-of-plane orbitals $\left(3 d_{z 2}, 3 d_{x z}\right.$, and $\left.3 d_{y z}\right)$ compared to the in-plane orbitals $\left(3 d_{x^{2}-y^{2}}\right.$ and $\left.3 d_{x y}\right)$. Thus the in-plane orbitals become uncompensated, resulting in an enhanced outof-plane orbital moment which creates considerable PMA in the presence of spin-orbit coupling (Liang et al., 2014). In support of this explanation for the origin of PMA, Fig. 20(b) presents the DOS with the spin-orbit coupling for averaged out-of-plane $\left(d_{z^{2}}+d_{x z}+d_{y z}\right)$ and in-plane Fe $3 d$ orbitals $\left(d_{x^{2}-y^{2}}+d_{x y}\right)$ for Fe both at the interface and in the bulk in the presence of spin-orbit coupling.

Integration of the occupied states below the Fermi level $\left(E_{F}\right)$ gives a difference of about $3 \%$ between the respective out-of-plane and in-plane orbitals for $\mathrm{Fe}$ atoms bound to $\mathrm{O}$ at the interface (Fe5). In contrast, almost no difference is found for $\mathrm{Fe}$ in the bulk $(\mathrm{Fe} 3)$. This results in unbalanced orbital moment anisotropy $\left(\Delta \mu \approx 0.03 \mu_{B}\right)$ associated with the magnetization or spins in the out-of-plane direction for the interfacial atom Fe5 (and Fe1). The anisotropy energy can then be obtained by considering that the spin moment remains unaffected at the interface and applying Bruno's model (Bruno, 1989),

$$
\Delta E_{\mathrm{so}}=\xi \frac{\Delta \mu}{4 \mu_{B}}
$$

where $\xi$ is the spin-orbit-coupling parameter [cf. Eq. (10)]. The interface anisotropy $K_{i}$ can then be qualitatively estimated to be in the range of $1 \mathrm{~mJ} / \mathrm{m}^{2}$ (i.e., $1 \mathrm{erg} / \mathrm{cm}^{2}$ in CGS units). This value is in good agreement with the calculated magnetic anisotropy (MA) energy value [Fig. 20(a)]. When the same approach was applied to overoxidized and underoxidized cases, the degradation and loss of PMA was explained (Liang et al., 2014).

Finally, we discuss the behavior not only of the surface anisotropy but also of the effective anisotropy $K_{\text {eff }}$ and its variation as defined according to Eq. (13). This definition indicates that $K_{\mathrm{eff}} t_{\mathrm{Fe}}$ depends on the thickness of the Fe layer, as reported by Hallal et al. (2013) and illustrated in Fig. 21. 

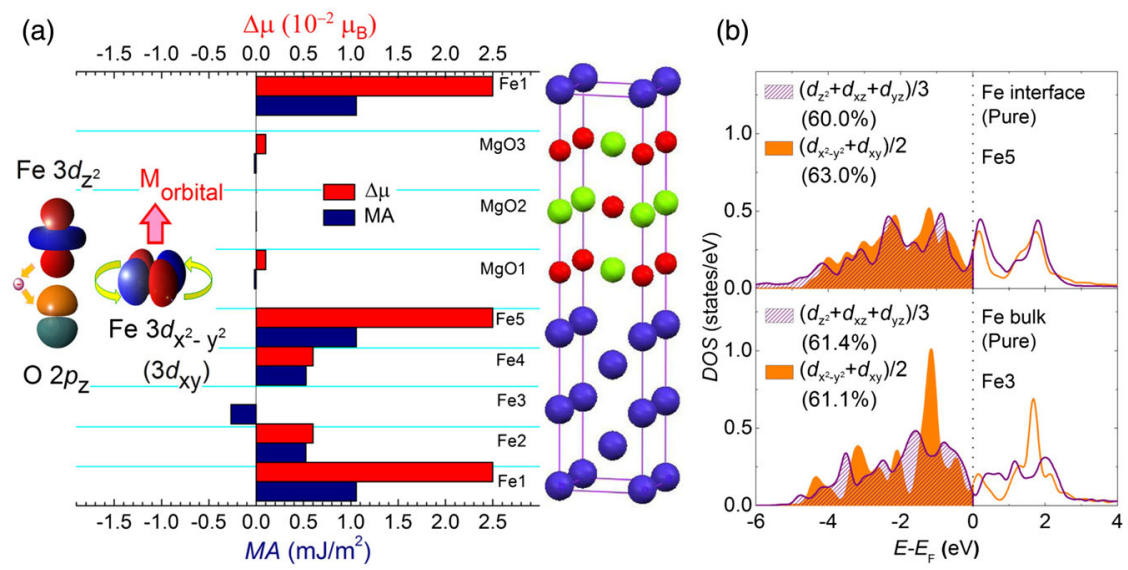

FIG. 20. (a) Layer-resolved orbital moment anisotropy $(\Delta \mu)$ and magnetic anisotropy (MA) energy. Middle: Schematic representation of the crystalline structures calculated. Fe, $\mathrm{Mg}$, and $\mathrm{O}$ are represented by large, medium, and small balls, respectively. (b) DOS with spin-orbit coupling for averaged out-of-plane $\left(3 d_{z^{2}}+3 d_{x z}+3 d_{y z}\right)$ and in-plane Fe-3d orbitals $\left(3 d_{x^{2}-y^{2}}+3 d_{x y}\right)$ for Fe at the interface (Fe5) or in the bulk (Fe3). Inset: a simple picture showing that PMA is produced by the hybridization of Fe out-of-plane orbitals $\left(3 d_{z}^{2}, 3 d_{x z}\right.$, and $\left.3 d_{y z}\right)$ and O $2 p_{z}$ orbitals. This hybridization leads to an uncompensated charge occupation in Fe in-plane orbitals $\left(3 d_{x^{2}-y^{2}}, 3 d_{x y}\right)$ and results in an enhanced out-of-plane orbital moment for PMA. From Liang et al., 2014.

Interestingly, the effective anisotropy was calculated with the demagnetizing term for Eq. (13) determined by applying Eq. (14), i.e., by summing all the magnetostatic dipole-dipole interactions up to infinity. Hallal et al. also showed that if the demagnetization energy is defined as usual [cf. Eq. (7)] by $-\left(\mu_{0} / 2\right) M_{s}^{2}$ for each unit volume in SI $\left(-2 \pi M_{s}^{2}\right.$ for each unit volume in CGS units), its value would be underestimated by about $30 \%$ compared to the value determined from the magnetostatic dipole-dipole interaction (Daalderop, Kelly, and Schuurmans, 1990). Therefore it becomes evident that $K_{\text {eff }} t_{\mathrm{Fe}}$ decreases and reaches the intercept at around $1.2 \mathrm{~nm}$ since the total demagnetizing energy increases in absolute value as the Fe thickness increases [when the demagnetization energy is defined by $-\left(\mu_{0} / 2\right) M_{s}^{2}$, the intercept is around $1.6 \mathrm{~nm}]$. These values are in good agreement with recent experiments indicating a critical thickness of $0.9 \mathrm{~nm}$ for $\mathrm{MgO} / \mathrm{Fe} / \mathrm{MgO}$ (Koziol-Rachwal et al., 2013). Furthermore, Lambert et al. (2013) reported an intercept value of about $0.5 \mathrm{~nm}$ for $\mathrm{Cr}(\mathrm{V}) / \mathrm{Fe} / \mathrm{MgO}$ systems. If we consider that the
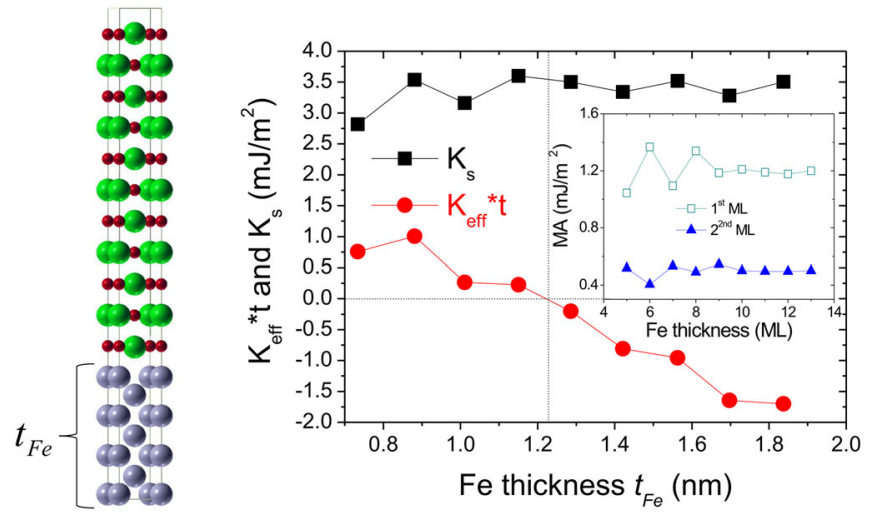

FIG. 21. Dependences of effective and surface anisotropy on Fe thickness for $\mathrm{Fe} / \mathrm{MgO} / \mathrm{Fe} \mathrm{MTJ}$, where $\mathrm{MgO}$ thickness is fixed to $11 \mathrm{ML}$ and Fe varies between 5 and $13 \mathrm{ML}$. (Inset) On-site projected PMA for the first and second interfacial layers plotted against Fe thickness. From Hallal et al., 2013. calculations were performed for two $\mathrm{Fe} / \mathrm{MgO}$ interfaces (MTJ configuration), the corresponding intercept for a single $\mathrm{Fe} / \mathrm{MgO}$ interface is found at $\sim 0.6 \mathrm{~nm}$, which is in agreement with experimental data (see Fig. 22). The described analysis turned out to be efficient for understanding PMA mechanisms in $\mathrm{FM} / \mathrm{MgO}$ MTJs in the presence of impurities (respectively, capping layers) within (respectively, next to) the FM layer (Hallal, Dieny, and Chshiev, 2014; Peng et al., 2015) as well as for the Heusler alloy based MTJs (Vadapoo et al., 2016).

In conclusion, this section presented an overview of the theoretical basis for PMA. The qualitative picture of spin-orbit induced hybridization between atomic orbitals was given first, followed by an historical perspective on theoretical descriptions of magnetocrystalline anisotropy in ultrathin films and

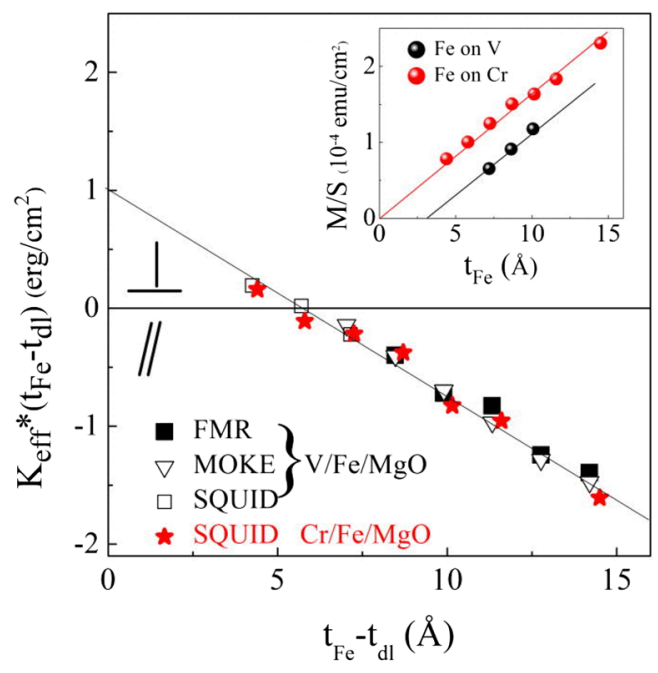

FIG. 22. Effective anisotropy constant $K_{\mathrm{eff}}\left(t_{\mathrm{Fe}}-t_{d l}\right)$ plotted as a function of $t_{\mathrm{Fe}}-t_{d l}$ at $\mathrm{RT}$ for $\mathrm{V} / \mathrm{Fe} / \mathrm{MgO}$ and $\mathrm{Cr} / \mathrm{Fe} / \mathrm{MgO}$ systems. The inset shows real values for magnetization vs $\mathrm{Fe}$ thickness, showing the dead layer thickness at the intercept with the $x$ axis, $t_{d l}=0$ for $\mathrm{Fe}$ on $\mathrm{Cr}$, and $t_{d l}=3 \AA$ for $\mathrm{Fe}$ on V. From Lambert et al., 2013. 
monolayers. After that, recent developments and how they impact our understanding of the physical mechanisms controlling interfacial anisotropy at $\mathrm{FM} / \mathrm{MgO}$ interfaces, including PMA, were then presented, mainly emphasizing calculations based on first principles.

\section{STT-MRAM BASED ON INTERFACIAL CoFeB/MgO PMA}

\section{A. STT-MRAM: A subclass of MRAM}

Following the first observations of large TMR effects at RT in alumina-based MTJs (Miyazaki and Tezuka, 1995; Moodera et al., 1995), intense research activity was launched worldwide to use this effect in magnetic solid-state memories or MRAM. In MRAMs, the storage elements are magnetic tunnel junctions and the information is coded based on the relative orientation of the magnetization in each MTJ cell's two magnetic electrodes (e.g., parallel $=$ "0", antiparallel $=$ " 1 "). The information can be read by measuring the cell's resistance, which, due to the TMR effect, depends on its magnetic configuration. In 1995, breakthrough discoveries were made in spintronics, and since then tremendous progress has been made in the development of these memories. Figure 23 summarizes the main families of MRAM which were developed over the past 20 years.

The first generation of MRAM (Stoner-Wolfarth and toggle MRAMs) was based on field-induced magnetization switching (Savtchenko et al., 2003). In these devices, the MTJs were magnetized in plane and patterned in an elliptical shape with a typical aspect ratio (AR) of between 2 and 3. This ellipticity yields a uniaxial anisotropy with easy axis along the long axis of the ellipse. In toggle MRAM, the storage layer is a synthetic antiferromagnetic layer which is switched upon writing by applying a sequence of four steps corresponding to a gradual rotation of the applied field by steps of $45^{\circ}$. Toggle MRAM have been commercialized since 2006 by Everspin and are now available in 1,4 , and 16 Mbit sizes. They are being used in a variety of microcontrollers and, thanks to their resistance to radiation, they are particularly attractive for space and avionic applications. However, the relatively large currents required to generate the pulses of write magnetic fields $(\sim 10 \mathrm{~mA})$ mean they cannot be downscaled below $\sim 90 \mathrm{~nm}$ technology node.

Thermal assistance was also proposed as a solution to bypass the dilemma between memory retention and writability in MRAM (Dieny and Redon, 2001; Prejbeanu et al., 2007), and TAS-MRAM technology is currently developed by Crocus Technology. With TAS, during writing, a pulse of current is sent through the MTJ which temporarily heats the storage layer through Joule dissipation around the tunnel barrier. This heating reduces the energy barrier to allow magnetization switching, thus permitting the use of lower magnetic fields than with toggle MRAM. Furthermore, the pulse of magnetic field can be shared between all the bits in a given word since write selectivity is achieved in TAS systems by a combination of heating (produced by a vertical heating current passing through each selected cell) and the field produced by a horizontal current flowing along a word line. This possibility significantly reduces the power consumed by field-written MRAM. However, scalability remains limited [although it is better than toggle MRAM thanks to lower write fields and multilevel capability (Stainer et al., 2014)] due to the electromigration limit associated with field generation in the word lines.

Following the first observations of STT switching in metallic $\mathrm{Co} / \mathrm{Cu} / \mathrm{Co}$ nanopillars (Katine et al., 2000), a different kind of MRAM was proposed in which the storage layer's magnetization can be switched by STT rather than by applying a field (middle column in Fig. 23). Thanks to progress made in the growth of MTJ, particularly with regard to reducing the thickness of the oxide barrier while maintaining a large TMR amplitude, STT switching was obtained in MTJs (Fuchs et al., 2004; Huai et al., 2004). Based on these results, STT appeared to be an interesting new write scheme
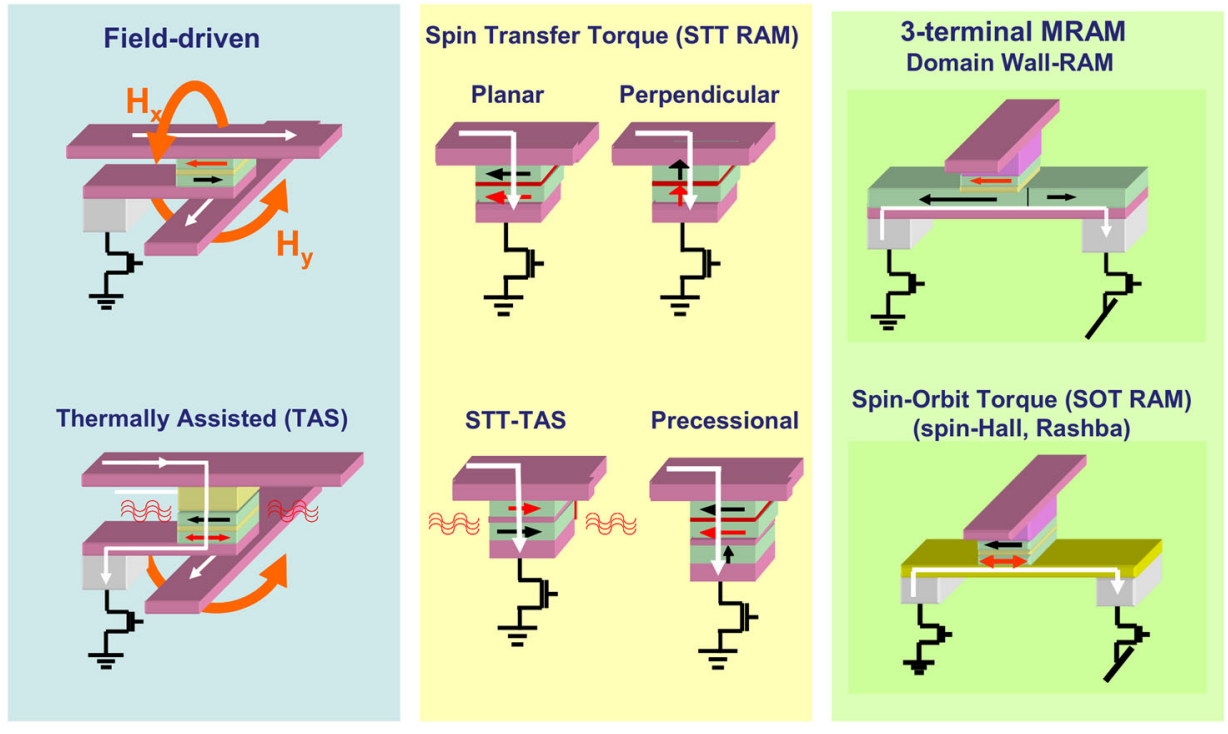

FIG. 23. Various families of MRAM developed since 1995. Left: First MRAM generation based on field writing. Center: Various flavors of STT-MRAM with in-plane and out-of-plane magnetization, with thermal assistance (TAS) or orthogonal polarizers (precessional or OST-MRAM). Right: 3-terminal MRAM using current-induced domain wall propagation or spin-orbit-torque (SOT) effects (Rashba or spin Hall effects). 
for MRAM since it would allow a much better downscalability than field-written MRAM. Indeed, with STT, the condition for magnetization switching in the case of an in-plane magnetized MTJ is expressed by a critical current density given by

$$
J_{c 0}=\frac{2 e \alpha \mu_{0} M_{s} t_{F}\left(H+H_{k}+M_{s} / 2\right)}{\hbar \eta},
$$

where $e$ is the electron charge, $\alpha$ is the Gilbert damping, $M_{s}$, $t_{F}$, and $H_{k}$ are the storage layer magnetization, thickness, and anisotropy field, respectively, $\eta$ is the STT efficiency related to spin polarization of the tunneling current, and $H$ is the applied field. This current density $J_{c 0}$ corresponds to the current density required to switch magnetization in about $5 \mathrm{~ns}$. The current density required to switch the storage layer magnetization actually varies with the current pulse duration, decreasing as the pulse duration increases (Koch, Katine, and Sun, 2004; Li and Zhang, 2004; Tomita et al., 2013). The total current that must be applied to write $I_{\mathrm{wr}}$ can be determined from $I_{\mathrm{wr}}=J_{c 0} \times A$, where $A$ is the area of the MTJ. Based on this relation, the smaller the area, the smaller the critical current that must be applied to induce switching. In fact, a switching current of around $20 \mu \mathrm{A}$ was determined for sub20-nm MTJ with perpendicular anisotropy, demonstrating the nice downscalability of STT-MRAM (Sato et al., 2014). However, as we will see [Eq. (27) and related discussion], in out-of-plane magnetized MTJ (p-MTJ), this trend is actually limited by the minimum thermal stability factor required to retain the memory for the specified retention time. The total current that must be applied to write in p-MTJ will be proportional to the thermal stability factor which sets a lower limit to the write current.

Historically, the first STT-MRAM developments were performed using in-plane magnetized MTJs patterned as elliptical cells. The downscalability of these systems was limited by the maximum anisotropy, which was provided by the shape anisotropy due to the elliptical shape of the cells. Therefore, for sizes below $60 \times 150 \mathrm{~nm}^{2}$, the anisotropy was too small to allow sufficiently long retention. From around 2007, most research efforts switched to out-of-plane magnetized MTJ providing much larger PMA, in particular, thanks to the very large interfacial anisotropy at $\mathrm{CoFeB} / \mathrm{MgO}$ interfaces. Another advantage of these out-of-plane magnetized MTJs is that, for a given thermal stability factor, they require less current to induce switching than their in-plane counterparts. This was another strong motivation for focusing efforts on these p-MTJs [for reviews on STT-MRAM, see Khvalkovskiy et al. (2013) or Sbiaa, Meng, and Piramanayagam (2011)].

In STT-MRAM, different embodiments were introduced for various purposes. Thus thermally assisted STT-MRAM were developed in an attempt to reduce the write current while maintaining a large thermal stability factor (Bandiera et al., 2011). STT-MRAM with orthogonal polarizers are being developed for ultrafast MRAM applications such as static random access memory (SRAM) like applications (Redon, Dieny, and Rodmacq, 2000; Kent, Ozyilmaz, and del Barco, 2004; Lee, Redon, and Dieny, 2005; Lee et al., 2009; Papusoi et al., 2009; Bedau et al., 2010).

More recently, 3-terminal MRAMs emerged (illustrated in the right column in Fig. 23). The storage elements in these
MRAMs are still MTJs, but each cell is contacted to three terminals. Writing is performed by applying a current through a horizontal strap placed below the MTJ. Several mechanisms were proposed to allow magnetization switching thanks to this horizontal current: either current-induced domain wall motion (Cros et al., 2014) or spin-orbit torques due to Rashba or spin Hall effects (Miron et al., 2010; Liu, Pai et al., 2012). The advantage of these 3-terminal devices is that they separate the write and read current paths. This separation is interesting from a design point of view while also eliminating the electrical stress imposed on the tunnel barrier by the conventional STT write approach. The drawback of these devices is their larger cell footprint since two transistors are generally connected to each cell. For these reasons, 3-terminal memories are of most interest for ultrafast SRAM-type memory applications for which sub-ns switching is required.

As a follow-up to this overview of the various families of MRAM, we now focus on STT-MRAM with in-plane magnetization but a reduced demagnetizing field thanks to the iPMA at $\mathrm{CoFeB} / \mathrm{MgO}$ interfaces and to p-MTJ based STT-MRAM.

\section{B. In-plane magnetized STT-MRAM with reduced demagnetizing field}

The critical current for switching can be determined from a macrospin model at zero temperature, as indicated in Eq. (22). This relationship was determined by analyzing the stability of the solution to the Landau-Lifshitz-Gilbert (LLG) equation under the influence of STT. Magnetization will be reversed when the injected current has the appropriate direction (electrons flowing from the reference layer to the storage layer to induce a switch from the antiparallel magnetic configuration $(A P)$ to the parallel one $(P)$ and flowing in the opposite direction to switch from $P$ to $A P$ ) and is larger than $J_{c}(\tau), \tau$ being the pulse duration (Koch, Katine, and Sun, 2004; Li and Zhang, 2004). In Eq. (22), the term $M_{s} / 2$ is usually larger than $H+H_{k}$ by 1 or 2 orders of magnitude. This dominant role of the demagnetizing field term $\left(\mu_{0} M_{s} / 2\right.$ in absolute value $)$ is due to the fact that, during the STT-induced switching of the magnetization of the in-plane magnetized layer, the magnetization has to precess out of plane, thus increasing the demagnetizing energy. As a result, a good approximation of $J_{c 0}$ is generally given by

$$
J_{c 0} \cong \frac{e \alpha \mu_{0} M_{s}^{2} t_{F}}{\hbar \eta} .
$$

Because of the dominance of the demagnetizing field term in Eq. (22), it is also important to note that the critical current for STT writing weakly depends on $H_{k}$, the in-plane anisotropy field, which determines the thermal stability of the magnetization at rest. In other words, in in-plane magnetized MTJs, the barrier for STT switching is mainly related to the demagnetizing energy, whereas the barrier for thermal stability of the magnetization, i.e., memory retention, is determined by the in-plane shape anisotropy [see Fig. 24(a)]. The switching barrier is generally much larger than the stability barrier which means that the in-plane magnetized configuration is quite unfavorable in terms of STT switching efficiency. As will be explained in the next 
(a) In-plane magnetized MTJ

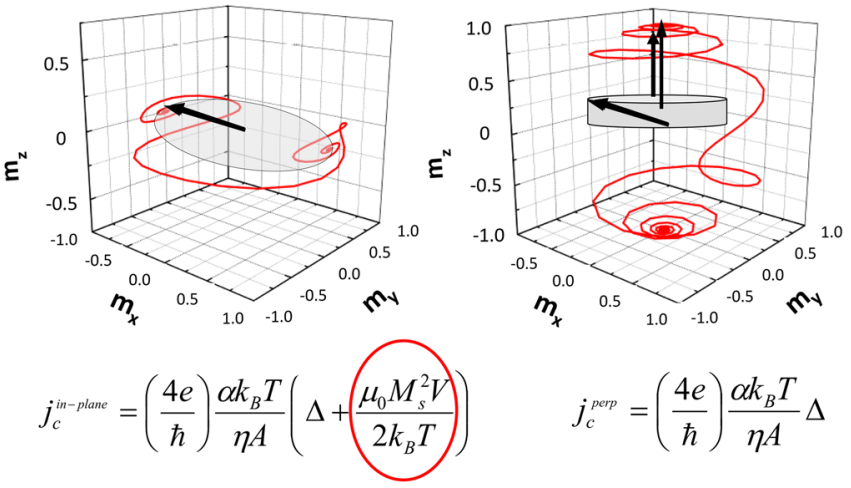

FIG. 24. Qualitative comparison of STT-induced magnetization switching in (a) an in-plane magnetized storage layer and (b) an out-of-plane magnetized storage layer. In both cases, memory retention is related to the thermal stability factor $\Delta$. In (b), the critical current for writability is directly proportional to $\Delta$ Thus, in this case, the barrier determining the thermal stability of the storage layer's magnetization and that which must be overcome to allow STT-induced switching are the same. In contrast, in (a), the barrier for switching due to thermal fluctuations is given by the in-plane shape anisotropy, whereas the barrier for STT-induced switching is mostly determined by the demagnetizing energy [terms circled in red in (a)].

section, out-of-plane magnetized MTJs do not have this drawback.

Nevertheless, in 2005 and 2006 several authors indicated that the ability of the storage layer magnetization to precess out of plane during switching could be significantly eased by introducing some out-of-plane anisotropy into the storage layer (Nguyen and Huai, 2004; Rodmacq and Dieny, 2006; Sun, 2006). Introducing partial PMA into the storage layer means that its magnetization still lies in plane when at rest, but that it can more easily go out of plane during switching. In this approach, the terms $H_{k}$ and $M_{s} / 2$ in Eq. (22) will have opposite signs so that the critical current required for STTinduced writing will be reduced. This effect is currently used by Everspin in their 64-Mbit STT-MRAM product which was commercialy launched at the end of 2012 (Rizzo et al., 2013). The free layer used for the 64-Mbit ST-MRAM is a low CoFeB-based alloy with net in-plane magnetization, but where the easy-plane demagnetizing energy is counterbalanced in a proportion of more than $50 \%$ by the $\mathrm{CoFeB} / \mathrm{MgO}$ interfacial PMA. Figure 25 shows some of the characterization results obtained for this 64-Mbit product which takes advantage of the iPMA at the $\mathrm{CoFeB} / \mathrm{MgO}$ interface.

Figure 25(b) shows that writing can be successful over a large range of write voltages. In Fig. 25(c), the energy barrier determining memory retention was varied by changing the cell's aspect ratio. Thus, for $\mathrm{AR}<3$, the magnetization of the storage layer switches coherently and the thermal stability factor increases with $\mathrm{AR}$. In contrast, for $\mathrm{AR}>6$, the storage layer's magnetization switches by nucleation or propagation of a reverse domain and no benefits in terms of thermal stability factor or memory retention are obtained by increasing the aspect ratio above 3 . On the contrary, an additional penalty emerges in terms of switching current due to the associated increase in cell area.

These developments demonstrated that, even for STTMRAM with in-plane magnetized MTJ, a significant benefit
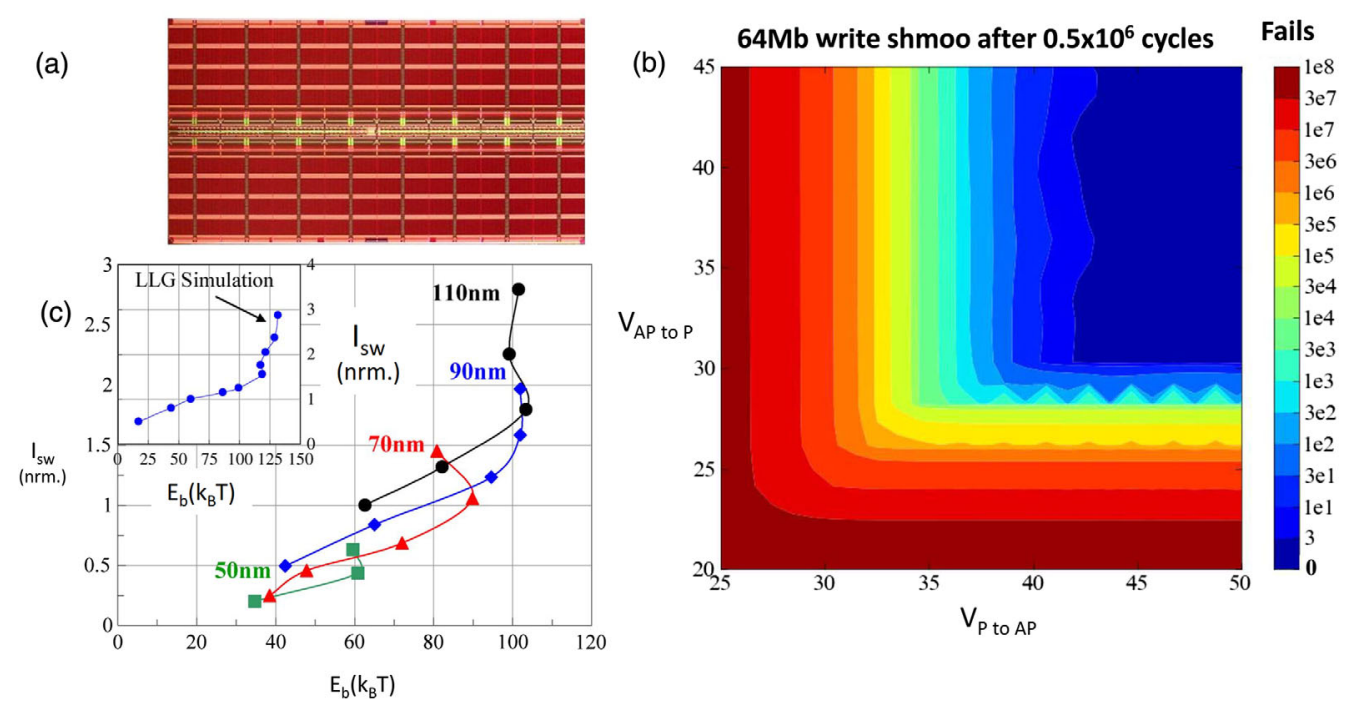

FIG. 25. (a) Everspin's 64-Mbit DDR3 STT-MRAM die photo. Wordline drivers run vertically through the center of each of eight 8 Mbit banks. Strips of column circuits run horizontally, dividing each bank into eight subarrays. (b) Shmoo plot of the voltage applied to induce $A P$-to- $P$ and $P$-to- $A P$ switching in a $64-\mathrm{Mbit}$ device. The axes are labeled with voltage steps of $50 \mathrm{mV}$. The color scale represents the number of bits failing a write low or write high pattern out of the full 64 Mbit. A large region for which 0 fails were recorded was obtained even after all the bits had been written $0.5 \times 10^{6}$ times. (c) STT switching current as a function of the energy barrier measured for ellipsoidal CoFeB-based free layers with widths of 50, 70, 90, and $110 \mathrm{~nm}$ and aspect ratios varying between 2 and 3.5. For each width, the $\mathrm{AR}$ was $\sim 2$ at the minimum $E_{b}$ and increased monotonically in steps of $\Delta \mathrm{AR}=0.3$. The constant $E_{b}$ for large $\mathrm{AR}$ indicates a transition from coherent rotation to domain nucleation. Inset: Switching current $I_{s w}$ vs $E_{b}$ for an $80 \mathrm{~nm}$ wide bit for which AR varied between 1.5 and 3.5, as calculated using a micromagnetic simulation applying the Slonczewski torque term. From (Rizzo et al., 2013. 
in terms of switching current could be obtained by taking advantage of the interfacial PMA at the $\mathrm{CoFeB} / \mathrm{MgO}$ interface. However, the main benefit of this iPMA will be realized in STT-MRAM cells with fully p-MTJ, as we see in the following section.

\section{Perpendicular STT-MRAM}

The critical current for STT-induced reversal of magnetization in the storage layer based on the LLG equation in the out-of-plane configuration is given by (Sun, 2000)

$$
I_{c 0}=\frac{2 e}{\hbar} \frac{\alpha A t \mu_{0} M_{s}}{\eta} H_{\mathrm{eff}}
$$

where $A$ is the area of the magnetic element, $e$ is the electron charge, $\hbar$ is the reduced Planck constant, $\mu_{0}$ is the vacuum permeability, $\alpha$ is the Gilbert damping coefficient, $M_{s}$ and $t$ are the saturation magnetization and thickness of the storage layer, $\eta$ is the STT efficiency that depends on the relative orientation of the magnetizations $(\theta=0$ or $\pi)$ and on the polarization $P$, and $H_{\text {eff }}$ is the effective switching field [see Fig. 24(b)].

In magnetic junctions with out-of-plane magnetization, the effective field acting on the storage layer magnetization is given by

$$
H_{\mathrm{eff}}=H_{K \perp}-M_{s} \text {, }
$$

where $H_{K \perp}$ is the perpendicular anisotropy field which pulls the magnetization out of plane $\left(H_{K \perp}>M_{s}\right)$. In contrast to the in-plane magnetized case, the STT and thermal energy barriers are identical in this case. Therefore, for a given memory retention, the critical switching current can be much smaller than with in-plane magnetized electrodes. In addition, since shape anisotropy no longer plays a role in the thermal stability of the system, magnetic cells can be made circular rather than elliptical. This change in shape facilitates downscaling. Several papers have addressed the potential advantages of perpendicular MTJ (Heinonen and Dimitrov, 2010; Wolf et al., 2010; Yoda et al., 2010). By introducing the thermal stability factor

$$
\Delta=\frac{K V}{k_{B} T}=\frac{\mu_{0} M_{s} H_{\mathrm{eff}} A t}{2 k_{B} T},
$$

where $K$ is the anisotropy, $V=A t$ is the volume of the storage layer, $k_{B}$ is Boltzmann's constant, and $T$ is the absolute temperature, Eq. (24) can be rewritten as follows:

$$
I_{c 0}=\frac{4 e}{\hbar} \frac{\alpha k_{B} T}{\eta} \Delta
$$

This relation reflects the fact that in the macrospin approximation (valid at dimensions below around $40 \mathrm{~nm}$, as discussed later), the thermal stability factor (i.e., the memory retention capacity) and the critical current for data writing are directly proportional. This proportionality illustrates the classical dilemma between retention and writability often encountered in memory technology: the more stable the information, the more difficult it is to change it, i.e., to write in the memory. To account for Eq. (27), a figure of merit was proposed, which is the ratio of the thermal stability factor to the critical switching current $\Delta / I_{C 0}$ (Kishi et al., 2008). As a follow-up to the discussion in the previous paragraph and here, the figure of merit for out-of-plane magnetized MTJ is expected to be much better (larger $\Delta / I_{C 0}$ ) than for in-plane magnetized systems. However, this will hold true only if the Gilbert damping constant $\alpha$, to which $I_{c 0}$ is proportional, can be maintained at a similar low value to that in in-plane magnetized material. This is not easy to achieve as will be explained in the next section.

\section{Benefit of exploiting the interfacial PMA at CoFeB/MgO interfaces when tackling the large anisotropy or low critical current dilemma}

In STT-MRAM, a large anisotropy is required to allow sufficiently long memory retention. Indeed, as explained previously when discussing Eq. (6), information retention is directly related to the thermal stability factor $\Delta$, which can generally be expressed in line with Eq. (8) as

$$
\Delta=\frac{K_{\mathrm{eff}} V}{k_{B} T}=\frac{\left[K_{s}+K_{v} t-\mu_{0}\left(M_{s}^{2} / 2\right) t\right] A}{k_{B} T},
$$

where $A$ is the cell area and all other quantities are the same as those used in Eq. (8). This equation is valid on the condition that the lateral size of the cell is much larger than the thickness of the storage layer. Otherwise, the demagnetizing coefficients corresponding precisely to the sample geometry should be taken into account in the expression of the demagnetizing energy. In other words, the term $-\mu_{0}\left(M_{s}^{2} / 2\right)$ in Eq. (28) should be replaced by $-\mu_{0}\left(M_{s}^{2} / 2\right)\left(N_{z}-N_{x}\right)$ (Beleggia et al., 2005). Equation (28) also assumes that magnetization switching due to thermal fluctuations is induced via coherent rotation and not nucleation or propagation reversal. Sato et al. (2011) demonstrated that in $\mathrm{Ta} / \mathrm{CoFeB} / \mathrm{MgO}$-based $\mathrm{p}$-MTJ this assumption is valid for MTJs with a diameter below $\sim 40 \mathrm{~nm}$ (see Fig. 26). Indeed, Sato et al. observed that with MTJ diameters above $\sim 44 \mathrm{~nm}$ the thermal stability factor increased much more slowly versus MTJ area than for smaller diameters, indicating that the magnetization switching under the influence of thermal fluctuations was produced by nucleation of a reverse domain and propagation of a domain wall throughout the storage layer rather than by coherent rotation. In contrast, the critical current for switching was found to increase in direct proportion to the cell area over the whole range of diameters investigated.

In p-MTJ, since the thickness of the storage layer is always much smaller (typically $2 \mathrm{~nm}$ ) than the lateral dimensions of the cell (generally greater than $20 \mathrm{~nm}$ ), the demagnetizing energy is negative. This negativity will favor an in-plane orientation for the storage layer's magnetization. Therefore a strong out-of-plane anisotropy of bulk or interfacial origin will be required to pull the magnetization of the storage layer out of plane. In addition, since the energy barrier for magnetization switching induced by thermal fluctuations is proportional to the cell area (at least for diameters smaller than $\sim 40 \mathrm{~nm}$ ), this perpendicular anisotropy per unit area must increase as the cell size decreases. From a physical point of view, as 

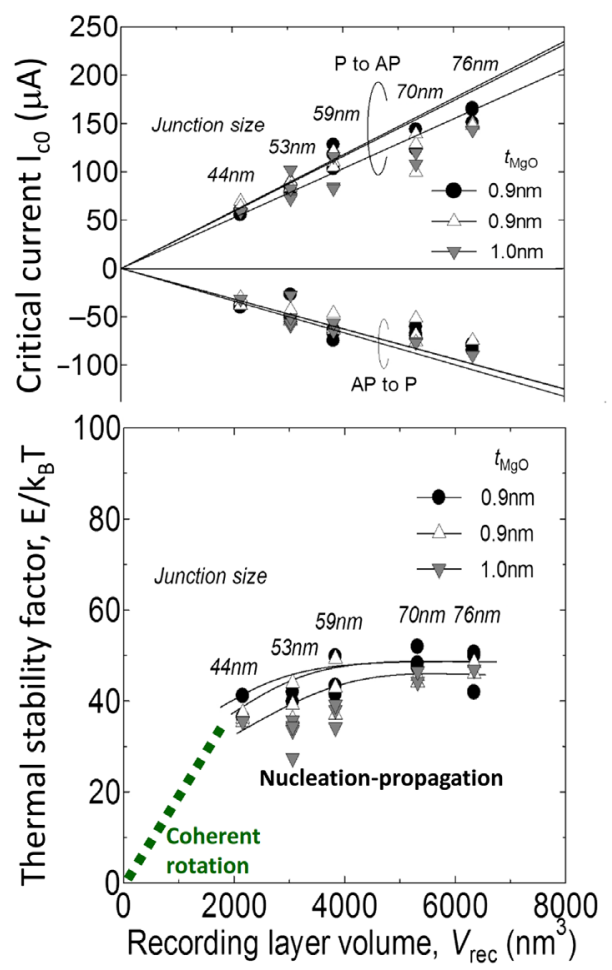

FIG. 26. Critical current for magnetization switching by STT and thermal stability factor plotted as a function of storage layer volume in Ta/CoFe/B-based p-MTJ. From Sato et al., 2011.

previously explained (e.g., see Sec. III), magnetic anisotropy, which corresponds to a coupling between the electron spins (the magnetization) and the crystallographic lattice, is produced by spin-orbit interaction. Usually, materials with large spin-orbit coupling exhibit large anisotropy [such as $\mathrm{NdFeB}$ permanent magnets, Bi-based compounds, or $(\mathrm{Co} / \mathrm{Pt})$ or $(\mathrm{Co} / \mathrm{Pd})$ multilayers]. The problem with using such large spin-orbit materials in the context of STT-MRAM is that they also tend to exhibit large Gilbert damping, which is directly proportional to the critical current for switching by STT, as shown by Eq. (27). Therefore large Gilbert damping means a large write current is necessary, which affects the memory's power consumption and, indirectly, the cell size since the selection transistor connected in series with the MTJ will need to be larger than the MTJ so as to deliver the required current.

Fortunately, anisotropy and damping are not directly linked, as illustrated, in particular, in Fig. 27 [see Bai et al. (2012) and references therein]. Furthermore, the Gilbert damping can also be influenced by spin-pumping phenomena in magnetic thin films surrounded by large spin-orbit materials [see Fig. 12(e) and related discussion] (Kato et al., 2012; Shaw, Nembach, and Silva, 2012). Some materials do exhibit large anisotropy together with weak Gilbert damping, for example, $(\mathrm{Co} / \mathrm{Ni})$ multilayers and, even more remarkably, $\mathrm{Co}_{2} \mathrm{FeAl} / \mathrm{MgO}$ or $\mathrm{GaMn}_{3}$. However, these materials are not yet ready to be implemented in STT-MRAM or their TMR is not large enough at room temperature when incorporated into $\mathrm{MgO}$ based MTJs (Bai et al., 2012). Fortunately, $\mathrm{CoFeB} / \mathrm{MgO}$ interfaces are another example combining large anisotropy with weak Gilbert damping. As previously explained, this combination is made possible in this system thanks to a weak

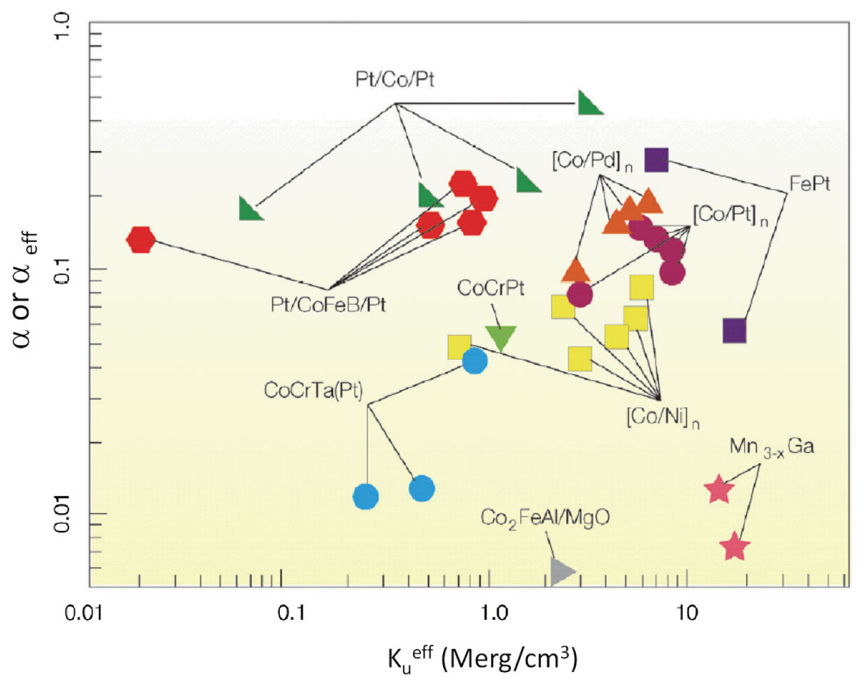

FIG. 27. Gilbert damping vs uniaxial magnetic anisotropy in a variety of PMA films. From Bai et al., 2012.

spin-orbit combined with a large decrease in degeneracy of the $\mathrm{Co}(\mathrm{Fe}) 3 d$ orbitals due to the strong hybridization between the magnetic transition metal $d z^{2}$ orbitals and the $\mathrm{O} s p$ orbitals in $\mathrm{MgO}$. Since $\mathrm{CoFeB} / \mathrm{MgO}$ can also be used to produce large TMR in p-MTJ, CoFeB/MgO-based p-MTJ are particularly attractive for STT-MRAM applications.

\section{E. Influence of various parameters on the PMA measured at $\mathrm{CoFeB} / \mathrm{MgO}$ interfaces}

\section{Influence of buffer and capping layers}

In $\mathrm{CoFeB} / \mathrm{MgO}$-based MTJs, the buffer and capping layers play several roles. During annealing of the MTJ stack and crystallization of the initially amorphous $\mathrm{CoFeB}$ layers in contact with the $\mathrm{MgO}$ tunnel barrier layer, the buffer and capping layers must attract the $\mathrm{B}$ out of the $\mathrm{CoFeB}$ layers to allow crystallization. It is particularly important that the $\mathrm{B}$ be drawn away from the $\mathrm{MgO}$ interface since its presence here is detrimental for both the interfacial anisotropy and the TMR. Furthermore, the buffer layer must have a low roughness (typically below $0.2 \mathrm{~nm} \mathrm{rms}$ ). In these conditions, the $\sim 1-\mathrm{nm}-$ thick tunnel barrier will be as uniform as possible and hot spots of tunneling current can be avoided. The buffer and capping layers must also have a low tendency to diffuse into the $\mathrm{CoFeB}$ layer upon annealing since this diffusion can affect the magnetic properties of $\mathrm{CoFeB}$. In particular, if the diffusing species reach the $\mathrm{MgO}$ interface, magnetic dead layers may form, Gilbert damping could increase, or the interfacial PMA or TMR amplitude could be decreased.

Various buffer and capping layers have been investigated. Pt was used in early studies of interfacial PMA at magnetic metal/oxide interfaces (Monso et al., 2002; Rodmacq et al., 2003, 2009; Manchon et al., 2008; Nistor et al., 2010). It was found to be a good buffer for $\mathrm{Co}(\mathrm{Fe}) /$ alumina-based MTJs but not for $\mathrm{CoFeB} / \mathrm{MgO}$ MTJs [Fig. 11(b)] due to its poor ability to draw $\mathrm{B}$ out of the $\mathrm{CoFeB}$ layer away from the $\mathrm{MgO}$ interface. Worledge et al. (2014) performed a number of studies between 2009 and 2010 to investigate various buffers (Cr, Ta, Ru, V, Ti, Al, Mg, Ru, W). Their results identified Ta 
as the preferred one. However, Ta has several drawbacks: it forms a $\sim 0.3$-nm-thick dead layer in contact with $\mathrm{CoFeB}$ and causes a significant increase in Gilbert damping in $\mathrm{Ta} / \mathrm{CoFeB} / \mathrm{MgO}$ as the $\mathrm{CoFeB}$ thickness decreases [see Fig. 12(e)]; it also diffuses into the $\mathrm{CoFeB}$ toward the $\mathrm{MgO}$ barrier when annealing temperatures exceed $\sim 300^{\circ} \mathrm{C}$, thus limiting the TMR amplitude (Ikeda et al., 2008).

More recently, other buffer layers were tried. Liu, Cai, and Sun (2012) substituted a Hf buffer for the Ta buffer and observed a $35 \%$ increase in PMA at the $\mathrm{CoFeB} / \mathrm{MgO}$ interface. In addition, sputter-deposited Hf films were found to be amorphous at small thicknesses and their roughness was naturally low, thus providing a smooth surface for the subsequent growth of the $\mathrm{CoFeB}$ electrode and $\mathrm{MgO}$ barrier. The same group also tested Mo as a buffer (Liu et al., 2014) and found $\mathrm{Mo} / \mathrm{CoFeB} / \mathrm{MgO}$ to have better thermal endurance than with the Ta buffer, with negligible detrimental intermixing after $2 \mathrm{~h}$ annealing at temperatures up to $425^{\circ} \mathrm{C}$. In addition, interfacial PMA was still quite large, close to $2 \mathrm{~mJ} / \mathrm{m}^{2}$. However, their study did not provide data on the TMR amplitude in $\mathrm{Mo} / \mathrm{CoFeB} / \mathrm{MgO}$ MTJ based on a Mo underlayer.

Sinha et al. (2013) also observed that introducing nitrogen into the Ta buffer layer further enhanced the PMA in $\mathrm{Ta} / \mathrm{CoFeB} / \mathrm{MgO}$-based p-MTJ. They observed a close relationship between the interfacial anisotropy and the amount of nitrogen introduced into the Ta buffer. Thus, the PMA could reach $1.8 \mathrm{~mJ} / \mathrm{m}^{2}$ in optimal $\mathrm{N}$-doping conditions. The magnetic dead layer thickness at the buffer/CoFeB interface was found to decrease monotonically as the nitrogen concentration increased, but it did not reach zero at the optimum concentration yielding the largest PMA. The formation of a thin TaN layer at the $\mathrm{Ta} / \mathrm{CoFeB}$ interface helps to prevent interdiffusion between Ta and CoFeB since TaN constitutes a good diffusion barrier (Rossnagel and Kim, 2003).

Along the same lines, Yang et al. (2014) investigated how the insertion of a thin $\mathrm{TaO}$ layer into the Ta buffer layer affected the PMA of $\mathrm{Ta} / \mathrm{TaOx} / \mathrm{Ta} / \mathrm{CoFeB} / \mathrm{MgO} / \mathrm{W}$ stacks. They observed that at $350{ }^{\circ} \mathrm{C}$ the diffusion of Ta into the $\mathrm{CoFeB}$ could be reduced by the presence of $\mathrm{TaO}$ in the $\mathrm{Ta}$ buffer. This effect can be qualitatively explained by considering the higher heat of the formation of $\mathrm{TaOx}(-1023 \mathrm{~kJ} / \mathrm{Ta} \mathrm{mol})$ than of $\mathrm{MgO}$ $(-602 \mathrm{~kJ} / \mathrm{Mg} \mathrm{mol})$ (Lide, 2000). In the absence of $\mathrm{TaO}$ in the underlayer, Ta atoms tend to diffuse toward the $\mathrm{MgO}$ barrier where they form $\mathrm{TaO}$ along the $\mathrm{MgO}$ interface. When $\mathrm{TaO}$ is already present in the buffer layer, the Ta atoms tend to stay where they are. In addition, the $\mathrm{TaO}$ layer provides some structural rigidity to the stack, hindering the diffusion of Ta.

Several groups investigated the influence of capping layers on anisotropy and damping in $\mathrm{MgO} / \mathrm{CoFeB} /$ capping layer structures (Kubota et al., 2012; Natarajarathinam et al., 2012, Lee et al., 2014). For example, Natarajarathinam et al. (2012) compared the influence of $\mathrm{Ta}, \mathrm{Ru}, \mathrm{MgO}, \mathrm{MgO} / \mathrm{Ru}$, and $\mathrm{V}$ capping layers and found particularly interesting results with $\mathrm{MgO}$ and $\mathrm{V}$ capping layers. Both systems gave a relatively large interfacial anisotropy in the range of $2-2.5 \mathrm{~mJ} / \mathrm{m}^{2}$, and the Gilbert damping for 2-nm CoFeB was significantly lower than with a Ta cap (damping $\alpha \sim 0.004$ after annealing with the $\mathrm{MgO}$ cap and $\sim 0.0065$ with the $\mathrm{V}$ cap as compared to
0.017 for the Ta cap). The case with the $\mathrm{MgO}$ cap will be further detailed as it can take advantage of interfacial PMA at both the $\mathrm{MgO} / \mathrm{CoFeB}$ and $\mathrm{CoFeB} / \mathrm{MgO}$ interfaces [see also Kubota et al. (2012)]. The V cap is of interest for two reasons: first, the reduced Gilbert damping coefficient, which can be explained by the earlier observation by Scheck et al. (2007) that FeV alloys display very low Gilbert damping; second, there is no measurable dead layer at the $\mathrm{CoFeB} / \mathrm{V}$ interface although magnetization of the $\mathrm{CoFeB}$ layer does appear to be significantly reduced in the presence of a $\mathrm{V}$ cap (Natarajarathinam et al., 2012). Lee et al. (2014) investigated the properties of $\mathrm{Nb}$ capping layers and found them to be relatively similar to those of Ta caps.

All these recent studies indicate that significant efforts are ongoing to optimize the growth conditions of these p-MTJs so as to maximize the PMA energy while simultaneously achieving low Gilbert damping and large TMR amplitude.

\section{Influence of the presence of $\mathrm{Mg}$ between $\mathrm{MgO}$ and $\mathrm{CoFeB}$}

Ma et al. (2012) studied how introducing a thin Mg layer (thickness between 0 and $1.0 \mathrm{~nm}$ ) at the interface between $\mathrm{MgO}$ and $\mathrm{CoFeB}$ affected interfacial PMA and the Gilbert damping coefficient. They observed that interfacial anisotropy remains quite high after annealing at temperatures between $250^{\circ} \mathrm{C}$ and $375^{\circ} \mathrm{C}$, even when up to $0.8 \mathrm{~nm}$ of $\mathrm{Mg}$ is introduced at the interface between $\mathrm{MgO}$ and $\mathrm{CoFeB}$. Thus interface anisotropy of $1.46 \mathrm{~mJ} / \mathrm{m}^{2}$ was measured without $\mathrm{Mg}$, while, after annealing at $300{ }^{\circ} \mathrm{C}$ for $1 \mathrm{~h}$, anisotropy of $1.34 \mathrm{~mJ} / \mathrm{m}^{2}$ was measured when $0.6 \mathrm{~nm} \mathrm{Mg}$ was inserted at the interface. If considered in the context of the results presented in Fig. 9 (Manchon et al., 2008), this observation could be explained by the fact that the oxygen from the bulk of the $\mathrm{MgO}$ diffuses toward the $\mathrm{Mg} / \mathrm{CoFeB}$ creating oxygen vacancies in the bulk of the $\mathrm{MgO}$ layer while simultaneously transforming the $\mathrm{Mg} / \mathrm{CoFeB}$ interface into an $\mathrm{MgO} / \mathrm{CoFeB}$ interface. As a result, the interfacial anisotropy does not significantly decrease upon adding $\mathrm{Mg}$. This confirms that the most stable location for the oxygen in the multilayer is at the interface between $\mathrm{MgO}$ and $\mathrm{CoFe}(\mathrm{B})$, as previously observed by XPS and XAS experiments (Manchon et al., 2008). This observation of a slight reduction in anisotropy upon insertion of a $\mathrm{Mg}$ layer is also consistent with ab initio calculations (Yang et al., 2011) showing that the anisotropy is not significantly reduced at underoxidized $\mathrm{Fe} / \mathrm{MgO}$ interfaces, whereas it is strongly reduced at overoxidized $\mathrm{Fe} / \mathrm{MgO}$ interfaces. $\mathrm{Ma}$ et al. also observed that the Gilbert damping coefficient is significantly reduced in $\mathrm{MgO} / \mathrm{Mg} t_{\mathrm{Mg}} / \mathrm{CoFeB} 1.2 \mathrm{~nm} / \mathrm{Ta}$ when $\mathrm{Mg}$ is inserted; dropping from values of around 0.055 without $\mathrm{Mg}$ down to 0.023 with $t_{\mathrm{Mg}}=1 \mathrm{~nm}$. This reduction is interesting from a technological point of view since the critical current for STT switching is proportional to the Gilbert damping.

\section{Influence of CoFeB composition on PMA amplitude and thermal variation}

The composition of the $\mathrm{CoFeB}$ electrodes in contact with the $\mathrm{MgO}$ barrier influences the growth and resulting nanostructure of the p-MTJ stacks and consequently their magnetic and spin-dependent transport properties. 
Fe and bcc Co have very similar lattice parameters at 2.87 and $2.82 \AA$, respectively (Prinz, 1985). To match the bcc $\mathrm{MgO}$ (001) crystallographic lattice, the $\mathrm{Fe}(\mathrm{Co})$ bcc lattice is rotated by $45^{\circ}$ around the (001) direction with respect to that of $\mathrm{MgO}$. This rotation yields relatively good matching between the diagonal of the elementary Fe cell $\sqrt{2} a_{\mathrm{Fe}}=4.06 \AA$ (3.99 $\AA$ for Co), and the lattice parameter for bcc $\mathrm{MgO}, 4.21 \AA$. However, a residual mismatch of $\sim 4 \%$ persists which can generate misfit dislocations in the MgO layer (Dynna et al., 1996). These dislocations can result in a decreased TMR amplitude in $\mathrm{MgO}-$ based MTJs. The mismatch with $\mathrm{MgO}$ is slightly larger for Co than for $\mathrm{Fe}$, but the difference is not very significant. As a result, when considering $\mathrm{CoFeB}$ alloys with various $\mathrm{Co} / \mathrm{Fe}$ concentrations, the influence of the concentration on the mismatch with $\mathrm{MgO}$ can be considered negligible. In contrast, there is an important difference in terms of wetting properties when Fe or Co are grown at the same given temperature on oxides, particularly on $\mathrm{MgO}$. Fe is known to wet much better on oxides (Frank-van der Merwe, i.e., layer-by-layer type of growth, see Fig. 28(a)] than Co does [island growth, see Figs. 28(b) and 28(c)] as illustrated in Fig. 28 (Koch, 1997; Sankar, Dieny, and Berkowitz, 1997; Dieny et al., 1998). This difference in growth modes stems from the difference of surface energy of $\mathrm{Fe}$ and $\mathrm{Co}$ and differences in their bonding energy with $\mathrm{MgO}$.

It therefore seems preferable to use Fe or an Fe-rich alloy in contact with the $\mathrm{MgO}$ barrier to minimize the roughness of the stack and internal stress in the $\mathrm{MgO}$ barrier which could promote dielectric breakdown of the tunnel barrier. Furthermore, a discontinuous magnetic layer has a greater tendency to become superparamagnetic at small thickness (Tsai et al., 2014). Despite these advantages, it has also been shown that $\mathrm{Fe}$ in contact with the barrier, especially when positioned below the barrier, may form an FeO layer which could reduce the TMR amplitude (Meyerheim et al., 2001). As a result, using a moderately Fe-rich $\mathrm{CoFeB}$ layer is preferable below the barrier, but pure Fe is unsuitable. In contrast, an Fe-rich CoFeB layer is more suitable above the barrier.

From magnetic and transport points of view, several important points must be considered and trade-offs must be found. Experimental findings (Sokalski et al., 2012; (a)

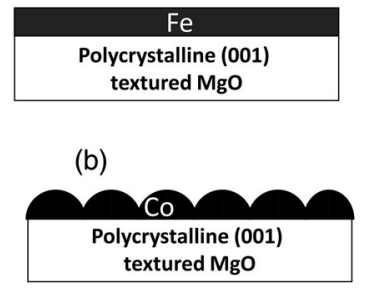

(c)

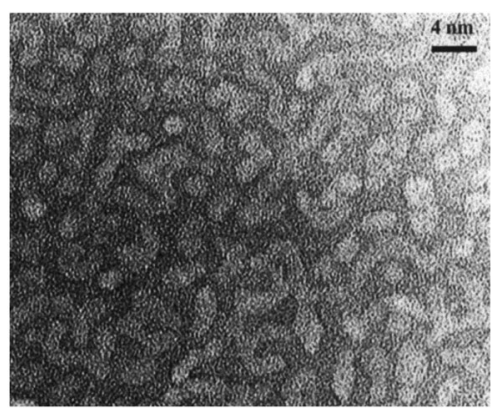

FIG. 28. (a) Schematic representation of layer-by-layer growth of a thin Fe layer on $\mathrm{MgO}$. (b) Schematic representation of the island growth pattern observed for $\mathrm{Co}$ grown on $\mathrm{MgO}$ or other oxides. (c) In-plane transmission electron microscopy view of a thin $(1.6 \mathrm{~nm})$ layer of Co inserted between two $\mathrm{SiO}_{2}$ oxide layers showing how the Co layer is broken into islands. From Dieny et al., 1998.
Devolder, Barisic et al., 2013) confirmed by ab intio calculations (Yang et al., 2011) indicated that the interfacial PMA is significantly larger with $\mathrm{Fe} / \mathrm{MgO}$ than it is with $\mathrm{Co} / \mathrm{MgO}$. For instance, for annealed Ta $3 \mathrm{~nm} / \mathrm{Co}_{20} \mathrm{Fe}_{60} \mathrm{~B}_{20}, 1 \mathrm{~nm} / \mathrm{MgO}$, $2 \mathrm{~nm}$, Devolder, Ducrot et al. (2013) reported an effective anisotropy field of $\mu_{0}\left(H_{k}-M_{s}\right)=430 \pm 10 \mathrm{mT}$ at RT compared to a value of $82 \pm 10 \mathrm{mT}$ for Ta3 nm/ $\mathrm{Co}_{60} \mathrm{Fe}_{20} \mathrm{~B}_{20}$, $1 \mathrm{~nm} / \mathrm{MgO}, 2 \mathrm{~nm}$. Similarly, Yang et al. (2011) calculated an interfacial PMA for a $\mathrm{Fe} / \mathrm{MgO}$ interface of $1.46 \mathrm{~mJ} / \mathrm{m}^{2}$ as compared to $0.19 \mathrm{~mJ} / \mathrm{m}^{2}$ for a pure $\mathrm{Co} / \mathrm{MgO}$ interface. This comparison indicates that, purely from an interfacial PMA point of view, using an Fe-rich $\mathrm{CoFeB}$ alloy next to the interface seems preferable.

However, a second important point related to the magnetic properties of these $\mathrm{CoFeB}$ alloys is their temperature variation. Co has a higher Curie temperature than $\mathrm{Fe}\left(T_{c}^{\mathrm{Co}}=1404 \mathrm{~K}\right.$ vs $\left.T_{c}^{\mathrm{Fe}}=1043 \mathrm{~K}\right)$. This means that compared to the magnetization of $\mathrm{Fe}$, the magnetization of $\mathrm{Co}$ is more resistant to the development of thermal fluctuations as the temperature increases. This has several important consequences in terms of magnetic and transport properties. Because heat-induced magnetic fluctuations grow faster with increasing temperature in Fe-based MTJs than in their Co-based counterparts, a steeper decrease of TMR is expected in $\mathrm{Fe} / \mathrm{MgO} / \mathrm{Fe}$ than in $\mathrm{Co} / \mathrm{MgO} / \mathrm{Co}$ as a function of measurement temperature. This property is indeed observed (Fig. 29). For $\mathrm{Fe} / \mathrm{MgO} / \mathrm{Fe}$ between 4 and $300 \mathrm{~K}$, a $50 \%$ drop in relative TMR value
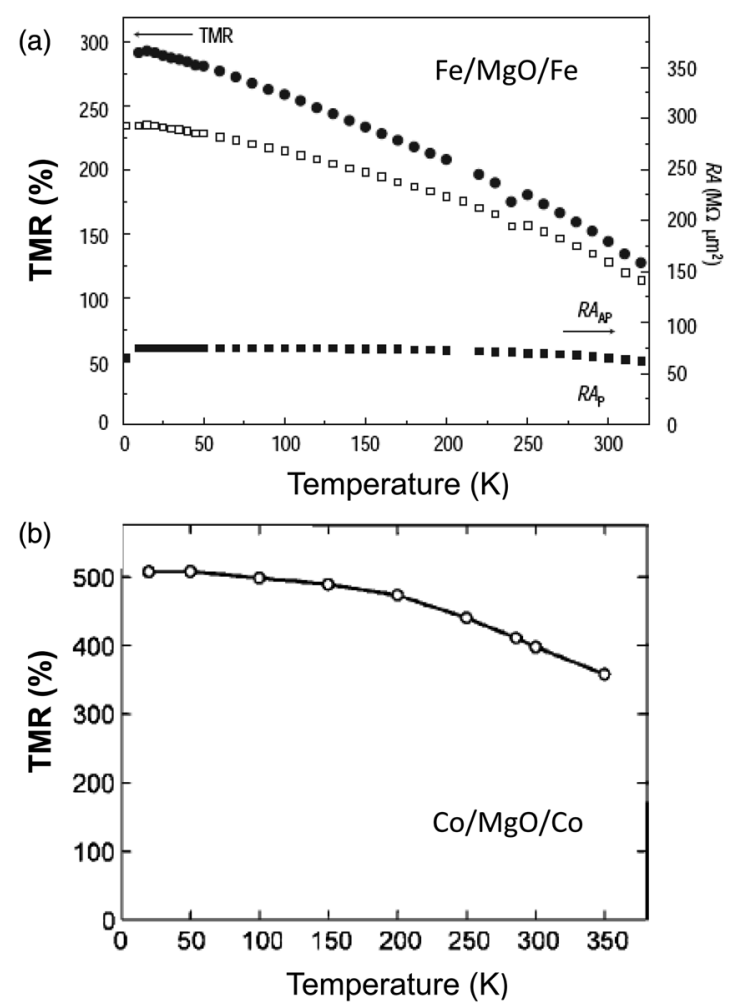

FIG. 29. The variation of TMR as a function of temperature in (a) $\mathrm{Fe} / \mathrm{MgO} / \mathrm{Fe}$. From Parkin et al., 2004. (b) $\mathrm{Co} / \mathrm{MgO} / \mathrm{Co}$. From Yuasa et al., 2006. The three curves in (a) represent the RA product in $P$ (black squares) and $A P$ (white squares) configurations and the resulting TMR (black dots). 


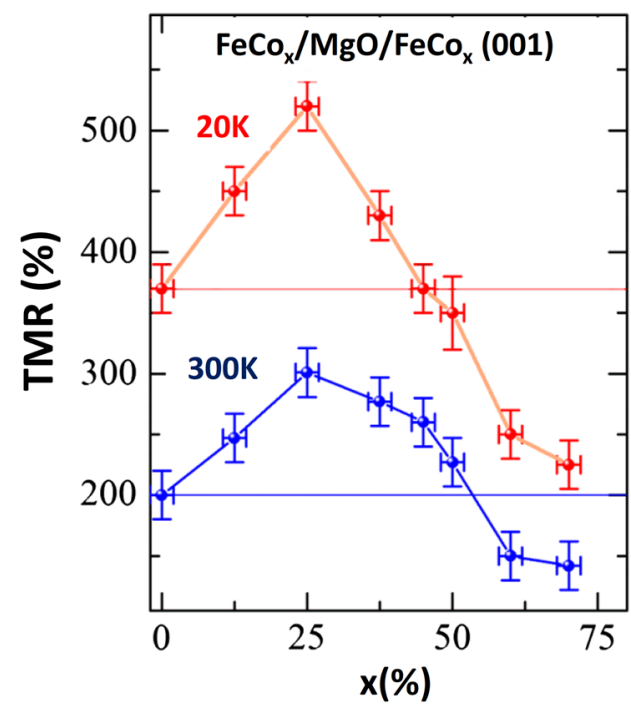

FIG. 30. TMR amplitude in $\mathrm{FeCo}_{x} / \mathrm{MgO} / \mathrm{FeCo}_{x}$-based MTJ vs Co concentration at 20 and $300 \mathrm{~K}$. From Bonell et al., 2012.

is observed (Parkin et al., 2004). In contrast, in $\mathrm{Co} / \mathrm{MgO} / \mathrm{Co}$, TMR drops only by $30 \%$ (Yuasa et al., 2006).

A similar trend is expected for the interfacial PMA. Since the density of thermal fluctuations increases more rapidly with temperature at $\mathrm{Fe} / \mathrm{MgO}$ interfaces than at $\mathrm{Co} / \mathrm{MgO}$ interfaces, this would encourage the use of magnetic electrodes composed of Co-rich alloys rather than Fe-rich alloys. However, as previously mentioned, at least at low temperatures, the anisotropy is much greater with Fe than with Co. A trade-off must therefore be found, and a good compromise seems to be to use $\mathrm{Fe}$ or an Fe-rich alloy such as $\mathrm{Fe}_{75} \mathrm{Co}_{25}$ (which maximizes the TMR amplitude as shown in Fig. 30) (Bonell et al., 2012) right next to the $\mathrm{MgO}$ barrier and a more Co-rich alloy for the bulk of the top magnetic electrode [e.g., $\mathrm{MgO} / \mathrm{Fe} / \mathrm{Co}_{40} \mathrm{Fe}_{40} \mathrm{~B}_{20}$ or $\mathrm{MgO} / \mathrm{Fe}_{75} \mathrm{Co}_{25}, 0.3 \mathrm{~nm} /$ $\mathrm{Co}_{40} \mathrm{Fe}_{40} \mathrm{~B}_{20}$; besides, $\mathrm{Co}_{40} \mathrm{Fe}_{40} \mathrm{~B}_{20}$ has a minimum damping of 0.012 (Devolder, Barisic et al., 2013)].

In summary, the CoFeB composition influences the structural, magnetic, and transport properties of MTJ stacks. A good trade-off in $\mathrm{CoFeB} / \mathrm{MgO} / \mathrm{CoFeB}$ appears to be possible with a composition of the type $\mathrm{Co}_{40} \mathrm{Fe}_{40} \mathrm{~B}_{20} / \mathrm{MgO} / \mathrm{Fe} / \mathrm{Co}_{40} \mathrm{Fe}_{40} \mathrm{~B}_{20}$ which minimizes damping thanks to the $\mathrm{Co}_{40} \mathrm{Fe}_{40} \mathrm{~B}_{20}$ composition and maximizes anisotropy thanks to the interfacial $\mathrm{Fe}$ layer, while also avoiding oxidation of the bottom electrode (Park et al., 2014).

\section{Influence of annealing}

a. Controlling the diffusion of atomic species

To obtain a large TMR amplitude, it is essential to anneal the MTJ stacks ex situ postdeposition, or in situ right after deposition of the $\mathrm{MgO}$ tunnel barrier. This annealing results in crystallization of the $\mathrm{MgO}$ barrier and of the initially amorphous $\mathrm{CoFeB}$ layer. $\mathrm{CoFeB}$ crystallization starts at the $\mathrm{MgO}$ interface and progresses throughout the $\mathrm{CoFeB}$ layer, and during the process B is gradually expelled (Yuasa et al., 2005). Annealing maximizes the interfacial anisotropy at the
$\mathrm{CoFe} / \mathrm{MgO}$ interface, as shown by data presented in Figs. 10 and 11. From a recrystallization point of view, the higher the annealing temperature, the better the expected crystallization. However, excessively high temperature anneals are detrimental because of atomic diffusion of tertiary species toward the $\mathrm{MgO}$ barrier. This is particularly true for structures containing Mn or Ta as illustrated in Fig. 31 (Lee et al., 2006; Ikeda et al., 2008; Karthik et al., 2012). This figure compares the TMR ratios obtained after annealing at various temperatures for four different stacks in which different degrees of Mn or Ta diffusion take place. The purple curve (diamonds) corresponds to an in-plane magnetized MTJ stack comprising a simple pinned layer, exchange biased by an IrMn layer. Stack composition in this case is $\mathrm{Sub} . / \mathrm{Ta}(5) / \mathrm{Ru}(50) /$ $\mathrm{Ta}(5) / \mathrm{NiFe}(5) / \operatorname{IrMn}(8) / \mathrm{CoFe}(2.5) / \mathrm{CoFeB}(0) / \mathrm{MgO}(1.7) /$ $\mathrm{CoFeB}(3) / \mathrm{Ta}(5) / \mathrm{Ru}(15)$. Upon annealing, TMR amplitude increases up to an annealing temperature of $320^{\circ} \mathrm{C}$ (maximum TMR $190 \%$ of initial value). Above this temperature, the TMR amplitude degrades due to the diffusion of Mn from the IrMn layer toward the $\mathrm{MgO}$ barrier where it reduces the spin polarization of the tunneling electrons.

The green curve (squares) corresponds to data for a similar structure, but where the simple pinned layer is replaced by a synthetic antiferromagnetic pinned layer with a $\mathrm{Ru} 2.5 \mathrm{~nm}$ spacer layer between $\mathrm{CoFe}$ and CoFeB.

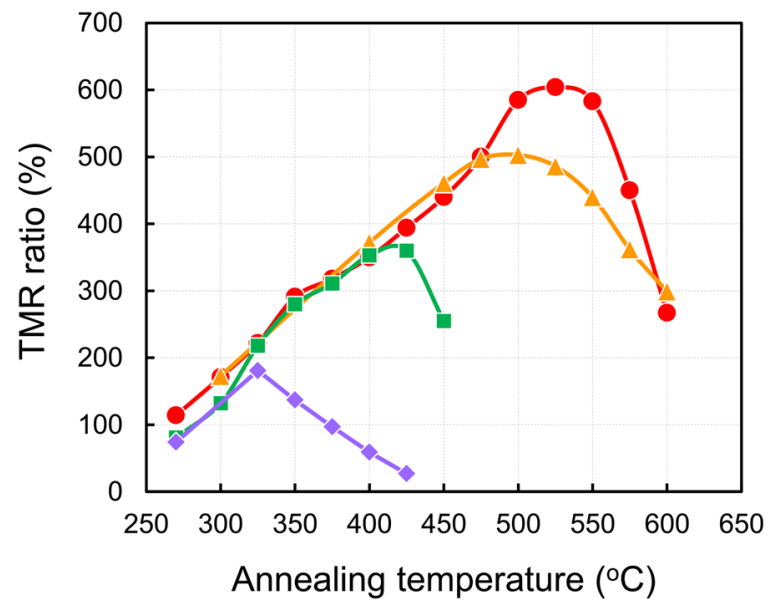

FIG. 31. Influence of annealing temperature on the TMR ratio of CoFeB-MgO MTJs. Purple curve (diamonds): structure comprising a Ru-free simple exchange-biased pinned layer. Composition: Sub./Ta(5)/Ru(50)/Ta(5)/NiFe(5)/IrMn(8)/CoFe(2.5)/ $\mathrm{CoFeB}(3) / \mathrm{MgO}(1.7) / \mathrm{CoFeB}(3) / \mathrm{Ta}(5) / \mathrm{Ru}(15)$. Green curve (squares): structure similar to the previous one but with a synthetic antiferromagnetic pinned layer with a $\mathrm{Ru} 2.5 \mathrm{~nm}$ spacer layer placed between $\mathrm{CoFe}$ and $\mathrm{CoFeB}$. Composition: $\mathrm{Ta}(5) / \mathrm{Ru}(50) / \mathrm{Ta}(5) / \mathrm{NiFe}(5) / \operatorname{IrMn}(8) / \mathrm{CoFe}(2.5) / \mathrm{Ru} 2.5 /$ $\mathrm{CoFeB}(3) / \mathrm{MgO}(1.7) / \mathrm{CoFeB}(3) / \mathrm{Ta}(5) / \mathrm{Ru}(15)$. Orange curve (triangles): double-coercivity MTJ from which the IrMn antiferromagnetic pinning layer was removed. Composition: $\mathrm{Ta}(5) /$ $\mathrm{Ru}(10) / \mathrm{Ta}(5) / \mathrm{CoFeB}(4.3) / \mathrm{MgO}, \quad$ (2.1)/CoFeB(3)/Ta(5)/ $\mathrm{Ru}(5)$. Red curve (circles): Double-coercivity MTJ with thicker magnetic electrodes. Composition: Sub./Ta(5)/Ru(10)/ $\mathrm{Ta}(5) / \mathrm{CoFeB}(6) / \mathrm{MgO}(2.1) / \mathrm{CoFeB}(4) / \mathrm{Ta}(5) / \mathrm{Ru}(5)$. Data derived from Lee et al. (2006), Ikeda et al. (2008), and Karthik et al. (2012). 
In this case, the sample's composition is $\mathrm{Sub} . / \mathrm{Ta}(5) / \mathrm{Ru}(50) /$ $\mathrm{Ta}(5) / \mathrm{NiFe}(5) / \operatorname{IrMn}(8) / \mathrm{CoFe}(2.5) / \mathrm{Ru}(2.5) / \mathrm{CoFeB}(3) /$ $\mathrm{MgO}(1.7) / \mathrm{CoFeB}(3) / \mathrm{Ta}(5) / \mathrm{Ru}(15)$. From earlier work on spin valves, $\mathrm{Ru}$ is known to be a fairly good diffusion barrier for Mn (Huang et al., 2001). Thanks to the reduced Mn diffusion compared to the previous structure, the TMR was found to increase as a function of annealing temperature up to $430{ }^{\circ} \mathrm{C}$, producing a TMR amplitude of up to $360 \%$ of preannealed values. At higher annealing temperatures, the $\mathrm{Ru}$ barrier could no longer prevent Mn diffusion, and TMR once again became degraded.

The orange curve (triangles) corresponds to a double-coercivity MTJ from which the IrMn antiferromagnetic pinning layer was completely removed. The relative orientation of the magnetization in the two magnetic electrodes is controlled only by the difference in coercivity between the two magnetic electrodes. The sample composition is $\mathrm{Sub} . / \mathrm{Ta}(5) / \mathrm{Ru}(10) / \mathrm{Ta}(5) /$ $\mathrm{CoFeB}(4.3) / \mathrm{MgO}(2.1) / \mathrm{CoFeB}(3) / \mathrm{Ta}(5) / \mathrm{Ru}(5)$. These samples no longer contain $\mathrm{Mn}$, so the degradation of TMR above the optimal annealing conditions cannot be ascribed to Mn diffusion. In these samples, the TMR degradation was shown to be linked to Ta diffusion (Ikeda et al., 2008). A maximum TMR of 500\% at RT was obtained after optimal annealing at $500{ }^{\circ} \mathrm{C}$. Higher temperatures trigger Ta diffusion, thus limiting the further rise in TMR amplitude. Some groups reported that $\mathrm{Ta}$ diffusion has already started at $300^{\circ} \mathrm{C}$ (Miyakawa, Worledge, and Kita, 2013; Iihama et al., 2014). Ta tends to diffuse to the $\mathrm{MgO}$ barrier, and since the enthalpy of $\mathrm{Ta}_{2} \mathrm{O}_{5}$ formation $(-244.4 \mathrm{kcal} / \mathrm{mol}$ of $\mathrm{Ta})$ is more negative than that for $\mathrm{MgO}(-143.7 \mathrm{kcal} / \mathrm{mol}$ of $\mathrm{Mg})$, Ta tends to grab oxygen from $\mathrm{MgO}$ so that it will be oxidized into $\mathrm{TaOx}$ while the $\mathrm{MgO}$ is partially reduced into $\mathrm{Mg}$.

In the fourth sample (red curve in Fig. 31, circles), much thicker $\mathrm{CoFeB}$ layers were used to increase the spacing between the Ta buffer or capping layer and the $\mathrm{MgO}$ barrier. The sample composition is $\mathrm{Sub} . / \mathrm{Ta}(5) / \mathrm{Ru}(10) / \mathrm{Ta}(5) / \mathrm{CoFeB}(6) /$ $\mathrm{MgO}(2.1) / \mathrm{CoFeB}(4) / \mathrm{Ta}(5) / \mathrm{Ru}(5)$. This reduces the amount of Ta atoms that reach the $\mathrm{MgO}$ barrier during high temperature annealing. As a result, the TMR amplitude could be increased up to $604 \%$ following an optimized annealing at $520^{\circ} \mathrm{C}$.

Out-of-plane magnetized samples are usually $\mathrm{Mn}$ free since, in principle, no antiferromagnetic materials are required. However, Ta is frequently used as a spacer layer to allow the structural change between $(\mathrm{Pt} / \mathrm{Co})$ fcc structure types and $\mathrm{CoFeB}$ bcc structures within the reference layer, and/or as a B getter material. Therefore Ta diffusion can take place in these structures as in their in-plane magnetized counterparts. Replacing Ta by materials which diffuse less (Mo, W, Hf) may help to increase the optimum annealing temperature. Introducing additional antidiffusion barriers into the stack can also limit interdiffusion, as described for earlier developments on spin valves (Sugita et al., 2001).

\section{b. Influence of irradiation with light ions}

A way to efficiently promote crystallization of the $\mathrm{CoFeB}$ electrodes with a moderate thermal budget consists of assisting a low temperature annealing of the MTJ through light-ion irradiation, typically $15 \mathrm{keV} \mathrm{He}^{+}$irradiation at fluences up to $1.5 \times 10^{19}$ ions $/ \mathrm{m}^{2}$ (Devolder, Barisic et al., 2013). The low interaction cross section, together with the low energy transfer, leads to short-distance displacements and pairwise exchange of atomic positions. Light-ion irradiation has already been shown to promote ordering of $\mathrm{FePt}$ and $\mathrm{FePd}$ L10-ordered alloys at a much lower temperature than without irradiation $\left(300^{\circ} \mathrm{C}\right.$ with irradiation as opposed to $670{ }^{\circ} \mathrm{C}$ without irradiation) (Ravelosona et al., 2000; Bernas et al., 2003). In the case of irradiation of $\mathrm{Ta} / \mathrm{CoFeB} / \mathrm{MgO}$, because $\mathrm{B}$ are the lightest target atoms, they will receive the highest recoiling energy. This can be used to promote atomic mobility of B without generating excessive interdiffusion of the other species present. Some of the main results obtained with this method are illustrated in Fig. 32 (Devolder, Barisic et al., 2013). Two fluence regimes can be identified as follows.

(1) In the low fluence regime (i.e., $F<2 \times 10^{19}$ ions $/ \mathrm{m}^{2}$ ), the effective anisotropy field [Fig. 32(a)] increases in proportion to the irradiation fluence. The Gilbert damping $(\alpha \sim 0.01)$ is almost invariant within the precision of the measurements. The magnetization slightly decreases, but this only partly accounts for the increase in the effective anisotropy: the magnetocrystalline anisotropy is strengthened as these changes occur. These alterations are consistent with

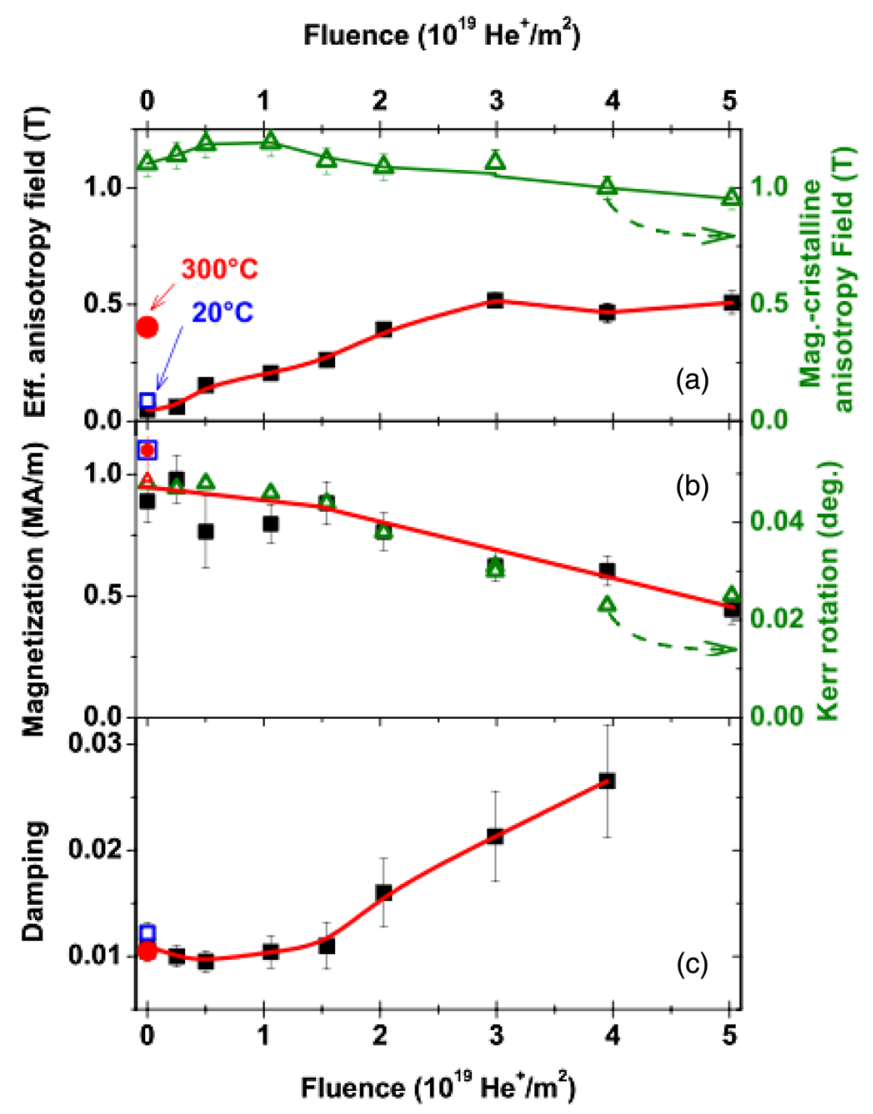

FIG. 32. Evolution of the magnetic properties as a result of annealing at $300^{\circ} \mathrm{C}$ (red circles) or irradiation vs fluence for an initially amorphous $\mathrm{Ta} / \mathrm{Co}_{20} \mathrm{Fe}_{60} \mathrm{~B}_{20}(1 \mathrm{~nm}) / \mathrm{MgO}$ film (empty blue rectangles). The lines are visual guides in all panels. (a) Black squares: Effective anisotropy field, $H_{k}-M_{s}$; green triangles: magnetocrystalline anisotropy field $H_{k}$. (b) Black squares: Magnetization assuming an invariant $\mathrm{CoFeB}$ thickness of $1 \mathrm{~nm}$; green triangles: Kerr rotation at magnetic saturation. (c) Gilbert damping factor. From Devolder, Barisic et al., 2013. 
those generally observed during $\mathrm{CoFeB}$ crystallization at an unaltered abrupt $\mathrm{CoFeB} / \mathrm{MgO}$ interface (Devolder, Barisic et al., 2013).

(2) In the high fluence regime (i.e., $F>2 \times 10^{19}$ ions $/ \mathrm{m}^{2}$ ), the alterations to magnetic properties are quite different. Magnetization is substantially reduced, and the magnetocrystalline anisotropy also decreases. Overall, the effective anisotropy increases before stabilizing. This irradiationinduced increase of effective anisotropy is predominantly due to a decrease in magnetization. The Gilbert damping is substantially increased. These changes are consistent with a high degree of mixing at the $\mathrm{CoFe} / \mathrm{Ta}$ interface and a reduction in the abruptness of the $\mathrm{CoFe} / \mathrm{MgO}$ interface. Indeed, CoFeTa alloys have lower magnetization than $\mathrm{CoFe}$ (Chien et al., 1985; Varga and Doyle, 1996), so interface mixing will naturally decrease the alloy's magnetization. In addition, Ta dopants are known to increase the damping of transition metals (Rantschler et al., 2007).

These results indicate that light-ion irradiation at moderate fluence can assist thermal annealing, thus making $\mathrm{CoFeB}$ crystallization possible and yielding large TMR with a reduced thermal budget compared to thermal annealing alone.

\section{F. Status of STT-MRAM development}

In 2010, the International Technology Roadmap for Semiconductors (ITRS) Emerging Research Devices and Emerging Research Materials Work Groups published a report comparing eight emerging memory technologies representing potential solutions to scale nonvolatile memory technology to and beyond the 16-nm node. These technologies are ferroelectric gate field-effect transistor; nanoelectromechanical switch; STT-MRAM; various types of resistive RAM, in particular, redox RAM; nanothermal phase change RAM; electronic effects RAM; macromolecular memories; and molecular RAM. The report concluded with the statement that "STT-MRAM and redox RAM were identified as emerging memory technologies recommended for accelerated research and development leading to scaling and commercialization of nonvolatile RAM to and beyond the $16 \mathrm{~nm}$ generation" (ITRS, 2010). Since this report was published, the R\&D effort applied to STT-MRAM has been accelerated, and all major microelectronic integrated device manufacturers (IDM) and equipment suppliers are now active in the field. In this section, we report on some published examples of the progress that has been made in the field over the past few years, keeping in mind that the latest developments are often kept confidential.

In 2011, IBM demonstrated that an extremely low bit error rate (BER) could be achieved in STT-MRAM with perpendicular anisotropy (Fig. 33) (Nowak et al., 2011). The BER tests were carried out on 4-Kbit chips. On individual bits, extremely low BER of $10^{-11}$ were achieved with write-voltage pulses lasting $\pm 0.42 \mathrm{~V}-50 \mathrm{~ns}$ or $\pm 0.52 \mathrm{~V}-10 \mathrm{~ns}$. This BER is much better than that achieved with in-plane magnetized STT-MRAM, for which uncontrolled micromagnetic states and backhopping phenomena cause problems (Min et al., 2010). Figure 33 shows BER measurements performed on 256 randomly selected cells, of which only three cells (not shown) were defective. Each of the 253 electrically

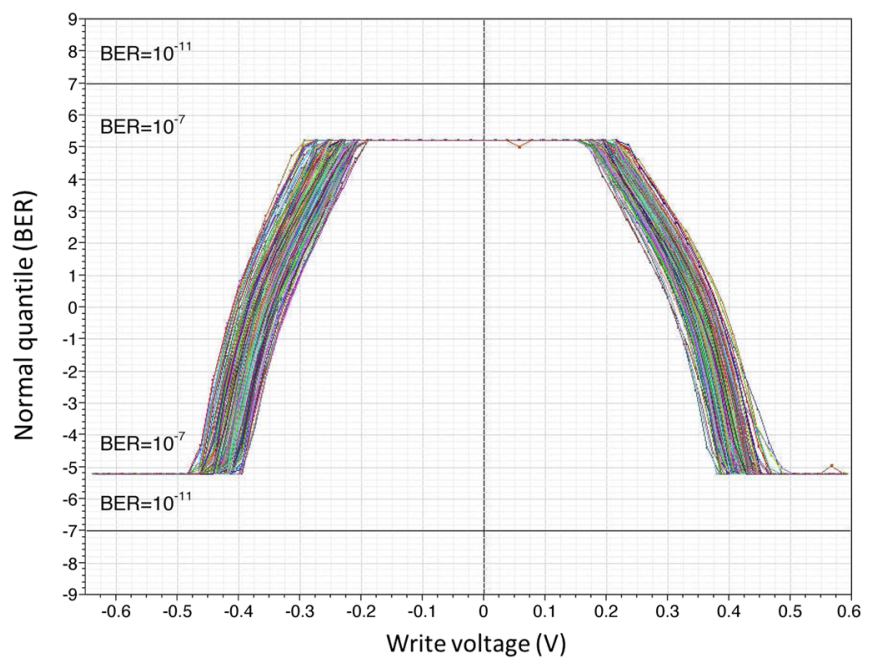

FIG. 33. BER for 253 randomly selected bits. The write pulses lasted $50 \mathrm{~ns}$ and voltage amplitude was varied. From Nowak et al., 2011.

functioning cells attained the stipulated error floor of $10^{-7}$ and operated without errors at several higher voltages, as indicated by the flat parts of the lines in the graphs. Two BER anomalies at around 60 and $560 \mathrm{mV}$ were linked to external noise. Some cell-to-cell variability in write voltage was observed, but its distribution was narrow (typically between 0.38 and $0.5 \mathrm{~V}$ ) and it did not prevent the BER of $10^{-7}$ being achieved. This variability was sufficiently low to allow very good endurance ( $>10^{13}$ cycles) and it was large enough to allow read voltages of around $0.15 \mathrm{~V}$ to be used. The much lower BER measured in out-of-plane magnetized STTMRAM cells compared to in-plane magnetized STTMRAM was very encouraging for further developments in this direction.

The main goal of major IDMs is to replace dynamic random access memory (DRAM) by STT-MRAM at advanced 1x technology nodes. Indeed, as the technology continues to progress beyond the 20-nm node, DRAMs are becoming increasingly difficult to manufacture due to the very high aspect ratio of the capacity contained in each cell. STT-MRAM would be ideal candidates to replace these structures since they can be dense ( $\sim 6 F^{2}$, where $F$ is the minimum feature size that can be realized at the corresponding technology node), fast (write or read cycle 10 ns), have good endurance (number of write cycles $>10^{13}$ ) and, unlike conventional DRAM, they are also nonvolatile, i.e., they can store information without the need for a power supply. Because of these characteristics, significant gains in power consumption, both static and dynamic, are expected when STT-MRAM will be integrated into electronic circuits, such as through the design of normally off or instant on circuits (Kawahara, 2011; Yoda et al., 2012). For DRAM applications, density is a key parameter. Therefore both the MTJ diameter and the selection transistor connected in series with the MTJ must be as small as possible, typically measuring less than $20 \mathrm{~nm}$, and this size should decrease with the technology nodes. Considerable R\&D efforts are therefore focused on the patterning of sub-20-nm p-MTJs. Some representative results of this effort from Samsung electronics were presented at the 2011 International Electron Devices 

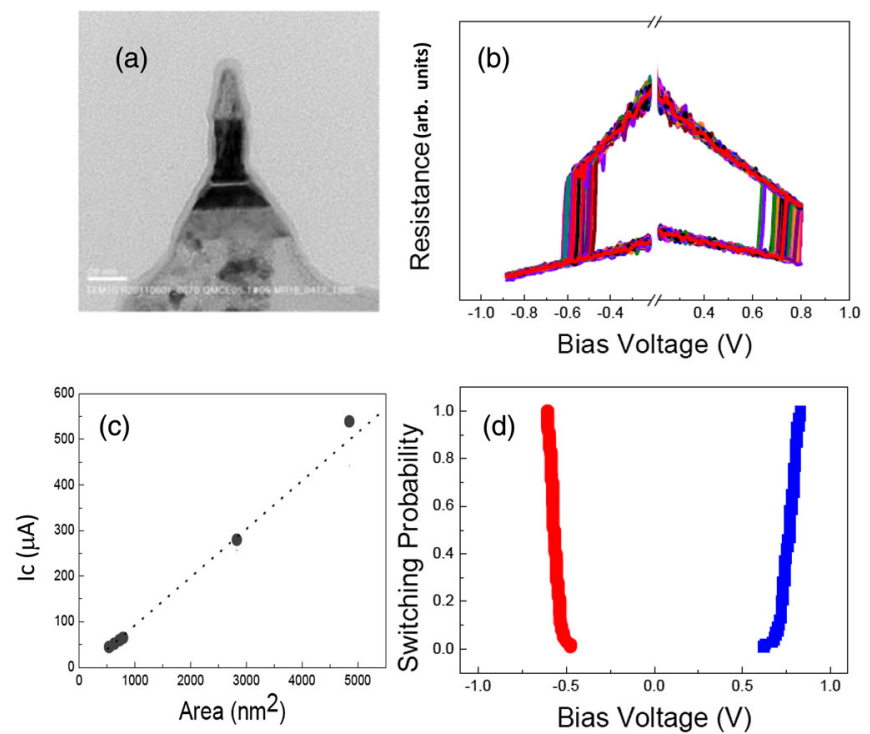

FIG. 34. (a) Cross-sectional transmission electron microscopy (TEM) image of a 17-nm MTJ. The length of the short axis is about $17 \mathrm{~nm}$. (b) STT switching curves for a single cell measured 100 times at a pulse width of $100 \mathrm{~ns}$. (c) Switching current $\left(I_{c}\right)$ as a function of the MTJ cell area at a pulse width of 100 ns. (d) Probability of switching derived from (b). From Kim et al., 2011.

Meeting (IEDM) and are reproduced in Fig. 34 (Kim et al., 2011). This study used out-of-plane magnetized MTJs based on interfacial PMA at a $\mathrm{Co}_{20} \mathrm{Fe}_{60} \mathrm{~B}_{20} / \mathrm{MgO}$ interface. MTJs could be patterned to dimensions as small as $17 \mathrm{~nm}$ [Fig. 34(a)]. The magnetization direction for these cells could be switched by STT [Fig. 34(b)]. Some variability in the switching of each individual cell was observed. This variability was associated with the role of thermal fluctuations which play an important role in triggering the reversal of magnetization through the influence of STT, especially at very small feature sizes. Indeed, since the STT is proportional to the cross product of the storage layer's magnetization by the spin-current polarization (parallel to the magnetization of the reference layer), the magnetization vector for the storage layer must deviate slightly from the normal direction to allow a nonzero STT. This initial deviation angle is stochastically provided by thermal fluctuations, resulting in the distribution plotted in Fig. 34(d). As expected for a first approximation, for a given MTJ stack patterned with different dimensions, the switching current varies in proportion to the area, attaining values of $47 \mu \mathrm{A}$ for $17-\mathrm{nm}$ MTJs in this study. The conclusion of this paper indicated that, while further improvements would be required in terms of the electrical performances of the sub-20-nm MTJ cells if future Gb-density nonvolatile memory systems are to be developed, the capacity to downscale the critical current makes STT-MRAM a promising candidate for the development of nonvolatile memories on this scale.

Further significant progress was made in the past 4 years, in particular, thanks to the use of MTJ stacks where the storage layer is sandwiched between two $\mathrm{MgO}$ tunnel barriers (Rodmacq et al., 2008). Indeed, as the PMA is provided by the $\mathrm{CoFe}(\mathrm{B}) / \mathrm{MgO}$ interface, using double-barrier structures makes it possible to benefit from the two interfaces. The advantages of double-barrier

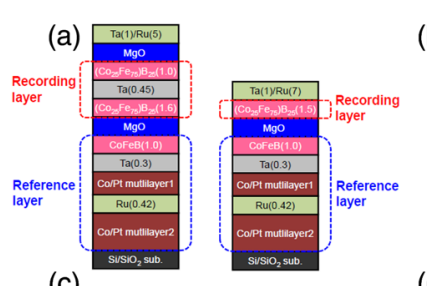

(c)

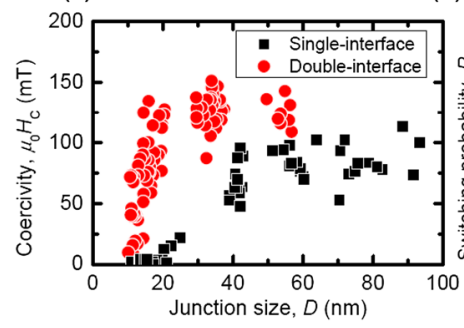

(d)
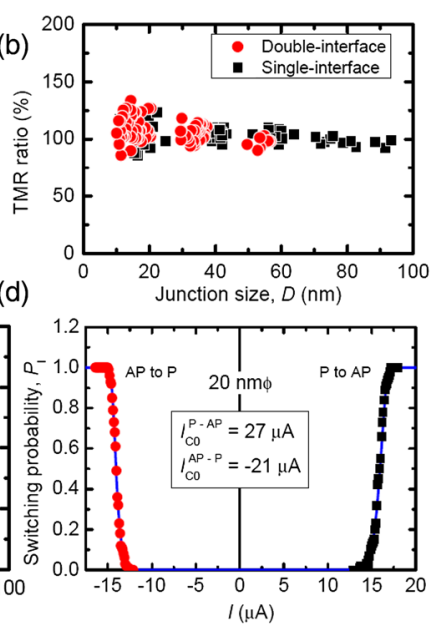

FIG. 35. (a) Stack structures of p-MTJs based on double- and single-CoFeB-MgO interfaces. A synthetic ferromagnetic reference layer is included to reduce the magnetic field produced by the reference layer. (b) TMR for $\mathrm{CoFeB}-\mathrm{MgO}$ MTJ with double and single interfaces as a function of junction size. Size was determined by the resistance of the junctions and the product of resistance and area. (c) Coercivity of $\mathrm{CoFeB}$ with double- and single- $\mathrm{CoFeB} / \mathrm{MgO}$ interfaces as a function of junction size. (d) Switching probability vs current amplitude measured for a 0.1 -s current pulse for 20-nm diameter double interfaces. From Sato et al., 2014.

over single-barrier MTJs are illustrated in Fig. 35 (Sato et al., 2014). In Fig. 35(a), two stacks were compared, a standard MTJ with a single $\mathrm{MgO}$ barrier and a double-barrier stack comprising a $\mathrm{CoFeB} 1.6 \mathrm{~nm} / \mathrm{Ta}, 0.45 \mathrm{~nm} / \mathrm{CoFeB} 1.0 \mathrm{~nm}$ storage layer sandwiched between two $\mathrm{MgO}$ barriers. In this composite storage layer, the intermediate Ta layer is included with the intention of absorbing the $\mathrm{B}$ away from the $\mathrm{MgO}$ interfaces upon annealing and recrystallization of the structure. The resistance area product (RA) for the top $\mathrm{MgO}$ barrier was significantly lower than that for the bottom $\mathrm{MgO}$ barrier, the latter providing the TMR signal. This difference was designed to avoid adding an over-large serial resistance to the stack which would have reduced the effective TMR signal. As a result, the TMR amplitudes obtained with the two types of structure are quite similar [Fig. 35(b)].

The results obtained by Sato et al. also showed that no degradation of the TMR amplitude is observed for the patterned MTJ down to diameters of around $10 \mathrm{~nm}$. This result indicates that the patterning process generates very few edge defects, which by itself is a remarkable result. The benefits of using double-barrier MTJs are clear from Fig. 35(c). The significantly larger coercivity of the doublebarrier stack compared to the single-barrier stack indicates that the thermal stability of the storage layer's magnetization sandwiched between two $\mathrm{MgO}$ barriers is considerably enhanced with a double $\mathrm{MgO}$ barrier. This is especially true for dimensions below $30 \mathrm{~nm}$, and a thermal stability factor of 58 was achieved in 20-nm-diameter double-barrier MTJs. The critical current for switching magnetization in the storage layer was also shown to be reduced in these double-barrier MTJs compared to single-barrier MTJs. This reduction is due to the lower Gilbert damping exhibited by the storage layer in 
the double-barrier MTJs thanks to more limited Ta or $\mathrm{Ru}$ interdiffusion. This lower Gilbert damping for composite storage layers sandwiched between two $\mathrm{MgO}$ barriers was confirmed by Tsunegi et al. (2014) when they measured Gilbert damping for the storage layer and reported values below 0.008 in the presence of an $\mathrm{MgO}$ capping layer. With $\mathrm{Ta}$ capping, the value increased to more than 0.010 . As a result of this reduced Gilbert damping in double-barrier MTJ, Sato et al. obtained a critical current of $24 \mu \mathrm{A}$ in double-barrier MTJs of diameter $20 \mathrm{~nm}$ having a thermal stability factor of 58 .

Along the same lines, TDK-Headway obtained nice results on 8-Mbit STT-MRAM arrays based on double-barrier MTJS (Thomas et al., 2014). Remarkably high thermal stability was obtained for devices with sub-40-nm dimensions, thanks to optimized interface engineering. Thus, STT efficiency greater than $5 k_{B} T / \mu \mathrm{A}$ and energy barriers exceeding $100 k_{B} T$ at RT were reported for sub-40-nm diameter devices for which TMR was larger than $150 \%$. This team also demonstrated that the stacks developed were thermally stable up to $400{ }^{\circ} \mathrm{C}$; thus they exceeded the requirements for $\mathrm{Si}$ CMOS back-end processing paving the way toward the realm of nonvolatile embedded memory in STT-MRAM technology (Thomas et al., 2014).

Even more recently, Hynix/Toshiba reported a 4-Gbit STTMRAM chip at IEDM 2016 demonstrating that a route toward high-density STT-MRAM is possible (Chung et al., 2016).

In the double-barrier MTJs discussed earlier, the top electrode above the upper $\mathrm{MgO}$ barrier is nonmagnetic; it therefore has no STT or tunnel magnetoresistance influence on the storage layer. Ultimately, the performances of doublebarrier p-MTJ could be further optimized by adding a second pinned polarizer on top of the second $\mathrm{MgO}$ barrier. This addition would increase the STT's efficiency by taking advantage of the STT contributions from both the bottom and top pinned layers (Fig. 36). Depending on whether the storage layer is chosen as a composite ferromagnetic layer [Fig. 36(a)] or a synthetic antiferromagnetic layer [Fig. 36(b)], the magnetic alignment of the two polarizing layers should be in antiparallel or parallel configuration, respectively. The idea that the cumulative influence of STT from top and bottom polarizing layers could be exploited in these types of structure was first proposed by Dieny and Redon (2001); see Fig. 8 and associated discussion. If, for instance, the case of a ferromagnetic storage layer is considered [Fig. 36(a)], when

(a)

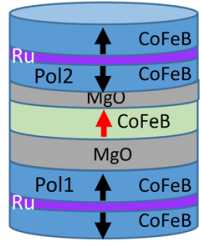

« $1 »$

(b)

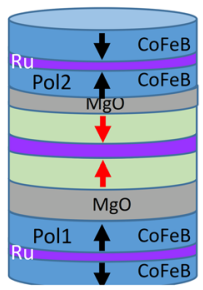

Synthetic antiferromagnetic storage layer

« $1 »$

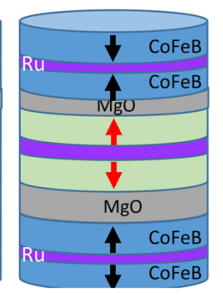

Ferromagnetic storage layer

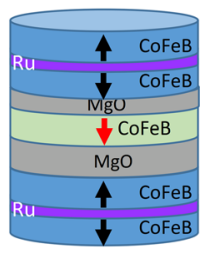

electrons flow from the bottom to the top of the structure, the electrons tunneling from the bottom polarizer [Pol1 in Fig. 36(a)] will exert a direct STT influence on the magnetization of the storage layer [Ferro Sto in Fig. 36(a)] which tends to align parallel to that of Pol1. In addition, electrons for which the spin is opposite to the magnetization of Pol2 will accumulate in the storage layer because it is more difficult for them to tunnel through the top barrier than electrons with spin parallel to Pol2 magnetization. This spin accumulation also generates an STT influence on the storage layer's magnetization, which tends to align antiparallel to Pol2 magnetization, i.e., parallel to Pol1 magnetization. Therefore, both STT contributions will tend to favor the same state, thus increasing the STT efficiency. This increased efficiency will also be observed if the electrons flow from top to bottom. Such structures have recently been grown and studied at the wafer (Cuchet et al., 2015) and pillar levels (Hu et al., 2015). Thanks to an appropriate adjustment of the coercivity of the bottom and top electrodes, they were able to reach the desired magnetic configurations to take advantage of the enhanced STT efficiency.

In these structures, a slight reduction in TMR amplitude might be expected due to the opposing configurations across the bottom and top $\mathrm{MgO}$ barriers: when one is in parallel configuration, the other is antiparallel, and vice versa. Therefore the two TMR contributions for the two MgO barriers will be subtracted one from the other. However, if the RA for the two barriers is chosen to be sufficiently different (for instance a factor of 10), the TMR reduction can be minimized (only $10 \%$ in relative value). This reduction is relatively minor compared to the doubled in STT efficiency obtained.

In conclusion, rapid progress is being made in the development of STT-MRAM technology thanks to the involvement of major microelectronics stakeholders and equipment suppliers. The remaining challenges concern the dot-to-dot variability for high-density memory products (replacement of DRAM) and resistance to electrical breakdown for very fast memories (SRAM replacement). Embedded products with larger MTJ size $(\sim 40 \mathrm{~nm})$ are expected to reach the market soon, then a SRAM type of applications, to be gradually followed by standalone high-density products as the technology matures.

\section{VOLTAGE CONTROL OF THE ANISOTROPY AND APPLICATIONS IN LOW-POWER MAGNETIC MEMORIES}

In this section we discuss a new phenomenon emerging from the main topic of this review, namely, the possibility to modify the PMA at transition metal/oxide interfaces by applying an electric field. This phenomenon often called voltage control of magnetic anisotropy (VCMA) has attracted considerable interest from both scientists and engineers since it represents a viable alternative to energy-demanding magnetic field- and/or STT-controlled magnetization switching in spintronic devices and paves the way to the latter with ultralow-power consumption [see, for example, Wang, Lee, and Amiri (2015) and Lin et al. (2016)].

In the first experimental report by Weisheit et al. (2007), ordered iron-platinum (FePt) and iron-palladium (FePd) intermetallic compounds immersed in an electrolyte were used. The results demonstrated that the MCA can be
FIG. 36. Schematic representation of double-barrier MTJ stacks with (a) single ferromagnetic and (b) synthetic antiferromagnetic storage layers. 
reversibly modified by an applied electric field. This influence of an electric field on MCA in thin ferromagnetic films (Duan et al., 2008; Nakamura et al., 2009a, 2009b) and metallic slabs (Tsujikawa and Oda, 2009) is supported by first-principles calculations predicting several tens of $\mu \mathrm{J} / \mathrm{m}^{2}$ change of MAE for $1 \mathrm{~V} / \mathrm{nm}$ electric field variation. Figure 37 shows this level of variation for $\mathrm{Pt} / \mathrm{Fe} / \mathrm{Pt}, \mathrm{Fe} / \mathrm{Pt}$, and $\mathrm{Fe}$ slabs. Since the MAE dependency on applied electric field was found to be almost linear, it is convenient to characterize this phenomenon based on the slope value, or electric field control coefficient of interfacial magnetocrystalline anisotropy. This value is often noted $\beta$ and is expressed in energy per voltage per length units. It is important to note that, according to the slope and
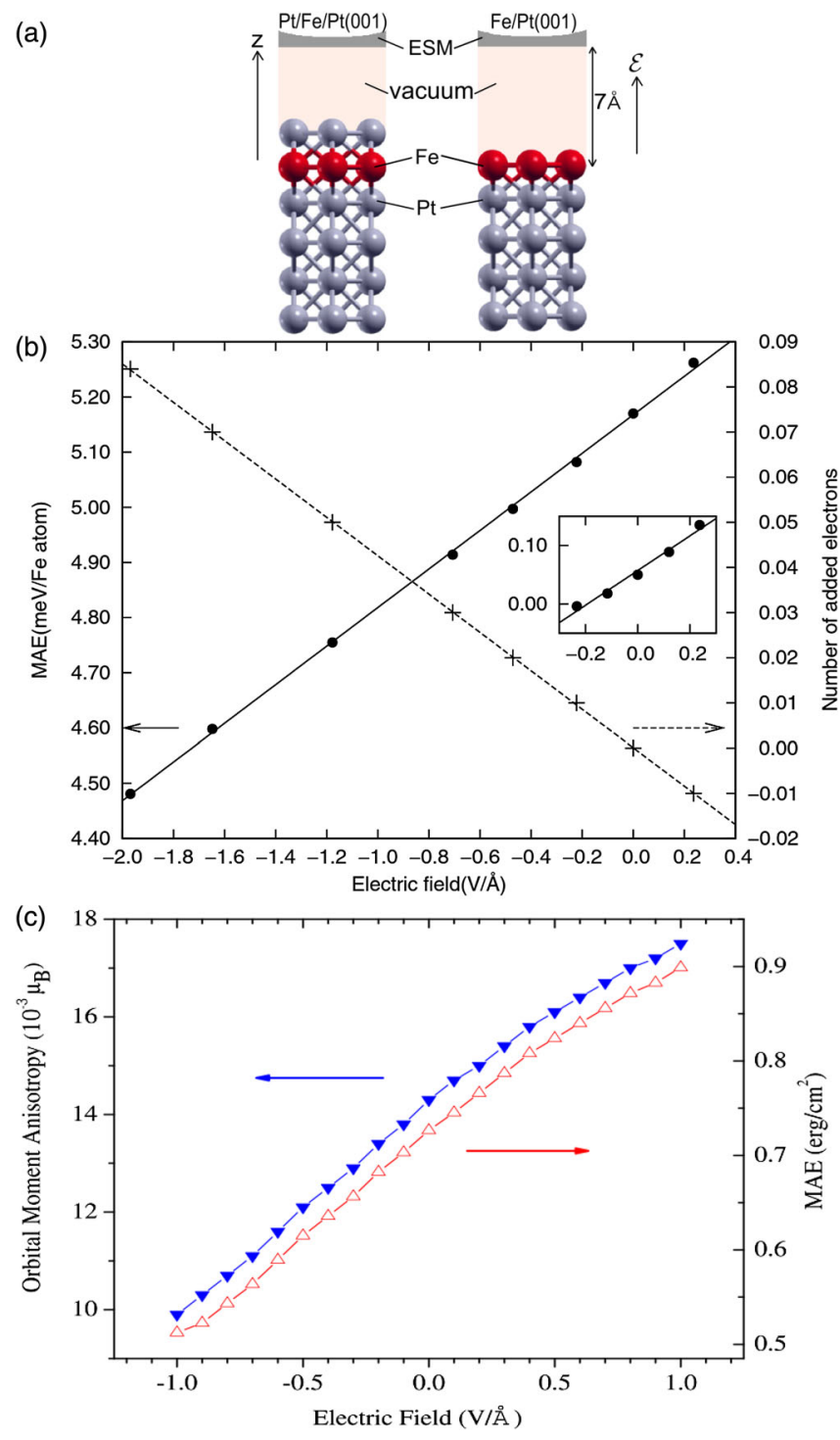

FIG. 37. (a) Schematic representation of the computational models and (b) how MAE (solid dots) and the number of electrons added (crosses) depend on the electric field for $\mathrm{Pt} / \mathrm{Fe} / \mathrm{Pt}(001)$. The inset shows the MAEs as a function of the electric field for $\mathrm{Fe} / \mathrm{Pt}(001)$. From Tsujikawa and Oda, 2009. (c) Electric-field-induced changes in calculated orbital moment anisotropy for surface $\mathrm{Fe}$ atoms and surface magnetocrystalline anisotropy energy (MAE) for a 15-ML-thick Fe (001) slab. From Duan et al., 2008. convention used in these reports, decreasing the number of electrons at the interface by altering the applied electric field promotes the appearance of PMA.

Major attention has been paid to VCMA at $\mathrm{FM} / \mathrm{MgO}$ interfaces and in MTJ, both experimentally (Shiota et al., 2009, 2012; Endo et al., 2010; Nozaki et al., 2010; Seki et al., 2011; Wang et al., 2012) and theoretically (Nakamura et al., 2010; Niranjan et al., 2010; Shimabukuro et al., 2010; Tsujikawa et al., 2011; Suzuki et al., 2013). This is the start of voltage-controlled anisotropy switching in MTJs for the development of low-power memory and logic devices. Maruyama et al. (2009) showed that a relatively small electric field $(\sim 100 \mathrm{mV} / \mathrm{nm})$ applied across an $\mathrm{Au} / \mathrm{Fe} / \mathrm{MgO} /$ polyimide/ITO structure prepared by molecular beam epitaxy can cause a significant change ( $40 \%)$ in the PMA of a bcc $\mathrm{Fe}(001) / \mathrm{MgO}(001)$ junction. This anisotropy is estimated at about $8.4 \mu \mathrm{J} / \mathrm{m}^{2}$ and corresponds to the extrapolated value of the MAE change $\sim 90 \mu \mathrm{J} / \mathrm{m}^{2}$ by $1 \mathrm{~V} / \mathrm{nm}$. The same group went on to perform voltage-induced magnetization switching with magnetic field assistance in a similar structure where $\mathrm{Fe}_{80} \mathrm{Co}_{20}$ was substituted for $\mathrm{Fe}$; the thickness of the magnetic layer was carefully optimized (Shiota et al., 2009). The magnetization switching was demonstrated using polar-Kerr hysteresis curves with a 0.58 -nm-thick $\mathrm{Fe}_{80} \mathrm{Co}_{20}$ layer and

(a)

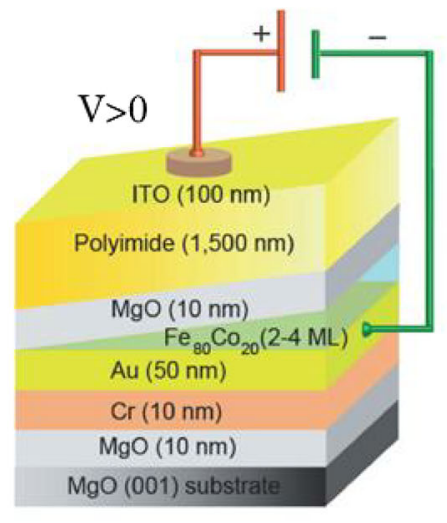

(b)

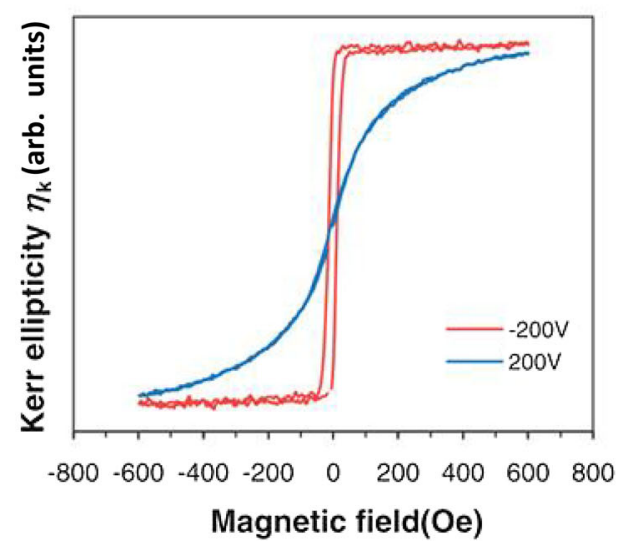

FIG. 38. (a) The sample structure used to investigate voltageinduced magnetization switching. (b) Magnetic hysteresis curves for a 0.58 -nm-thick $\mathrm{Fe}_{80} \mathrm{Co}_{20}$ layer, measured under positive (blue, S-shaped curve) and negative (red, step-shaped curve) bias voltage applications. From Shiota et al., 2009. 
applying positive and negative bias voltages, as shown in Fig. 38. To observe the same magnitude of effect in a magnetic tunnel junction configuration, Nozaki et al. (2010) replaced polyimide/ITO by $\mathrm{MgO} / \mathrm{Fe} / \mathrm{Au}$ and prepared $\mathrm{Au} / \mathrm{Fe}_{80} \mathrm{Co}_{20} /$ $\mathrm{MgO} / \mathrm{Fe} / \mathrm{Au}$ MTJs. An estimated surface MAE change of $\sim 15 \mu \mathrm{J} / \mathrm{m}^{2}$ for $0.4 \mathrm{~V} / \mathrm{nm}$ applied (which extrapolates to $37.5 \mu \mathrm{J} / \mathrm{m}^{2}$ for $1 \mathrm{~V} / \mathrm{nm}$ ) was reported. This change is lower than that observed with samples containing polyimide. A possible explanation for this difference was the influence of charges trapped at the $\mathrm{MgO} /$ polyimide interface (Maruyama et al., 2009; Nozaki et al., 2010).

Finally, a significant breakthrough was reported by the same group using similar MTJ configurations (Shiota et al., 2012). In this series of experiments, Shiota et al. (2012) demonstrated coherent precessional magnetization switching when electric field pulses were applied to induce bistable toggle switching. Importantly, the estimated power consumption for single switching was found to be 500 times smaller than that required for a spin-current-injection (STT) switching process.

Despite these results, it is known that industrial applications prefer to use sputtered devices rather than epitaxial ones. Much attention therefore has also been paid to the influence of the electric field on MAE behavior in structures prepared by sputtering as these structures are much more attractive for spintronic applications. Thus, Endo et al. (2010) investigated how an electric field affected thickness-dependent $\mathrm{MA}$ in $\mathrm{MgO} / \mathrm{Co}_{40} \mathrm{Fe}_{40} \mathrm{~B}_{20} / \mathrm{Ta}$ structures. They found almost linear dependence of MAE on the electric field (see Fig. 39), with a change of $-23 \mu \mathrm{J} / \mathrm{m}^{2}$ for $7.0 \mathrm{MV} / \mathrm{cm}$, which corresponds to $\sim 33 \mu \mathrm{J} / \mathrm{m}^{2}$ for $1 \mathrm{~V} / \mathrm{nm}$ (Endo et al., 2010). A purely electrical manipulation of the MA of a sputtered $\mathrm{Co}_{40} \mathrm{Fe}_{40} \mathrm{~B}_{20}$ film with a clear transition from in-plane to out-of-plane anisotropy was achieved by applying relatively low voltages across the $\mathrm{CoFeB} /$ oxide stacks (Kita et al., 2012). Similarly, a groundbreaking experiment was carried out by Wang et al. (2012) demonstrating that both the magnitude and direction of an electric field could directly affect the PMA of the CoFeB layers in $\mathrm{CoFeB} / \mathrm{MgO} / \mathrm{CoFeB}$ p-MTJs, such that the magnetic configuration could be switched at much smaller current densities than by STT. The change to perpendicular anisotropy energy is estimated to be $\sim 50 \mu \mathrm{J} / \mathrm{m}^{2}$ for $1 \mathrm{~V} / \mathrm{nm}$ of applied electric field (Wang et al., 2012).

Since $L 1_{0}$-ordered Pt- and Pd-based alloys represent important hard magnetic materials for spintronics due to their

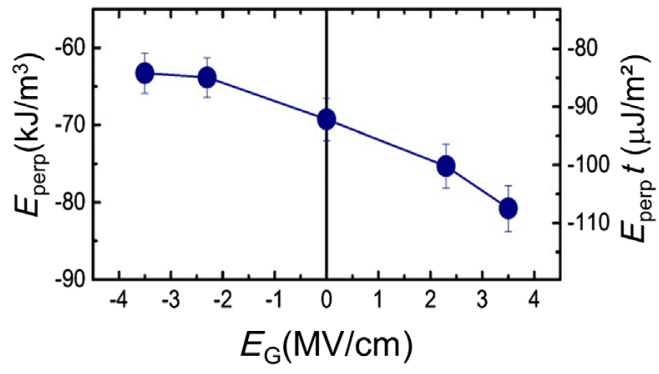

FIG. 39. Magnetic anisotropy energy for devices with a $\mathrm{MgO} / \mathrm{Co}_{40} \mathrm{Fe}_{40} \mathrm{~B}_{20} / \mathrm{Ta}$ structure vs applied electrical field. From Endo et al., 2010. strong uniaxial anisotropy, they have also been used to explore MCA electric-field-control properties in devices where they are interfaced with $\mathrm{MgO}$. Seki et al. (2011) investigated the effect of voltage on the magnetic energy of perpendicularly magnetized FePt layers in $\mathrm{Au} / \mathrm{FePt} / \mathrm{MgO} / \mathrm{AlOx}$ devices and clarified the role played by an $\mathrm{MgO}$ insulating layer on the thin FePt layer. The electric-field effect on magnetic energy for $\mathrm{FePt} / \mathrm{MgO} / \mathrm{AlOx}$ was estimated to be $18.6 \mathrm{fJ} /(\mathrm{Vm})$. They concluded their study by comparing results for devices with and without an $\mathrm{MgO}$ layer (i.e., FePt/MgO/AlOx vs $\mathrm{FePt} / \mathrm{AlOx}$ ) and indicated that the voltage effect is quite sensitive to the surface condition of the FePt layer. Notably, Zhu et al. (2014) used first principles to calculate the electric field dependence of MAE for $\mathrm{Pt} / \mathrm{FePt} / \mathrm{MgO}$ structures comprising $L 1_{0}$-ordered FePt layers (Fig. 40).

It is interesting to note that in almost all these reports on epitaxial and sputtered polycrystalline structures the electricfield-control coefficient values were estimated to be between $\sim 10$ and $\sim 50 \mathrm{fJ} /(\mathrm{V} \mathrm{m})$ and rarely exceeded this range. These results are in reasonably good agreement with theoretical data obtained using first-principles calculations for structures both with and without MgO (Duan et al., 2008; Nakamura et al., 2009a, 2010; Niranjan et al., 2010; Shimabukuro et al., 2010; Zhu et al., 2014; Ibrahim et al., 2016b). This phenomenon is widely accepted to be induced by electric field modification of the electronic orbitals of interfacial atoms. In particular, the decrease (increase) in electronic charge at the $\mathrm{FM} / \mathrm{MgO}$ interface tends to enhance the PMA (IMA), as observed both experimentally and theoretically, as for examples in $\mathrm{Au} / \mathrm{Co}(\mathrm{Fe}) / \mathrm{MgO}$ and $\mathrm{Pt} / \mathrm{Fe} / \mathrm{MgO}$ structures (Nakamura et al., 2010; Tsujikawa et al., 2011; Suzuki et al., 2013). It is interesting to note that the intrinsic electric dipole exists at the $\mathrm{Fe} / \mathrm{MgO}$ interface (Ibrahim et al., 2016b) in addition to that induced by an applied electrical field (Nakamura et al., 2009b). The dipole electric field amplitude is correlated with the ionic positions of the $\mathrm{O}$ and $\mathrm{Mg}$ ions and directly influences the interfacial PMA. As a result there is a coupling between the structural, electrical, and magnetic properties of

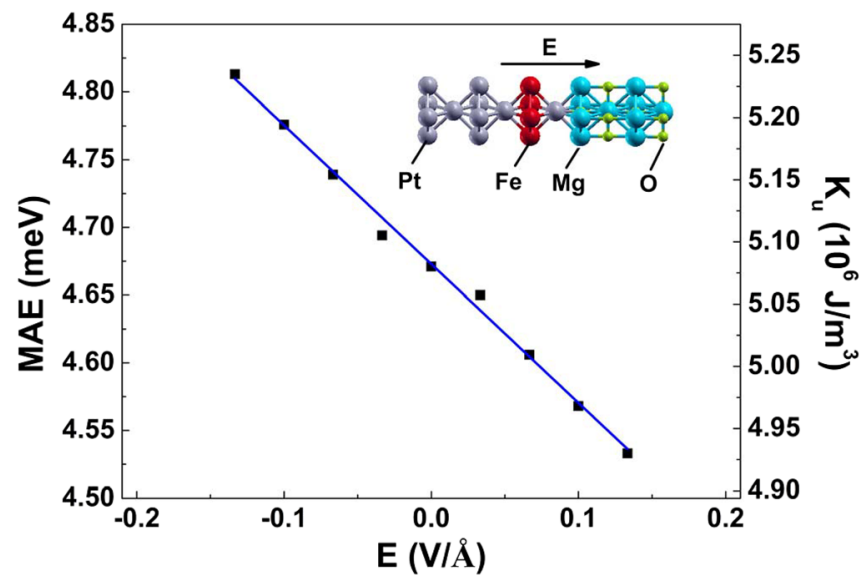

FIG. 40. First-principles calculation of the relationship between MAE and applied electric field as measured for $\mathrm{Pt} / \mathrm{FePt} / \mathrm{MgO}$ structures. The solid line is a linear fit to the calculated data. The inset shows the structure of the model $\mathrm{MgO} / \mathrm{FePt} / \mathrm{Pt}(001)$ film and the negative direction of the electric field applied is indicated by the black arrow. From Zhu et al., 2014. 
(a)

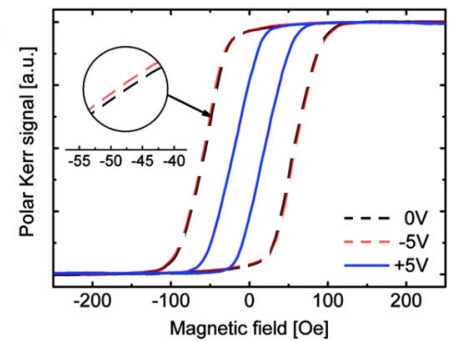

(c)

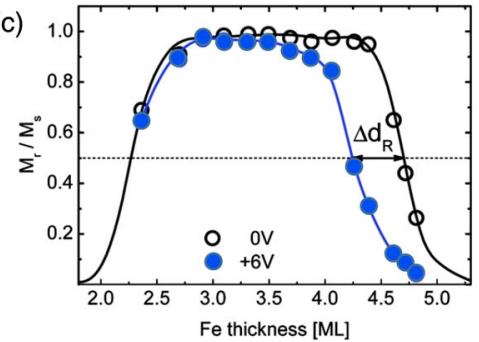

(b)
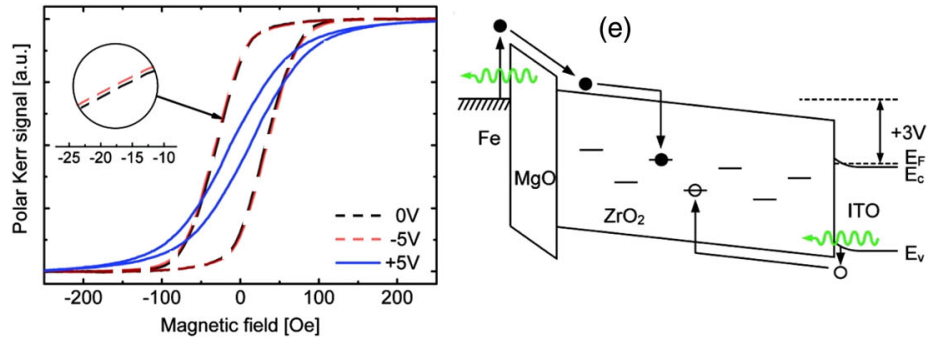

(d)
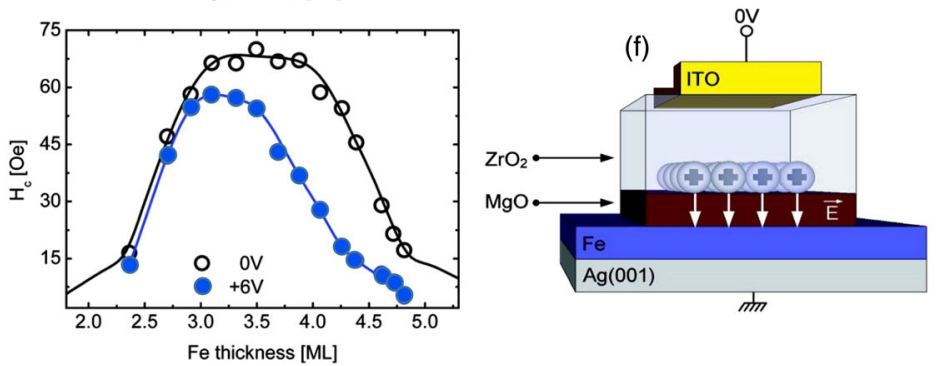

FIG. 41. Polar MOKE hysteresis loops measured while applying an external bias voltage of $0,-5$, and $+5 \mathrm{~V}$ at (a) $4.3 \mathrm{ML}$ Fe and (b) 4.6 ML Fe. Thickness dependence of (c) remanent to saturation magnetization ratio and (d) the coercive field on a continuous Fe slab (black line) or discrete ITO electrodes at a bias voltage of $0 \mathrm{~V}$ (black circle) or $+6 \mathrm{~V}$ (solid blue circle). (e) Schematic band structure of the device studied at $+3 \mathrm{~V}$ gate bias illustrating two possibilities for optically assisted charge injection into the $\mathrm{ZrO}_{2}$ charge-trapping layer which could explain the dependencies seen in (a) and (b). (f) Schematic representation of the device with trapped holes in $\mathrm{ZrO}_{2}$ generating an internal electric field across the $\mathrm{MgO}$ barrier. From Bauer et al., 2012.

the $\mathrm{Fe} / \mathrm{MgO}$ interface so that the latter can be considered as a "multiferroic interface."

Considerable attention has been paid to the impact of interfacial oxidation conditions on electric-field-control properties of magnetocrystalline anisotropy (Nakamura et al., 2010; Niranjan et al., 2010). For instance, Nakamura et al. (2010) indicated that an $\mathrm{FeO}$ layer at the $\mathrm{Fe} / \mathrm{MgO}$ interface plays a crucial role in determining electric field switching properties of MCA. They also highlighted the importance of electric-field-induced displacement of interfacial Fe atoms. Others drew similar conclusions on the importance of the structural relaxation of the Co-O interatomic distances in $\mathrm{Au} / \mathrm{Co} / \mathrm{MgO}$ systems (Suzuki et al., 2013). The slope values, however, remained in the same typical range of magnitudeseveral tens of fJ/(V m) (Nakamura et al., 2010; Suzuki et al., 2013). Therefore, so far, the mechanism of electric-field control of MCA can be considered to be based only on its influence on electronic occupation of orbitals, or alternatively on rigid band model considerations, which explains why slope values remain well below $\sim 100 \mathrm{fJ} /(\mathrm{V} \mathrm{m})$.

A novel era in electric-field control of MCA has recently emerged which allows the electric field coefficient to be increased by at least 1 or 2 orders of magnitude. This novelty is based on ionic electromigration phenomena, or trapped charges. Among the first indications of the existence of such phenomena, we cite several reports by Suzuki's group, including the study based on an $\mathrm{Au} / \mathrm{Fe}_{80} \mathrm{Co}_{20} / \mathrm{MgO} /$ polyimide/ITO structure where, for certain FeCo layer thicknesses, PMA changes corresponding to electric field slope values up to more than $800 \mathrm{fJ} /(\mathrm{Vm})$ were measured (Ha et al., 2010). Furthermore, the same group reported unusually large electric-field-induced PMA sensitivity of $L 1_{0}$-ordered $\mathrm{FePd}$ thin films interfaced with $\mathrm{MgO}$ in epitaxial $\mathrm{Pd} / \mathrm{Fe}_{0.5} \mathrm{Pd}_{0.5} / \mathrm{MgO}$ multilayers deposited on $\mathrm{MgO}$ (Bonell et al., 2011). In these systems, they reported a slope value of $\sim 600 \mathrm{fJ} /(\mathrm{V} \mathrm{m})$ (Bonell et al., 2011). Next, Bauer et al. (2012) proposed a magnetoelectric charge-trap memory design based on an $\mathrm{Fe} / \mathrm{MgO} / \mathrm{ZrO}_{2}$ structure and reported what was, at the time, the largest voltage-induced change to surface MA for a metallic thin film $[\sim 944 \mathrm{fJ} /(\mathrm{V} \mathrm{m})]$. They pointed out that the external bias alone due to the electric field cannot directly account for the magnetoelectric effect observed, and they directly correlated this change with the charge density trapped in an adjacent charge-storage layer (Bauer et al., 2012). Figure 41 shows schematic representations and the magnetic properties measured for the proposed magnetoelectric chargetrap heterostructure device. This device provides a natural interface between conventional electronic and magnetic storage and logic devices while also providing a viable alternative

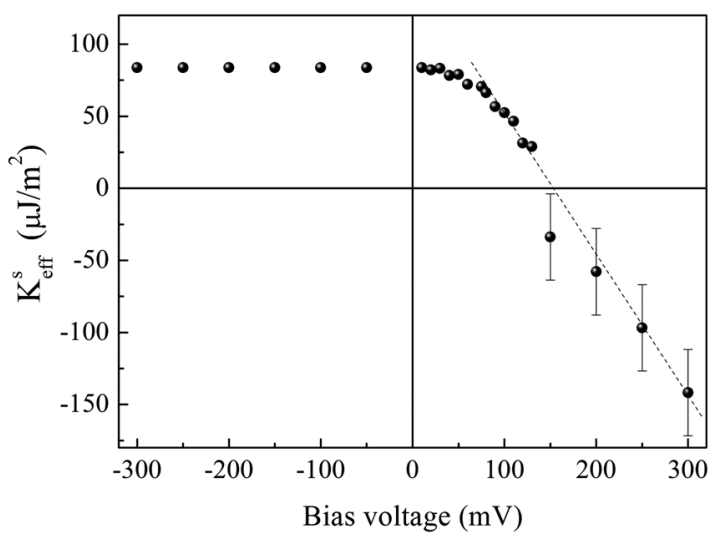

FIG. 42. Effective surface anisotropy as a function of the bias voltage applied to a $\mathrm{V} / \mathrm{Fe} / \mathrm{MgO} / \mathrm{Fe} \mathrm{MTJ}$. The dashed line is a visual guide and was used to determine the $\beta$ parameter. From Rajanikanth et al., 2013. 
to more cumbersome designs such as those based on ferroelectric or ferromagnetic stacks [see Bauer et al. (2012) for details].

Bonell et al. (2013) investigated the influence of an electric field on an ultrathin FeCo film in $\mathrm{Au} / \mathrm{Fe}_{0.9} \mathrm{Co}_{0.1} / \mathrm{MgO}$ structures using XAS spectroscopy and magnetic circular dichroism. Even though they were unable to quantify the MCA sensitivity, they did report partial oxidation of Fe during the microfabrication. Furthermore, it was found that the oxidation state could be reversibly controlled by an electric field, thus electrochemical phenomena influence the magnetism measured at FM/Ox interfaces (Bonell et al., 2013). Another significant advance was reported by Rajanikanth et al. (2013) who investigated the influence of an electric field on the interface $\mathrm{MA}$ at $\mathrm{Fe} / \mathrm{MgO}$ (a)

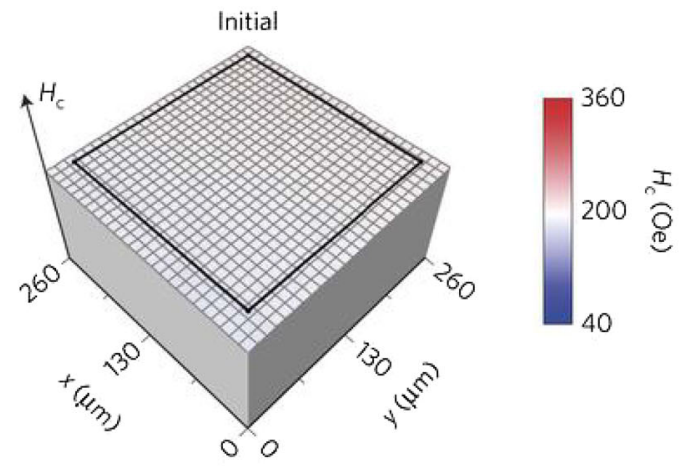

(c)

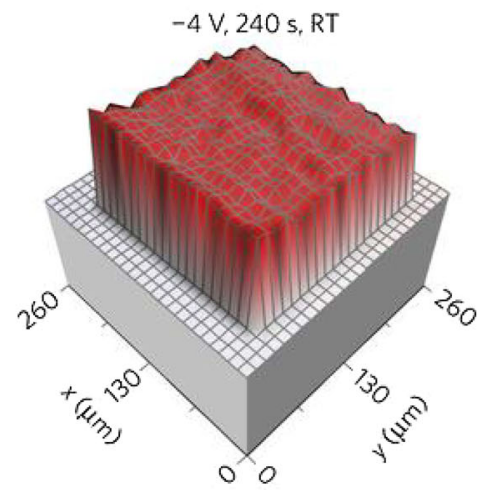

(e)

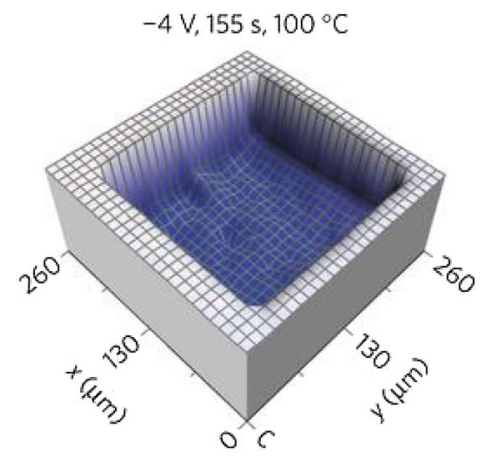

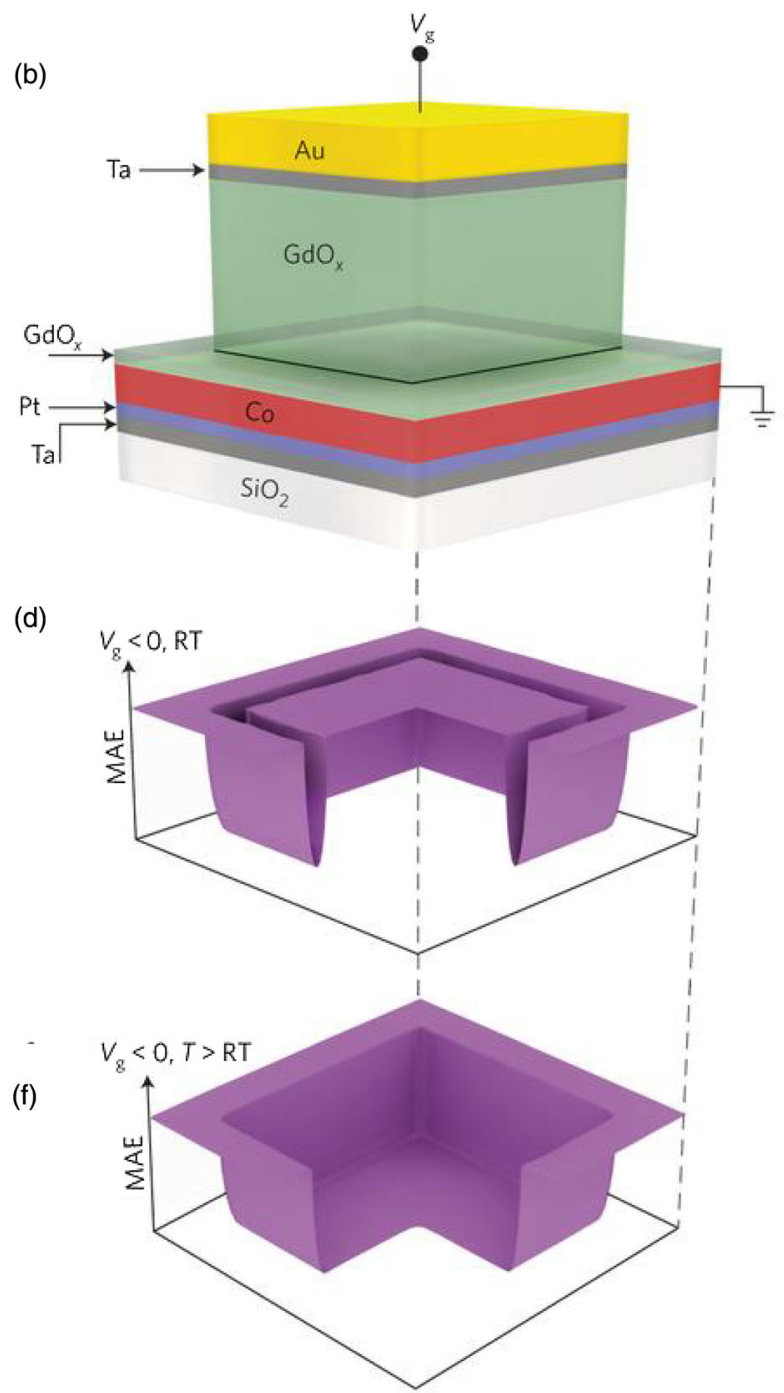

(g)

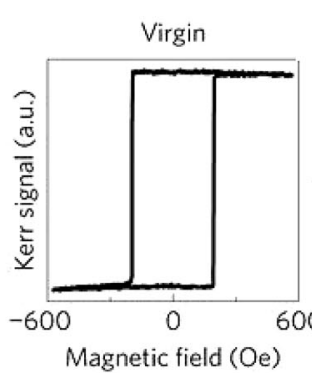

(h)

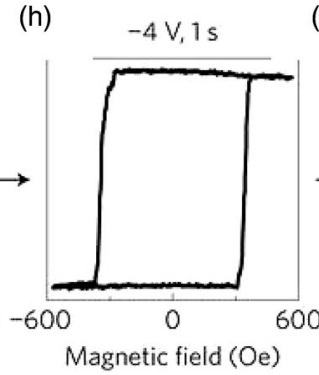

(i)
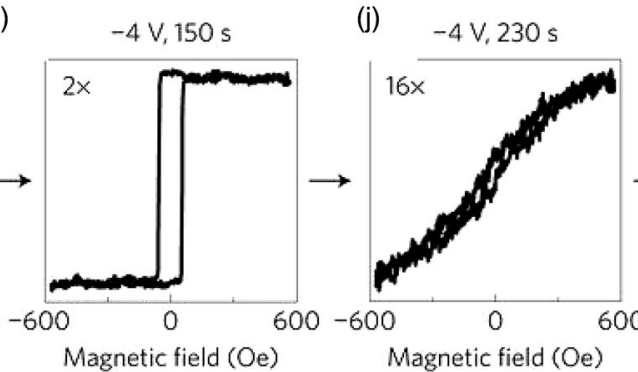

(k)

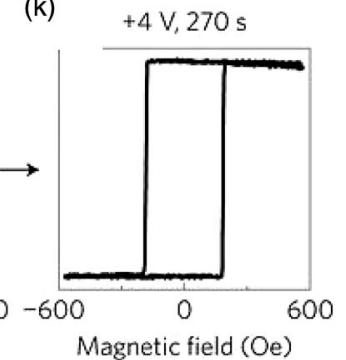

FIG. 43. Device schematics and voltage control of magnetic anisotropy. (a), (c), (e) Topographic map of the coercivity $\left(H_{c}\right)$ measured under different applied gate voltage conditions. (b) Schematic view of the gate-electrode structure; (d), (f) MAE landscapes after applying gate voltage conditions illustrated in (c) and (e), respectively. (g)-(k) Polar MOKE hysteresis loops measured at room temperature under the same conditions. From Bauer et al., 2015. 

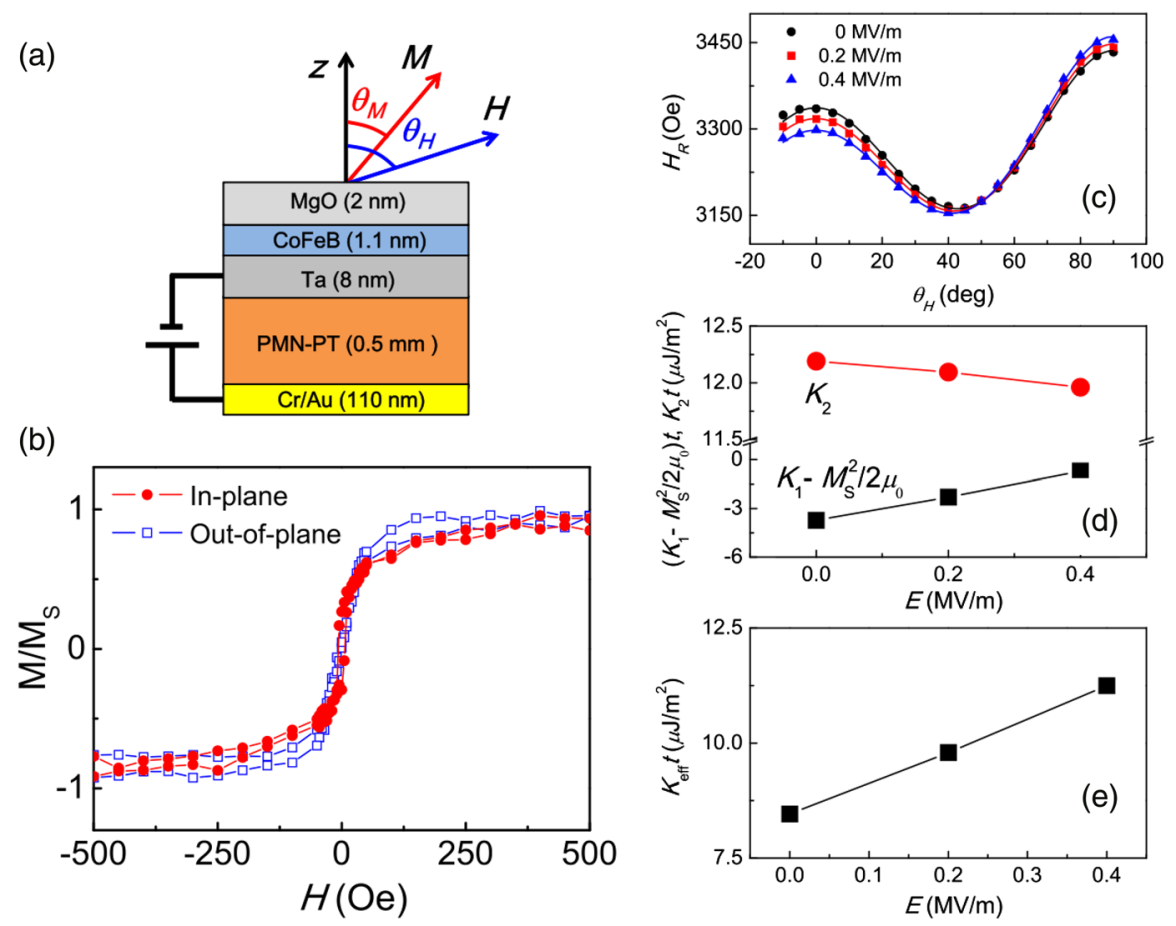

FIG. 44. (a) Schematic representation of the sample structure and the measurement configuration. (b) In-plane (red circles) and out-ofplane (blue squares) magnetic hysteresis loops of the samples at zero electric field. (c) The $\theta_{H}$ dependence of the resonance field $H_{R}$ for different electric field values $E=0,0.2$, and $0.4 \mathrm{MV} / \mathrm{m}$. (d) The electric-field dependence of first- and second-order magnetic anisotropy parameters. (e) The electric-field dependence of the effective magnetic anisotropy energy per unit area. From Yu et al., 2015.

interfaces in single, crystalline $\mathrm{V} / \mathrm{Fe} / \mathrm{MgO} / \mathrm{Fe}$ MTJ. As shown in Fig. 42, they detected no change in PMA when electrons were added at the $\mathrm{Fe} / \mathrm{MgO}$ interface. In contrast, with a positive voltage, the anisotropy constant decreased as the bias voltage increased, revealing a highly sensitive MCA with a slope value of $\sim 1150 \mathrm{fJ} /(\mathrm{V} \mathrm{m})$ and clear transition from out-of-plane to inplane magnetization orientation (Rajanikanth et al., 2013). As in Bauer et al., 2012, this large effect could be attributed to the voltage-polarity-dependent charge-trapping migration within the $\mathrm{Fe} / \mathrm{MgO}$ interfaces.

Another breakthrough experiment was recently published by Beach's group (Bauer et al., 2015). In this study, they were able to observe voltage-driven $\mathrm{O}_{2}$ migration in a $\mathrm{Co} /$ metal-oxide bilayer in situ. This migration allows electrochemical variation of interfacial MAE at unprecedentedly high amplitudes (Fig. 43). To perform this study, they prepared sputtered $\mathrm{Ta} / \mathrm{Pt} / \mathrm{Co} / \mathrm{GdOx}$ films where the interfacial MAE was varied by more than $0.75 \mathrm{erg} / \mathrm{cm}^{2}$ at just $2 \mathrm{~V}$. This value corresponds to the magnetoelectric efficiency with slope values of $>5000 \mathrm{fJ} /(\mathrm{V} \mathrm{m})$, which is much beyond the possibilities available with conventional magnetoelectric coupling mechanisms. They employed cross-sectional TEM and high-resolution electron energy loss spectroscopy to track voltage-driven migration of the oxidation front in situ in a Co/GdOx bilayer and related their observations to voltageinduced anisotropy changes. By minimizing the $\mathrm{O}_{2}$-diffusion barrier, they were able to reduce the time scale for magnetoionic switching by $\sim 6$ orders of magnitude (Bauer et al., 2015). Large PMA variation induced by oxygen migration has been explained theoretically in the case of $\mathrm{Fe} / \mathrm{MgO}$ interfaces (Ibrahim et al., 2016a).
Finally, another mechanism which also gives rise to extremely large magnetoelectric coupling efficiency for electric-field control of MCA should be discussed. This mechanism is based on voltage-induced mechanical strain in a magnetoelastic material. It turns out that voltage-induced lattice strain in a piezolelectric substrate can lead to strong modulation of the MCA in adjacent FM material. Yu et al. (2015) achieved this effect by depositing $\mathrm{Ta} / \mathrm{CoFeB} / \mathrm{MgO}$ structures on top of a piezoelectric lead

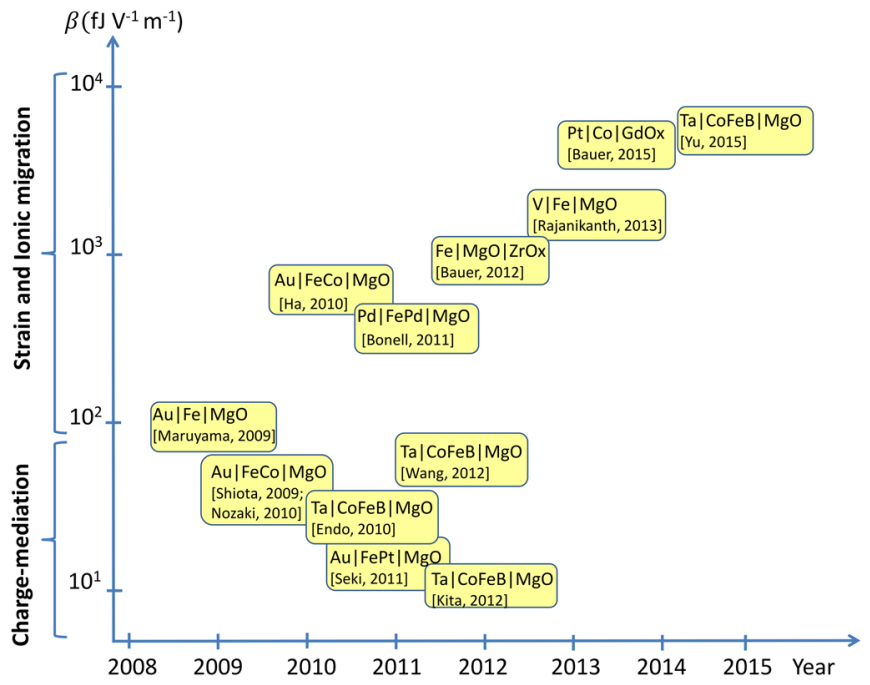

FIG. 45. A schematic summary of recent experimental reports relating to efficiency parameters of electric-field control of magnetic anisotropy at FM/oxide interfaces and in magnetic tunnel junctions. Strain and ionic migration control anisotropy more efficiently than charge-induced mechanisms. 
magnesium niobate-lead titanate substrate (PMN-PT). They produced an in-plane biaxial strain by applying voltage between the two surfaces of the PMN-PT substrate, and this strain was subsequently transferred to the ferromagnetic $\mathrm{CoFeB}$ layer, resulting in tuning of its PMA due to the magnetostriction in the CoFeB alloy. A large value for the voltage-induced PMA modulation, with a slope of $7000 \mathrm{fJ} /(\mathrm{V} \mathrm{m})$, was obtained (Yu et al., 2015). Interestingly, their results also showed that both first- and second-order anisotropy terms are affected by the electric field, and that they have opposing voltage dependencies, as shown in Fig. 44 (Yu et al., 2015).

To conclude this section, Fig. 45 schematically summarizes recent developments in electric-field control of magnetic crystalline anisotropy at transition metal/oxide interfaces. This representation shows a clear trend toward electrical switching based on highly efficient strain or ionic migration mechanisms. These mechanisms pave the way toward novel low-power applications based on magnetic metal/oxide interfaces. However, issues related to the characteristic response time, reliability, and endurance associated with these phenomena still need to be investigated.

\section{OTHER EMERGING PHENOMENA IN STRUCTURES BASED ON MAGNETIC METAL/OXIDE INTERFACIAL PMA}

In this section, we give a brief overview of several phenomena in the emerging field of spin orbitronics which greatly benefit from the use of layered structures with PMA at FM/MOx interfaces. Because these structures, typically with the form NM/FM/Ox (see, for example, Fig. 45), by definition possess structural inversion symmetry, net effects on spin and orbital moments will be induced within the structure due to SOIs. The first phenomenon to benefit from these structures is SOT arising from the Rashba effect (Manchon and Zhang, 2008; Miron et al., 2010; Miron, Garello et al., 2011; Pi et al., 2010; Suzuki et al., 2011; Pesin and MacDonald, 2012; Wang and Manchon, 2012; Garello et al., 2013; Kim et al., 2013; Yu et al., 2014) or from the spin Hall effect (Miron, Garello et al., 2011; Garello et al., 2013; Liu, Lee et al., 2012; Liu, Pai et al., 2012; Kim et al., 2013; Vedyaev et al., 2011). SOT allows highly effective switching of the magnetization direction in a thin FM layer through the application of an in-plane electric current (Miron, Garello et al., 2011; Liu, Lee et al., 2012; Yu et al., 2014). This capacity is of much technological interest for the control of magnetic memory devices and has allowed the development of novel SOT-MRAM memory concepts based on out-ofplane magnetized p-MTJs (Gaudin et al., 2010; Cubukcu et al., 2014) as well as in-plane magnetized MTJs (Liu, Pai et al., 2012; Pai et al., 2012; Yamanouchi et al., 2013). Furthermore, SOT revitalized the topic of current-induced magnetization dynamics of DW (Miron et al., 2009, 2011; Emori, Bono, and Beach, 2012) which is promising for the development of devices exploiting DW motion, such as racetrack memories (Parkin, Hayashi, and Thomas, 2008). Another spin-orbit phenomenon which is naturally present in these structures with broken inversion symmetry is the Dzyaloshinskii-Moriya interaction (DMI) (Dzialoshinskii, 1957; Moriya, 1960). This interaction stabilizes homochiral Néel-type DWs which is predicted to be crucial for their efficient motion due to the effects of SHE-induced SOT

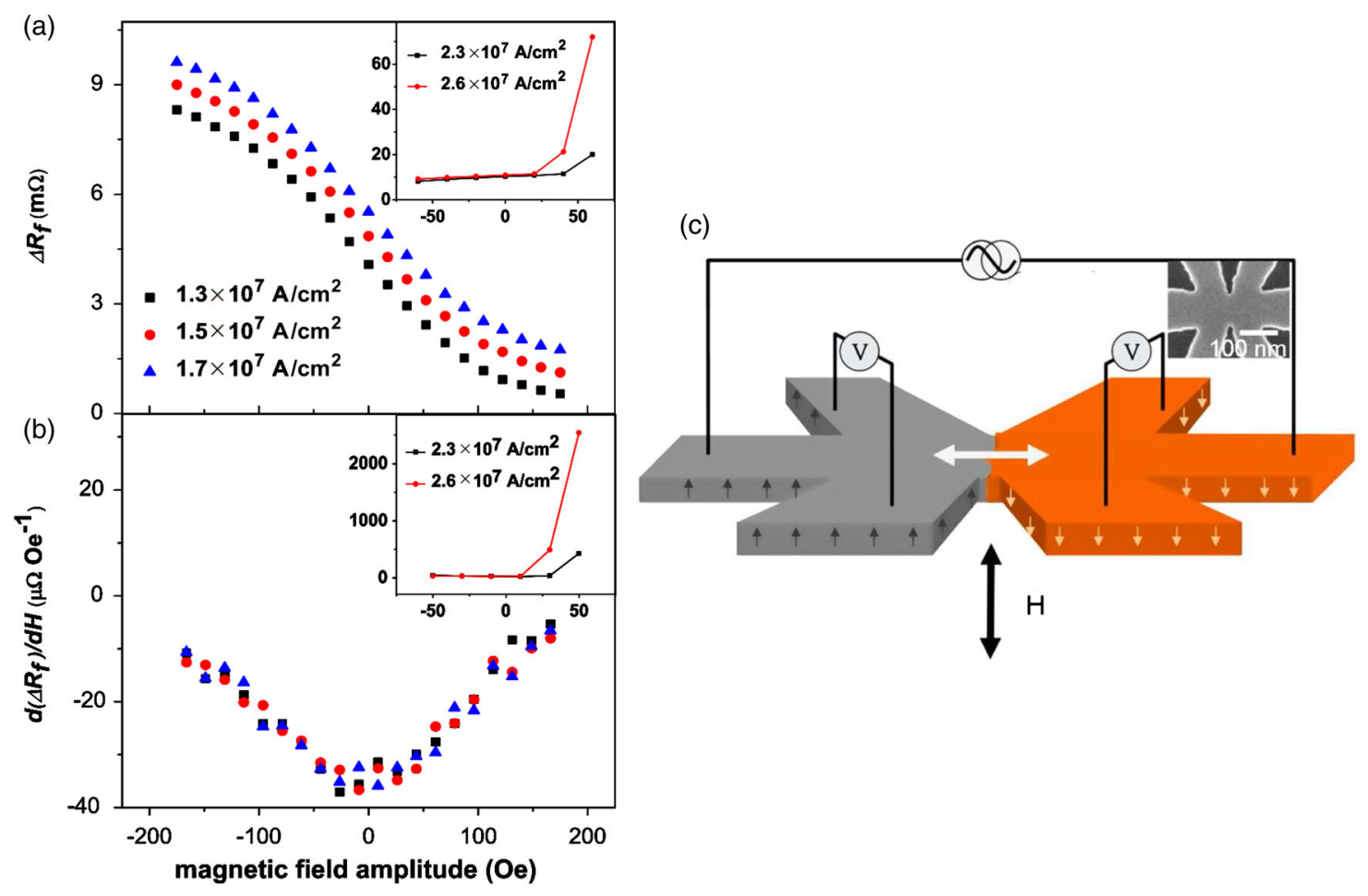

FIG. 46. The nonlinear response of a DW to a magnetic field. (a) $\Delta R_{f}$ and (b) the derivative of $\Delta R_{f}$ vs the amplitude of the magnetic field applied. Three different current densities are shown for $\mathrm{Pt} / \mathrm{Co} / \mathrm{Pt}$ layers and $\mathrm{Pt} / \mathrm{Co} / \mathrm{AlOx}$ (inset) structures. (c) Schematic representation of the experimental setup. From Miron et al., 2009. 
(a)

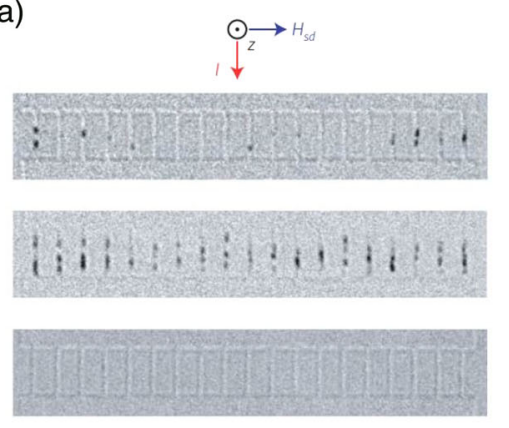

(d)

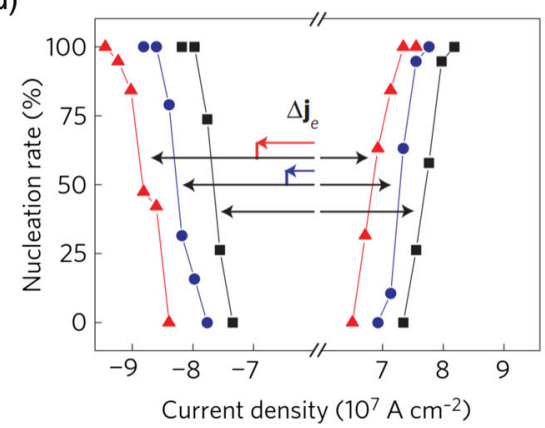

(b)

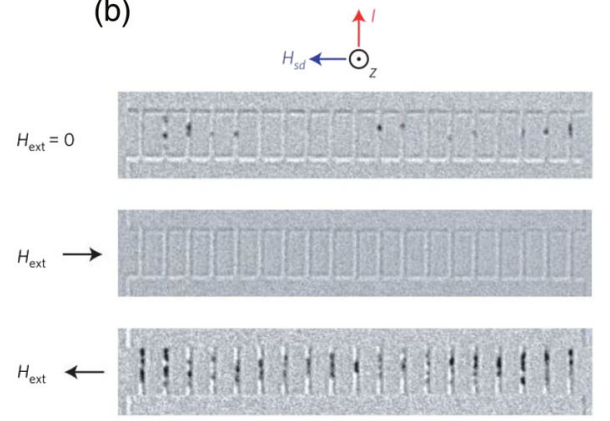

(e)

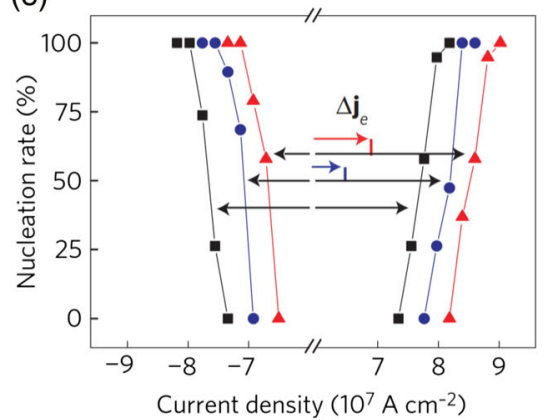

(c)

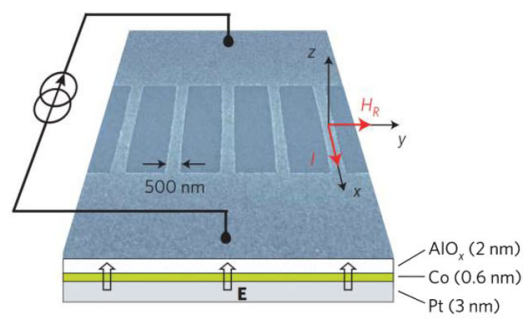

(f)

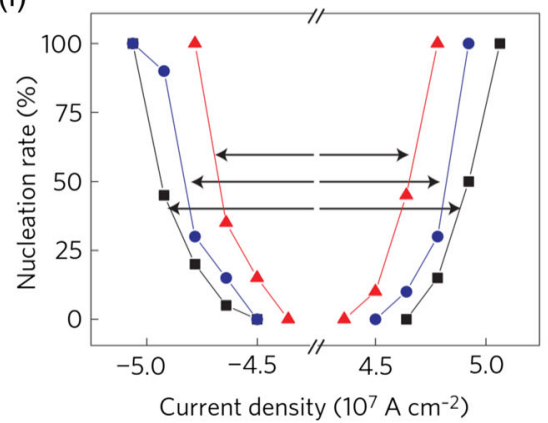

FIG. 47. (a), (b) Differential Kerr microscopy images recorded after (a) positive and (b) negative current pulse injection for applied fields with different current densities. (c) Scanning electron micrograph detail of the patterned $\mathrm{Pt} / \mathrm{Co} / \mathrm{AlOx}$ wire array and schematic representation of the vertical cross section of the layer. Arrows indicate the direction of current flow, interfacial electric field, and corresponding Rashba field. (d)-(f) Proportion of wires that presents reverse magnetic domains after the injection of a current pulse as a function of current density and external field. The nucleation rate curves shift by an amount $\Delta j_{e}$ proportional to $H_{\text {ext }}$, as indicated by the red and blue arrows, reflecting the direction and magnitude of the Rashba field for (a), (b) $\mathrm{Pt} / \mathrm{Co} / \mathrm{AlOx}$ wire arrays to which (a) positive and (b) negative fields were applied; (c) $\mathrm{Pt} / \mathrm{Co} / \mathrm{Pt}$ wire array. Blue dots, black squares, and red triangles corresponds to applied external field cases of $0, \pm 47.5$, and $\pm 95 \mathrm{mT}$, respectively. (g) Relationship between current density and magnetic field for Pt/Co/AlOx and $\mathrm{Pt} / \mathrm{Co} / \mathrm{Pt}$ samples. From Miron et al., 2010.

(a)

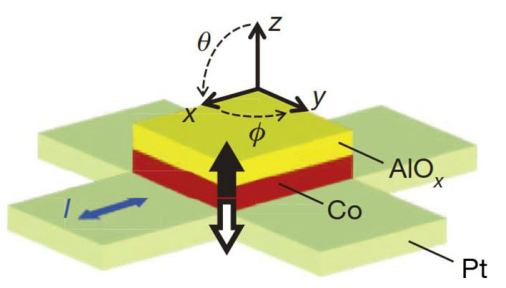

(b)

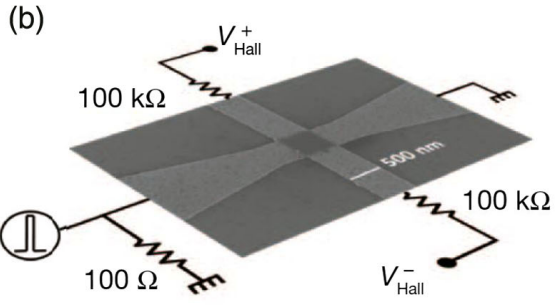

(c)

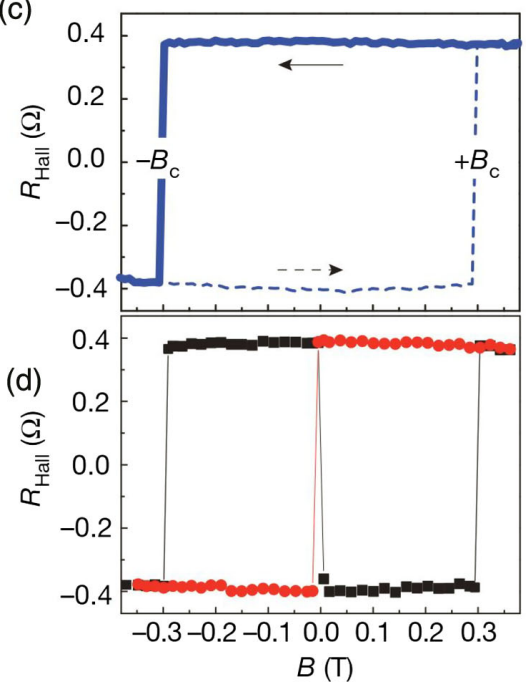

(e)
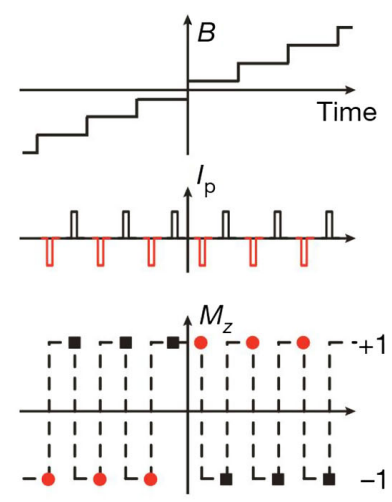

FIG. 48. (a) Hall cross geometry and (b) scanning electron micrograph of the sample and electric circuitry used to observe SOTinduced switching in $\mathrm{Pt} / \mathrm{Co} / \mathrm{AlOx}$ out-of-plane magnetized structures. Black and white arrows indicate the "up" and "down" equilibrium magnetization states of the cobalt layer, respectively. (c), (d) Longitudinal magnetization component measured based on the anomalous Hall resistance as a function of applied field after the injection of positive (black squares) and negative (red circles) current pulses. (e) Schematic representation of the pulse sequence and magnetization measurements. From Miron, Garello et al., 2011. 
(Thiaville et al., 2012). This requirement was confirmed experimentally in structures with PMA including $\mathrm{Pt} / \mathrm{CoFe} / \mathrm{MgO}$ and $\mathrm{Ta} / \mathrm{CoFe} / \mathrm{MgO}$ (Emori et al., 2013). It also leads to the dynamic tilting of DW driven by SOT (Boulle et al., 2013). Moreover, the combination of PMA and DMI in thin films makes them an ideal system for the nucleation and propagation of topological textures known as Skyrmions (Bogdanov and Röszligler, 2001; Roessler, Bogdanov, and Pfleiderer, 2006; Mühlbauer et al., 2009; Yu et al., 2010; Heinze et al., 2011), opening a way for ultradense information storage systems (Fert, Cros, and Sampaio, 2013; Iwasaki, Mochizuki, and Nagaosa, 2013; Sampaio et al., 2013). Recently, Jiang et al. (2015) demonstrated that SOTs can effectively generate mobile magnetic Skyrmions at RT in out-of-plane magnetized $\mathrm{Ta} / \mathrm{CoFeB} / \mathrm{TaOx}$ structures. RT magnetic Skyrmions have also been observed in $\mathrm{Pt} / \mathrm{Co} / \mathrm{MgO}$ structures with PMA (Boulle et al., 2016). Overall, all these examples indicate that layered structures with structural inversion asymmetry including FM/MOx interfaces with PMA have opened up avenues toward novel paradigms in spintronic devices, particularly in the emerging field of spin orbitronics. Next we briefly summarize several studies which are considered milestones in this area. We have concentrated on those employing FM metal/MOx interfaces and MTJs with PMA. Recent findings indicate that SOT and other spin-orbitronic phenomena do not require the presence of a heavy metal, and that these phenomena may be observed in structures with weak spin-orbit metals (Du et al., 2014; Emori et al., 2016) or even structures composed only of FM/MOx interfaces (Emori et al., 2016; Mouillon et al., 2016). Furthermore, Emori et al. (2016) recently reported that SOT can arise solely due to interfacial spin-orbit-coupling effects at metal/insulator interfaces (Rashba mechanism); similar findings were reported by Mouillon et al. (2016). This effect can be associated with the formation of an interfacial dipole at transition metal/oxide interfaces (Ibrahim et al., 2016b). In addition to the aforementioned correlation between the PMA and intrinsic electric dipolar field, it has been shown that the corresponding Rashba magnetic field at the interface may affect the PMA (Barnes, Ieda, and Maekawa, 2014).

The purpose of the first study performed by Miron et al. (2009) was to directly measure the nonadiabatic component of the spin torque in DW. This required very high spin flip rates (Zhang and Li, 2004) which can be achieved in systems exhibiting a strong crystalline field within the FM layer along with a breaking of the inversion symmetry, since otherwise the total torque of the magnetic field on the electron spin averages out, and the spin flip would only be possible during momentum scattering (Fabian and Das Sarma, 1999). Out-of-plane magnetized $\mathrm{Pt} / \mathrm{Co} / \mathrm{AlOx}$ and $\mathrm{Pt} / \mathrm{Co} / \mathrm{Pt}$ samples with double EHE crosses were patterned and magnetically prepared with a domain wall trapped between the two crosses (Fig. 46). The deformation of the wall due to application of an out-of-plane field and an in-plane current could then be monitored in the lowfrequency ac mode [Fig. 46(c)]. The current-induced DW motion was tracked by monitoring variations in resistance which produced a nonlinear relationship between the voltage measured and the current applied (Miron et al., 2009). For asymmetric $\mathrm{Pt} / \mathrm{Co} / \mathrm{AlOx}$ structures, this relationship was found to be highly asymmetric and to depend on the current amplitude nonlinearities in $\Delta R_{f}$ as well as its derivative when plotted against field amplitude. In contrast, a fully symmetric (a)

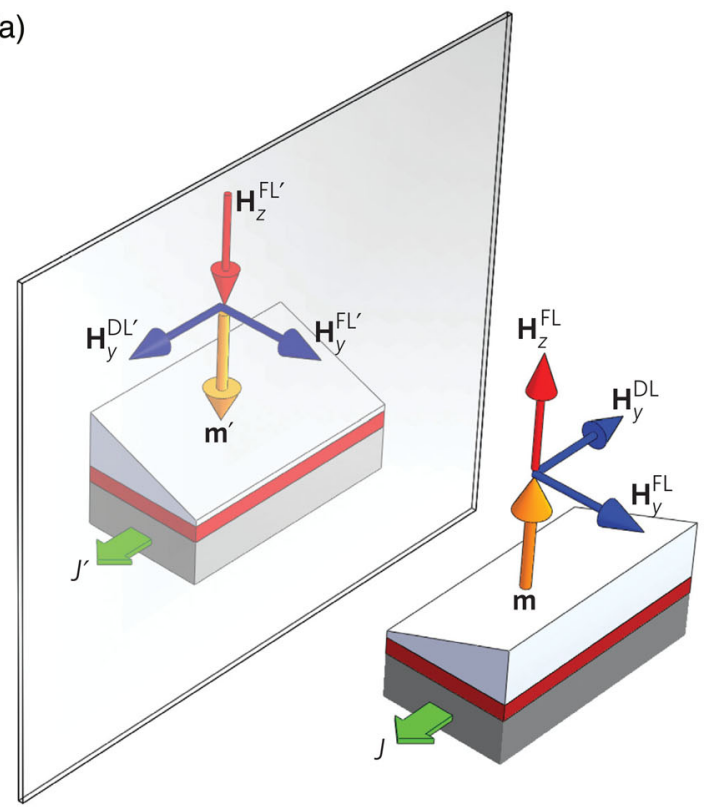

(b)

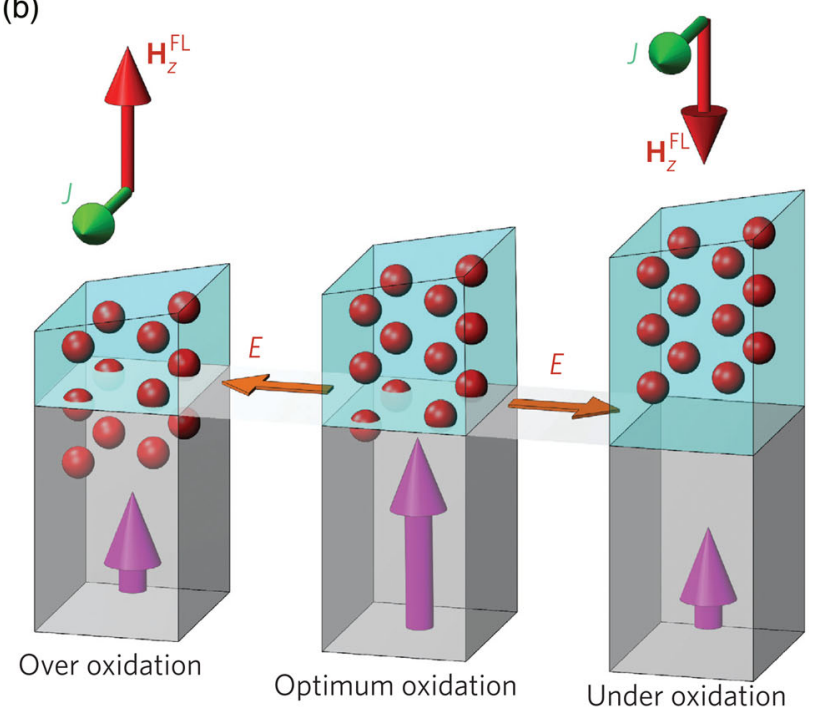

FIG. 49. (a) The new perpendicular effective field induced by the laterally asymmetric structure and its mirror image. The presence of the new perpendicular effective field, induced by the lateral symmetry breaking, uniquely determines the $z$ component of the magnetization for a particular direction of current, thereby allowing deterministic switching without the need to apply external magnetic fields. (b) Schematic representation of the ferromagnet/oxide interface, illustrating its nonuniform oxygen content. The resulting nonuniform charge distribution may produce in-plane electric fields along the interface, which in turn can contribute to the out-of plane field component. Red spheres indicate oxygen atoms and perpendicular pink arrows correspond to the PMA in the magnetic layer. From Yu et al., 2014. 
(a)

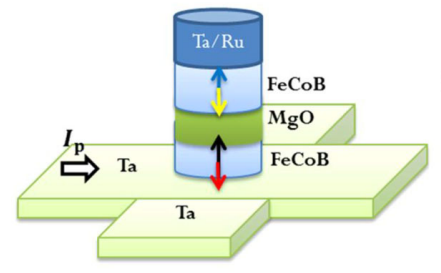

(c)

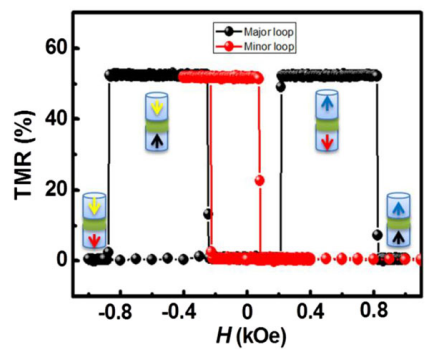

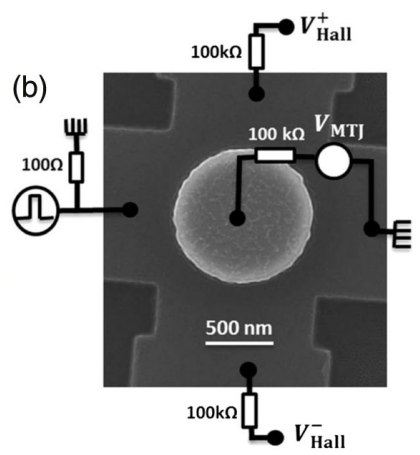

(d)

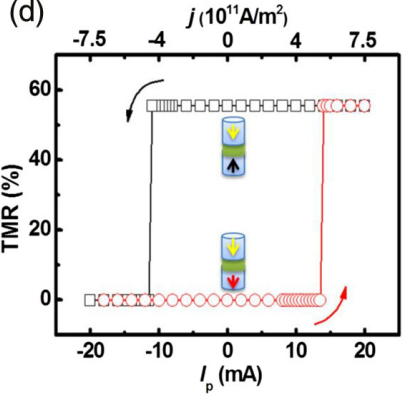

FIG. 50. (a) Illustration of a three-terminal MTJ with a Hall cross geometry. The black (blue) "up" and red (yellow) "down" arrows indicate the equilibrium magnetization states of the bottom (top) FeCoB layers. (b) Scanning electron microscopy image of a 1- $\mu \mathrm{m}-$ diameter MTJ dot on top of a 1.3- $\mu \mathrm{m}$-wide Ta electrode with a schematic representation of the electrical measurement setup. (c) TMR as a function of the perpendicular magnetic field for a $1-\mu \mathrm{m}$ dot diameter. The black and red curves show a major and a minor loop, respectively. (d) TMR as a function of current pulse amplitude injected into the Ta electrode using 50-ns pulses under an in-plane magnetic field $(-0.4 \mathrm{kOe})$. The arrows show the sweep direction for the current pulse. From Cubukcu et al., 2014.

dependence, which was independent of the current amplitude, was measured for symmetric $\mathrm{Pt} / \mathrm{Co} / \mathrm{Pt}$ samples [Figs. 46(a) and 46(b) and insets]. Based on these results, the nonadiabatic spintorque component was found to be 2 orders of magnitude higher within $\mathrm{Pt} / \mathrm{Co} / \mathrm{AlOx}$ samples than in $\mathrm{Pt} / \mathrm{Co} / \mathrm{Pt}$ ones. They explained this difference using the fact that spin-orbit coupling is much stronger in the case of asymmetric structures due to the Rashba interaction (Miron et al., 2009), and this was the first documented manifestation of SOT on DW dynamics. It should be pointed out here that the symmetric sputtered $\mathrm{Pt} / \mathrm{Co} / \mathrm{Pt}$ samples used in these experiments were not ideally symmetric. Their nonideal symmetry was revealed, for instance, by DMI measurements performed on these structures (Je et al., 2013; Hrabec et al., 2014) since otherwise the DMI contributions from two interfaces would cancel out (Yang et al., 2015).

This suspected SOT was subsequently clearly evidenced by the same group in $\mathrm{Pt} / \mathrm{Co} / \mathrm{AlOx}$ wires by systematic investigation of magnetic domain nucleation rates under positive and negative currents and fields (Miron et al., 2010). As seen in Fig. 47, strong amplification or suppression of domain nucleation rates was observed depending on the sign of both current and external field inducing the shift in current of the nucleation curves. This shift is therefore directly proportional to the applied field in line with theoretical predictions of the Rashba-induced fields and spin-orbit torques (Manchon and

(a)

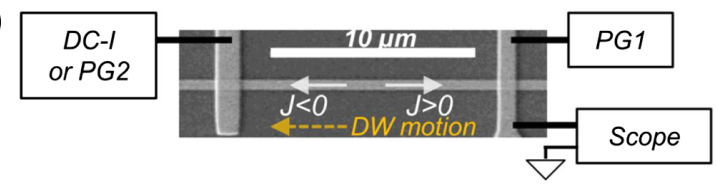

(b)

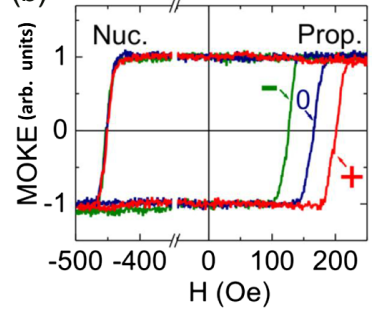

(c)

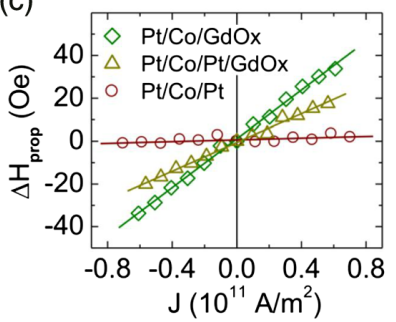

FIG. 51. (a) Micrograph of a Pt/Co/GdOx strip and schematic representation of the measurements performed. With $J<0$, the current is in the same direction as (and electron flow opposes) field-driven DW motion. (b) Hysteresis loops showing that the DW propagation field changes and the nucleation field remains invariant with variations in injected dc current density $\left(J=-0.61,0\right.$, and $\left.+0.61 \times 10^{11} \mathrm{~A} / \mathrm{m}^{2}\right)$. (c) DW propagation field change $\Delta H_{\text {prop }}$ as a function of the dc current density injected into $\mathrm{Pt} / \mathrm{Co} / \mathrm{GdOx}, \mathrm{Pt} / \mathrm{Co} / \mathrm{Pt} / \mathrm{GdOx}$, and $\mathrm{Pt} / \mathrm{Co} / \mathrm{Pt}$ strips. From Emori, Bono, and Beach, 2012.

Zhang, 2008). This was one of the first experimental observations of the very large SOT induced by an electric current flowing through a uniformly magnetized ferromagnetic metal layer originating from structural inversion asymmetry (Miron et al., 2010; Gambardella and Miron, 2011).

The next significant step forward was the demonstration that switching could be induced in a perpendicularly magnetized cobalt dot within a $\mathrm{Pt} / \mathrm{Co} / \mathrm{AlOx}$ structure by the application of in-plane current injection at RT (Miron, Garello et al., 2011). Magnetization switching from up to down and vice versa was observed following every pulse of current, with this switching being controlled by the sign of the applied field (Fig. 48). This behavior was found to be independent of the sample's domain configuration. It is remarkably different from that expected for known magnetic interactions (Miron, Garello et al., 2011). The switching efficiency was found to increase with the MA of the cobalt layer and the oxidation of the aluminum layer, suggesting that the structural inversion asymmetry effects, including Rashba and spin Hall effects, played a key role in the reversal mechanism. Finally, to prove that in-plane current switching has the potential for spintronic applications, they also developed a reprogrammable magnetic switch that can be integrated into nonvolatile memory and logic architectures providing a simple, scalable device which is compatible with present-day magnetic recording technology (Miron, Garello et al., 2011). These results were later reproduced for $\mathrm{Pt} / \mathrm{Co} / \mathrm{AlOx}, \mathrm{Pt} / \mathrm{Co} / \mathrm{MgO}$, and $\mathrm{Ta} / \mathrm{CoFeB} / \mathrm{MgO}$ structures which display PMA (Avci et al., 2012; Liu, Lee et al., 2012). However, Liu, Lee et al. strongly emphasized that the driving SOT mechanism originates from a spin Hall effect, which triggered a discussion on the relative importance of Rashba versus spin Hall effects (Wang and Manchon, 2012; Garello et al., 2013; Kim et al., 2013). An important study shedding light on this discussion was published by Kim et al. (2013). They systematically performed vectorial measurements of the current-induced effective field associated 
(a)

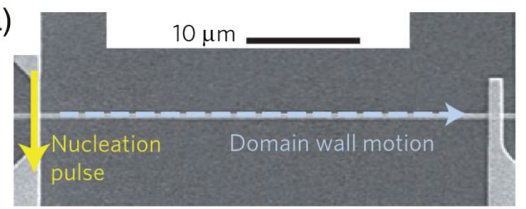

(b)

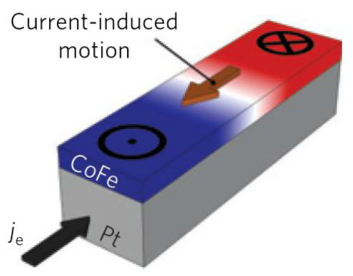

(c)

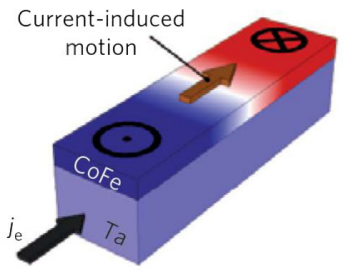

(d)

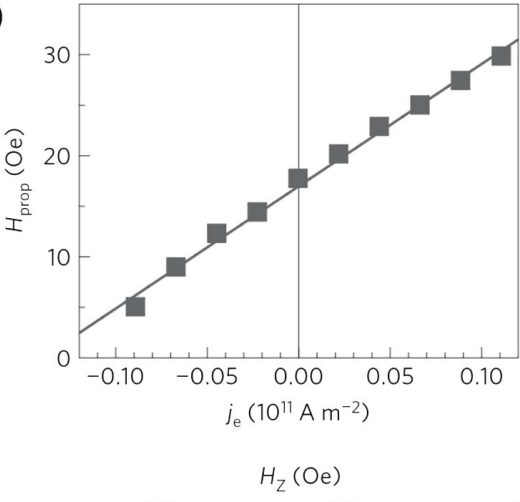

(f)

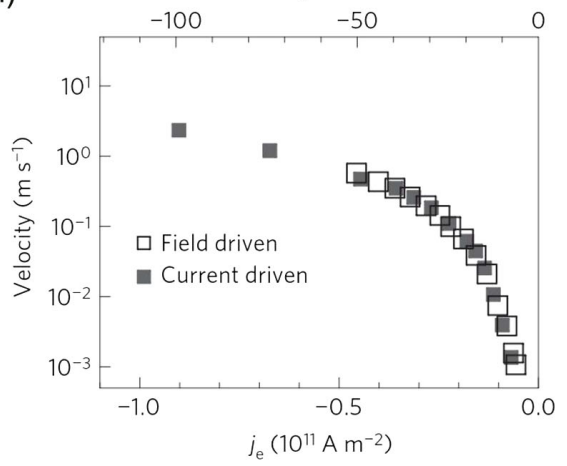

(e)

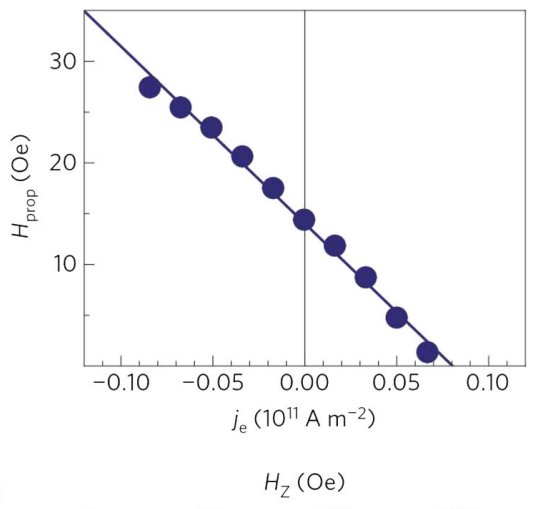

(g)

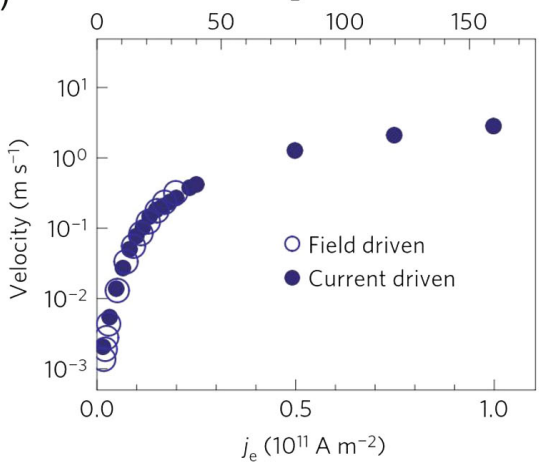

FIG. 52. (a) Scanning electron micrograph of the nanowire. The current pulse on the left nucleates a DW, which is then propagated to the right by applying current or an out-of-plane field. Illustrations of the direction of current-driven DW motion in the (b) $\mathrm{Pt} / \mathrm{CoFe} / \mathrm{MgO}$ and (c) $\mathrm{Ta} / \mathrm{CoFe} / \mathrm{MgO}$ nanowires. Electron current is defined as positive when conduction electrons flow away from the nucleation line, i.e., from left to right in the micrograph (a). DW propagation field $H_{\text {prop }}$ vs driving electron current density for (d) $\mathrm{Pt} / \mathrm{CoFe} / \mathrm{MgO}$ and (e) $\mathrm{Ta} / \mathrm{CoFe} / \mathrm{MgO}$. Te slope of the linear fit gives the spin-torque efficiency for each structure. DW velocity as a function of $j$ and applied out-of-plane field $H_{z}$ for (f) $\mathrm{Pt} / \mathrm{CoFe} / \mathrm{MgO}$ and (g) $\mathrm{Ta} / \mathrm{CoFe} / \mathrm{MgO}$. From Emori et al., 2013.

with SOT in $\mathrm{Ta} / \mathrm{CoFeB} / \mathrm{MgO}$ and quantitatively determined their size and direction. They found that both the size and the sign of the transverse and longitudinal effective fields varied in proportion to the thickness of the Ta layer. This apparent relationship suggested competing contributions from two distinct sources arising from Rashba and spin Hall mechanisms (Kim et al., 2013). The symmetry properties of SOT were further clarified by Garello et al. (2013), who reported threedimensional vectorial measurements of SOTs in $\mathrm{Pt} / \mathrm{Co} / \mathrm{AlOx}$ and $\mathrm{Ta} / \mathrm{CoFeB} / \mathrm{MgO}$ trilayers by applying harmonic analysis to the anomalous and planar Hall effects.

A general scheme to measure the amplitude and direction of SOTs as a function of the magnetization direction was provided. Based on symmetry arguments related to space and time inversion, they demonstrated that heavy metal and ferromagnetic layers can be associated with two different SOTs which display odd and even behavior with respect to magnetization reversal. These torques include strongly anisotropic fieldlike and spin-transfer-like components, which depend on the type of heavy metal layer and annealing treatment as well as showing nontrivial angular dependencies (Garello et al., 2013). These dependences were also supported theoretically (Ortiz Pauyac et al., 2013).

An interesting SOT switching mechanism which does not require external magnetic fields has been proposed to exist in $\mathrm{Ta} / \mathrm{CoFeB} / \mathrm{TaOx}$ devices by $\mathrm{Yu}$ et al. (2014). They introduced a lateral structural asymmetry which plays the role of the external in-plane magnetic field giving rise to a new out-of-plane fieldlike SOT when in-plane current flows through these structures. This SOT facilitates the switching of the magnetization direction in perpendicular magnets [Fig. 49(a)]. Interestingly, the lateral asymmetry was induced by nonuniform oxidation of the $\mathrm{TaOx}$ layer. This produces an in-plane electric field which in turn gives rise to the out-ofplane effective field component [Fig. 49(b)].

The potential to use current-induced SOT switching opened a route toward SOT-MRAM configurations. Preliminary designs were based on a three-terminal spin Hall switching device comprising in-plane magnetized $\mathrm{CoFeB} / \mathrm{MgO} / \mathrm{CoFeB}$ MTJs (Liu, Pai et al., 2012; Yamanouchi et al., 2013). The first threeterminal device based on MTJs with PMA was realized by Cubukcu et al. (2014). A perpendicular SOT-MRAM cell is composed of a $\mathrm{FeCoB} / \mathrm{MgO} / \mathrm{FeCoB}$ MTJ deposited on top of a 20-nm-thick Ta current line (Fig. 50). The abrupt MTJ resistance change observed during the field sweep shows that magnetization of the two magnetic layers is perpendicular. Simultaneous measurement of the EHE shows that the top electrode switches first when sweeping the magnetic field [Fig. 50(c)]. Starting from a parallel configuration, the in-plane injection of a large enough positive current pulse leads to an increase of the MTJ resistance, revealing the reversal of the magnetization of the bottom $\mathrm{FeCoB}$ layer. From the antiparallel configuration, the magnetization can be switched back to a parallel configuration by injecting a negative in-plane current pulse. The bottom $\mathrm{FeCoB}$ magnetization can thus be switched hysteretically by current pulse injection in the bottom Ta 
electrode in the presence of an in-plane field and its orientation detected by the TMR signal [Fig. 50(d)] (Cubukcu et al., 2014).

The presence of the SOT in this system also turned out to be efficient for fast DW motion in $\mathrm{Pt} / \mathrm{Co} / \mathrm{AlOx}$ nanowires, as reported by Miron et al. (2011). They found that subnanosecond current pulses induced reproducible DW displacements at high velocities, which was an essential step toward the development of viable devices. Interestingly, a field current equivalence was found between this DW motion and the quasistatic measurements performed at lower current densities mentioned previously (Miron et al., 2009) (far below the Walker breakdown) on the same system up to the highest current densities. This proves that the same physical mechanism governs the domain wall motion in these different regimes (Miron et al., 2011).

Current-induced DW propagation induced by large SOT was also reported by Emori, Bono, and Beach (2012) for $\mathrm{Pt} / \mathrm{Co} / \mathrm{GdOx}$ nanostrips when investigating MOKE hysteresis loops under positive and negative currents to extract the DW propagation fields [Figs. 51(a) and 51(b)]. A strong decrease in the propagation-field-to-injected-current-density ratio was observed when a thin Pt layer was inserted between the Co film and the GdOx overlayer [Fig. 51(c)]. Moreover, the currentinduced effects completely vanished in symmetric Pt $3 \mathrm{~nm} / \mathrm{Co}$ $0.9 \mathrm{~nm} / \mathrm{Pt} 3 \mathrm{~nm}$ strips, suggesting that the $\mathrm{Co} / \mathrm{GdOx}$ interface plays a direct role in generating the large current-induced torque observed (Emori, Bono, and Beach, 2012). This hypothesis is in agreement with earlier reports for $\mathrm{Pt} / \mathrm{Co} / \mathrm{AlOx}$ systems (Miron et al., 2009, 2010, 2011). However, it was pointed out that no clear evidence of a strong effective Rashba transverse field had been presented (Emori, Bono, and Beach, 2012). Indeed, the discussion on the relative importance of spin Hall versus Rashba mechanisms has a direct impact on current-induced DW motion under the influence of SOT, especially in structures with FM/MOx PMA.

Emori et al. (2013) found that SOT due to the spin Hall effect drives DWs in opposite directions in perpendicularly magnetized $\mathrm{Pt} / \mathrm{CoFe} / \mathrm{MgO}$ and $\mathrm{Ta} / \mathrm{CoFe} / \mathrm{MgO}$ structures. This effect can be explained only if the DWs assume a homochiral Néel configuration which is produced and stabilized by the DMI (Thiaville et al., 2012). DW motion was characterized in nanowires overlaid with an orthogonal DW nucleation line and lateral contacts for current injection [Fig. 52(a)]. The propagation field was found to vary in direct proportion to the electron current density, but DW propagation was hindered in the electron flow direction in $\mathrm{Pt} / \mathrm{CoFe} / \mathrm{MgO}$ and assisted in the same direction as electron flow in $\mathrm{Ta} / \mathrm{CoFe} / \mathrm{MgO}$ [Figs. 52(d) and 52(e)]. This remarkable difference, produced simply by changing the NM in contact with the ferromagnet, was found to be independent of the magnetization direction (up down or down up) across the DW, suggesting that a universal mechanism governs currentdriven DW motion in heavy metal/ferromagnet/oxide systems (Emori et al., 2013). A comparison of field-driven and currentdriven DW velocities showed that DWs moved against electron flow in $\mathrm{Pt} / \mathrm{CoFe} / \mathrm{MgO}$ [Fig. 52(f)] and along electron flow in $\mathrm{Ta} / \mathrm{CoFe} / \mathrm{MgO}$ [Fig. 52(g)]. The field-to-current ratios closely matched those extracted from Figs. 52(d) and 52(e). Therefore they concluded that the effect of the current on DW motion is phenomenologically equivalent to an out-of-plane field (Miron et al., 2009; Emori, Bono, and Beach, 2012) and that the main mechanism driving the DW in opposite directions is the spin Hall effect, provided that a strong DMI is also present (Emori et al., 2013).

\section{CONCLUSION}

The phenomenon of perpendicular anisotropy at magnetic metal/oxide interfaces was first discovered by Monso et al. (2002) and extensively studied at SPINTEC since then (Rodmacq et al., 2003, 2009; Manchon et al., 2008; Dahmane et al., 2009; Nistor et al., 2009, 2010; Yang et al., 2011; Hallal et al., 2013). This phenomenon became the subject of considerable attention after 2010, following publication of a report by Ikeda et al. (2010) showing that interfacial PMA at $\mathrm{CoFeB} / \mathrm{MgO}$ interfaces could be used in STT-MRAM cells exhibiting large TMR and low STT switching current. The phenomenon is now well understood from a theoretical point of view thanks to several $a b$ initio studies which sought to understand the mechanisms involved; good agreement with experimental data was found. Several new research areas have recently emerged which exploit the PMA at magnetic metal/oxide interfaces. One of these areas is the voltage control of anisotropy which can be greatly enhanced by charge trapping or ionic migration phenomena. Others are related to inversion-symmetry-breaking effects in sandwiches composed of a heavy metal underlayer, a ferromagnetic layer, and an oxide layer. Among these research areas, SOT phenomena (in particular, Rashba and spin Hall effects), domain wall propagation, and Skyrmion formation in the presence of DMI interactions are receiving particular attention as part of the emerging field of spin orbitronics. All these topics are of great interest from both basic research and applied research vantage points. Note that the PMA at magnetic metal/oxide interfaces is so sensitive to the degree of oxidation of the interface (the presence of oxygen vacancies, etc.) that it can also be used to characterize the quality of the tunnel barrier interface, a property which was already suggested in 2002 (Monso et al., 2002). It is also interesting to note that the magnetic phenomena associated with ionic migration close to magnetic metal/oxide interfaces such as the voltage control of anisotropy establishes a connection between these studies and the intense ongoing investigations related to resistive RAM memories which themselves rely on the migration of ions or vacancies in some specific oxide layers or bilayers to provoke resistance changes [for a review, see, for instance, Akinaga and Shima (2010)]. Finally, the PMA at the interfaces between transition metals and other nonmetallic materials such as graphene has started to attract significant attention, in particular, in the emerging field of graphene spintronics, for example, through the development of Co/graphene interfaces (Vo-Van et al., 2010; Yang et al., 2016).

\section{ACRONYMS}

$\begin{array}{ll}\text { BER } & \text { bit error rate } \\ \text { CEMS } & \text { conversion electron Mössbauer spectros- } \\ & \text { copy }\end{array}$




\begin{tabular}{|c|c|}
\hline $\mathrm{cc}$ & body centered cubic \\
\hline CIP & current in plane \\
\hline DMI & Dzyaloshinskii-Moriya interaction \\
\hline $\mathrm{OS}$ & density of states \\
\hline DRAM & dynamic random access memory \\
\hline DW & domain wall \\
\hline EHE & extraordinary Hall effect \\
\hline$c$ & face centered cubic \\
\hline FM & ferromagnet \\
\hline FMR & ferromagnetic resonance \\
\hline GMR & giant magnetoresistance \\
\hline HAMR & heat assisted magnetic recording \\
\hline HDD & hard disk drives \\
\hline IDM & integrated devices manufacturer \\
\hline IMA & in-plane magnetic anisotropy \\
\hline iPMA & $\begin{array}{l}\text { interfacial perpendicular magnetic aniso- } \\
\text { tropy }\end{array}$ \\
\hline ITRS & $\begin{array}{l}\text { international technology roadmap for } \\
\text { semiconductors }\end{array}$ \\
\hline MA & magnetic anisotropy \\
\hline MAE & magnetic anisotropy energy \\
\hline MAMR & microwave-assisted magnetic recording \\
\hline MCA & magnetocrystalline anisotropy \\
\hline ML & monolayer \\
\hline MOx & metal oxide \\
\hline MTJ & magnetic tunnel junction \\
\hline MOKE & magneto-optic Kerr effect \\
\hline MRAM & magnetic random access memory \\
\hline NM & nonmagnetic \\
\hline PMA & perpendicular magnetic anisotropy \\
\hline p-MTJ & $\begin{array}{l}\text { perpendicularly (out-of-plane) magnet- } \\
\text { ized MTJ }\end{array}$ \\
\hline $\mathrm{rf}$ & radio frequency \\
\hline rms & root mean square \\
\hline RT & room temperature \\
\hline SOI & spin-orbit interaction \\
\hline SOT & spin-orbit torque \\
\hline SRAM & static random access memory \\
\hline STT & spin-transfer torque \\
\hline STT-MRAM & $\begin{array}{l}\text { spin-transfer-torque magnetic random ac- } \\
\text { cess memory }\end{array}$ \\
\hline SQUID & $\begin{array}{l}\text { superconducting quantum interference } \\
\text { device }\end{array}$ \\
\hline TMR & tunnel magnetoresistance \\
\hline VCMA & voltage control of magnetic anisotropy \\
\hline XAS & x-ray absorption spectroscopy \\
\hline XMCD & $\mathrm{x}$-ray magnetic circular dichroism \\
\hline XPS & $\mathrm{x}$-ray photoemission spectroscopy \\
\hline
\end{tabular}

\section{ACKNOWLEDGMENTS}

We acknowledge our colleagues from SPINTEC who have contributed to all the experimental and theoretical work on this topic since 2002, in particular, our late colleague B. Rodmacq to whom we dedicate this review. We also thank S. Auffret, S. Bandiera, O. Boulle, L. D. Buda-Prejbeanu-Buda, L. Cuchet, C. Ducruet, K. Garello, G. Gaudin, A. Hallal, F. Ibrahim, J. H. Lee, A. Manchon, I. M. Miron, S. Monso, L.E. Nistor, I. L. Prejbeanu, R. C. Sousa, A. Timopheev, and H.X. Yang for their contributions. We are also very thankful to S. Andrieu, W. H. Butler, A. Fert, H. Jaffres, Y. Lu, O. Mryasov, and E. Tsymbal for helpful insights and fruitful discussions. The financial support of the European Union through ERC Adv Grant MAGICAL No. 669204 during the writing of this review is also acknowledged.

\section{REFERENCES}

Abate, E., and M. Adsente, 1965, "Tight-binding calculation of 3d bands of Fe with and without spin-orbit coupling," Phys. Rev. 140, A1303.

Akinaga, H., and H. Shima, 2010, "Resistive Random Access Memory (ReRAM) based on metal oxides," Proc. IEEE 98, 2237-2251.

Aliev, F. G., J. P. Cascales, A. Hallal, M. Chshiev, and S. Andrieu, 2014, "Band-edge noise spectroscopy of a magnetic tunnel junction,” Phys. Rev. Lett. 112, 216801.

Andersson, C., B. Sanyal, O. Eriksson, L. Nordström, O. Karis, D. Arvanitis, T. Konishi, E. Holub-Krappe, and J. Hunter Dunn, 2007, "Influence of Ligand States on the Relationship between Orbital Moment and Magnetocrystalline Anisotropy," Phys. Rev. Lett. 99, 177207.

Avci, C. O., K. Garello, I. M. Miron, G. Gaudin, S. Auffret, O. Boulle, and P. Gambardella, 2012, "Magnetization switching of an $\mathrm{MgO} / \mathrm{Co} / \mathrm{Pt}$ layer by in-plane current injection," Appl. Phys. Lett. 100, 212404.

Bae, J. S., K. H. Shin, T. D. Lee, and H. M. Lee, 2002, "Study of the effect of natural oxidation and thermal annealing on microstructures of $\mathrm{AlO}_{x}$ in the magnetic tunnel junction by high-resolution transmission electron microscopy," Appl. Phys. Lett. 80, 11681170 .

Bai, Z., L. Shen, G. Han, and Y. P. Feng, 2012, "Data storage: Review of Heusler compounds," SPIN 02, 1230006.

Baibich, M., J.-M. Broto, A. Fert, F. Nguyen Van Dau, F. Petroff, P. Etienne, G. Creuzet, A. Friederich, and J. Chazelas, 1988, "Giant magnetoresistance of (001)Fe/(001)Cr magnetic superlattices," Phys. Rev. Lett. 61, 2472.

Ballhausen, C. J., 1962, Introduction to Ligand Field Theory (McGraw-Hill, New York).

Bandiera, S., et al., 2011, "Spin transfer torque switching assisted by thermally induced anisotropy reorientation in perpendicular magnetic tunnel junctions," Appl. Phys. Lett. 99, 202507.

Barati, E., M. Cinal, D. M. Edwards, and A. Umerski, 2013, "Calculation of Gilbert damping in ferromagnetic films," EPJ Web Conf. 40, 18003

Barnes, S. E., J. Ieda, and S. Maekawa, 2014, "Rashba Spin-Orbit Anisotropy and the Electric Field Control of Magnetism," Sci. Rep. 4, 4105.

Barsukov, I., Y. Fu, A. M. Gonçalves, M. Spasova, M. Farle, R. E. Arias, and I. N. Krivorotov, 2014, "Field-dependent perpendicular magnetic anisotropy in CoFeB thin films," Appl. Phys. Lett. 105, 152403.

Bauer, G. E., W. E. Saitoh, and B. J. Van Wees, 2012, "Spincaloritronics," Nat. Mater. 11, 391-399. 
Bauer, U., M. Przybylski, J. Kirschner, and G. S. D. Beach, 2012, "Magnetoelectric charge trap memory," Nano Lett. 12, 1437.

Bauer, U., L. Yao, A. J. Tan, P. Agrawal, S. Emori, H.-L. Tuller, S. van Dijken, and G. S. D. Beach, 2015, "Magneto-ionic control of interfacial magnetism," Nat. Mater. 14, 174-181.

Bedau, D., H. Liu, J. Bouzaglou, A. D. Kent, J. Z. Sun, J. A. Katine, E. E. Fullerton, and S. Mangin, 2010, "Ultrafast spin transfer switching in spin valve nanopillars with perpendicular anisotropy," Appl. Phys. Lett. 96, 022514.

Beleggia, M., M. De Graef, Y.T. Millev, D. A. Goode, and G. Rowlands, 2005, "Demagnetization factors for elliptic cylinders," J. Phys. D 38, 3333.

Berger, L., 1996, "Emission of spin waves by a magnetic multilayer traversed by a current," Phys. Rev. B 54, 4828-4830.

Berger, L., 1999, "Generation of dc voltages by a magnetic multilayer undergoing ferromagnetic resonance," Phys. Rev. B 59, 11465-11470.

Bernas, H., J.-P. Attané, K. H. Heinig, D. Halley, D. Ravelosona, A. Marty, P. Auric, C. Chappert, and Y. Samson, 2003, "Ordering intermetallic alloys by ion irradiation: A way to tailor magnetic media," Phys. Rev. Lett. 91, 077203.

Bethe, H. A., 1929, "Splitting of Terms in Crystals," Ann. Phys. (Berlin) 395, 133-206.

Binasch, G., P. Grünberg, F. Saurenbach, and W. Zinn, 1989, "Enhanced magnetoresistance in layered magnetic structures with antiferromagnetic interlayer exchange," Phys. Rev. B 39, 4828-4830.

Bloch, F., and G. Gentile, 1931, "Zur Anisotropie der Magnetisierung ferromagnetischer Einkristalle,” Z. Phys. 70, 395.

Blonski, P., and J. Hafner, 2009, "Density-functional theory of the magnetic anisotropy of nanostructures: An assessment of different approximations," J. Phys. Condens. Matter 21, 426001.

Bogdanov, A. N., and U. K. Röszligler, 2001, "Chiral symmetry breaking in magnetic thin films and multilayers," Phys. Rev. Lett. 87, 037203.

Bonell, F., S. Murakami, Y. Shiota, T. Nozaki, T. Shinjo, and Y. Suzuki, 2011, "Large change in perpendicular magnetic anisotropy induced by an electric field in FePd ultrathin films," Appl. Phys. Lett. 98, 232510.

Bonell, F., et al., 2012, "Spin-polarized electron tunneling in bcc $\mathrm{FeCo} / \mathrm{MgO} / \mathrm{FeCo}(001)$ magnetic tunnel junctions," Phys. Rev. Lett. 108, 176602.

Bonell, F., Y. T. Takahashi, D. D. Lam, S. Yoshida, Y. Shiota, S. Miwa, T. Nakamura, and Y. Suzuki, 2013, "Reversible change in the oxidation state and magnetic circular dichroism of Fe driven by an electric field at the $\mathrm{FeCo} / \mathrm{MgO}$ interface," Appl. Phys. Lett. 102, 152401.

Bose, T., R. Cuadrado, R. F. L. Evans, R. V. Chepulskii, D. Apalkov, and R. W. Chantrell, 2016, "First-principles study of the FeMgO (001) interface: magnetic anisotropy," J. Phys. Condens. Matter 28, 156003.

Boulle, O., S. Rohart, L. D. Buda-Prejbeanu, E. Jué, I. M. Miron, S. Pizzini, J. Vogel, G. Gaudin, and A. Thiaville, 2013, "Domain wall tilting in the presence of the Dzyaloshinskii-Moriya interaction in out-of-plane magnetized magnetic nanotracks," Phys. Rev. Lett. 111, 217203.

Boulle, O., et al., 2016, "Room-temperature chiral magnetic skyrmions in ultrathin magnetic nanostructures," Nat. Nanotechnol. 11, 449 .

Brooks, H., "Ferromagnetic Anisotropy and the Itinerant Electron Model,” 1940, Phys. Rev. 58, 909.
Bruno, P., 1989, "Tight-binding approach to the orbital magnetic moment and magnetocrystalline anisotropy of transition-metal monolayers," Phys. Rev. B 39, 865-868(R).

Bruno, P., 1993, "Physical origins and theoretical models of magnetic anisotropy," in Magnetismus von Festkörpern und Grenzflächen, IFF Ferienkurs, edited by P. H. Dederichs, P. Grünberg, and W. Zinns (Forschungszentrum Jülich), pp. 24.1-24.8.

Bruno, P., and J.-P. Renard, 1989, "Magnetic surface anisotropy of transition metal ultrathin films," Appl. Phys. A 49, 499-506.

Butler, W. H., 2008, "Tunneling magnetoresistance from a symmetry filtering effect," Sci. Technol. Adv. Mater. 9, 014106.

Butler, W. H., X. G. Zhang, S. Vutukuri, M. Chshiev, and T.C. Schulthess, 2005, "Theory of tunneling magnetoresistance for epitaxial systems," IEEE Trans. Magn. 41, 2645-2648.

Butler, W. H., X.-G. Zhang, T. C. Schulthess, and J. M. MacLaren, 2001, "Spin-dependent tunneling conductance of FeMgO|Fe sandwiches," Phys. Rev. B 63, 054416.

Carcia, P. F., A. D. Meinhaldt, and A. Suna, 1985, "Perpendicular magnetic anisotropy in Pd/Co thin film layered structures," Appl. Phys. Lett. 47, 178-180.

Carson, G. A., M. H. Nassir, and M. A. Langell, 1996, "Epitaxial growth of $\mathrm{Co}_{3} \mathrm{O}_{4}$ on $\mathrm{CoO}(100)$," J. Vac. Sci. Technol. A 14, 1637-1642.

Chappert, C., and P. Bruno, 1988, "Magnetic anisotropy in metallic ultrathin films and related experiments on cobalt films," J. Appl. Phys. 64, 5736-5741.

Chappert, C., A. Fert, and F. Van Dau, 2007, “The emergence of spin electronics in data storage," Nat. Mater. 6, 813-823.

Chen, X., C. Feng, Z. L. Wu, F. Yang, Y. Liu, S. Jiang, M. H. Li, and G. H. Yu, 2014, "Interfacial oxygen migration and its effect on the magnetic anisotropy in $\mathrm{Pt} / \mathrm{Co} / \mathrm{MgO} / \mathrm{Pt}$ films," Appl. Phys. Lett. 104, 052413.

Chien, C. L., S. H. Liou, B. K. Ha, and K. M. Unruh, 1985, "Rapidly quenched FexTa100-x alloys," J. Appl. Phys. 57, 3539-3541.

Choi, H. S., et al., 2014, "Spin nano-oscillator-based wireless communication," Sci. Rep. 4, 5486.

Chung, S.-W., et al., 2016, "4 Gbit density STT-MRAM using perpendicular MTJ realized with compact cell structure," Electron Devices Meeting (IEDM), 2016 IEEE International, p. 27.1.1.

Cros, V., J. Grollier, M. Munoz Sanchez, A. Fert, and F. Nguyen Van Dau, 2014, "Spintronic device with control by domain wall displacement induced by a current of spin-polarized carriers," Patent US 7969762 B2.

Cubukcu, M.O. Boulle, M. Drouard, K. Garello, C. O. Avci, I. M. Miron, J. Langer, B. Ocker, P. Gambardella, and G. Gaudin, 2014, "Spin-orbit torque magnetization switching of a three-terminal perpendicular magnetic tunnel junction," Appl. Phys. Lett. 104, 042406.

Cuchet, L., B. Rodmacq, S. Auffret, R. C. Sousa, and B. Dieny, 2014, "Influence of magnetic electrodes thicknesses on the transport properties of magnetic tunnel junctions with perpendicular anisotropy," Appl. Phys. Lett. 105, 052408.

Cuchet, L., B. Rodmacq, S. Auffret, R. C. Sousa, I. L. Prejbeanu, and B. Dieny, 2015, "Perpendicular magnetic tunnel junctions with double barrier and single or synthetic antiferromagnetic storage layer," J. Appl. Phys. 117, 233901.

Da Costa, V., A. Iovan, K. Ounadjela, W. Allen, J. F. Gregg, and B. Dieny, 2002, "Spin-polarized electronic reflections at metal-oxide interfaces," J. Magn. Magn. Mater. 240, 140-142.

Daalderop, G. H. O., P. J. Kelly, and F. J. A. den Broeder, 1992, "Prediction and confirmation of perpendicular magnetic anisotropy in Co/Ni multilayers," Phys. Rev. Lett. 68, 682-685. 
Daalderop, G. H. O., P. J. Kelly, and M. F. H. Schuurmans, 1990, "First-principles calculation of the magnetic anisotropy energy of $(\mathrm{Co})_{n} /(X)_{m}$ multilayers," Phys. Rev. B 42, 7270-7272(R).

Daalderop, G. H. O., P. J. Kelly, and M.F. H. Schuurmans, 1994a, "Magnetic anisotropy of a free-standing Co monolayer," Phys. Rev. B 50, 9989-10003.

Daalderop, G. H. O., P. J. Kelly, and M. F. H. Schuurmans, 1994b, "Magnetic anisotropy from first principles," in Ultrathin Magnetic Structures I, edited by J. A. C. Bland and B. Heinrich (Springer, Berlin), pp. 40-64.

Dahmane, Y., C. Arm, S. Auffret, U. Ebels, B. Rodmacq, and B. Dieny, 2009, "Oscillatory behavior of perpendicular magnetic anisotropy in $\mathrm{Pt} / \mathrm{Co} / \mathrm{Al}(\mathrm{Ox})$ films as a function of $\mathrm{Al}$ thickness," Appl. Phys. Lett. 95, 222514.

Den Broeder, F. J. A., H. C. Donkersllot, H. J. G. Draaisma, and W. J. M. de Jonge, 1987, "Magnetic properties and structure of Pd/Co and Pd/Fe multilayers," J. Appl. Phys. 61, 4317-4319.

Den Broeder, F. J. A., W. Hoving, and P. J. H. Bloemen, 1991, "Perpendicular magnetic anisotropy," J. Magn. Magn. Mater. 93, 562-570.

Devolder, T., I. Barisic, S. Eimer, K. Garcia, J.-P. Adam, B. Ockert, and D. Ravelosona, 2013, "Irradiation-induced tailoring of the magnetism of $\mathrm{CoFeB} / \mathrm{MgO}$ ultrathin films," J. Appl. Phys. 113, 203912.

Devolder, T., P.-H. Ducrot, J.-P. Adam, I. Barisic, N. Vernier, J. V. Kim, B. Ockert, and D. Ravelosona, 2013, "Damping of $\mathrm{Co}_{x} \mathrm{Fe}_{80-x} \mathrm{~B}_{20}$ ultrathin films with perpendicular magnetic anisotropy," Appl. Phys. Lett. 102, 022407.

Dieny, B., and O. Redon, 2001, "Magnetic tunnel junction, magnetic device, memory and writing and reading methods using said devices," Patent Application US 6,950,335B2.

Dieny, B., S. Sankar, M. McCartney, D. Smith, P. Bayle-Guillemaud, and A. Berkowitz, 1998, "Spin-polarized tunneling in discontinuous metal/insulator multilayers," J. Magn. Magn. Mater. 185, 283-292.

Dieny, B., V. S. Speriosu, S. S. P. Parkin, B. A. Gurney, D. R. Wilhoit, and D. Mauri, 1991, "Giant magnetoresistance in soft ferromagnetic multilayers," Phys. Rev. B 43, 1297-1300.

Dieny, B., et al., 2010, "Spin-transfer effect and its use in spintronic components," Int. J. Nanotechnology 7, 591-614.

Ding, M., and Poon, S. J., 2012, "Perpendicular magnetization of Co20Fe50Ge30 films induced by MgO interface," Appl. Phys. Lett. 101, 122408.

Du, C., H. Wang, F. Yang, and P. C. Hammel, 2014, "Systematic variation of spin-orbit coupling with d-orbital filling: Large inverse spin Hall effect in 3d transition metals," Phys. Rev. B 90, 140407(R).

Duan, C.-G., J. P. Velev, R. F. Sabirianov, Z. Zhu, J. Chu, S. S. Jaswal, and E. Y. Tsymbal, 2008, "Surface magnetoelectric effect in ferromagnetic metal films," Phys. Rev. Lett. 101, 137201.

Dussaux, A., et al., 2010, "Large microwave generation from currentdriven magnetic vortex oscillators in magnetic tunnel junctions," Nat. Commun. 1, 8.

Dynna, M., J.-L. Vassent, A. Marty, and B. Gilles, 1996, "Low energy electron diffraction investigation of the surface deformation induced by misfit dislocations in thin $\mathrm{MgO}$ films grown on Fe(001)," J. Appl. Phys. 80, 2650-2657.

Dzialoshinskii, I. E., 1957, "Thermodynamic theory of weak ferromagnetism in antiferromagnetic substances," Zh. Eksp. Teor. Fiz. 32, 1547-1562; JETP 5, 1259-1272 (1957) [http://www.jetp.ac.ru/ cgi-bin/e/index/e/5/6/p1259?a=list].

Egelhoff, W. F., P. J. Chen, C. J. Powell, M. D. Stiles, R. D. McMichael, J.H. Judy, K. Takano, A. Berkowitz, and J. M.
Daughton, 1997, "Specular electron scattering in giant magnetoresistance spin valves," IEEE Trans. Magn. 33, 3580-3582.

Emori, S., U. Bauer, S.-M. Ahn, E. Martinez, and G. S. D. Beach, 2013, "Current-driven dynamics of chiral ferromagnetic domain walls," Nat. Mater. 12, 611-616.

Emori, S., D. C. Bono, and G. S. D. Beach, 2012, "Interfacial current-induced torques in $\mathrm{Pt} / \mathrm{Co} / \mathrm{GdOx}$," Appl. Phys. Lett. 101, 042405.

Emori, S., T. Nan, A. M. Belkessam, X. Wang, A. D. Matyushov, C. J. Babroski, Y. Gao, H. Lin, and N. X. Sun, 2016, "Interfacial spin-orbit torque without bulk spin-orbit coupling," Phys. Rev. B 93, 180402(R).

Endo, M., S. Kanai, S. Ikeda, F. Matsukura, and H. Ohno, 2010, "Electric-field effects on thickness dependent magnetic anisotropy of sputtered MgO/Co 40 Fe 40 B 20 / Ta structures," Appl. Phys. Lett. 96, 212503.

Fabian, J., and S. Das Sarma, 1999, "Spin relaxation of conduction electrons,” J. Vac. Sci. Technol. B 17, 1708-1715.

Farle, M., 1998, "Ferromagnetic resonance of ultrathin metallic layers,” Rep. Prog. Phys. 61, 755.

Faure-Vincent, J., et al., 2003, "High tunnel magnetoresistance in epitaxial $\mathrm{Fe} / \mathrm{MgO} / \mathrm{Fe}$ tunnel juncitons," Appl. Phys. Lett. 82, 4507.

Fert, A., V. Cros, and J. Sampaio, 2013, "Skyrmions on the track," Nat. Nanotechnol. 8, 152-156.

Fuchs, G. D., N. C. Emley, I. N. Krivorotov, P. M. Braganca, E. M. Ryan, S. I. Kiselev, J. C. Sankey, D. C. Ralph, and R. A. Buhrman, 2004, "Spin-transfer effects in nanoscale magnetic tunnel junctions," Appl. Phys. Lett. 85, 1205-1207.

Gabor, M.S., T. Petrisor, Jr., C. Tiusan, and T. Petrisor, 2013, "Perpendicular magnetic anisotropy in $\mathrm{Ta} / \mathrm{Co}_{2} \mathrm{FeAl} / \mathrm{MgO}$ multilayers,” J. Appl. Phys. 114, 063905.

Gambardella, P., and I. M. Miron, 2011, "Current-induced spin-orbit torques,” Phil. Trans. R. Soc. A 369, 3175-3197.

Garello, K., I. M. Miron, C. O. Avci, F. Freimuth, Y. Mokrousov, S. Blügel, S. Auffret, O. Boulle, G. Gaudin, and P. Gambardella, 2013, "Symmetry and magnitude of spin-orbit torques in ferromagnetic heterostructures," Nat. Nanotechnol. 8, 587-593.

Gaudin, G., I. M. Miron, P. Gambardella, and A. Schuhl, 2010, "A writable magnetic memory element," Patent application US 2012020152A1; “A writable magnetic element," Patent applications US 2012018822A1, US 2012098077A1.

Gay, J. G., and R. Richter, 1986, "Spin anisotropy of ferromagnetic films," Phys. Rev. Lett. 56, 2728-2731.

Gehanno, V., A. Marty, B. Gilles, and Y. Samson, 1997, "Magnetic domains in epitaxial ordered FePd(001) thin films with perpendicular magnetic anisotropy," Phys. Rev. B 55, 12552-12555.

Giannozzi, P., et al., 2009, "QUANTUM ESPRESSO: a modular and open-source software project for quantum simulations of materials," J. Phys. Condens. Matter 21, 395502.

Gottwald, M., et al., 2012, "Co/Ni(111) superlattices studied by microscopy, X-ray absorption, and ab initio calculations," Phys. Rev. B 86, 014425.

Grimbolt, J., J.-P. Bonnelle, and J.-P. Beaufils, 1976, "ESCA study on cobalt and molybdenum deposited on alumina-quantitative analysis of recovery and states of specific cation absorption," J. Electron Spectrosc. Relat. Phenom. 8, 437-447.

Guoa, V. W., B. Lu, X. Wu, G. Ju, B. Valcu, and D. Weller, 2006, "A survey of anisotropy measurement techniques and study of thickness effect on interfacial and volume anisotropies in $\mathrm{Co} / \mathrm{Pt}$ multilayer media," J. Appl. Phys. 99, 08E918.

Ha, S.-S., N.-H. Kim, S. Lee, C.-Y. You, Y. Shiota, T. Maruyama, T. Nozaki, and Y. Suzuki, 2010, "Voltage induced magnetic 
anisotropy change in ultrathin $\mathrm{Fe}_{80} \mathrm{Co}_{20} / \mathrm{MgO}$ junctions with Brillouin light scattering," Appl. Phys. Lett. 96, 142512.

Hallal, A., B. Dieny, and M. Chshiev, 2014, "Impurity-induced enhancement of perpendicular magnetic anisotropy in $\mathrm{Fe} / \mathrm{MgO}$ tunnel junctions," Phys. Rev. B 90, 064422.

Hallal, A., H. X. Yang, B. Dieny, and M. Chshiev, 2013, "Anatomy of perpendicular magnetic anisotropy in $\mathrm{Fe} / \mathrm{MgO}$ magnetic tunnel junctions: First-principles insight," Phys. Rev. B 88, 184423.

Heinonen, O. G., and D. V. Dimitrov, 2010, "Switching-current reduction in perpendicular-anisotropy spin torque magnetic tunnel junctions," J. Appl. Phys. 108, 014305.

Heinze, S., K. von Bergmann, M. Menzel, J. Brede, A. Kubetzka, R. Wiesendanger, G. Bihlmayer, and S. Blugel, 2011, "Spontaneous atomic-scale magnetic skyrmion lattice in two dimensions," Nat. Phys. 7, 713-718.

Hrabec, A., N. A. Porter, A. Wells, M. J. Benitez, G. Burnell, S. McVitie, D. McGrouther, T.A. Moore, and C.H. Marrows, 2014, "Measuring and tailoring the Dzyaloshinskii-Moriya interaction in perpendicularly magnetized thin films," Phys. Rev. B 90, 020402(R).

Hu, G., et al., 2015, "STT-MRAM with double magnetic tunnel junctions," Electron Devices Meeting (IEDM), 2015 IEEE International, p. 26.3.1.

Huai, Y., 2008, "Spin transfer torque MRAM (STT-MRAM): Challenges and prospects," A.A.P.P.S. Bull. 18, 33-40.

Huai, Y., F. Albert, P. Nguyen, M. Pakala, and T. Valet, 2004, "Observation of spin-transfer switching in deep submicron-sized and low-resistance magnetic tunnel junctions," Appl. Phys. Lett. 84, 3118-3120.

Huang, R. T., F. R. Chen, J. J. Kai, I. F. Tsu, S. Mao, and W. Kai, 2001, "Diffusion behavior of the spin valve structure," J. Appl. Phys. 89, 7625-7627.

Ibrahim, F., A. Hallal, B. Dieny, and M. Chshiev, 2016a, "Giant variation of the perpendicular magnetic anisotropy at $\mathrm{Fe} / \mathrm{MgO}$ interfaces by oxygen migration: a first-principles study," arXiv:1610.08859

Ibrahim, F., H. X. Yang, A. Hallal, B. Dieny, and M. Chshiev, 2016b, "Anatomy of electric field control of perpendicular magnetic anisotropy at Fe/MgO interfaces," Phys. Rev. B 93, 014429.

Iihama, S., S. Mizukami, H. Naganuma, M. Oogane, Y. Ando, and T. Miyazaki, 2014, "Gilbert damping constants of $\mathrm{Ta} / \mathrm{CoFeB} / \mathrm{MgO}$ (Ta) thin films measured by optical detection of precessional magnetization dynamics," Phys. Rev. B 89, 174416.

Ikeda, S., J. Hayakawa, Y. Ashizawa, Y. M. Lee, K. Miura, H. Hasegawa, M. Tsunoda, F. Matsukura, and H. Ohno, 2008, “Tunnel magnetoresistance of $604 \%$ at $300 \mathrm{~K}$ by suppression of Ta diffusion in $\mathrm{CoFeB} / \mathrm{MgO} / \mathrm{CoFeB}$ pseudo-spin-valves annealed at high temperature," Appl. Phys. Lett. 93, 082508.

Ikeda, S., J. Hayakawa, Y. M. Lee, R. Sasaki, T. Meguro, F. Matsukura, and H. Ohno, 2005, "Dependence of tunnel magnetoresistance in $\mathrm{MgO}$ based magnetic tunnel junctions on Ar pressure during MgO sputtering," Jpn. J. Appl. Phys. 44, L1442-L1445.

Ikeda, S., K. Miura, H. Yamamoto, K. Mizunuma, H. D. Gan, M. Endo, S. Kanai, J. Hayakawa, F. Matsukura, and H. Ohno, 2010, "A perpendicular-anisotropy $\mathrm{CoFeB}-\mathrm{MgO}$ magnetic tunnel junction," Nat. Mater. 9, 721-724.

ITRS, 2010, http://www.itrs.net/Links/2010ITRS/2010Update/ToPost/ ERD_ERM_2010FINALReportMemory Assessment_ITRS.pdf.

Iwasaki, J., M. Mochizuki, and N. Nagaosa, 2013, "Current-induced skyrmion dynamics in constricted geometries," Nat. Nanotechnol. 8, 742-747.

Jan, G., Y.-J. Wang, T. Moriyama, Y.-J. Lee, M. Lin, T. Zhong, R.-Y. Tong, T. Torng, and P.-K. Wang, 2012, "High spin torque efficiency of magnetic tunnel junctions with $\mathrm{MgO} / \mathrm{CoFeB} / \mathrm{MgO}$ free layer," Appl. Phys. Express 5, 093008.

Jansen, H. J. F., "Magnetic anisotropy in density-functional theory," 1988, Phys. Rev. B 38, 8022.

Je, S. G., D. H. Kim, S. C. Yoo, B. C. Min, K. J. Lee, and S. B. Choe, 2013, "Asymmetric magnetic domain-wall motion by the Dzyaloshinskii-Moriya interaction,” Phys. Rev. B 88, 214401.

Jiang, W., et al., 2015, "Blowing magnetic skyrmion bubbles," Science 349, 283-286.

Johnson, M. T., P. J. H. Bloemen, F. J. A. den Broeder, and J. J. de Vries, 1996, "Magnetic anisotropy in metallic multilayers," Rep. Prog. Phys. 59, 1409-1458.

Johnson, M. T., R. Jungblut, P. J. Kelly, and F. J. A. den Broeder, 1995, "Perpendicular magnetic anisotropy of multilayers: Recent insights," J. Magn. Magn. Mater. 148, 118-124.

Jones, W., and N. H. March, "Theoretical Solid State Physics: Perfect lattices in equilibrium," 1973, John Wiley and Sons Ltd, New York.

Kambersky, V., 1976, "On ferromagnetic resonance damping in metals," Czech. J. Phys. B 26, 1366-1383.

Kanai, S., M. Tsujikawa, Y. Miura, M. Shirai, F. Matsukura, and H. Ohno, 2014, "Magnetic anisotropy in $\mathrm{Ta} / \mathrm{CoFeB} / \mathrm{MgO}$ investigated by x-ray magnetic circular dichroism and first-principles calculation,” Appl. Phys. Lett. 105, 222409.

Karthik, S. V., Y. K. Takahashi, T. Ohkubo, K. Hono, H. D. Gan, S. Ikeda, and H. Ohno, 2012, "Transmission electron microscopy study on the effect of various capping layers on $\mathrm{CoFeB} / \mathrm{MgO}$ / $\mathrm{CoFeB}$ pseudo spin valves annealed at different temperatures," J. Appl. Phys. 111, 083922.

Katine, J. A., F. J. Albert, R. A. Buhrman, E. B. Myers, and D. C. Ralph, 2000, "Current-driven magnetization reversal and spinwave excitations in $\mathrm{Co} / \mathrm{Cu} / \mathrm{Co}$ pillars," Phys. Rev. Lett. 84, 3149-3152.

Kato, T., Y. Matsumoto, S. Kashima, S. Okamoto, N. Kituchi, S. Iwata, O. Kitakami, and S. Tsunashima, 2012, "Perpendicular anisotropy and Gilbert damping in sputtered Co/Pd multilayers," IEEE Trans. Magn. 48, 3288-3291.

Kawahara, T., 2011, "Scalable spin-transfer torque RAM technology for normally-off computing," IEEE Design \& Test of Computers 28, 52-63.

Kent, A. D., B. Ozyilmaz, and E. del Barco, 2004, "Spin-transferinduced precessional magnetization reversal," Appl. Phys. Lett. 84, 3897-3899.

Khomski, D., 2014, "Transition metal compounds" (Cambridge University Press, Cambridge, England).

Khoo, K. H., G. Wu, M. H. Jhon, M. Tran, F. Ernult, K. Eason, H. J. Choi, and C. K. Gan, 2013, "First-principles study of perpendicular magnetic anisotropy in $\mathrm{CoFe} / \mathrm{MgO}$ and $\mathrm{CoFe} / \mathrm{Mg} 3 \mathrm{~B} 2 \mathrm{O} 6$ interfaces," Phys. Rev. B 87, 174403.

Khvalkovskiy, A. V., et al., 2013, "Basic principles of STT-MRAM cell operation in memory arrays," J. Phys. D 46, 074001.

Kim, J., J. Sinha, M. Hayashi, M. Yamanouchi, S. Fukami, T. Suzuki, S. Mitani, and H. Ohno, 2013, "Layer thickness dependence of the current-induced effective field vector in $\mathrm{Ta} / \mathrm{CoFeB} / \mathrm{MgO}$," Nat. Mater. 12, 240-245.

Kim, W., et al., 2011, "Extended scalability of perpendicular STTMRAM toward sub-20 nm MTJ node," Electron Devices Meeting (IEDM), 2011 IEEE International, 24.1.1-24.1.4.

Kishi, T., et al., 2008, "Lower-current and fast switching of a perpendicular TMR for high speed and high density spin-transfer-torque MRAM," Electron Devices Meeting (IEDM), 2008 IEEE International, p. 1.

Kita, K., D. W. Abraham, M. J. Gajek, and D. C. Worledge, 2012, "Electric-field control of magnetic anisotropy of 
Co0.6Fe0.2B0.2/oxide stacks using reduced voltage," J. Appl. Phys. 112, 033919.

Koch, R., 1997, "Intrinsic stress of epitaxial thin films and surface layers," in Growth and Properties of Ultrathin Epitaxial Layers, edited by O. A. King and D. P. Woodruff (Elsevier Science, New York), Chap. 12, pp. 448-489.

Koch, R. H., J. A. Katine, and J.Z. Sun, 2004, "Time-resolved reversal of spin transfer switching in a nanomagnet," Phys. Rev. Lett. 92, 088302.

Koo, J. W., S. Mitani, T. T. Sasaki, H. Sukegawa, Z. C. Wen, T. Ohkubo, T. Niizeki, K. Inomata, and K. Hono, 2013, "Large perpendicular magnetic anisotropy at $\mathrm{Fe} / \mathrm{MgO}$ interface," Appl. Phys. Lett. 103, 192401.

Kozina, X., S. Ouardi, B. Balke, G. Stryganyuk, G. H. Fecher, C. Felser, S. Ikeda, H. Ohno, and E. Ikenaga, 2010, "A non-destructive analysis of the $\mathrm{B}$ diffusion in $\mathrm{Ta} / \mathrm{CoFeB} / \mathrm{MgO} / \mathrm{CoFeB} / \mathrm{Ta}$ magnetic tunnel junctions by hard X-ray photoemission," Appl. Phys. Lett. 96, 072105.

Koziol-Rachwal, A., T. Slezak, P. Kuzwik, M. Urbaniak, F. Stobiescki, L. D. Yao, S. van Dijken, and J. Koerchi, 2014, "Tunable magnetic properties of monoatomic metal-oxide Fe/ MgO multilayers," Phys. Rev. B 90, 045428.

Koziol-Rachwal, A., et al., 2013, "Room-temperature perpendicular magnetic anisotropy of $\mathrm{MgO} / \mathrm{Fe} / \mathrm{MgO}$ ultrathin films," J. Appl. Phys. 114, 224307.

Kubota, H., S. Ishibashi, T. Saruya, T. Nozaki, A. Fukushima, K. Yakushiji, K. Ando, Y. Suzuki, and S. Yuasa, 2012, "Enhancement of perpendicular magnetic anisotropy in FeB free layers using a thin MgO cap layer,' J. Appl. Phys. 111, $07 \mathrm{C} 723$.

Kyuno, K., R. Yamamoto, and S. Asano, 1992, "Theoretical study on the magnetocrystalline anisotropy of $\mathrm{X} / \mathrm{Co}(\mathrm{X}=\mathrm{Pd}, \mathrm{Pt}, \mathrm{Cu}, \mathrm{Ag}, \mathrm{Au})$ multilayers," J. Phys. Soc. Jpn. 61, 2099-2113.

Lambert, C. H., A. Rajanikanth, T. Hauet, S. Mangin, and E. E. Fullerton, 2013, "Quantifying perpendicular magnetic anisotropy at the $\mathrm{Fe} / \mathrm{MgO}(001)$ interface," Appl. Phys. Lett. 102, 122410 .

Lee, D. S., H. T. Chang, C.W. Cheng, and G. Chern, 2014, "Perpendicular magnetic anisotropy in $\mathrm{MgO} / \mathrm{CoFeB} / \mathrm{Nb}$ and a comparison of the cap layer effect," IEEE Trans. Magn. 50, 3201904.

Lee, H., Y. H. A. Wang, C. K. A. Mewes, W. H. Butler, T. Mewes, S. Maat, B. York, M. J. Carey, and J. R. Childress, 2009, "Magnetization relaxation and structure of $\mathrm{CoFeGe}$ alloys," Appl. Phys. Lett. 95, 082502 .

Lee, K., J. J. Sapan, S.H. Kang, and E. E. Fullerton, 2011, "Perpendicular magnetization of $\mathrm{CoFeB}$ on single crystal MgO," J. Appl. Phys. 109, 123910.

Lee, K. J., O. Redon, and B. Dieny, 2005, "Analytical investigation of spin-transfer dynamics using a perpendicular-to-plane polarizer," Appl. Phys. Lett. 86, 022505.

Lee, O. J., V. S. Pribiag, P. M. Braganca, P. G. Gowtham, D. C. Ralph, and R.A. Buhrman, 2009, "Ultrafast switching of a nanomagnet by a combined out-of-plane and in-plane polarized spin-current pulse," Appl. Phys. Lett. 95, 012506.

Lee, S.-C., K.-S. Kim, S.-H. Lee, U.-H. Pi, K. Kim, Y. Jang, and Y. I. Chung, 2013, "Effect of Fe-O distance on magnetocrystalline anisotropy energy at the $\mathrm{Fe} / \mathrm{MgO}(001)$ interface," J. Appl. Phys. 113, 023914.

Lee, Y. M., J. Hayakawa, S. Ikeda, F. Matsukura, and H. Ohno, 2006, "Giant tunnel magnetoresistance and high annealing stability in $\mathrm{CoFeB} / \mathrm{MgO} / \mathrm{CoFeB}$ magnetic tunnel junctions with synthetic pinned layer," Appl. Phys. Lett. 89, 042506.
Lessard, A., T. H. Moos, and W. Hübner, 1997, "Magnetocrystalline anisotropy energy of transition-metal thin films: A nonperturbative theory," Phys. Rev. B 56, 2594.

Li, D., C. Barreteau, and A. Smogunov, 2016, "Magnetocrystalline anisotropy of $\mathrm{Fe}$ and $\mathrm{Co}$ slabs and clusters on $\mathrm{SrTiO}_{3}$ by firstprinciples," Phys. Rev. B 93, 144405.

Li, D., A. Smogunov, C. Barreteau, F. Ducastelle, and D. Spanjaard, 2013, "Magnetocrystalline anisotropy energy of $\mathrm{Fe}(001)$ and $\mathrm{Fe}$ (110) slabs and nanoclusters: A detailed local analysis within a tight-binding model," Phys. Rev. B 88, 214413.

Li, Z., and S. Zhang, 2004, "Thermally assisted magnetization reversal in the presence of a spin-transfer torque," Phys. Rev. B 69, 134416.

Liang, S.-H., et al., 2014, "Large and robust electrical spin injection into GaAs at zero magnetic field using an ultrathin $\mathrm{CoFeB} / \mathrm{MgO}$ injector," 2014, Phys. Rev. B 90, 085310.

Lide, D. R., 2000, Handbook of Chemistry and Physics (CRC, Boca Raton), 81st ed., Sec. 5.

Lin, W., N. Vernier, G. Agnus, K. Garcia, B. Ocker, W. Zhao, E. E. Fullerton, and D. Ravelosona, 2016, "Universal domain wall dynamics under electric field in $\mathrm{Ta} / \mathrm{CoFeB} / \mathrm{MgO}$ devices with perpendicular anisotropy," Nat. Commun. 7, 13532.

Liu, L., O. J. Lee, T. J. Gudmundsen, D. C. Ralph, and R. A. Buhrman, 2012, "Current-induced switching of perpendicularly magnetized magnetic layers using spin torque from the spin Hall effect," Phys. Rev. Lett. 109, 096602.

Liu, L., C. F. Pai, Y. Li, H. W. Tseng, D. C. Ralph, and R. A. Buhrman, 2012, "Spin-torque switching with the giant spin Hall effect of tantalum," Science 336, 555-558.

Liu, T., J. W. Cai, and L. Sun, 2012, "Large enhanced perpendicular magnetic anisotropy in $\mathrm{CoFeB} / \mathrm{MgO}$ system with the typical $\mathrm{Ta}$ buffer replaced by an Hf layer," AIP Adv. 2, 032151.

Liu, T., Y. Zhang, J. W. Cai, and H. Y. Pan, 2014, “Thermally robust $\mathrm{Mo} / \mathrm{CoFeB} / \mathrm{MgO}$ trilayers with strong perpendicular magnetic anisotrop," Sci. Rep. 4, 5895.

Liu, X., W. Zhang, M. J. Carter, and G. Xiao, 2011, "Ferromagnetic resonance and damping properties of $\mathrm{CoFeB}$ thin films as free layers in MgO-based magnetic tunnel junctions," J. Appl. Phys. 110, 033910.

Lu, Y., et al., 2012, "Spin-orbit coupling effect by minority interface resonance states in single-crystal magnetic tunnel junctions," Phys. Rev. B 86, 184420.

Ma, Q. L., S. Lihama, T. Kubota, X. M. Zhang, S. Mizukami, Y. Ando, and T. Miyazaki, 2012, "Effect of Mg interlayer on perpendicular magnetic anisotropy of $\mathrm{CoFeB}$ films in $\mathrm{MgO} / \mathrm{Mg}$ / CoFeB/Ta structure," Appl. Phys. Lett. 101, 122414.

Manchon, A., and S. Zhang, 2008, "Theory of nonequilibrium intrinsic spin torque in a single nanomagnet," Phys. Rev. B 78, 212405.

Manchon, A., and S. Zhang, 2009, "Theory of spin torque due to spin-orbit coupling," Phys. Rev. B 79, 094422.

Manchon, A., et al., 2008, "Analysis of anisotropy crossover due to oxygen in Pt/Co/MOx trilayers," J. Appl. Phys. 104, 043914.

Maruyama, T., et al., 2009, "Large voltage-induced magnetic anisotropy change in a few atomic layers of iron," Nat. Nanotechnol. 4, 158-161.

Mathon, J., and A. Umerski, 2001, "Theory of tunneling magnetoresistance of an epitaxial $\mathrm{Fe} / \mathrm{MgO} / \mathrm{Fe}(001)$ junction," Phys. Rev. B 63, 220403.

Mattheiss, L. F., 1972, "Energy Bands for KNiF3, SrTiO3, KMoO3, and KTaO3," Phys. Rev. B 6, 4718.

Meiklejohn, W.H., 1962, "Exchange Anisotropy-A Review," J. Appl. Phys. 33, 1328. 
Meyerheim, H. L., R. Popescu, J. Kirschner, N. Jedrecy, M. SauvageSimkin, B. Heinrich, and R. Pinchaux, 2001, "Geometrical and compositional structure at metal-oxide interfaces: $\mathrm{MgO}$ on Fe(001),” Phys. Rev. Lett. 87, 076102.

Min, T., et al., 2010, "A study of write margin of spin torque transfer magnetic random access memory technology," IEEE Trans. Magn. 46, $2322-2327$.

Miron, I. M., K. Garello, G. Gaudin, P.-J. Zermatten, M. V. Costache, S. Auffret, S. Bandiera, B. Rodmacq, A. Schuhl, and P. Gambardella, 2011, "Perpendicular switching of a single ferromagnetic layer induced by in-plane current injection," Nature (London) 476, 189-193.

Miron, I. M., G. Gaudin, S. Auffret, B. Rodmacq, A. Schuhl, S. Pizzini, J. Vogel, and P. Gambardella, 2010, "Current-driven spin torque induced by the Rashba effect in a ferromagnetic metal layer," Nat. Mater. 9, 230-234.

Miron, I. M., P.-J. Zermatten, G. Gaudin, S. Auffret, B. Rodmacq, and A. Schuhl, 2009, "Domain wall spin torque meter," Phys. Rev. Lett. 102, 137202.

Miron, I. M., et al., 2011, "Fast current-induced domain-wall motion controlled by the Rashba effect," Nat. Mater. 10, 419-423.

Miwa, S., et al., 2014, "Highly sensitive nanoscale spin-torque diode," Nat. Mater. 13, 50-56.

Miyakawa, N., D. C. Worledge, and K. Kita, 2013, "Impact of Ta diffusion on the perpendicular magnetic anisotropy of $\mathrm{Ta} / \mathrm{CoFeB} /$ MgO,” IEEE Magn. Lett. 4, 1000104.

Miyazaki, T., and N. Tezuka, 1995, "Giant magnetic tunneling effect in $\mathrm{Fe} / \mathrm{Al}_{2} \mathrm{O}_{3} / \mathrm{Fe}$ junction," J. Magn. Magn. Mater. 139, L231-L234.

Monso, S., B. Rodmacq, S. Auffret, G. Casali, F. Fettar, B. Gilles, B. Dieny, and P. Boyer, 2002, "Crossover from in-plane to perpendicular anisotropy in $\mathrm{Pt} / \mathrm{CoFe} / \mathrm{AlOx}$ as a function of the Al degree of oxidation: A very accurate control of the oxidation of tunnel barrier," Appl. Phys. Lett. 80, 4157-4159.

Moodera, J. S., L. R. Kinder, T. M. Wong, and R. Meservey, 1995, "Large magnetoresistance at room temperature in ferromagnetic thin film tunnel junctions," Phys. Rev. Lett. 74, 3273-3276.

Moriya, T., 1960, "Anisotropic superexchange interaction and weak ferromagnetism," Phys. Rev. 120, 91-98.

Mouillon, A., et al., 2016 (to be published).

Mühlbauer, S., et al., 2009, "Skyrmion lattice in a chiral magnet," Science 323, 915-919.

Mukherjee, S.S., D. Mac Mahon, F. Bai, C. L. Lee, and S. K. Kurinec, 2009, "Study of boron diffusion in $\mathrm{MgO}$ in $\mathrm{CoFeB}-\mathrm{MgO}$ film stacks using parallel electron energy loss spectroscopy," Appl. Phys. Lett. 94, 082110.

Nakamura, K., T. Akiyama, T. Ito, M. Weinert, and A. J. Freeman, 2010, "Role of an interfacial $\mathrm{FeO}$ layer in the electric-field-driven switching of magnetocrystalline anisotropy at the $\mathrm{Fe} / \mathrm{MgO}$ interface," Phys. Rev. B 81, 220409(R).

Nakamura, K., R. Shimabukuro, Y. Fujiwara, T. Akiyama, T. Ito, and A. J. Freeman, 2009a, "Giant modification of the magnetocrystalline anisotropy in transition-metal monolayers by an external electric field," Phys. Rev. Lett. 102, 187201.

Nakamura, K., R. Shimabukuro, Y. Fujiwara, T. Akiyama, T. Ito, and A.J. Freeman, 2009b, "Origin of electric-field-induced modification of magnetocrystalline anisotropy at $\mathrm{Fe}(001)$ surfaces: Mechanism of dipole formation from first principles," Phys. Rev. B 80, 172402.

Natarajarathinam, A., Z. R. Tadisina, T. Mewes, S. Watts, E. Chen, and S. Gupta, 2012, "Influence of capping layers on CoFeB anisotropy and damping," J. Appl. Phys. 112, 053909.
Néel, L., 1954, "Anisotropie magnétique superficielle et surstructures d'orientation," J. Phys. Radium 15, 225-239.

Nguyen, P. P, and Y. Huai, 2004, "Spin transfer magnetic element with free layers having high perpendicular anisotropy and in-plane equilibrium magnetization," Patent application US 7,531,882B2.

Niranjan, M. K., C.-G. Duan, S. S. Jaswal, and E. Y. Tsymbal, 2010, "Electric field effect on magnetization at the $\mathrm{Fe} / \mathrm{MgO}(001)$ interface,” Appl. Phys. Lett. 96, 222504.

Nistor, L. E., B. Rodmacq, S. Auffret, and B. Dieny, 2009, "Pt/Co/ oxide and oxide/Co/Pt electrodes for perpendicular magnetic tunnel junctions," Appl. Phys. Lett. 94, 012512.

Nistor, L. E., B. Rodmacq, C. Ducruet, C. Portemont, I. L. Prejbeanu, and B. Dieny, 2010, "Correlation between perpendicular anisotropy and magnetoresistance in magnetic tunnel junctions," IEEE Trans. Magn. 46, 1412-1415.

Nogues, J., and I. K. Schuller, 1999, "Exchange bias," J. Magn. Magn. Mater. 192, 203.

Nowak, J. J., et al., 2011, "Demonstration of ultralow bit error rates for spin-torque magnetic random access memory with perpendicular anisotropy," IEEE Magn. Lett. 2, 3000204.

Nozaki, T., Y. Shiota, T. Shiraishi, T. Shinjo, and Y. Suzuki, 2010, "Voltage-induced perpendicular magnetic anisotropy in magnetic tunnel junctions," Appl. Phys. Lett. 96, 022506.

Oh, Y. W., K. D. Lee, J. R. Jeong, and B. G. Park, 2014, "Interfacial perpendicular magnetic anisotropy in $\mathrm{CoFeB} / \mathrm{MgO}$ structure with various underlayers," J. Appl. Phys. 115, 17 C724.

Okabayashi, J., J. W. Koo, H. Sukegawa, S. Mitani, Y. Takagi, and T. Yokoyama, 2014, Appl. Phys. Lett. 105, 122408.

Ortiz Pauyac, C., X. Wang, M. Chshiev, and A. Manchon, 2013, "Angular dependence and symmetry of Rashba spin torque in ferromagnetic heterostructures," Appl. Phys. Lett. 102, 252403.

Pai, C.-F., L. Liu, Y. Li, H. W. Tseng, D. C. Ralph, and R. A. Buhrman, 2012, "Spin transfer torque devices utilizing the giant spin Hall effect of tungsten,” Appl. Phys. Lett. 101, 122404.

Papusoi, C., B. Delaët, B. Rodmacq, D. Houssameddine, J.-P. Michel, U. Ebels, R. C. Sousa, L. D. Buda-Prejbeanu, and B. Dieny, 2009, "100 ps precessional spin transfer switching of a planar magnetic random access memory cell with perpendicular spin polarizer," Appl. Phys. Lett. 95, 072506.

Park, J. G., T. H. Shim, K. S. Chae, D. Y. Lee, Y. Takemura, S. E. Lee, M. S. Jeon, J. U. Baek, S. O. Park, and J. P. Hong, 2014, "Challenging issues for Terra-bit level perpendicular STT-MRAM," Electron Devices Meeting, (IEDM), 2014 IEEE International, pp. 19.2.1-19.2.4.

Parkin, S. S. P., M. Hayashi, and L. Thomas, 2008, "Magnetic domain-wall racetrack memory," Science 320, 190-194.

Parkin, S. S. P., C. Kaiser, A. Panchula, P. M. Rice, B. Hughes, M. Samant, and S. H. Yang, 2004, "Giant tunnel magnetoresistance at room temperature with $\mathrm{MgO}(100)$ tunnel barriers," Nat. Mater. 3, 862-867.

Parkin, S. S. P., N. More, and K. P. Roche, 1990, "Oscillations in exchange coupling and magnetoresistance in metallic structures: $\mathrm{Co} / \mathrm{Ru}, \mathrm{Co} / \mathrm{Cr}$ and Fe/Cr," Phys. Rev. Lett. 64, 2304-2307.

Peng, S.Z., et al., 2015, "Origin of interfacial perpendicular magnetic anisotropy in $\mathrm{MgO} / \mathrm{CoFe} /$ metallic capping layer structures," Sci. Rep. 5, 18173.

Pesin, D. A., and A. H. MacDonald, 2012, "Quantum kinetic theory of current-induced torques in Rashba ferromagnets," Phys. Rev. B 86, 014416.

Pi, U.H., et al., 2010, "Tilting of the spin orientation induced by Rashba effect in ferromagnetic metal layer," Appl. Phys. Lett. 97, 162507. 
Piramanayagama, S. N., 2007, "Perpendicular recording media for hard disk drives," J. Appl. Phys. 102, 011301.

Prejbeanu, I. L., M. Kerekes, R. C. Sousa, H. Sibuet, O. Redon, B. Dieny, and J.-P. Nozières, 2007, "Thermally Assisted MRAM," J. Phys. Condens. Matter 19, 165218.

Prinz, G. A., 1985, "Stabilization of bcc Co via epitaxial growth on GaAs,” Phys. Rev. Lett. 54, 1051-1054.

Rajanikanth, A., T. Hauet, F. Montaigne, S. Mangin, and S. Andrieu, 2013, "Magnetic anisotropy modified by electric field in V/Fe/ $\mathrm{MgO}(001) / \mathrm{Fe}$ epitaxial magnetic tunnel junction," Appl. Phys. Lett. 103, 062402.

Rantschler, J. O., R. D. McMichael, A. Castillo, A. J. Shapiro, J. W. F. Egelhoff, B. B. Maranville, D. Pulugurtha, A. P. Chen, and L. M. Connors, 2007, "Effect of 3d, 4d, and 5d transition metal doping on damping in permalloy thin films," J. Appl. Phys. 101, 033911.

Ravelosona, D., C. Chappert, V. Mathet, and H. Bernas, 2000, "Chemical order induced by ion irradiation in $\mathrm{FePt}(001)$ films," Appl. Phys. Lett. 76, 236-238.

Redon, O., B. Dieny, and B. Rodmacq, 2000, "Magnetic spin polarization and magnetization rotation device with memory and writing process using such device," Patent Application US $6,532,164 \mathrm{~B} 2$.

Reig, C., S. Cardoso de Freitas, and S. C. Mukhopadhyay, 2014, Eds., Giant Magnetoresistance (GMR) Sensors: From Basis to State-of-the-Art Applications (Springer, New York).

Rippard, W. H., M. R. Pufall, S. Kaka, T. J. Silva, S. E. Russek, and J. A. Katine, 2005, "Injection locking and phase control of spin transfer nano-oscillators," Phys. Rev. Lett. 95, 067203.

Rizzo, N. D., et al., 2013, "A fully functional 64 Mb DDR3 ST-MRAM built on $90 \mathrm{~nm}$ CMOS technology," IEEE Trans. Magn. 49, 4441-4446.

Rodmacq, B., S. Auffret, B. Dieny, S. Monso, and P. Boyer, 2003, "Crossovers from in-plane to perpendicular anisotropy in magnetic tunnel junctions as a function of the barrier degree of oxidation," J. Appl. Phys. 93, 7513-7515.

Rodmacq, B., S. Auffret, B. Dieny, and L. E. Nistor, 2008, “Threelayer magnetic element, magnetic field sensor, magnetic memory and magnetic logic gate using such an element," Patent Application US 8,513,944B2.

Rodmacq, B., and B. Dieny, 2006, "Thin-layered magnetic device with high spin polarization perpendicular to the plane of the layers, and magnetic tunnel junction and spin valve using such a device," Patent Application US 7,813,202B2.

Rodmacq, B., A. Manchon, C. Ducruet, S. Auffret, and B. Dieny, 2009, "Influence of thermal annealing on the magnetic properties of Pt/Co/AlOx trilayers," Phys. Rev. B 79, 024423.

Roessler, U. K., A. N. Bogdanov, and C. Pfleiderer, 2006, "Spontaneous skyrmion ground states in magnetic metals," Nature (London) 442, 797-801.

Rossnagel, S. M., and H. Kim, 2003, "Diffusion barrier properties of very thin TaN with high nitrogen concentration," J. Vac. Sci. Technol. B 21, 2550-2554.

Saitoh, E., M. Ueda, H. Miyajima, and G. Tatara, 2006, "Conversion of spin current into charge current at room temperature: Inverse spin-Hall effect," Appl. Phys. Lett. 88, 182509.

Sampaio, J., V. Cros, S. Rohart, A. Thiaville, and A. Fert, 2013, "Nucleation, stability and current-induced motion of isolated magnetic skyrmions in nanostructures," Nat. Nanotechnol. 8, 839-844.

Sankar, S., B. Dieny, and A. Berkowitz, 1997, "Spin-polarized tunnelling in discontinuous $\mathrm{CoFe} / \mathrm{HfO} 2$ multilayers," J. Appl. Phys. 81, 5512-5514.
Sato, H., T. Yamamoto, M. Yamanouchi, S. Ikeda, S. Fukami, K. Kinoshita, F. Matsukura, N. Kasai, and H. Ohno, 2014, "Comprehensive study of $\mathrm{CoFeB}-\mathrm{MgO}$ magnetic tunnel junction characteristics with single- and double-interface scaling down to $1 \mathrm{X} \mathrm{nm}$," Electron Devices Meeting (IEDM), 2013 IEEE International, pp. 33.2.1-33.2.4.

Sato, H., M. Yamanouchi, K. Miura, S. Ikeda, H. D. Gan, K. Mizunuma, R. Koizumi, F. Matsukura, and H. Ohno, 2011, "Junction size effect on switching current and thermal stability in $\mathrm{CoFeB} / \mathrm{MgO}$ perpendicular magnetic tunnel junctions," Appl. Phys. Lett. 99, 042501.

Savtchenko, L., B. Engels, N. Rizzo, M. DeHerrera, and J. A. Janesky, 2003, "Method of writing to scalable magnetoresistance random access memory element," Patent Application US $6,545,906$.

Sbiaa, R., H. Meng, and S. N. Piramanayagam, 2011, "Materials with perpendicular magnetic anisotropy for magnetic random access memory," Phys. Status Solidi RRL 5, 413-419.

Scheck, C., L. Cheng, I. Barsukov, Z. Frait, and W. E. Bailey, 2007, "Low relaxation rate in epitaxial vanadium-doped ultrathin iron films," Phys. Rev. Lett. 98, 117601.

Schellekens, A. J., A. van den Brink, J. H. Franken, H. J. M. Swagten, and B. Koopmans, 2012, "Electric-field control of domain wall motion in perpendicularly magnetized materials," Nat. Commun. 3, 847.

Seki, T., M. Kohda, J. Nitta, and K. Takanashi, 2011, "Coercivity change in an FePt thin layer in a Hall device by voltage application," Appl. Phys. Lett. 98, 212505.

Shaw, J. M., H. T. Nembach, and T. J. Silva, 2012, "Determination of spin pumping as a source of linewidth in sputtered $\mathrm{Co}_{90} \mathrm{Fe}_{10} / \mathrm{Pd}$ multilayers by use of broadband ferromagnetic resonance spectroscopy," Phys. Rev. B 85, 054412.

Shaw, J. M., H. T. Nembach, M. Weiler, T. J. Silva, M. Schoen, J. Z. Sun, and D.C. Worledge, 2015, "Perpendicular Magnetic Anisotropy and Easy Cone State in $\mathrm{Ta} / \mathrm{Co}_{60} \mathrm{Fe}_{20} \mathrm{~B}_{20} / \mathrm{MgO}$," IEEE Magn. Lett. 6, 3500404.

Shimabukuro, R., K. Nakamura, T. Akiyama, and T. Ito, 2010, "Electric field effects on magnetocrystalline anisotropy in ferromagnetic Fe monolayers," Physica E: Low-dimens. Syst. Nanostruct. 42, 1014-1017.

Shiota, Y., T. Maruyama, T. Nozaki, T. Shinjo, M. Shiraishi, and Y. Suzuki, 2009, "Voltage-assisted magnetization switching in ultrathin $\mathrm{Fe}_{80} \mathrm{Co}_{20}$ alloy layers," Appl. Phys. Express 2, 063001.

Shiota, Y., T. Nozaki, F. Bonell, S. Murakami, T. Shinjo, and Y. Suzuki, 2012, "Induction of coherent magnetization switching in a few atomic layers of FeCo using voltage pulses," Nat. Mater. 11, 39-43.

Sicot, M., S. Andrieu, F. Bertran, and F. Fortuna, 2005, "Electronic properties of $\mathrm{Fe}, \mathrm{Co}$, and $\mathrm{Mn}$ ultrathin films at the interface with MgO(001)," Phys. Rev. B 72, 144414.

Sinha, J., et al., 2013, "Enhanced interface perpendicular magnetic anisotropy in $\mathrm{Ta} / \mathrm{CoFeB} / \mathrm{MgO}$ using nitrogen doped Ta underlayers," Appl. Phys. Lett. 102, 242405.

Skomski, R., 1998, "Magnetoelectric Néel anisotropies," IEEE Trans. Magn. 34, 1207-1209.

Skomski, R., A. Kashyap, A. Solanki, A. Enders, and D. J. Sellmyer, 2010, "Magnetic anisotropy in itinerant magnets," J. Appl. Phys. 107, 09A735.

Slonczewski, J. C., 1996, "Current driven excitations of magnetic multilayers,” J. Magn. Magn. Mater. 159, L1-L7.

Slonczewski, J. C., 1999, "Excitation of spin waves by an electric current,” J. Magn. Magn. Mater. 195, L261-L268. 
Sokalski, V., M. T. Moneck, E. Yang, and J. G. Zhu, 2012, "Optimization of Ta thickness for perpendicular magnetic tunnel junction applications in the MgO-FeCoB-Ta system," Appl. Phys. Lett. 101, 072411.

Stainer, Q., L. Lombard, K. Mackay, D. Lee, S. Bandiera, C. Portemont, C. Creuzet, R. C. Sousa, and B. Dieny, 2014, "Self-referenced multi-bit thermally assisted magnetic random access memories," Appl. Phys. Lett. 105, 032405.

Stipe, B., et al., 2010, "Magnetic recording at $1.5 \mathrm{~Pb} / \mathrm{m}^{2}$ using an integrated plasmonic antenna," Nat. Photonics 4, 484-488.

Suess, D., T. Schrefl, S. Fähler, M. Kirschner, G. Hrkac, F. Dorfbauer, and J. Fidler, 2005, "Exchange spring media for perpendicular recording," Appl. Phys. Lett. 87, 012504.

Sugita, Y., Y. Kawawake, M. Satomi, and H. Sakakima, 2001, "Thermal stability of PtMn based synthetic spin valves using thin oxide layer," J. Appl. Phys. 89, 6919-6921.

Sun, J.Z., 2000, "Spin-current interaction with a monodomain magnetic body: A model study," Phys. Rev. B 62, 570-578.

Sun, J.Z., 2006, "Spin angular momentum transfer in currentperpendicular nanomagnetic junctions," IBM J. Res. Dev. 50, 81-100.

Suzuki, S., S. Yasuda, K. Edakawa, and S. Seki, 2013, "Firstprinciples study of electric field effects on magnetic anisotropy in $\mathrm{MgO} / \mathrm{TM} / \mathrm{Au}(\mathrm{TM}=\mathrm{Fe}, \mathrm{Co})$ systems," J. Phys. Soc. Jpn. 82, 124715.

Suzuki, T., et al., 2011, "Current-induced effective field in perpendicularly magnetized Ta/CoFeB/MgO wire," Appl. Phys. Lett. 98, 142505 .

Takayama, H., K.-P. Bohnen, and P. Fulde, 1976, "Magnetic surface anisotropy of transition metals," Phys. Rev. B 14, 2287-2295.

Tartaj, P., M.P. Morales, T. González-Carreño, S. VeintemillasVerdaguer, and C. J. Serna, 2005, "Advances in magnetic nanoparticles for biotechnology applications,” J. Magn. Magn. Mater. 290-291, 28-34.

Thiaville, A., S. Rohart, E. Jué, V. Cros, and A. Fert, 2012, "Dynamics of Dzyaloshinskii domain walls in ultrathin magnetic films," Europhys. Lett. 100, 57002.

Thomas, L., et al., 2014, "Perpendicular spin transfer torque magnetic random access memories with high spin torque efficiency and thermal stability for embedded applications," J. Appl. Phys. 115, 172615.

Thompson, D. A., and J. S. Best, 2000, “The future of magnetic data storage technology," IBM J. Res. Dev. 44, 311-332.

Timopheev, A., R. Sousa, M. Chshiev, H. T. Nguyen, and B. Dieny, 2016, "Second order anisotropy contribution in perpendicular magnetic tunnel junctions," Sci. Rep. 6, 26877.

Tomita, H., et al., 2013, "Unified understanding of both thermally assisted and precessional spin-transfer switching in perpendicularly magnetized giant magnetoresistive nanopillars," Appl. Phys. Lett. 102, 042409.

Tsai, C. C., C. W. Cheng, M. C. Tsai, and G. Chern, 2014, "Superparamagnetic states and perpendicular magnetic anisotropy in ultrathin $\mathrm{MgO} / \mathrm{CoFeB} / \mathrm{Ta}$ structures," IEEE Trans. Magn. 50, 1401404.

Tsai, W. C., S. C. Liao, H. C. Hou, C. T. Yen, Y. H. Wang, H. M. Tsai, F. H. Chang, H. J. Lin, and C. H. Lai, 2012, "Investigation of perpendicular magnetic anisotropy of $\mathrm{CoFeB}$ by X-ray magnetic circular dichroism," Appl. Phys. Lett. 100, 172414.

Tserkovnyak, Y., A. Brataas, and G.E.W. Bauer, 2002, "Spin pumping and magnetization dynamics in metallic multilayers," Phys. Rev. B 66, 224403.
Tsoi, M., A. G. M. Jansen, J. Bass, W.-C. Chiang, M. Seck, V. Tsoi, and P. Wyder, 1998, "Excitation of a magnetic multilayer by an electric current," Phys. Rev. Lett. 80, 4281-4284.

Tsujikawa, M., S. Haraguchi, T. Oda, Y. Miura, and M. Shirai, 2011, "A comparative $a b$ initio study on electric-field dependence of magnetic anisotropy in $\mathrm{MgO} / \mathrm{Fe} / \mathrm{Pt}$ and $\mathrm{MgO} / \mathrm{Fe} / \mathrm{Au}$ films,' J. Appl. Phys. 109, $07 \mathrm{C} 107$.

Tsujikawa, M., and T. Oda, 2009, "Finite electric field effects in the large perpendicular magnetic anisotropy surface $\mathrm{Pt} / \mathrm{Fe} / \mathrm{Pt}(001)$ : A first-principles study," Phys. Rev. Lett. 102, 247203.

Tsunegi, S., H. Kubota, S. Tamaru, K. Yakushiji, M. Konoto, A. Fukushima, T. Tanigushi, H. Arai, H. Imamura, and S. Yuasa, 2014, "Damping parameter and interfacial perpendicular magnetic anisotropy of $\mathrm{FeB}$ nanopillar sadwhiched between $\mathrm{MgO}$ barrier and cap layers in magnetic tunnel junctions," Appl. Phys. Express 7, 033004.

Újfalussy, B., L. Szunyogh, P. Bruno, and P. Weinberger, 1996, "First-principles calculation of the anomalous perpendicular anisotropy in a Co monolayer on Au(111)," Phys. Rev. Lett. 77, 1805-1808.

Vadapoo, R., A. Hallal, H. X. Yang, and M. Chshiev, 2016, "Firstprinciples investigation of magnetocrystalline anisotropy at the L21 full HeuslerlMgO inerfaces and tunnel junctions," Phys. Rev. B 94, 104418 .

van der Laan, G, 1998, "Microscopic origin of magnetocrystalline anisotropy in transition metal thin films," J. Phys. Condens. Matter 10, 3239 .

Varga, L., and W. D. Doyle, 1996, "Measurement of the crystalline anisotropy in sputtered single crystal FeTaN thin films," J. Appl. Phys. 79, 4995-4997.

Vedyayev, A., N. Strelkov, M. Chshiev, N. Ryzhanova, and B. Dieny, 2011, "Spin transfer torques induced by spin Hall effect," arXiv:1108.2589v1.

Veloso, A., P. P. Freitas, P. Wei, N. P. Barradas, J. C. Soares, B. Almeida, and J. B. Sousa, 2000, "Magnetoresistance enhancement in specular, bottom-pinned, $\mathrm{Mn}_{83} \mathrm{Ir}_{17}$ spin valves with nano-oxide layers," Appl. Phys. Lett. 77, 1020-1022.

Vo-Van, C., et al., 2010, "Ultrathin epitaxial cobalt films on graphene for spintronic investigations and applications," New J. Phys. 12, 103040.

Wang, C. L., S. H. Huang, C. H. Lai, W. C. Chen, S. Y. Yang, K. H. Shen, and H. Y. Bor, 2009, "Reduction in critical current density by tuning damping constants of $\mathrm{CoFeB}$ for spin torque transfer switching," J. Phys. D 42, 115006.

Wang, D.-S., R. Wu, and A. J. Freeman, 1993a, "Magnetocrystalline anisotropy of Co-Pd interfaces," Phys. Rev. B 48, 15886-15892.

Wang, D.-S., R. Wu, and A. J. Freeman, 1993b, "First-principles theory of surface magnetocrystalline anisotropy and the diatomicpair model," Phys. Rev. B 47, 14932-14947.

Wang, W-G., M. Li, S. Hageman, and C. L. Chien, 2012, "Electricfield-assisted switching in magnetic tunnel junctions," Nat. Mater. 11, 64-68.

Wang, K.-L., H. Lee, and P. K. Amiri, 2015, "Magnetoelectric Random Access Memory-Based Circuit Design by Using Voltage-Controlled Magnetic Anisotropy in Magnetic Tunnel Junctions," IEEE Trans. Nanotechnol. 14, 992.

Wang, X., and A. Manchon, 2012, "Diffusive spin dynamics in ferromagnetic thin films with a Rashba interaction," Phys. Rev. Lett. 108, 117201.

Wang, X.D., D. S. Wang, R. Q. Wu, and A. J. Freeman, 1996, "Validity of the force theorem for magnetocrystalline anisotropy," J. Magn. Magn. Mater. 159, 337-341. 
Wegrowe, J.E., D. Kelly, Y. Jaccard, P. Guittienne, and J.-P. Ansermet, 1999, "Current-induced magnetization reversal in magnetic nanowires," Europhys. Lett. 45, 626-632.

Weinert, M., R. E. Watson, and J. W. Davenport, 1985, “Total-energy differences and eigenvalue sums," Phys. Rev. B 32, 2115-2119.

Weisheit, M., S. Fähler, A. Marty, Y. Souche, C. Poinsignon, and D. Givord, 2007, "Electric field-induced modification of magnetism in thin-film ferromagnets," Science 315, 349-351.

Wolf, S. A., J. Lu, M. R. Stan, E. Chen, and D. M. Treger, 2010, “The promise of nanomagnetics and spintronics for future logic and universal memory," Proc. IEEE 98, 2155-2168.

Worledge, D. C., 2010, "Seed layer and free magnetic layer for perpendicular anisotropy in a spin-torque magnetic random access memory," Patent Application US 0,303,995A1.

Worledge, D. C., G. Hu, D. Abraham, J.Z. Sun, P. L. Trouilloud, J. Nowak, S. Brown, M. C. Gaidis, E. J. O'Sullivan, and R. P. Robertazzi, 2011, "Spin torque switching of perpendicular $\mathrm{Ta} /$ $\mathrm{CoFeB} / \mathrm{MgO}$-based magnetic tunnel junctions," Appl. Phys. Lett. 98, 022501 .

Worledge, D. C., G. Hu, D. W. Abraham, P. L. Trouilloud, and S. Brown, 2014, "Development of perpendicularly magnetized $\mathrm{Ta} / \mathrm{CoFeB} / \mathrm{MgO}$-based tunnel junctions at IBM," J. Appl. Phys. 115, 172601.

Worledge, D. C., and P.L. Trouilloud, 2003, "Magnetoresistance measurement of unpatterned magnetic tunnel junction wafers by current-in-plane tunnelling," Appl. Phys. Lett. 83, 84-86.

$\mathrm{Wu}$, R., and A.J. Freeman, 1999, "Spin-orbit induced magnetic phenomena in bulk metals and their surfaces and interfaces," J. Magn. Magn. Mater. 200, 498-514.

Yakata, S., H. Kubota, Y. Suzuki, K. Yakushiji, A. Fukushima, S. Yuasa, and K. Ando, 2009, "Influence of perpendicular magnetic anisotropy on spin transfer switching current in $\mathrm{CoFeB} /$ $\mathrm{MgO} / \mathrm{CoFeB}$ magnetic tunnel junctions," J. Appl. Phys. 105, 07D131.

Yakushiji, K., T. Saruya, H. Kubota, A. Fukushima, T. Nagahama, S. Yuasa, and K. Ando, 2010, "Ultrathin $\mathrm{Co} / \mathrm{Pt}$ and $\mathrm{Co} / \mathrm{Pd}$ superlattice films for $\mathrm{MgO}$-based perpendicular magnetic tunnel junctions," Appl. Phys. Lett. 97, 232508.

Yamanouchi, M., L. Chen, J. Kim, M. Hayashi, H. Sato, S. Fukami, S. Ikeda, F. Matsukura, and H. Ohno, 2013, "Three terminal magnetic tunnel junction utilizing the spin Hall effect of iridiumdoped copper," Appl. Phys. Lett. 102, 212408.

Yang, H. X., 2012, "First-principles study of spintronic phenomena in magnetic tunnel junctions and graphene," Ph.D. thesis, University of Grenoble.

Yang, H. X., M. Chshiev, B. Dieny, J. H. Lee, A. Manchon, and K. H. Shin, 2011, "First principles investigation of the very large perpendicular magnetic anisotropy at $\mathrm{Fe} \mid \mathrm{MgO}$ and $\mathrm{Co} \mid \mathrm{MgO}$ interfaces," Phys. Rev. B 84, 054401.

Yang, H. X., A. Thiaville, S. Rohart, A. Fert, and M. Chshiev, 2015, "Anatomy of Dzyaloshinskii-Moriya Interaction at Co/Pt Interfaces,” Phys. Rev. Lett. 115, 267210.

Yang, H.X., A. D. Vu, A. Hallal, N. Rougemaille, J. Coraux, G. Chen, A. K. Schmid, and M. Chshiev, 2016, "Anatomy and Giant Enhancement of the Perpendicular Magnetic Anisotropy of CobaltGraphene Heterostructures," Nano Lett. 16, 145.

Yang, I., S. Y. Savrasov, and G. Kotliar, 2001, "Importance of Correlation Effects on Magnetic Anisotropy in Fe and $\mathrm{Ni}$," Phys. Rev. Lett. 87, 216405.
Yang, S., J. Lee, G. An, J. Kim, W. Chung, and J. P. Hong, 2014, "Thermally stable perpendicular magnetic anisotropy features of $\mathrm{Ta} / \mathrm{TaOx} / \mathrm{Ta} / \mathrm{CoFeB} / \mathrm{MgO} / \mathrm{W}$ stacks via $\mathrm{TaOx}$ underlayer insertion," J. Appl. Phys. 116, 113902.

Yoda, H., S. Fujita, N. Shimomura, E. Kitagawa, K. Abe, K. Nomura, H. Noguchi, and J. Ito, 2012, "Progress of STT-MRAM technology and the effect on normally-off computing systems," Electron Devices Meeting (IEDM), 2012 IEEE International, 11.3.1-11.3.4.

Yoda, H., et al., 2010, "High efficient spin transfer torque writing on perpendicular magnetic tunnel junctions for high density MRAMs," Curr. Appl. Phys. 10, e87-e90.

You, C. Y., T. Ohkubo, Y. K. Takahashi, and K. Hono, 2008, "Boron segregation in crystallized $\mathrm{MgO} /$ amorphous $\mathrm{Co}_{40} \mathrm{Fe}_{40} \mathrm{~B}_{20}$ thin films," J. Appl. Phys. 104, 033517.

Yu, G., et al., 2014, "Switching of perpendicular magnetization by spin-orbit torques in the absence of external magnetic fields," Nat. Nanotechnol. 9, 548-554.

Yu, G., et al., 2015, "Strain-induced modulation of perpendicular magnetic anisotropy in $\mathrm{Ta} / \mathrm{CoFeB} / \mathrm{MgO}$ structures investigated by ferromagnetic resonance," Appl. Phys. Lett. 106, 072402 .

Yu, X. Z., Y. Onose, N. Kanazawa, J. H. Park, J. H. Han, Y. Matsui, N. Nagaosa, and Y. Tokura, 2010, "Real-space observation of a two-dimensional skyrmion crystal," Nature (London) $\mathbf{4 6 5}$, 901-904.

Yuasa, S., and D. D. Djayaprawira, 2007, "Giant tunnel magnetoresistance in magnetic tunnel junctions with a crystalline $\mathrm{MgO}$ (001) barrier," J. Phys. D 40, R337-R354.

Yuasa, S., A. Fukushima, H. Kubota, Y. Suzuki, and K. Ando, 2006, "Giant tunneling magnetoresistance up to $410 \%$ at room temperature in fully epitaxial $\mathrm{Co} / \mathrm{MgO} / \mathrm{Co}$ magnetic tunnel junctions with bcc Co(001) electrodes," Appl. Phys. Lett. 89, 042505.

Yuasa, S., T. Nagahama, A. Fukushima, Y. Suzuki, and K. Ando, 2004, "Giant room temperature magnetoresistance in single-crystal $\mathrm{Fe} / \mathrm{MgO} / \mathrm{Fe}$ magnetic tunnel junctions," Nat. Mater. 3, 868-871.

Yuasa, S., Y. Suzuki, T. Katayama, and K. Ando, 2005, "Characterization of growth and crystallization processes in $\mathrm{CoFeB} / \mathrm{MgO}$ / $\mathrm{CoFeB}$ magnetic tunnel junction structure by reflective high energy electron diffraction," Appl. Phys. Lett. 87, 242503.

Zhang, J., C. Franz, M. Czerner, and C. Heiliger, 2014, "Perpendicular magnetic anisotropy in $\mathrm{CoFe} / \mathrm{MgO} / \mathrm{CoFe}$ magnetic tunnel junctions by first-principles calculations," Phys. Rev. B 90, 184409.

Zhang, S., and Z. Li, 2004, "Roles of non-equilibrium conduction electrons on the magnetization dynamics of ferromagnets," Phys. Rev. Lett. 93, 127204.

Zhang, X.-G., W. H. Butler, and A. Bandyopadhyay, 2003, "Effects of the iron-oxide layer in $\mathrm{Fe}-\mathrm{FeO}-\mathrm{MgO}-\mathrm{Fe}$ tunneling junctions," Phys. Rev. B 68, 092402.

Zhu, J., et al., 2012, "Voltage-Induced Ferromagnetic Resonance in Magnetic Tunnel Junctions," Phys. Rev. Lett. 108, 197203.

Zhu, J. G., X. Zhu, and Y. Tang, 2008, "Microwave-assisted magnetic recording," IEEE Trans. Magn. 44, 125-131.

Zhu, W., D. Xiao, Y. Liu, S. J. Gong, and C.-G. Duan, 2014, "Picosecond electric field pulse induced coherent magnetic switching in $\mathrm{MgO} / \mathrm{FePt} / \mathrm{Pt}(001)$-based tunnel junctions: A multiscale study," Sci. Rep. 4, 4117. 